\title{
Can quantum probability provide a new direction for cognitive modeling?
}

\author{
Emmanuel M. Pothos \\ Department of Psychology, City University London, London EC1V OHB, \\ United Kingdom \\ emmanuel.pothos.1@ city.ac.uk \\ http://www.staff.city.ac.uk/ sbbh932/ \\ Jerome R. Busemeyer \\ Department of Psychological and Brain Sciences, Indiana University, \\ Bloomington, IN 47405 \\ jbusemey@indiana.edu \\ http://mypage.iu.edu/ jbusemey/home.html
}

\begin{abstract}
Classical (Bayesian) probability (CP) theory has led to an influential research tradition for modeling cognitive processes. Cognitive scientists have been trained to work with CP principles for so long that it is hard even to imagine alternative ways to formalize probabilities. However, in physics, quantum probability (QP) theory has been the dominant probabilistic approach for nearly 100 years. Could QP theory provide us with any advantages in cognitive modeling as well? Note first that both CP and QP theory share the fundamental assumption that it is possible to model cognition on the basis of formal, probabilistic principles. But why consider a QP approach? The answers are that (1) there are many well-established empirical findings (e.g., from the influential Tversky, Kahneman research tradition) that are hard to reconcile with CP principles; and (2) these same findings have natural and straightforward explanations with quantum principles. In QP theory, probabilistic assessment is often strongly context- and orderdependent, individual states can be superposition states (that are impossible to associate with specific values), and composite systems can be entangled (they cannot be decomposed into their subsystems). All these characteristics appear perplexing from a classical perspective. However, our thesis is that they provide a more accurate and powerful account of certain cognitive processes. We first introduce QP theory and illustrate its application with psychological examples. We then review empirical findings that motivate the use of quantum theory in cognitive theory, but also discuss ways in which QP and CP theories converge. Finally, we consider the implications of a QP theory approach to cognition for human rationality.
\end{abstract}

Keywords: category membership; classical probability theory; conjunction effect; decision making; disjunction effect; interference effects; judgment; quantum probability theory; rationality; similarity ratings

\section{Preliminary issues}

\subsection{Why move toward quantum probability theory?}

In this article we evaluate the potential of quantum probability (QP) theory for modeling cognitive processes. What is the motivation for employing QP theory in cognitive modeling? Does the use of QP theory offer the promise of any unique insights or predictions regarding cognition? Also, what do quantum models imply regarding the nature of human rationality? In other words, is there anything to be gained, by seeking to develop cognitive models based on QP theory? Especially over the last decade, there has been growing interest in such models, encompassing publications in major journals, special issues, dedicated workshops, and a comprehensive book (Busemeyer \& Bruza 2012). Our strategy in this article is to briefly introduce QP theory, summarize progress with selected, QP models, and motivate answers to the abovementioned questions. We note that this article is not about the application of quantum physics to brain physiology. This is a controversial issue (Hammeroff 2007; Litt et al. 2006) about which we are agnostic. Rather, we are interested in QP theory as a mathematical framework for cognitive modeling. QP theory is potentially relevant in any behavioral situation that involves uncertainty. For example, Moore (2002) reported that the likelihood of a "yes" response to the questions "Is Gore honest?" and "Is Clinton honest?” depends on the relative order of the questions. We will subsequently discuss how QP principles can provide a simple and intuitive account for this and a range of other findings.

QP theory is a formal framework for assigning probabilities to events (Hughes 1989; Isham 1989). QP theory can be distinguished from quantum mechanics, the latter being a theory of physical phenomena. For the present purposes, it is sufficient to consider QP theory as the abstract foundation of quantum mechanics not specifically tied to physics (for more refined characterizations see, e.g., Aerts \& Gabora 2005b; Atmanspacher et al. 2002; Khrennikov 2010; Redei \& Summers 2007). The development of quantum theory has been the result of intense effort from some of the greatest scientists of all time, over a period of $>30$ years. The idea of "quantum" was first proposed by Planck in the early 1900s and advanced by Einstein. Contributions from Bohr, Born, Heisenberg, and Schrödinger all led to the eventual formalization of $\mathrm{QP}$ 
Pothos \& Busemeyer: Can quantum probability provide a new direction for cognitive modeling?

theory by von Neumann and Dirac in the 1930s. Part of the appeal of using QP theory in cognition relates to confidence in the robustness of its mathematics. Few other theoretical frameworks in any science have been scrutinized so intensely, led to such surprising predictions, and, also, changed human existence as much as QP theory (when applied to the physical world; quantum mechanics has enabled the development of, e.g., the transistor, and, therefore, the microchip and the laser).

QP theory is, in principle, applicable not just in physics, but in any science in which there is a need to formalize uncertainty. For example, researchers have been pursuing applications in areas as diverse as economics (Baaquie 2004) and information theory (e.g., Grover 1997; Nielsen \& Chuang 2000). The idea of using quantum theory in psychology has existed for nearly 100 years: Bohr, one of the founding fathers of quantum theory, was known to believe that aspects of quantum theory could provide insight about cognitive process (Wang et al., in press). However, Bohr never made any attempt to provide a formal cognitive model based on QP theory, and such models have started appearing only fairly recently (Aerts

Emmanuel Pothos studied physics at Imperial College, during which time he obtained the Stanley Raimes Memorial prize in mathematics, and continued with a doctorate in experimental psychology at Oxford University. He has worked with a range of computational frameworks for cognitive modeling, including ones based on information theory, flexible representation spaces, Bayesian methods, and, more recently, quantum theory. He has authored approximately sixty journal articles on related topics, as well as on applications of cognitive methods to health and clinical psychology. Pothos is currently a senior lecturer in psychology at City University London.

Jerome Busemeyer received his PhD as a mathematical psychologist from University of South Carolina in 1980, and later he enjoyed a post-doctoral position at University of Illinois. For 14 years he was a faculty member at Purdue University. He moved on to Indiana University, where he is provost professor, in 1997. Busemeyer's research has been steadily funded by the National Science Foundation, National Institute of Mental Health, and National Institute on Drug Abuse, and in return he served on national grant review panels for these agencies. He has published over 100 articles in various cognitive and decision science journals, such as Psychological Review, as well as serving on their editorial boards. He served as chief editor of Journal of Mathematical Psychology from 2005 through 2010 and he is currently an associate editor of Psychological Review. From 2005 through 2007, Busemeyer served as the manager of the Cognition and Decision Program at the Air Force Office of Scientific Research. He became a fellow of the Society of Experimental Psychologists in 2006. His research includes mathematical models of learning and decision making, and he formulated a dynamic theory of human decision making called decision field theory. Currently, he is working on a new theory applying quantum probability to human judgment and decision making, and he published a new book on this topic with Cambridge University Press.
\& Aerts 1995; Aerts \& Gabora 2005b; Atmanspacher et al. 2004; Blutner 2009; Bordley 1998; Bruza et al. 2009; Busemeyer et al. 2006b; Busemeyer et al. 2011; Conte et al. 2009; Khrennikov 2010; Lambert-Mogiliansky et al. 2009; Pothos \& Busemeyer 2009; Yukalov \& Sornette 2010). But what are the features of quantum theory that make it a promising framework for understanding cognition? It seems essential to address this question before expecting readers to invest the time for understanding the (relatively) new mathematics of QP theory.

Superposition, entanglement, incompatibility, and interference are all related aspects of QP theory, which endow it with a unique character. Consider a cognitive system, which concerns the cognitive representation of some information about the world (e.g., the story about the hypothetical Linda, used in Tversky and Kahneman's [1983] famous experiment; sect. 3.1 in this article). Questions posed to such systems ("Is Linda feminist?") can have different outcomes (e.g., "Yes, Linda is feminist"). Superposition has to do with the nature of uncertainty about question outcomes. The classical notion of uncertainty concerns our lack of knowledge about the state of the system that determines question outcomes. In QP theory, there is a deeper notion of uncertainty that arises when a cognitive system is in a superposition among different possible outcomes. Such a state is not consistent with any single possible outcome (that this is the case is not obvious; this remarkable property follows from the Kochen-Specker theorem). Rather, there is a potentiality (Isham 1989, p. 153) for different possible outcomes, and if the cognitive system evolves in time, so does the potentiality for each possibility. In quantum physics, superposition appears puzzling: what does it mean for a particle to have a potentiality for different positions, without it actually existing at any particular position? By contrast, in psychology, superposition appears an intuitive way to characterize the fuzziness (the conflict, ambiguity, and ambivalence) of everyday thought.

Entanglement concerns the compositionality of complex cognitive systems. QP theory allows the specification of entangled systems for which it is not possible to specify a joint probability distribution from the probability distributions of the constituent parts. In other words, in entangled composite systems, a change in one constituent part of the system necessitates changes in another part. This can lead to interdependencies among the constituent parts not possible in classical theory, and surprising predictions, especially when the parts are spatially or temporally separated.

In quantum theory, there is a fundamental distinction between compatible and incompatible questions for a cognitive system. Note that the terms compatible and incompatible have a specific, technical meaning in QP theory, which should not be confused with their lay use in language. If two questions, $\mathrm{A}$ and $\mathrm{B}$, about a system are compatible, it is always possible to define the conjunction between A and B. In classical systems, it is assumed by default that all questions are compatible. Therefore, for example, the conjunctive question "are A and B true" always has a yes or no answer and the order between questions $\mathrm{A}$ and $\mathrm{B}$ in the conjunction does not matter. By contrast, in QP theory, if two questions $\mathrm{A}$ and $\mathrm{B}$ are incompatible, it is impossible to define a single question regarding their conjunction. This is because an answer to question A implies a superposition state regarding question $\mathrm{B}$ (e.g., if $\mathrm{A}$ is true at a time point, then $\mathrm{B}$ can be neither true nor false at the 
same time point). Instead, QP defines conjunction between incompatible questions in a sequential way, such as "A and then B." Crucially, the outcome of question A can affect the consideration of question B, so that interference and order effects can arise. This is a novel way to think of probability, and one that is key to some of the most puzzling predictions of quantum physics. For example, knowledge of the position of a particle imposes uncertainty on its momentum. However, incompatibility may make more sense when considering cognitive systems and, in fact, it was first introduced in psychology. The physicist Niels Bohr borrowed the notion of incompatibility from the work of William James. For example, answering one attitude question can interfere with answers to subsequent questions (if they are incompatible), so that their relative order becomes important. Human judgment and preference often display order and context effects, and we shall argue that in such cases quantum theory provides a natural explanation of cognitive process.

\subsection{Why move away from existing formalisms?}

By now, we hope we have convinced readers that QP theory has certain unique properties, whose potential for cognitive modeling appears, at the very least, intriguing. For many researchers, the inspiration for applying quantum theory in cognitive modeling has been the widespread interest in cognitive models based on CP theory (Anderson 1991; Griffiths et al. 2010; Oaksford \& Chater 2007; Tenenbaum et al. 2011). Both CP and QP theories are formal probabilistic frameworks. They are founded on different axioms (the Kolmogorov and Dirac/von Neumann axioms, respectively) and, therefore, often produce divergent predictions regarding the assignment of probabilities to events. However, they share profound commonalities as well, such as the central objective of quantifying uncertainty, and similar mechanisms for manipulating probabilities. Regarding cognitive modeling, quantum and classical theorists share the fundamental assumption that human cognition is best understood within a formal probabilistic framework.

As Griffiths et al. (2010, p. 357) note, "probabilistic models of cognition pursue a top-down or 'function-first' strategy, beginning with abstract principles that allow agents to solve problems posed by the world ... and then attempting to reduce these principles to psychological and neural processes." That is, the application of CP theory to cognition requires a scientist to create hypotheses regarding cognitive representations and inductive biases and, therefore, elucidate the fundamental questions of how and why a cognitive problem is successfully addressed. In terms of Marr's (1982) analysis, CP models are typically aimed at the computational and algorithmic levels, although perhaps it is more accurate to characterize them as top down or function first (as Griffiths et al. 2010, p. 357).

We can recognize the advantage of CP cognitive models in at least two ways. First, in a CP cognitive model, the principles that are invoked (the axioms of CP theory) work as a logical "team" and always deductively constrain each other. By contrast, alternative cognitive modeling approaches (e.g., based on heuristics) work "alone" and therefore are more likely to fall foul of arbitrariness problems, whereby it is possible to manipulate each principle in the model independently of other principles. Second, neuroscience methods and computational bottom-up approaches are typically unable to provide much insight into the fundamental why and how questions of cognitive process (Griffiths et al. 2010). Overall, there are compelling reasons for seeking to understand the mind with CP theory. The intention of QP cognitive models is aligned with that of $\mathrm{CP}$ models. Therefore, it makes sense to present QP theory side by side with CP theory, so that readers can appreciate their commonalities and differences.

A related key issue is this: if CP theory is so successful and elegant (at least, in cognitive applications), why seek an alternative? Moreover, part of the motivation for using $\mathrm{CP}$ theory in cognitive modeling is the strong intuition supporting many CP principles. For example, the probability of $A$ and $B$ is the same as the probability of $B$ and $A$ $(\operatorname{Prob}(\mathrm{A} \& \mathrm{~B})=\operatorname{Prob}(\mathrm{A} \& \mathrm{~B}))$. How can it be possible that the probability of a conjunction depends upon the order of the constituents? Indeed, as Laplace (1816, cited in Perfors et al. 2011) said, "probability theory is nothing but common sense reduced to calculation." By contrast, QP theory is a paradigm notorious for its conceptual difficulties (in the 1960s, Feynman famously said "I think I can safely say that nobody understands quantum mechanics"). A classical theorist might argue that, when it comes to modeling psychological intuition, we should seek to apply a computational framework that is as intuitive as possible (CP theory) and avoid the one that can lead to puzzling and, superficially at least, counterintuitive predictions (QP theory).

Human judgment, however, often goes directly against CP principles. A large body of evidence has accumulated to this effect, mostly associated with the influential research program of Tversky and Kahneman (Kahneman et al. 1982; Tversky \& Kahneman 1973; 1974; Tversky \& Shafir 1992). Many of these findings relate to order/context effects, violations of the law of total probability (which is fundamental to Bayesian modeling), and failures of compositionality. Therefore, if we are to understand the intuition behind human judgment in such situations, we have to look for an alternative probabilistic framework. Quantum theory was originally developed so as to model analogous effects in the physical world and therefore, perhaps, it can offer insight into those aspects of human judgment that seem paradoxical from a classical perspective. This situation is entirely analogous to that faced by physicists early in the last century. On the one hand, there was the strong intuition from classical models (e.g., Newtonian physics, classical electromagnetism). On the other hand, there were compelling empirical findings that were resisting explanation on the basis of classical formalisms. Therefore, physicists had to turn to quantum theory, and so paved the way for some of the most impressive scientific achievements.

It is important to note that other cognitive theories embody order/context effects or interference effects or other quantum-like components. For example, a central aspect of the gestalt theory of perception concerns how the dynamic relationships among the parts of a distal layout together determine the conscious experience corresponding to the image. Query theory (Johnson et al. 2007) is a proposal for how value is constructed through a series of (internal) queries, and has been used to explain the endowment effect in economic choice. In query theory, value is constructed, rather than read off, and also different 
Pothos \& Busemeyer: Can quantum probability provide a new direction for cognitive modeling?

queries can interfere with each other, so that query order matters. In configural weight models (e.g., Birnbaum 2008) we also encounter the idea that, in evaluating gambles, the context of a particular probability-consequence branch (e.g., its rank order) will affect its weight. The theory also allows weight changes depending upon the observer perspective (e.g., buyer vs. seller). Anderson's (1971) integration theory is a family of models for how a person integrates information from several sources, and also incorporates a dependence on order. Fuzzy trace theory (Reyna 2008; Reyna \& Brainerd 1995) is based on a distinction between verbatim and gist information, the latter corresponding to the general semantic qualities of an event. Gist information can be strongly context and observer dependent and this has led fuzzy trace theory to some surprising predictions (e.g., Brainerd et al. 2008).

This brief overview shows that there is a diverse range of cognitive models that include a role for context or order, and a comprehensive comparison is not practical here. However, when comparisons have been made, the results favored quantum theory (e.g., averaging theory was shown to be inferior to a matched quantum model, Trueblood \& Busemeyer 2011). In some other cases, we can view QP theory as a way to formalize previously informal conceptualizations (e.g., for query theory and the fuzzy trace theory).

Overall, there is a fair degree of flexibility in the particular specification of computational frameworks in cognitive modeling. In the case of CP and QP models, this flexibility is tempered by the requirement of adherence to the axioms in each theory: all specific models have to be consistent with these axioms. This is exactly what makes CP (and QP) models appealing to many theorists and why, as noted, in seeking to understand the unique features of QP theory, it is most natural to compare it with CP theory.

In sum, a central aspect of this article is the debate about whether psychologists should explore the utility of quantum theory in cognitive theory; or whether the existing formalisms are (mostly) adequate and a different paradigm is not necessary. Note that we do not develop an argument that CP theory is unsuitable for cognitive modeling; it clearly is, in many cases. And, moreover, as will be discussed, CP and QP processes sometimes converge in their predictions. Rather, what is at stake is whether there are situations in which the distinctive features of QP theory provide a more accurate and elegant explanation for empirical data. In the next section we provide a brief consideration of the basic mechanisms in QP theory. Perhaps contrary to common expectation, the relevant mathematics is simple and mostly based on geometry and linear algebra. We next consider empirical results that appear puzzling from the perspective of CP theory, but can naturally be accommodated within QP models. Finally, we discuss the implications of QP theory for understanding rationality.

\section{Basic assumptions in QP theory and psychological motivation}

\subsection{The outcome space}

CP theory is a set-theoretic way to assign probabilities to the possible outcomes of a question. First, a sample space is defined, in which specific outcomes about a question are subsets of this sample space. Then, a probability measure is postulated, which assigns probabilities to disjoint outcomes in an additive manner (Kolmogorov 1933/ 1950). The formulation is different in QP theory, which is a geometric theory of assigning probabilities to outcomes (Isham 1989). A vector space (called a Hilbert space) is defined, in which possible outcomes are represented as subspaces of this vector space. Note that our use of the terms questions and outcomes are meant to imply the technical QP terms observables and propositions.

A vector space represents all possible outcomes for questions we could ask about a system of interest. For example, consider a hypothetical person and the general question of that person's emotional state. Then, one-dimensional subspaces (called rays) in the vector space would correspond to the most elementary emotions possible. The number of unique elementary emotions and their relation to each other determine the overall dimensionality of the vector space. Also, more general emotions, such as happiness, would be represented by subspaces of higher dimensionality. In Figure 1a, we consider the question of whether a (a)

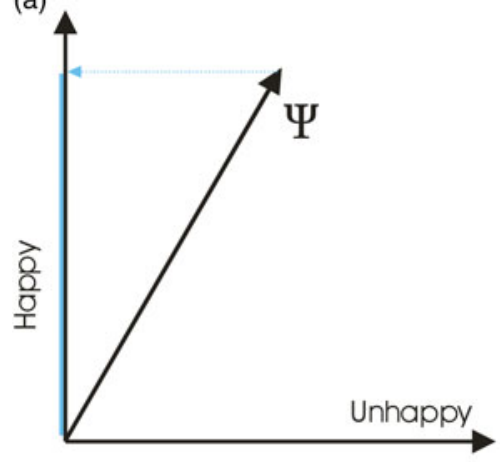

(b)

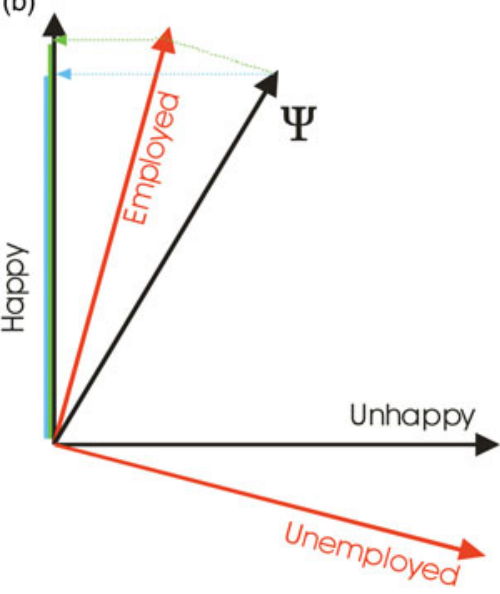

(c)

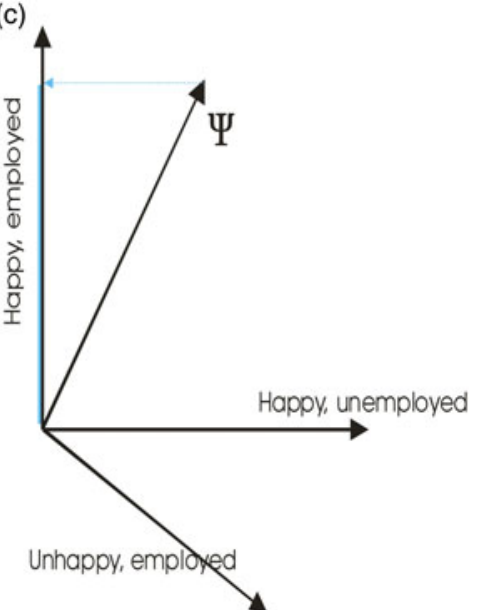

Figure 1. An illustration of basic processes in QP theory. In Figure 1b, all vectors are co-planar, and the figure is a two-dimensional one. In Figure 1c, the three vectors "Happy, employed," "Happy, unemployed," and "Unhappy, employed” are all orthogonal to each other, so that the figure is a three-dimensional one. (The fourth dimension, "unhappy, unemployed" is not shown). 
hypothetical person is happy or not. However, because it is hard to picture high multidimensional subspaces, for practical reasons we assume that the outcomes of the happiness question are one-dimensional subspaces. Therefore, one ray corresponds to the person definitely being happy and another one to that person definitely being unhappy.

Our initial knowledge of the hypothetical person is indicated by the state vector, a unit length vector, denoted as $|\Psi\rangle$ (the bracket notation for a vector is called the Dirac notation). In psychological applications, it often refers to the state of mind, perhaps after reading some instructions for a psychological task. More formally, the state vector embodies all our current knowledge of the cognitive system under consideration. Using the simple vector space in Figure la, we can write $|\Psi\rangle=a \mid$ happy $\rangle+b \mid$ unhappy $\rangle$. Any vector $|\Psi\rangle$ can be expressed as a linear combination of the $\mid$ happy $\rangle$ and |unhappy $\rangle$ vectors, so that these two vectors form a basis for the two-dimensional space we have employed. The $a$ and $b$ constants are called amplitudes and they reflect the components of the state vector along the different basis vectors.

To determine the probability of the answer happy, we need to project the state represented by $|\Psi\rangle$ onto the subspace for "happy" spanned by the vector |happy what is called a projector, which takes the vector $|\Psi\rangle$ and lays it down on the subspace spanned by |happy $\rangle$; this projector can be denoted as $P_{\text {happy }}$. The projection to the |happy subspace is denoted by $P_{\text {happy }}|\Psi\rangle=a \mid$ happy $\rangle$. (Here and elsewhere we will slightly elaborate on some of the basic definitions in the Appendix.) Then, the probability that the person is happy is equal to the squared length of the projection, $\| P_{\text {happy }}|\Psi\rangle \|^{2}$. That is, the probability that the person has a particular property depends upon the projection of $|\Psi\rangle$ onto the subspace corresponding to the property. In our simple example, this probability reduces to $\| P_{\text {happy }}|\Psi\rangle \|^{2}=|a|^{2}$, which is the squared magnitude of the amplitude of the state vector along the |happy $\rangle$ basis vector. The idea that projection can be employed in psychology to model the match between representations has been explored before (Sloman 1993), and the QP cognitive program can be seen as a way to generalize these early ideas. Also, note that a remarkable mathematical result, Gleason's theorem, shows that the QP way for assigning probabilities to subspaces is unique (e.g., Isham 1989, p. 210). It is not possible to devise another scheme for assigning numbers to subspaces that satisfy the basic requirements for an additive probability measure (i.e., that the probabilities assigned to a set of mutually exclusive and exhaustive outcomes are individually between 0 and 1 , and sum to 1 ).

An important feature of QP theory is the distinction between superposition and basis states. In the abovementioned example, after the person has decided that she is happy, then the state vector is $|\Psi\rangle=\mid$ happy $\rangle$; alternatively if she decides that she is unhappy, then $|\Psi\rangle=\mid$ unhappy $\rangle$. These are called basis states, with respect to the question about happiness, because the answer is certain when the state vector $|\Psi\rangle$ exactly coincides with one basis vector. Note that this explains why the subspaces corresponding to mutually exclusive outcomes (such as being happy and being unhappy) are at right angles to each other. If a person is definitely happy, i.e., $|\Psi\rangle=\mid$ happy $\rangle$, then we want a zero probability that the person is unhappy, which means a zero projection to the subspace for unhappy.
This will only be the case if the happy, unhappy subspaces are orthogonal.

Before the decision, the state vector is a superposition of the two possibilities of happiness or unhappiness, so that $|\Psi\rangle=a \mid$ happy $\rangle+b \mid$ unhappy $\rangle$. The concept of superposition differs from the CP concept of a mixed state. According to the latter, the person is either exactly happy or exactly unhappy, but we don't know which, and so we assign some probability to each possibility. However, in QP theory, when a state vector is expressed as $|\Psi\rangle=a$ $\mid$ happy $\rangle+b \mid$ unhappy $\rangle$ the person is neither happy nor unhappy. She is in an indefinite state regarding happiness, simultaneously entertaining both possibilities, but being uncommitted to either. In a superposition state, all we can talk about is the potential or tendency that the person will decide that she is happy or unhappy. Therefore, a decision, which causes a person to resolve the indefinite state regarding a question into a definite (basis) state, is not a simple read-out from a pre-existing definite state; instead, it is constructed from the current context and question (Aerts \& Aerts 1995). Note that other researchers have suggested that the way of exploring the available premises can affect the eventual judgment, as much as the premises themselves, so that judgment is a constructive process (e.g., Johnson et al. 2007; Shafer \& Tversky 1985). The interesting aspect of QP theory is that it fundamentally requires a constructive role for the process of disambiguating a superposition state (this relates to the Kochen-Specker theorem).

\subsection{Compatibility}

Suppose that we are interested in two questions, whether the person is happy or not, and also whether the person is employed or not. In this example, there are two outcomes with respect to the question about happiness, and two outcomes regarding employment. In CP theory, it is always possible to specify a single joint probability distribution over all four possible conjunctions of outcomes for happiness and employment, in a particular situation. (Griffiths [2003] calls this the unicity principle, and it is fundamental in CP theory). By contrast, in QP theory, there is a key distinction between compatible and incompatible questions. For compatible questions, one can specify a joint probability function for all outcome combinations and in such cases the predictions of CP and QP theories converge (ignoring dynamics). For incompatible questions, it is impossible to determine the outcomes of all questions concurrently. Being certain about the outcome of one question induces an indefinite state regarding the outcomes of other, incompatible questions.

This absolutely crucial property of incompatibility is one of the characteristics of QP theory that differentiates it from CP theory. Psychologically, incompatibility between questions means that a cognitive agent cannot formulate a single thought for combinations of the corresponding outcomes. This is perhaps because that agent is not used to thinking about these outcomes together, for example, as in the case of asking whether Linda (Tversky \& Kahneman 1983) can be both a bank teller and a feminist. Incompatible questions need to be assessed one after the other. A heuristic guide of whether some questions should be considered compatible is whether clarifying one is expected to interfere with the evaluation of the other. Psychologically, the 
Pothos \& Busemeyer: Can quantum probability provide a new direction for cognitive modeling?

intuition is that considering one question alters our state of mind (the context), which in turn affects consideration of the second question. Therefore, probability assessment in QP theory can be (when we have incompatible questions) order and context dependent, which contrasts sharply with CP theory.

Whether some questions are considered compatible or incompatible is part of the analysis that specifies the corresponding cognitive model. Regarding the questions for happiness and employment for the hypothetical person, the modeler would need to commit a priori as to whether these are compatible or incompatible. We consider in turn the implications of each approach.

2.2.1. Incompatible questions. For outcomes corresponding to one-dimensional subspaces, incompatibility means that subspaces exist at nonorthogonal angles to each other, as in, for example, for the happy and employed subspaces in Figure 1b. Because of the simple relation we assume to exist between happiness and employment, all subspaces can be coplanar, so that the overall vector space is only two dimensional. Also, recall that certainty about a possible outcome in QP theory means that the state vector is contained within the subspace for the outcome. For example, if we are certain that the person is happy, then the state vector is aligned with the happy subspace. However, if this is the case, we can immediately see that we have to be somewhat uncertain about the person's employment (perhaps thinking about being happy makes the person a bit anxious about her job). Conversely, certainty about employment aligns the state vector with the subspace for employed, which makes the person somewhat uncertain about her happiness (perhaps her job is sometimes stressful). This is a manifestation of the famous Heisenberg uncertainty principle: Being clear on one question forces one to be unclear on another incompatible question.

Because it is impossible to evaluate incompatible questions concurrently, quantum conjunction has to be defined in a sequential way, and so order effects may arise in the overall judgment. For example, suppose that the person is asked first whether she is employed, and then whether she is happy, that is, we have

$$
\begin{aligned}
\operatorname{Prob}(\text { employed } \wedge \text { then happy })= & \operatorname{Prob}(\text { employed }) \\
& \cdot \operatorname{Prob}(\text { happy } \mid \text { employed })
\end{aligned}
$$

whereby the first term is

$$
\operatorname{Prob}(\text { employed })=\| P_{\text {employed }}|\psi\rangle \|^{2}
$$

The second term is the probability that the person is happy, given that the person is employed. Certainty that the person is employed means that the state vector is

$$
\left.\| \psi_{\text {employed }}\right\rangle=\frac{p_{\text {employed }}|\psi\rangle}{\| p_{\text {employed }}|\psi\rangle \|}
$$

Therefore

$$
\operatorname{Prob}(\text { happy|employed })=\| P_{\text {happy }}\left|\psi_{\text {employed }}\right\rangle \|^{2}
$$

which leads to

$$
\operatorname{Prob}(\text { employed } \wedge \text { then happy })=\| P_{\text {happy }} P_{\text {employed }}|\psi\rangle \|^{2}
$$

Therefore, in QP theory, a conjunction of incompatible questions involves projecting first to a subspace corresponding to an outcome for the first question and, second, to a subspace for the second question (Busemeyer et al. 2011). This discussion also illustrates the QP definition for conditional probability, which is in general

$$
\operatorname{Prob}(A \mid B)=\frac{\| P_{A} P_{B}|\psi\rangle \|^{2}}{\| P_{B}|\psi\rangle \|^{2}}=\frac{\operatorname{Prob}(B \wedge \text { then } A)}{\operatorname{Prob}(B)}
$$

(this is called Lüder's law).

It is clear that the definition of conditional probability in QP theory is analogous to that in CP theory, but for potential order effects in the sequential projection $P_{A} P_{B}$, when $A$ and $B$ are incompatible.

The magnitude of a projection depends upon the angle between the corresponding subspaces. For example, when the angle is large, a lot of amplitude is lost between successive projections. As can be seen in Figure 1b,

$$
\| P_{\text {happy }}|\psi\rangle\left\|^{2}<\right\| P_{\text {happy }} P_{\text {employed }}|\psi\rangle \|^{2}
$$

that is, the direct projection to the happy subspace (green line) is less than the projection to the happy subspace via the employed one (light blue line). (Color versions of the figures in this article are available at http://dx.doi.org/10. 1017/S0140525X12001525].) The psychological intuition would be that if the person is asked whether she is employed or not, and concludes that she is, perhaps this makes her feel particularly good about herself, which makes it more likely that she will say she is happy. In classical terms, here we have a situation whereby

$$
\operatorname{Prob}(\text { happy })<\operatorname{Prob}(\text { happy } \wedge \text { employed })
$$

which is impossible in CP theory. Moreover, consider the comparison between first asking "are you employed" and then "are you happy" versus first asking "are you happy" and then "are you employed." In CP theory, this corresponds to

$$
\operatorname{Prob}(\text { employed } \wedge \text { happy })=\operatorname{Prob}(\text { happy } \wedge \text { employed }) .
$$

However, in QP theory conjunction of incompatible questions fails commutativity. We have seen that

$$
\operatorname{Prob}(\text { employed } \wedge \text { then happy })=\| P_{\text {happy }} P_{\text {employed }}|\psi\rangle \|^{2}
$$

is large. By contrast, 
Pothos \& Busemeyer: Can quantum probability provide a new direction for cognitive modeling?

$\operatorname{Prob}($ happy $\wedge$ then employed $)=\| P_{\text {employed }} P_{\text {happy }}|\psi\rangle \|^{2}$

is less large, because in this case we project from $|\Psi\rangle$ to $|h a p p y\rangle$, whereby we lose quite a bit of amplitude (their relative angle is large) and then from $\mid$ happy $\rangle$ to |employed $\rangle$ (we lose more amplitude).

In general, the smaller the angle between the subspaces for two incompatible outcomes, the greater the relation between the outcomes. A small angle is analogous to a high correlation in a classical framework. When there is a small angle, a sequential projection of the state vector from one subspace to the other loses little amplitude. Accordingly, accepting one outcome makes the other outcome very likely as well. The size of such angles and the relative dimensionality of the subspaces are the cornerstones of QP cognitive models and are determined by the known psychology of the problem. These angles (and the initial state vector) have a role in QP theory analogous to that of prior and conditional distributions in Bayesian modeling. In the toy illustration of Figure 1b, the only guidance in placing the subspaces is that the employed and happy subspaces should be near each other, to reflect the expectation that employment tends to relate to happiness. The state vector was placed near the employed subspace, assuming the person is confident in her employment.

Note that the above discussion does not concern probabilistic assessments indexed by time. That is, we are not comparing

Prob(employed on Monday ^ happy on Tuesday)

versus

Prob(happy on Monday ^ employed on Tuesday).

Both CP and QP theories predict these to be different, because the events are distinguished by time, so we no longer compare the same events ("employed on Monday" is not the same event as "employed on Tuesday"). Rather, here we are concerned with the order of assessing a combination of two events, when the two events are defined in exactly the same way. But could order dependence in quantum theory arise as probability dependence in classical theory? The answer is no because

$$
\begin{aligned}
\operatorname{Prob}(A \wedge B) & =\operatorname{Prob}(A) \operatorname{Prob}(B \mid A)=\operatorname{Prob}(B) \operatorname{Prob}(A \mid B) \\
& =\operatorname{Prob}(B \wedge A)
\end{aligned}
$$

In quantum theory, the intermediate step is not possible whenever $P_{A} P_{B} \neq P_{B} P_{A}$. Note that in an expressions such as

$$
\operatorname{Prob}(\text { employed } \wedge \text { then happy })=\| P_{\text {happy }} P_{\text {employed }}|\psi\rangle \|^{2}
$$

there are two sources of uncertainty. There is the classical uncertainty about the various outcomes. There is a further uncertainty as to how the state will collapse after the first question (if the two questions are incompatible). This second source of uncertainty does not exist in a classical framework, as classically it is assumed that a measurement (or evaluation) simply reads off existing values. By contrast, in quantum theory a measurement can create a definite value for a system, which did not previously exist (if the state of the system was a superposition one).

We have seen how it is possible in QP theory to have definite knowledge of one outcome affect the likelihood of an alternative, incompatible outcome. Order and context dependence of probability assessments (and, relatedly, the failure of commutativity in conjunction) are some of the most distinctive and powerful features of QP theory. Moreover, the definitions for conjunction and conditional probability in QP theory are entirely analogous to those in CP theory, except for the potential of order effects for incompatible questions.

2.2.2. Compatible questions. Now assume that the happiness and employment questions are compatible, which means that considering one does not influence consideration of the other, and all four possible conjunctions of outcomes are defined. To accommodate these outcome combinations, we need a four-dimensional space, in which each basis vector corresponds to a particular combination of happiness and employment outcomes (Figure 1c is a three-dimensional simplification of this space, leaving out the fourth dimension). Then, the probability that the person is happy and employed is given by projecting the state vector onto the corresponding basis vector. Clearly,

$$
\begin{aligned}
\operatorname{Prob}(\text { happy } \wedge \text { employed }) & =\| P_{\text {happy } \wedge \text { employed }}|\psi\rangle \|^{2} \\
& =\operatorname{Prob}(\text { employed } \wedge \text { happy }) .
\end{aligned}
$$

Thus, for compatible questions, conjunction is commutative, as in CP theory.

The vector space for compatible outcomes is formed by an operation called a tensor product, which provides a way to construct a composite space out of simpler spaces. For example, regarding happiness we can write

$$
|H\rangle=h \cdot|h a p p y\rangle+h^{\prime} \cdot|\sim h a p p y\rangle
$$

and this state vector allows us to compute the probability that the person is happy or not. Likewise, regarding employment, we can write

$$
\left.|E\rangle=e \cdot \mid \text { employed }\rangle+e^{\prime} \cdot \mid \sim \text { employed }\right\rangle .
$$

As long as happiness and employment are compatible, the tensor product between $|H\rangle$ and $|E\rangle$ is given by

$$
\begin{aligned}
& \mid \text { product state }\rangle=|H\rangle \otimes|E\rangle \\
& \left.=h \cdot e \cdot \mid \text { happy }\rangle \otimes \mid \text { employed }\rangle+h \cdot e^{\prime} \cdot \mid \text { happy }\right\rangle \\
& \left.\left.\quad \otimes \mid \sim \text { employed }\rangle+h^{\prime} \cdot e \cdot \mid \sim \text { happy }\right\rangle \otimes \mid \text { employed }\right\rangle \\
& \left.\left.\quad+h^{\prime} \cdot e^{\prime} \cdot \mid \sim \text { happy }\right\rangle \otimes \mid \sim \text { employed }\right\rangle .
\end{aligned}
$$

This four-dimensional product state is formed from the basis vectors representing all possible combinations of whether the person is employed or not and is happy or not. For example, $\mid$ happy $\rangle \otimes|e m p l o y e d\rangle \mid$ or for brevity 
Pothos \& Busemeyer: Can quantum probability provide a new direction for cognitive modeling?

$|h a p p y\rangle|e m p l o y e d\rangle$, denotes a single basis vector that represents the occurrence of the conjunction "happy and employed" (Figure 1c). The joint probability that the person is employed and happy simply equals $|h \cdot e|^{2}$. This probability agrees with the classical result for Prob (employed $\wedge$ happy), in the sense that the QP conjunction is interpreted (and has the same properties) as conjunction in CP theory.

What are the implications for psychological modeling? Tensor product representations provide a concrete and rigorous way of creating structured spatial representations in QP theory. Several researchers have pointed out that representations for even the most basic concepts must be structured, as information about the different elements of a concept are compared to like (alignable) elements in an alternative concept (Goldstone 1994; Hahn et al. 2003; Markman \& Gentner 1993). Such intuitions can be readily realized in a QP framework through tensor product representations. Note that this idea is not new: others have sought to develop structured representations via tensor products (Smolensky 1990). The advantage of QP theory is that a tensor product representation is supported by a framework for assessing probabilities.

CP theory is also consistent with structured representations. However, in QP theory, because of the property of superposition, creating structured representations sometimes leads to a situation of entanglement. Entanglement relates to some of the most puzzling properties of QP theory. To explain it, we start from a state that is not entangled, the |product state $\rangle$ described earlier, and assume that the person is definitely employed $(e=1)$, so that the state reduces to

$$
\begin{aligned}
\mid \text { reduced state }\rangle= & h \cdot \mid \text { happy }\rangle \mid \text { employed }\rangle \\
& \left.\left.+h^{\prime} \cdot \mid \sim \text { happy }\right\rangle \mid \text { employed }\right\rangle .
\end{aligned}
$$

So far, we can see how the part for being happy is completely separate from the part for being employed. It should be clear that in such a simple case, the probability of being happy is independent (can be decomposed from) the probability of being employed. As long as the state vector has a product form (e.g., as mentioned), the components for each subsystem can be separated out. This situation is entirely analogous to that in CP theory for independent events, whereby a composite system can always be decomposed into the product of its separate subsystems.

An entangled state is one for which it is not possible to write the state vector as a tensor product between two vectors. Suppose we have

$$
\begin{aligned}
\mid \text { entangled state }\rangle= & x \cdot \mid \text { happy }\rangle \mid \text { employed }\rangle \\
& +w \cdot \mid \sim \text { happy }\rangle \mid \sim \text { employed }\rangle .
\end{aligned}
$$

This |entangled state $\rangle$ does not correspond to either a decision being made regarding being happy or a clarification regarding employment. Such states are called entangled states, because an operation that influences one part of the system (e.g., being happy), inexorably affects the other (clarifying employment). In other words, in such an entangled state, the possibilities of being happy and employed are strongly dependent upon each other. The significance of entanglement is that it can lead to an extreme form of dependency between the outcomes for a pair of questions, which goes beyond what is possible in CP theory. In classical theory, one can always construct a joint probability $\operatorname{Prob}(A, B, C)$ out of pairwise ones, and $\operatorname{Prob}(A, B), \operatorname{Prob}(A, C)$, and $\operatorname{Prob}(B, C)$ are all constrained by this joint. However, in QP theory, for entangled systems, it is not possible to construct a complete joint, because the pairwise probabilities can be stronger than what is allowed classically (Fine 1982).

\subsection{Time evolution}

So far, we have seen static QP models, whereby we assess the probability for various outcomes for a state at a single point in time. We next examine how the state can change in time. Time evolution in QP theory involves a rotation (technically, a unitary) operator (the solution to Schrödinger's equation). This dynamic operator evolves the initial state vector, without changing its magnitude. It is important to recall that the state vector is a superposition of components along different basis vectors. Therefore, what evolves are the amplitudes along the different basis vectors. For example, a rotation operator might move the state $|\Psi\rangle$ away from the | happy $\rangle$ basis vector toward the |unhappy $\rangle$ one, if the modeled psychological process causes unhappiness with time. Analogously, time evolution in CP theory involves a transition matrix (the solution to Kolmogorov's forward equation). The classical initial state corresponds to a joint probability distribution over all combinations of outcomes. Time evolution involves a transformation of these probabilities, without violating the law of total probability.

In both CP and QP theories, time evolution corresponds to a linear transformation of the initial state. In CP theory, the time-evolved state directly gives the probabilities for the possible outcomes. Time evolution is a linear transformation that preserves the law of total probability. By contrast, in QP theory, whereas the state vector amplitudes are linearly transformed, probabilities are obtained by squaring the length of the state vector. This nonlinearity means that the probabilities obtained from the initial state vector may obey the law of total probability, but this does not have to be the case for the time-evolved ones. Therefore, in QP theory, time evolution can produce probabilities that violate the law of total probability. This is a critical difference between CP and QP theory and argues in favor of the latter, to the extent that there are cognitive violations of the law of total probability.

As an example, suppose the hypothetical person is due a major professional review and she is a bit anxious about continued employment (so that she is unsure about whether she is employed or not). Prior to the review, she contemplates whether she is happy to be employed or not. In this example, we assume that the employment and happiness questions are compatible (Figure 1c). In $\mathrm{CP}$ theory, the initial probabilities satisfy

$$
\begin{aligned}
\operatorname{Prob}(\text { happy, unknown empl. })= & \operatorname{Prob}(\text { happy } \wedge \text { employed }) \\
& +\operatorname{Prob}(\text { happy } \wedge \text { not employed }) .
\end{aligned}
$$

Next, assume that the state vector evolves for time $t$. This process of evolution could correspond, for example, to the 
Pothos \& Busemeyer: Can quantum probability provide a new direction for cognitive modeling?

thought process of considering happiness, depending upon employment assumptions. It would lead to a final set of probabilities that satisfy

$$
\begin{aligned}
& \text { Prob(happy, unknown empl., at } t) \\
& =\text { Prob(happy at } t \wedge \text { employed }) \\
& \quad+\text { Prob(happy at } t \wedge \text { notemployed })
\end{aligned}
$$

Although the final distribution differs from the initial distribution, they both obey the law of total probability. In QP theory, we can write the initial state vector as

$$
\begin{aligned}
\text { State }(\text { happy, unknown empl. })= & \text { State }(\text { happy } \wedge \text { employed }) \\
& +(\text { happy } \wedge \text { not employed }) .
\end{aligned}
$$

After time evolution, we have

$$
\begin{aligned}
& \text { State }(\text { happy, unknownempl., at } t) \\
& =\text { State }(\text { happy at } t \wedge \text { employed }) \\
& \quad+\text { State }(\text { happy at } t \wedge \text { not employed })
\end{aligned}
$$

but

$$
\begin{aligned}
& \text { Prob(happy, unknown empl., at } t) \\
& =\text { Prob(happy at } t \wedge \text { employed) } \\
& \quad+\text { Prob(happy at } t \wedge \text { not employed) } \\
& \quad+\text { Interference (crossproduct) terms }
\end{aligned}
$$

(see Appendix). One way in which interference effects can arise in QP theory is by starting with a state vector that is a superposition of orthogonal states. Then, time evolution can result in the state vector being a superposition of states, which are no longer orthogonal. As quantum probabilities are determined from the state vector by squaring its length, we have a situation analogous to $|a+b|^{2}=a^{2}+$ $b^{2}+a^{*} b+b^{*} a$. When the states corresponding to $a, b$ are orthogonal, the interference terms $a^{*} b+b^{*} a$ disappear and QP theory reduces to CP theory. Otherwise, QP theory can produce violations of the law of total probability.

Interference terms can be positive or negative and their particular form will depend upon the specifics of the corresponding model. In the previous example, negative interference terms could mean that the person may think she would be happy if it turns out she is employed (perhaps because of the extra money) or that she would be happy if she loses her job (perhaps she doesn't like the work). However, when she is unsure about her employment, she becomes unhappy. It is as if these two individually good reasons for being happy cancel each other out (Busemeyer \& Bruza 2012, Ch. 9). That a preference that is dominant under any single definite condition can be reversed in an unknown condition is a remarkable feature of QP theory and one that (as will be discussed) corresponds well to intuition about psychological process (Tversky \& Shafir 1992).

Suppose that the hypothetical person knows she will find out whether she will be employed or not, before having the inner reflection about happiness (perhaps she plans to think about her happiness after a professional review). The resolution regarding employment eliminates any possible interference effects from her judgment, and the quantum prediction converges to the classical one (Appendix). Therefore, in QP theory, there is a crucial difference between (just) uncertainty and superposition and it is only the latter that can lead to violations of the law of total probability. In quantum theory, just the knowledge that an uncertain situation has been resolved (without necessarily knowing the outcome of the resolution) can have a profound influence on predictions.

\section{The empirical case for QP theory in psychology}

In this section, we explore whether the main characteristics of QP theory (order/context effects, interference, superposition, entanglement) provide us with any advantage in understanding psychological processes. Many of these situations concern Kahneman and Tversky's hugely influential research program on heuristics and biases (Kahneman et al. 1982; Tversky \& Kahneman 1973; 1974; 1983), one of the few psychology research programs to have been associated with a Nobel prize (in economics, for Kahneman in 2002). This research program was built around compelling demonstrations that key aspects of CP theory are often violated in decision making and judgment. Therefore, this is a natural place to start looking for whether QP theory may have an advantage over CP theory.

Our strategy is to first discuss how the empirical finding in question is inconsistent with CP theory axioms. This is not to say that some model broadly based on classical principles cannot be formulated. Rather, that the basic empirical finding is clearly inconsistent with classical principles and that a classical formalism, when it exists, may be contrived. We then present an illustration for how a QP approach can offer the required empirical coverage. Such illustrations will be simplifications of the corresponding quantum models.

\subsection{Conjunction fallacy}

In a famous demonstration, Tversky and Kahneman (1983) presented participants with a story about a hypothetical person, Linda, who sounded very much like a feminist. Participants were then asked to evaluate the probability of statements about Linda. The important comparison concerned the statements "Linda is a bank teller" (extremely unlikely given Linda's description) and "Linda is a bank teller and a feminist." Most participants chose the second statement as more likely than the first, thus effectively judging that

$$
\operatorname{Prob}(\text { bank teller })<\operatorname{Prob}(\text { bank teller } \wedge \text { feminist }) \text {. }
$$

This critical empirical finding is obtained with different kinds of stories or dependent measures (including betting procedures that do not rely on the concept of probability; Gavanski \& Roskos-Ewoldsen 1991; Sides et al. 2002; Stolarz-Fantino et al. 2003; Tentori \& Crupi 2012; Wedell \& Moro 2008). However, according to CP theory this is impossible, because the conjunction of two statements can never be more probable than either statement individually (this finding is referred to as the conjunction fallacy). The $\mathrm{CP}$ intuition can be readily appreciated in frequentist terms: in a sample space of all possible Lindas, of the 
Pothos \& Busemeyer: Can quantum probability provide a new direction for cognitive modeling?

ones who are bank tellers, only a subset will be both bank tellers and feminists. Tversky and Kahneman's explanation was that (classical) probability theory is not appropriate for understanding such judgments. Rather, such processes are driven by a similarity mechanism, specifically a representativeness heuristic, according to which participants prefer the statement "Linda is a bank teller and a feminist" because Linda is more representative of a stereotypical feminist. A related explanation, based on the availability heuristic, is that the conjunctive statement activates memory instances similar to Linda (Tversky \& Koehler 1994).

QP theory provides an alternative way to understand the conjunction fallacy. In Figure 2, we specify $|\Psi\rangle$, the initial state vector, to be very near the basis vector for $\mid$ feminist $\rangle$ and nearly orthogonal to the basis vector for |bank teller $\rangle$. Also, the $\mid$ feminist $\rangle$ basis vector is neither particularly close nor particularly far away from the |bank teller $\rangle$ one, because to be a bank teller is not perhaps the most likely profession for feminists, but it is not entirely unlikely either. These are our priors for the problem, that is, that the description of Linda makes it very likely that she is a feminist and very unlikely that she is a bank teller. Note the limited flexibility in the specification of these subspaces and the state vector. For example, the state vector could not be placed in between the bank teller and feminist subspaces, as this would mean that it is has a high projection to both the bank teller and the feminist outcomes (only the latter is true). Likewise, it would make no sense to place the feminist subspace near the bank teller one, or to the not bank teller one, as feminism is a property that is largely uninformative as to whether a person is a bank teller or not.

Consider the conjunctive statement "Linda is a bank teller and a feminist." As we have seen, in QP theory,

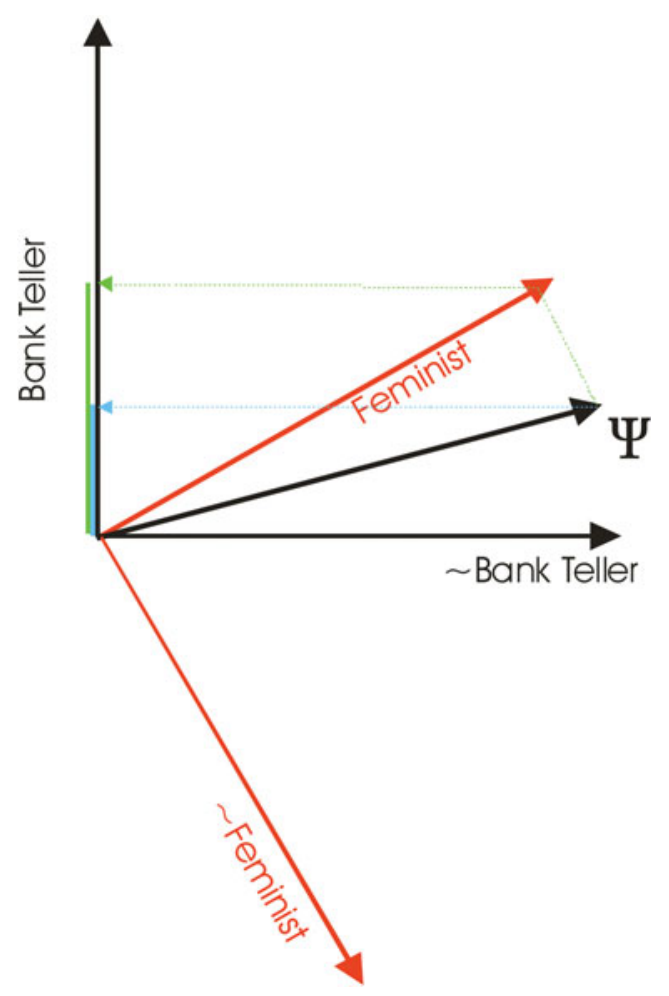

Figure 2. An illustration of the QP explanation for the conjunction fallacy. conjunctions are evaluated as sequences of projections. An additional assumption is made that in situations such as this, the more probable possible outcome is evaluated first (this is a reasonable assumption, as it implies that more probable outcomes are prioritized in the decision making process; cf. Gigerenzer \& Todd 1999). Therefore, the conjunctive statement involves first projecting onto the feminist basis vector, and subsequently projecting on the bank teller one. It is immediately clear that this sequence of projections leads to a larger overall amplitude (green line), compared to the direct projection from $|\Psi\rangle$ onto the bank teller vector.

Psychologically, the QP model explains the conjunction fallacy in terms of the context dependence of probability assessment. Given the information participants receive about Linda, it is extremely unlikely that she is a bank teller. However, once participants think of Linda in more general terms as a feminist, they are more able to appreciate that feminists can have all sorts of professions, including being bank tellers. The projection acts as a kind of abstraction process, so that the projection onto the feminist subspace loses some of the details about Linda, which previously made it impossible to think of her as a bank teller. From the more abstract feminist point of view, it becomes a bit more likely that Linda could be a bank teller, so that whereas the probability of the conjunction remains low, it is still more likely than the probability for just the bank teller property. Of course, from a QP theory perspective, the conjunctive fallacy is no longer a fallacy, it arises naturally from basic QP axioms.

Busemeyer et al. (2011) presented a quantum model based on this idea and examined in detail the requirements for the model to predict an overestimation of conjunction. In general, QP theory does not always predict an overestimation of conjunction. However, given the details of the Linda problem, an overestimation of conjunction necessarily follows. Moreover, the same model was able to account for several related empirical findings, such as the disjunction fallacy, event dependencies, order effects, and unpacking effects (e.g., Bar-Hillel \& Neter 1993; Carlson \& Yates 1989; Gavanski \& Roskos-Ewoldsen 1991; Stolarz-Fantino, et al. 2003). Also, the QP model is compatible with the representativeness and availability heuristics. The projection operations used to compute probabilities measure the degree of overlap between two vectors (or subspaces), and overlap is a measure of similarity (Sloman 1993). Thus, perceiving Linda as a feminist allows the cognitive system to establish similarities between the initial representation (the initial information about Linda) and the representation for bank tellers. If we consider representativeness to be a similarity process, as we can do with the QP model, it is not surprising that it is subject to chaining and context effects. Moreover, regarding the availability heuristic (Tversky \& Koehler 1994), the perspective from the QP model is that considering Linda to be a feminist increases availability for other related information about feminism, such as possible professions.

\subsection{Failures of commutativity in decision making}

We next consider failures of commutativity in decision making, whereby asking the same two questions in different orders can lead to changes in response (Feldman \& Lynch 1988; Schuman \& Presser 1981; Tourangeau et al. 
Pothos \& Busemeyer: Can quantum probability provide a new direction for cognitive modeling?

2000). Consider the questions "Is Clinton honest?" and "Is Gore honest?" and the same questions in a reverse order. When the first two questions were asked in a Gallup poll, the probabilities of answering yes for Clinton and Gore were $50 \%$ and $68 \%$, respectively. The corresponding probabilities for asking the questions in the reverse order were, by contrast, $57 \%$ and $60 \%$ (Moore 2002). Such order effects are puzzling according to CP theory, because, as noted, the probability of saying yes to question $A$ and then yes to question $B$ equals

$$
\begin{aligned}
\operatorname{Prob}(A) \cdot \operatorname{Prob}(B \mid A) & =\operatorname{Prob}(A \wedge B)=\operatorname{Prob}(B \wedge A) \\
& =\operatorname{Prob}(B) \cdot \operatorname{Prob}(A \mid B) .
\end{aligned}
$$

Therefore, CP theory predicts that the order of asking two questions does not matter. By contrast, the explanation for order effects in social psychology is that the first question activates thoughts, which subsequently affect consideration of the second question (Schwarz 2007).

QP theory can accommodate order effects in Gallup polls, in a way analogous to how the conjunction fallacy is explained. In both cases, the idea is that the context for assessing the first question influences the assessment of any subsequent questions. Figure 3 is analogous to Figure 2. In Figure 3, there are two sets of basis vectors, one for evaluating whether Clinton is honest or not and another for evaluating whether Gore is honest or not. The two sets of basis vectors are not entirely orthogonal; we assume that if a person considers Clinton honest, then that person is a little more likely to consider Gore to be honest as well, and vice versa (as they ran for office together). The initial state vector is fairly close to the |Gore yes $\rangle$ vector, but less close to the $\mid$ Clinton yes $\rangle$ basis vector, to reflect the information that Gore would be considered more honest than Clinton. The length of the projection onto the |Clinton yes $\rangle$ basis vector reflects the probability that Clinton is honest. It can be seen that the direct projection is less, compared to the projection via the $\mid$ Gore yes $\rangle$ vector. In other words, deciding that Gore is honest increases the probability that Clinton is judged to be honest as well (and, conversely,

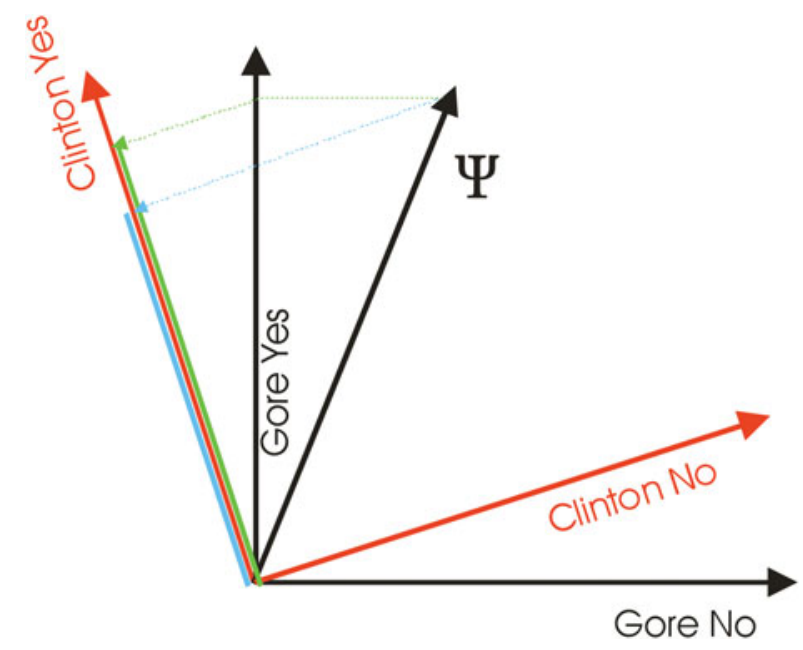

Figure 3. An illustration of order effects in Gallup polls. deciding that Clinton is honest first, reduces the probability that Gore is judged as honest).

The actual QP theory model developed for such failures in commutativity was based on the abovementioned idea, but was more general, so as to provide a parameter free test of the relevant empirical data (e.g., there are various specific types of order effects; Wang \& Busemeyer, in press).

A related failure of commutativity concerns the order of assessing different pieces of evidence for a particular hypothesis. According to CP theory, the order in which evidence $A$ and $B$ is considered, in relation to a hypothesis $H$, is irrelevant, as

$$
\operatorname{Prob}(H \mid A \wedge B)=\operatorname{Prob}(H \mid B \wedge A) .
$$

However, there have been demonstrations that, in fact,

$$
\operatorname{Prob}(H \mid A \wedge B) \neq \operatorname{Prob}(H \mid B \wedge A)
$$

(Hogarth \& Einhorn 1992; Shanteau 1970; Walker et al. 1972). Trueblood and Busemeyer (2011) proposed a QP model for two such situations, a jury decision-making task (McKenzie et al. 2002) and a medical inference one (Bergus et al. 1998). For example, in the medical task participants (all medical practitioners) had to make a decision about a disease based on two types of clinical information. The order of presenting this information influenced the decision, with results suggesting that the information presented last was weighted more heavily (a recency effect). Trueblood and Busemeyer's (2011) model involved considering a tensor product space for the state vector, with one space corresponding to the presence or absence of the disease (this is the event we are ultimately interested in) and the other space to positive or negative evidence, evaluated with respect to the two different sources of information (one source of information implies positive evidence for the disease and the other negative evidence). Considering each source of clinical information involved a rotation of the state vector, in a way reflecting the impact of the information on the disease hypothesis. The exact degree of rotation was determined by free parameters. Using the same number of parameters, the QP theory model produced better fits to empirical results than the anchoring and adjustment model of Hogarth and Einhorn (1992) for the medical diagnosis problem and for the related jury decision one.

\subsection{Violations of the sure thing principle}

The model Trueblood and Busemeyer (2011) developed is an example of a dynamic QP model, whereby the inference process requires evolution of the state vector. This same kind of model has been employed by Pothos and Busemeyer (2009) and Busemeyer et al. (2009) to account for violations of the sure thing principle. The sure thing principle is the expectation that human behavior ought to conform to the law of total probability. For example, in a famous demonstration, Shafir and Tversky (1992) reported that participants violated the sure thing principle in a oneshot prisoner's dilemma task. This is a task whereby participants receive different payoffs depending upon whether they decide to cooperate or defect, relative to another 
Pothos \& Busemeyer: Can quantum probability provide a new direction for cognitive modeling?

(often hypothetical) opponent. Usually the player does not know the opponents' move, but in some conditions Shafir and Tversky told participants what the opponent had decided to do. When participants were told that the opponent was going to cooperate, they decided to defect; and when they were told that the opponent was defecting, they decided to defect as well. The payoffs were specified in such a way so that defection was the optimal strategy. The expectation from the sure thing principle is that, when no information was provided about the action of the opponent, participants should also decide to defect (it is a "sure thing" that defection is the best strategy, because it is the best strategy in all particular cases of opponent's actions). However, surprisingly, in the "no knowledge" case, many participants reversed their judgment and decided to cooperate (Busemeyer et al. 2006a; Croson 1999; Li \& Taplin 2002). Similar results have been reported for the two-stage gambling task (Tversky \& Shafir 1992) and a novel categorization-decisionmaking paradigm (Busemeyer et al. 2009; Townsend et al. 2000). Therefore, violations of the sure thing principle in decision making, although relatively infrequent, are not exactly rare either. Note that this research has established violations of the sure thing principle using within-participants designs.

Shafir and Tversky (1992) suggested that participants perhaps adjust their beliefs for the other player's action, depending upon what they are intending to do (this principle was called wishful thinking and follows from cognitive dissonance theory and related hypotheses, e.g., Festinger 1957; Krueger et al. 2012). Therefore, if there is a slight bias for cooperative behavior, in the unknown condition participants might be deciding to cooperate because they imagine that the opponent would cooperate as well. Tversky and Shafir (1992) described such violations of the sure thing principle as failures of consequential reasoning. When participants are told that the opponent is going to defect, they have a good reason to defect as well, and, likewise, when they are told that the opponent is going to cooperate. However, in the unknown condition, it is as if these (separate) good reasons for defecting under each known condition cancel each other out (Busemeyer \& Bruza 2011, Ch. 9).

This situation is similar to the generic example for violations of the law of total probability that we considered in Section 2. Pothos and Busemeyer (2009) developed a quantum model for the two-stage gambling task and prisoner's dilemma embodying these simple ideas. A state vector was defined in a tensor product space of two spaces, one corresponding to the participant's intention to cooperate or defect and one for the belief of whether the opponent is cooperating or defecting. A unitary operator was then specified to rotate the state vector depending on the payoffs, increasing the amplitudes for those combinations of action and belief maximizing payoff. The same unitary operator also embodied the idea of wishful thinking, rotating the state vector so that the amplitudes for the "cooperate-cooperate" and "defect-defect" combinations for participant and opponent actions increased. Thus, the state vector developed as a result of two influences. The final probabilities for whether the participant is expected to cooperate or defect were computed from the evolved state vector, by squaring the magnitudes of the relevant amplitudes.
Specifically, the probability of defecting when the opponent is known to defect is based on the projection $P_{\text {participant to } D}\left|\Psi_{\text {opponent known } D}\right\rangle$, where $P_{\text {participant to } D}$ is a projection operator corresponding to the participant choosing to defect. Similarly, the probability of defecting when the opponent is known to cooperate is based on the projection $P_{\text {participant to } D}\left|\Psi_{\text {opponent known } C}\right\rangle$. But, in the unknown case, the relevant state vector is the superposition $\frac{1}{\sqrt{2}}\left|\psi_{\text {opponent known } D}\right\rangle+\frac{1}{\sqrt{2}}\left|\psi_{\text {opponent known } C}\right\rangle$. The probability for the participant to defect is computed by first using the operator $P_{\text {participant to } D}$ on this superposition, which gives us $P_{\text {participant to } D}\left(\left|\Psi_{\text {opponent known } D}\right\rangle+\left|\Psi_{\text {opponent known } C}\right\rangle\right)$, and subsequently squaring the length of the resulting projection. Therefore, we have another case of $|a+b|^{2}=a^{2}+$ $b^{2}+a^{*} b+b^{*} a$, with non-zero interference terms. Thus, a high probability to defect in the two known conditions (high $a^{2}$ and high $b^{2}$ ) can be offset by negative interference terms, which means a lower probability to defect in the unknown condition. We can interpret these computations in terms of Tversky and Shafir's (1992) description of the result as a failure of consequential reasoning. Moreover, the QP model provides a formalization of the wishful thinking hypothesis, with the specification of a corresponding unitary operator matrix. However, note that this quantum model is more complex than the ones considered previously. It requires more detail to see how interference arises, in a way that leads to the required result, and the model involves two parameters (model predictions are robust across a wide range of parameter space).

\subsection{Asymmetry in similarity}

We have considered how the QP explanation for the conjunction fallacy can be seen as a formalization of the representativeness heuristic (Tversky \& Kahneman 1983). This raises the possibility that the QP machinery could be employed for modeling similarity judgments. In one of the most influential demonstrations in the similarity literature, Tversky (1977) showed that similarity judgments violate all metric axioms. For example, in some cases, the similarity of $A$ to $B$ would not be the same as the similarity of $B$ to A. Tversky's (1977) findings profoundly challenged the predominant approach to similarity, whereby objects are represented as points in a multidimensional space, and similarity is modeled as a function of distance. Since then, novel proposals for similarity have been primarily assessed in terms of how well they can cover Tversky's (1977) key empirical results (Ashby \& Perrin 1988; Krumhansl 1978).

Pothos and Busemeyer (2011) proposed that different concepts in our experience correspond to subspaces of different dimensionality, so that concepts for which there is more extensive knowledge were naturally associated with subspaces of greater dimensionality. Individual dimensions can be broadly understood as concept properties. They suggested that the similarity of a concept $A$ to another concept $B$ (denoted, $\operatorname{Sim}(A, B)$ ) could be modeled with the projection from the subspace for the first concept to the subspace for the second one: $\operatorname{Sim}(A$, $B)=\left\|P_{B} \cdot P_{A} \cdot \Psi\right\|^{2}=\operatorname{Prob}(A \wedge$ then $B)$. Because in QP theory probability is computed from the overlap between a vector and a subspace, it is naturally interpreted as similarity (Sloman 1993). The initial state vector corresponds to whatever a person would be thinking just prior to the 
comparison. This is set so that it is neutral with respect to the $A$ and $B$ subspaces (i.e., prior to the similarity comparison, a participant would not be thinking more about $A$ than about $B$, or vice versa).

Consider one of Tversky's (1977) main findings, that the similarity of Korea to China was judged greater than the similarity of China to Korea (actually, North Korea and communist China; similar asymmetries were reported for other countries). Tversky's proposal was that symmetry is violated, because we have more extensive knowledge about China than about Korea, and, therefore, China has more distinctive features relative to Korea. He was able to describe empirical results with a similarity model based on a differential weighting of the common and distinctive features of Korea and China. However, the only way to specify these weights, was with free parameters and alternative values for the weights, could lead to either no violation of symmetry or a violation in a way opposite to the empirically observed one.

By contrast, using QP theory, if one simply assumes that the dimensionality of the China subspace is greater than the dimensionality of the Korea one, then a violation of symmetry in the required direction readily emerges, without the need for parameter manipulation. As shown in Figure 4, in the Korea to China comparison (4a), the last projection is to a higher dimensionality subspace than is the last projection in the China to Korea comparison (4b). Therefore, in the Korea to China case (4a), more of the amplitude of the original state vector is retained, which leads to a prediction for a higher similarity judgment. This intuition was validated with computational simulations by Pothos and Busemeyer (2011), whose results indicate that, as long as one subspace has a greater dimensionality than another, on average the transition from the lower dimensionality subspace to the higher dimensionality one would retain more amplitude than the converse transition (it has not been proved that this is always the case, but note that participant results with such tasks are not uniform).

\subsection{Other related empirical evidence}

Tversky and Kahneman are perhaps the researchers who most vocally pointed out a disconnect between CP models and cognitive process and, accordingly, we have emphasized QP theory models for some of their most influential findings (and related findings). A skeptical reader may ask, is the applicability of QP theory to cognition mostly restricted to decision making and judgment? Empirical findings that indicate an inconsistency with CP principles are widespread across most areas of cognition. Such findings are perhaps not as well established as the ones reviewed previously, but they do provide encouragement regarding the potential of QP theory in psychology. We have just considered a QP theory model for asymmetries in similarity judgment. Relatedly, Hampton (1988b, Hampton1988 see also Hampton 1988a) reported an overextension effect for category membership. Participants rated the strength of category membership of a particular instance to different categories. For example, the rated membership of "cuckoo" to the pet and bird categories were 0.575 and 1 respectively. However, the corresponding rating for the conjunctive category pet bird was 0.842 , a finding analogous to the conjunction fallacy. This paradigm also produces violations of disjunction. Aerts and Gabora (a)

\section{Korea to China}

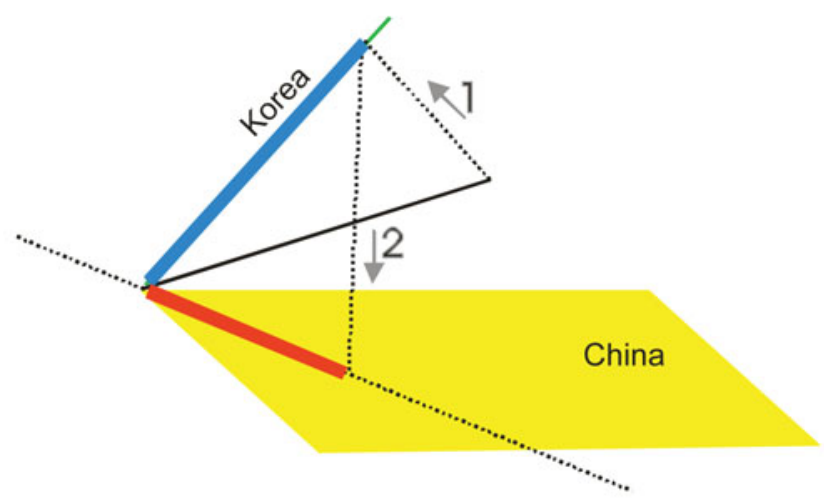

(b)

China to Korea

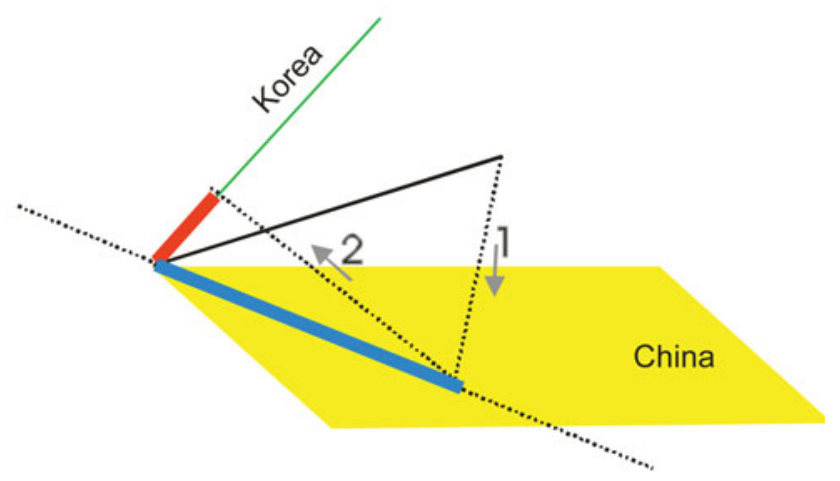

Figure 4. Figure 4a corresponds to the similarity of Korea to China and $4 \mathrm{~b}$ to the similarity of China to Korea. Projecting to a higher dimensionality subspace last (as in $4 \mathrm{a}$ ) retains more of the original amplitude than projecting onto a lower dimensionality subspace last (as in $4 \mathrm{~b}$ ).

(2005b) and Aerts (2009) provided a QP theory account of such findings. Relatedly, Aerts and Sozzo (2011b) examined membership judgments for pairs of concept combinations, and they empirically found extreme forms of dependencies between concept combination pairs, which indicated that it would be impossible to specify a complete joint distribution over all combinations. These results could be predicted by a QP model using entangled states to represent concept pairs.

In memory research, Brainerd and Reyna (2008) discovered an episodic overdistribution effect. In a training part, participants were asked to study a set of items $T$. In test, the training items $T$ were presented together with related new ones, $R$ (and some additional foil items). Two sets of instructions were employed. With the verbatim instructions $(V)$, participants were asked to identify only items from the set $T$. With the gist instructions $(G)$, participants were required to select only $R$ items. In some cases, the instructions (denoted as $V$ or $G$ ) prompted participants to select test items from the $T$ or $R$ sets. From a classical perspective, as a test item comes from either the $T$ set or the $R$ one, but not both, it has to be the case that $\operatorname{Prob}(V \mid T)+\operatorname{Prob}(G \mid T)=$ $\operatorname{Prob}(\operatorname{Vor} G \mid T)$ (these are the probabilities of endorsing a test item from the set $T$, as a function of different instructions). However, Brainerd and Reyna’s (2008) empirical results were inconsistent with the classical prediction. 
Pothos \& Busemeyer: Can quantum probability provide a new direction for cognitive modeling?

Busemeyer and Bruza (2012, Ch. 6) explored in detail a range of models for this memory overdistribution effect (apart from a CP theory model, also a signal detection model, Brainerd et al.'s [1999] dual process model, and a QP theory model). The best performing models were the quantum model and the dual process one, but the ability of the latter to cover empirical results, in this case, perhaps depended too much on an arbitrary bias parameter. Another example from memory research is Bruza et. al.'s (2009) application of quantum entanglement (which implies a kind of holism inconsistent with classical notions of causality) to explain associative memory findings, which cannot be accommodated within the popular theory of spreading activation.

Finally, in perception, Conte et al. (2009) employed a paradigm involving the sequential presentation of two ambiguous figures (each figure could be perceived in two different ways) or the presentation of only one of the figures. It is possible that seeing one figure first may result in some bias in perceiving the second figure. Nonetheless, from a classical perspective, one still expects the law of total probability to be obeyed, so that $p(A+\wedge B-)+p(A+\wedge B+)=p(A+) \quad(A$ and $B$ refer to the two figures and the+and-signs to the two possible ways of perceiving them). It turned out that empirical results were inconsistent with the law of total probability, but a QP model could provide satisfactory coverage. In other perception work, Atmanspacher et al. (2004; Atmanspacher \& Filk 2010) developed and empirically tested a quantum model that could predict the dynamic changes produced during bistable perception. Their model provided a picture of the underlying cognitive process radically different from the classical one. Classically, it has to be assumed that at any given time a bistable stimulus is perceived with a particular interpretation. In Atmanspacher et al.'s (2004) model, by contrast, time periods of perception definiteness were intermixed with periods in which the perceptual impact from the stimulus was described with a superposition state, making it impossible to consider it as conforming to a particular interpretation. Atmanspacher et al.'s (2004) model thus predicted violations of causality in temporal continuity.

\section{General issues for the QP models}

\subsection{Can the psychological relevance of CP theory be disproved?}

It is always possible to augment a model with additional parameters or mechanisms to accommodate problematic results. For example, a classical model could describe the conjunction fallacy in the Linda story by basing judgment not on the difference between a conjunction and an individual probability, but rather on the difference between appropriately set conditional probabilities (e.g., Prob (Linda|bank teller) vs. Prob(Linda|bank teller $\wedge$ feminist); cf. Tenenbaum \& Griffiths 2001). Also, a conjunctive statement can always be conditionalized on presentation order, so that one can incorporate the assumption that the last piece of evidence is weighted more heavily than the first piece. Moreover, deviations from CP predictions in judgment could be explained by introducing assumptions of how participants interpret the likelihood of statements in a particular hypothesis, over and above what is directly stated (e.g., Sher \& McKenzie 2008). Such approaches, however, are often unsatisfactory. Arbitrary interpretations of the relevant probabilistic mechanism are unlikely to generalize to related empirical situations (e.g., disjunction fallacies). Also, the introduction of post-hoc parameters will lead to models that are descriptive and limited in insight. Thus, employing a formal framework in arbitrarily flexible ways to cover problematic findings is possible, but of arguable explanatory value, and it also inevitably leads to criticism (Jones \& Love 2011). But are the findings we considered particularly problematic for CP theory?

$\mathrm{CP}$ theory is a formal framework; that is, a set of interdependent axioms that can be productively employed to lead to new relations. Therefore, when obtaining psychological evidence for a formal framework, we do not just support the particular principles under scrutiny. Rather, such evidence corroborates the psychological relevance of all possible relations that can be derived from the formal framework. For example, one cannot claim that one postulate from a formal framework is psychologically relevant, but another is not, and still maintain the integrity of the theory.

The ingenuity of Tversky, Kahneman, and their collaborators (Kahneman et al. 1982; Shafir \& Tversky 1992; Tversky \& Kahneman 1973) was exactly that they provided empirical tests of principles that are at the heart of CP theory, such as the law of total probability and the relation between conjunction and individual probabilities. Therefore, it is extremely difficult to specify any reasonable $\mathrm{CP}$ model consistent with their results, as such models simply lack the necessary flexibility. There is a clear sense that if one wishes to pursue a formal, probabilistic approach for the Tversky, Kahneman type of findings, then CP theory is not the right choice, even if it is not actually possible to disprove the applicability of $\mathrm{CP}$ theory to such findings.

\subsection{Heuristics vs. formal probabilistic modeling}

The critique of CP theory by Tversky, Kahneman and collaborators can be interpreted in a more general way, as a statement that the attempt to model cognition with any axiomatic set of principles is misguided. These researchers thus motivated their influential program involving heuristics and biases. Many of these proposals sought to relate generic memory or similarity processes to performance in decision making (e.g., the availability and representativeness heuristics; Tversky \& Kahneman 1983). Other researchers have developed heuristics as individual computational rules. For example, Gigerenzer and Todd's (1999) "take the best" heuristic offers a powerful explanation of behavior in a particular class of problem-solving situations.

Heuristics, however well motivated, are typically isolated: confidence in one heuristic does not extend to other heuristics. Therefore, cognitive explanations based on heuristics are markedly different from ones based on a formal axiomatic framework. Theoretical advantages of heuristic models are that individual principles can be examined independently from each other and that no commitment has to be made regarding the overall alignment of cognitive process with the principles of a formal framework. Some theorists would argue that we can only understand cognition through heuristics. However, it is also often the case that heuristics can be re-expressed in a formal way or reinterpreted within CP or QP theory. For example, the heuristics from the Tversky and Kahneman research program, which were developed specifically as an 
Pothos \& Busemeyer: Can quantum probability provide a new direction for cognitive modeling?

alternative to CP models, often invoke similarity or memory processes, which can be related to order/context effects in QP theory. Likewise, failures of consequential reasoning in prisoner's dilemma (Tversky \& Shafir 1992) can be formalized with quantum interference effects.

The contrast between heuristic and formal probabilistic approaches to cognition is a crucial one for psychology. The challenge for advocates of the former is to specify heuristics that cannot be reconciled with formal probability theory (CP or QP). The challenge for advocates of the latter is to show that human cognition is overall aligned with the principles of (classical or quantum) formal theory.

\subsection{Is $Q P$ theory more complex than $C P$ theory?}

We have discussed the features of QP theory, which distinguish it from CP theory. These distinctive features typically emerge when considering incompatible questions. We have also stated that QP theory can behave like CP theory for compatible questions (sect. 2.2.2). Accordingly, there might be a concern that QP theory is basically all of CP theory (for compatible questions) and a bit more, too (for incompatible ones), so that it provides a more successful coverage of human behavior simply because it is more flexible.

This view is incorrect. First, it is true that QP theory for compatible questions behaves a lot like CP theory. For example, for compatible questions, conjunction is commutative, Lüder's law becomes effectively identical to Bayes's law, and no overestimation of conjunction can be predicted. However, CP and QP theories can diverge, even for compatible questions. For example, quantum timedependent models involving compatible questions can still lead to interference effects, which are not possible in classical theory (sect. 2.3). Although CP and QP theories share the key commonality of being formal frameworks for probabilistic inference, they are founded on different axioms and their structure (set theoretic vs. geometric) is fundamentally different. QP theory is subject to several restrictive constraints; however, these are different from the ones in CP theory.

For example, CP Markov models must obey the law of total probability, whereas dynamic QP models can violate this law. However, dynamic QP models must obey the law of double stochasticity, while CP Markov models can violate this law. Double stochasticity is a property of transition matrices that describes the probabilistic changes from an input to an output over time. Markov models require each column of a transition matrix to sum to unity (so that they are stochastic), but QP models require both each row and each column to sum to unity (so they are doubly stochastic). Double stochasticity sometimes fails and this rules out QP models (Busemeyer et al. 2009; Khrennikov 2010).

Moreover, QP models have to obey the restrictive law of reciprocity, for outcomes defined by one-dimensional subspaces. According to the law of reciprocity, the probability of transiting from one vector to another is the same as the probability of transiting from the second vector to the first, so that the corresponding conditional probabilities have to be the same. Wang and Busemeyer (in press) directly tested this axiom, using data on question order, and found that it was upheld with surprisingly high accuracy.
More generally, a fundamental constraint of QP theory concerns Gleason's theorem, namely that probabilities have to be associated with subspaces via the equation

$$
\operatorname{Prob}(A \mid \psi)=\| P_{A}|\psi\rangle \|^{2}
$$

Finding that Gleason's theorem is psychologically implausible would rule out quantum models. A critic may wonder how one could test such general aspects of quantum theory. Recently, however, Atmanspacher and Römer (2012) were able to derive a test for a very general property of QP theory (related to Gleason's theorem). Specifically, they proposed that failures of commutativity between a conjunction and one of the constituent elements of the conjunction (i.e., $A$ vs. $A \wedge B$ ) would preclude a Hilbert space representation for the corresponding problem. These are extremely general predictions and show the principled nature of QP theory approaches to cognitive modeling.

Even if at a broad level CP and QP theories are subject to analogous constraints, a critic may argue that it is still possible that QP models are more flexible (perhaps because of their form). Ultimately, the issue of relative flexibility is a technical one and can only be examined against particular models. So far, there has only been one such examination and, surprisingly, it concluded in favor of QP theory. Busemeyer et al. (2012) compared a quantum model with a traditional decision model (based on prospect theory) for a large data set, from an experiment by Barkan and Busemeyer (2003). The experiment involved choices between gambles, using a procedure similar to that used by Tversky and Shafir (1992) for testing the sure thing principle. The models were equated with respect to the number of free parameters. However, the models could still differ with respect to their complexity. Accordingly, Busemeyer et al. (2012) adopted a Bayesian procedure for model comparison, which evaluates models on the basis of both their accuracy and complexity. As Bayesian comparisons depend upon priors over model parameters, different priors were examined, including uniform and normal priors. For both priors, the Bayes's factor favored the QP model over the traditional model (on average, by a factor of 2.07 for normal priors, and by a factor of 2.47 for uniform priors).

Overall, QP theory does generalize CP theory in certain ways. For example, it allows both for situations that are consistent with commutativity in conjunction (compatible questions) and situations that are not (incompatible questions). However, QP theory is also subject to constraints that do not have an equivalent in $\mathrm{CP}$ theory, such as double stochasticity and reciprocity, and there is currently no evidence that specific QP models are more flexible than $\mathrm{CP}$ ones. The empirical question then becomes: which set of general constraints is more psychologically relevant. We have argued that QP theory is ideally suited for modeling empirical results that depend upon order/context or appear to involve some kind of extreme dependence that rules out classical composition. QP theory was designed by physicists to capture analogous phenomena in the physical world. However, QP theory does not always succeed, and there have been situations in which the assumptions of $\mathrm{CP}$ models are more in line with empirical results (Busemeyer et al. 2006). Moreover, in some situations, the 
Pothos \& Busemeyer: Can quantum probability provide a new direction for cognitive modeling?

predictions from QP and CP models converge, and in such cases it is perhaps easier to employ CP models.

\section{The rational mind}

Beginning with Aristotle and up until recently, scholars have believed that humans are rational because they are capable of reasoning on the basis of logic. First, logic is associated with an abstract elegance and a strong sense of mathematical correctness. Second, logic was the only system for formal reasoning; therefore, scholars could not conceive of the possibility that reasoning could be guided by an alternative system. Logic is exactly this-logical-so how could there be an alternative system for rational reasoning? But this view turned out to be problematic. Considerable evidence accumulated that naive observers do not typically reason with classical logic (Wason 1960); therefore, classical logic could not be maintained as a theory of thinking.

Oaksford and Chater $(2007 ; 2009)$ made a compelling case against the psychological relevance of classical logic. The main problem is that classical logic is deductive, so that once a particular conclusion is reached from a set of premises, this conclusion is certain and cannot be altered by the addition of further premises. Of course, this is rarely true for everyday reasoning. The key aspect of everyday reasoning is its nonmonotonicity, as it is always possible to alter an existing conclusion with new evidence. Oaksford and Chater (2007; 2009) advocated a perspective of Bayesian rationality, which was partly justified using Anderson's (1990) rational analysis approach. According to rational analysis, psychologists should look for the behavior function that is optimal, given the goals of the cognitive agent and its environment. Oaksford and Chater's Bayesian rationality view has been a major contribution to the recent prominence of cognitive theories based on CP theory. For example, CP theories are often partly justified as rational theories of the corresponding cognitive problems, which makes them easier to promote than alternatives. For example, in categorization, the rational model of categorization (e.g., Sanborn et al. 2010) has been called, well, "rational." By contrast, the more successful Generalized Context Model (Nosofsky 1984) has received less corresponding justification (Wills \& Pothos 2012).

There has been considerable theoretical effort to justify the rational status of CP theory. We can summarize the relevant arguments under three headings: Dutch book, long-term convergence, and optimality. The Dutch book argument concerns the long-term consistency of accepting bets. If probabilities are assigned to bets in a way that goes against the principles of CP theory, then this guarantees a net loss (or gain) across time. In other words, probabilistic assignment inconsistent with CP theory leads to unfair bets (de Finetti et al. 1993). Long-term convergence refers to the fact that if the true hypothesis has any degree of non-zero prior probability, then, in the long run, Bayesian inference will allow its identification. Finally, optimality is a key aspect of Anderson's (1990) rational analysis and concerns the accuracy of probabilistic inference. According to advocates of CP theory, this is the optimal way to harness the uncertainty in our environment and make accurate predictions regarding future events and relevant hypotheses.
These justifications are not without problems. Avoiding a Dutch book requires expected value maximization, rather than expected utility maximization, that is, the decision maker is constrained to use objective values rather than personal utilities, when choosing between bets. However, decision theorists generally reject the assumption of objective value maximization and instead allow for subjective utility functions (Savage 1954). This is essential, for example, in order to take into account the observed risk aversion in human decisions (Kahneman \& Tversky 1979). When maximizing subjective expected utility, CP reasoning can fall prey to Dutch book problems (Wakker 2010). Longterm convergence is also problematic, because if the true hypothesis has a prior probability of zero, it can never be identified. This is invariably the case in Bayesian models, as it is not possible to assign a non-zero probability to all candidate hypotheses. Overall, a priori arguments, such as the Dutch book or long-term convergence, are perhaps appealing under simple, idealized conditions. However, as soon as one starts taking into account the complexity of human cognition, such arguments break down.

Perhaps the most significant a priori justification for the rationality of $\mathrm{CP}$ theory concerns optimality of predictions. If reasoning on the basis of CP theory is optimal, in the sense of predictive accuracy, then this seems to settle the case in favor of CP theory. For example, is it more accurate to consider Linda as just a bank teller, rather than as a bank teller and a feminist? By contrast, QP theory embodies a format for probabilistic inference which is strongly perspective and context dependent. For example, Linda may not seem like a bank teller initially, but from the perspective of feminism such a property becomes more plausible. However, optimality must be evaluated under the constraints and limited resources of the cognitive system (Simon 1955).

The main problem with classical optimality is that it assumes a measurable, objective reality and an omniscient observer. Our cognitive systems face the problem of making predictions for a vast number of variables that can take on a wide variety of values. For the cognitive agent to take advantage of classical optimality, it would have to construct an extremely large joint probability distribution to represent all these variables (this is the principle of unicity). But for complex possibilities, it is unclear as to where such information would come from. For example, in Tversky and Kahneman's (1983) experiment we are told about Linda, a person we have never heard of before. Classical theory would assume that this story generates a sample space for all possible characteristic combinations for Linda, including unfamiliar ones such as feminist bank teller. This just doesn't seem plausible, let alone practical, considering that for the bulk of available knowledge, we have no relevant experience. It is worth noting that Kolmogorov understood this limitation of CP theory (Busemeyer \& Bruza 2012, Ch. 12). He pointed out that his axioms apply to a sample space from a single experiment and that different experiments require new sample spaces. But his admonitions were not formalized, and CP modelers do not take them into account.

Quantum theory assumes no measurable objective reality; rather judgment depends on context and perspective. The same predicate (e.g., that Linda is a bank teller) may appear plausible or not, depending upon the point of view (e.g., depending on whether we accept Linda as a feminist or not). Note that QP theory does assume 
systematic relations between different aspects of our knowledge, in terms of the angle (and relative dimensionality) between different subspaces. However, each inference changes the state vector, and, therefore, the perspective from which all other outcomes can be evaluated. Note also that context effects in QP theory are very different from conditional probabilities in CP theory. The latter are still assessed against a common sample space. With the former, the sample space for a set of incompatible outcomes changes every time an incompatible question is evaluated (as this changes the basis for evaluating the state).

If we cannot assume an objective reality and an omniscient cognitive agent, then perhaps the perspective-driven probabilistic evaluation in quantum theory is the best practical rational scheme. In other words, quantum inference is optimal, for when it is impossible to assign probabilities to all relevant possibilities and combinations concurrently. This conclusion resonates with Simon's (1955) influential idea of bounded rationality, according to which cognitive theory needs to incorporate assumptions about the computational burden which can be supported by the human brain. For example, classically, the problem of assessing whether Linda is a feminist and a bank teller requires the construction of a bivariate joint probability space, which assigns a probability density for each outcome regarding these questions. By contrast, a QP representation is simpler: it requires a univariate amplitude distribution for each question, and the two distributions can be related through a rotation. As additional questions are considered (e.g., whether Linda might be tall or short) the efficiency of the QP representation becomes more pronounced. Note that classical schemes could be simplified by assuming independence between particular outcomes. However, independence assumptions are not appropriate for many practical situations and will introduce errors in inference.

Note that the perspective dependence of probabilistic assessment in QP theory may seem to go against an intuition that "objective" (classical) probabilities are somehow more valid or correct. However, this same probabilistic scheme does lead to more accurate predictions in the physical world, in the context of quantum physics. If the physical world is not "objective" enough for CP theory to be used, there is a strong expectation that the mental world, with its qualities of flux and interdependence of thoughts, would not be also.

The application of QP theory to cognition implies a strong interdependence between thoughts, such that it is typically not possible to have one thought without repercussions for other thoughts. These intuitions were extensively elaborated in the work of Fodor (1983), with his proposals that thought is isotropic and Quinean, so that revising or introducing one piece of information can in principle impact on most other information in our knowledge base. Oaksford and Chater (2007; 2009) argued that it is exactly such characteristics of thought that make CP theory preferable to classical logic for cognitive modeling. However, Fodor's (1983) arguments also seem to go against the neat reductionism in $\mathrm{CP}$ theory, required by the principle of unicity and the law of total probability, according to which individual thoughts can be isolated from other, independent ones, and the degree of interdependence is moderated by the requirement to always have a joint probability between all possibilities. QP theory is not subject to these constraints.
Overall, accepting a view of rationality inconsistent with classical logic was a major achievement accomplished by CP researchers (e.g., Oaksford \& Chater 2007; 2009). For example, how can it be that in the Wason selection task the "falsificationist" card choices are not the best ones? Likewise, accepting a view of rationality at odds with $\mathrm{CP}$ theory is the corresponding challenge for $\mathrm{QP}$ researchers. For example, how could it not be that Prob $(A \wedge B)=\operatorname{Prob}(B \wedge A)$ ? The principles of CP theory have been accepted for so long that they are considered self evident. However, one of our objectives in Section 3 was exactly to show how QP theory can lead to alternative, powerful intuitions about inference, intuitions that emphasize the perspective-dependence of any probabilistic conclusion. We conclude with an interesting analogy. Classical logic can be seen as a rational way of thinking, but only in idealized situations in which deductive inference is possible, that is, such that there are no violations of monotonicity. CP theory inference can also be seen as rational, but only in idealized situations in which the requirements from the principle of unicity match the capabilities of the observers (i.e., the possibilities that require probabilistic characterization are sufficiently limited). For the real, noisy, confusing, ever-changing, chaotic world, QP is the only system that works in physics and, we strongly suspect, in psychology as well.

\section{Concluding comments}

\subsection{Theoretical challenges}

The results of Tversky, Kahneman, and colleagues (e.g., Tversky \& Kahneman 1974) preclude a complete explanation of cognitive processes with CP theory. We have suggested that QP theory is the appropriate framework to employ for cases in which CP theory fails. QP and CP theories are closely related and, also, the kind of models produced from CP and QP theories are analogous. Therefore, it could be proposed that using CP and QP theories together, a complete explanation of cognitive processes would emerge.

In exploring such a proposal, the first step should be to identify the precise boundary conditions between the applicability and failure of CP principles in cognitive modeling. In other words, there is no doubt that in some cases cognitive process does rely on CP principles (perhaps the same can also be said for classical logic principles). The results of Tversky et al. (1974) reveal situations in which this reliance breaks down. A key theoretical challenge concerns understanding the commonalities between the experimental situations that lead to failures in the applicability of classical principles. For example, what exactly triggers the relevance of a quantum probabilistic reasoning approach in situations as diverse as the Linda problem, violations of the sure thing principle in prisoner's dilemma, and sets of questions concerning United States presidential candidates? A preliminary suggestion is that perhaps the more idealized the reasoning situation (e.g., is it cognitively feasible to apply the unicity principle?), the greater the psychological relevance of CP theory.

Another challenge concerns further understanding the rational properties of quantum inference. The discussion in Section 5 focused on the issue of accuracy, assuming that the requirements from the principle of unicity have 
Pothos \& Busemeyer: Can quantum probability provide a new direction for cognitive modeling?

to be relaxed. However, there is a further, potentially relevant literature on quantum information theory (Nielsen \& Chuang 2000), which concerns the processing advantages of probabilistic inference based on QP theory. For example, a famous result by Grover (1997) shows how a quantum search algorithm will outperform any classical algorithm. The potential psychological relevance of such results (e.g., in categorization theory) is an issue for much further work (e.g., is it possible to approximate quantum information algorithms in the brain?). These are exciting possibilities regarding both the rational basis of quantum cognitive models and the general applicability of quantum theory to cognitive theory.

\subsection{Empirical challenges}

So far, the quantum program has involved employing quantum computational principles to explain certain, prominent empirical findings. Such quantum models do not simply - redescribe of results that have already had (some) compelling explanation. Rather, we discussed results that have presented ongoing challenges and have resisted explanation based on classical principles. One objective for future work is to continue identifying empirical situations that are problematic from a classical perspective.

Another objective is to look for new, surprising predictions, which take advantage of the unique properties of quantum theory, such as superposition, incompatibility, and entanglement. For example, Trueblood and Busemeyer (2011) developed a model to accommodate order effects in the assessment of evidence in McKenzie et al.'s (2002) task. The model successfully described data from both the original conditions and a series of relevant extensions. Moreover, Wang and Busemeyer (in press) identified several types of order effects that can occur in questionnaires, such as consistency and contrast (Moore 2002). Their quantum model was able to make quantitative, parameter-free predictions for these order effects. In perception, Atmanspacher and Filk (2010) proposed an experimental paradigm for bistable perception, so as to test the predictions from their quantum model regarding violations of the temporal Bell inequality (such violations are tests of the existence of superposition states).

Overall, understanding the quantum formalism to the extent that surprising, novel predictions for cognition can be generated is no simple task (in physics, this was a process that took several decades). The current encouraging results are a source of optimism.

\subsection{Implications for brain neurophysiology}

An unresolved issue is how QP computations are implemented in the brain. We have avoided a detailed discussion of this research area because, although exciting, is still in its infancy. One perspective is that the brain does not instantiate any quantum computation at all. Rather, interference effects in the brain can occur if neuronal membrane potentials have wave-like properties, a view that has been supported in terms of the characteristics of electroencephalographic (EEG) signals (de Barros \& Suppes 2009). Relatedly, Ricciardi and Umezawa (1967), Jibu \& Yasue (1995), and Vitiello (1995) developed a quantum field theory model of human memory, which still allows a classical description of brain activity. The most controversial
(Atmanspacher 2004; Litt et al. 2006) perspective is that the brain directly supports quantum computations. For quantum computation to occur, a system must be isolated from the environment, as environmental interactions cause quantum superposition states to rapidly decohere into classical states. Penrose (1989) and Hammeroff (1998) suggested that microtubules prevent decoherence for periods of time long enough to enable meaningful quantum computation; in this view, the collapse of superposition states is associated with experiences of consciousness.

Overall, in cognitive science it has been standard to initially focus on identifying the mathematical principles underlying cognition, and later address the issue of how the brain can support the postulated computations. However, researchers have been increasingly seeking bridges between computational and neuroscience models. Regarding the QP cognitive program, this is clearly an important direction for future research.

\subsection{The future of QP theory in psychology}

There is little doubt that extensive further work is essential before all aspects of QP theory can acquire psychological meaning. But this does not imply that current QP models are not satisfactory. In fact, we argue that the quantum approach to cognition embodies all the characteristics of good cognitive theory: it is based on a coherent set of formal principles, the formal principles are related to specific assumptions about psychological process (e.g., the existence of order/context effects in judgment), and it leads to quantitative computational models that can parsimoniously account for both old and new empirical data. The form of quantum cognitive theories is very much like that of CP ones, and the latter have been hugely influential in recent cognitive science. The purpose of this article is to argue that researchers attracted to probabilistic cognitive models need not be restricted to classical theory. Rather, quantum theory provides many theoretical and practical advantages, and its applicability to psychological explanation should be further considered.

\section{APPENDIX}

An elaboration of some of the basic definitions in QP theory

(See Busemeyer \& Bruza 2012 for an extensive introduction)

\section{Projectors (or projection operators)}

Projectors are idempotent linear operators. For a onedimensional subspace, corresponding, for example, to the $|h a p p y\rangle$ ray, the projector is a simple outer product, $P_{\text {happy }}=\mid$ happy $\rangle\langle$ happy $|$. Note that $\mid$ happy $\rangle$ corresponds to a column vector and $\langle$ happy| denotes the corresponding row vector (with conjugation).

Given the above subspace for "happy," the probability that a person is happy is given by

$$
\left.\| P_{\text {happy }}|\psi\rangle\left\|^{2}=\right\| \text { happy }\right\rangle\langle\text { happy } \mid \psi\rangle \|^{2} .
$$

In this expression, $\langle$ happy $\mid \Psi\rangle$ is the standard dot (inner) product and $|h a p p y\rangle$ is a unit length vector. Therefore,

$$
\| P_{\text {happy }}|\psi\rangle \|^{2}=\mid\left.\langle h \text { appy } \mid \psi\rangle\right|^{2} .
$$


Pothos \& Busemeyer: Can quantum probability provide a new direction for cognitive modeling?

The double lines on the left hand side of this equation denote the length of the vector, so that $\| P_{\text {happy }}|\Psi\rangle \|^{2}$ is the length of the vector squared. The single lines on the right hand side denote the modulus (magnitude) of a complex number. Therefore, in a real space, we can simply write

$$
\| P_{h a p p y}|\psi\rangle \|^{2}=(\langle h a p p y \mid \psi\rangle)^{2}
$$

\section{Composite systems}

Two subspaces can be combined into a composite space in two ways: one way is by forming a tensor product space (as in Figure 1b) and the other way is by forming a space from a direct sum. First consider the formation of a tensor product space. For example, suppose |happy $\rangle$, | happy $\rangle$ are two basis vectors that span the subspace $H$, representing the possibility of happiness, and suppose $|e m p l o y e d\rangle$, $\mid \sim$ employed $\rangle$ are two basis vectors that span the subspace $E$, representing the possibility of employment. Then, the tensor product space equals the span of the four basis vectors formed by the tensor products

$$
\begin{aligned}
& \{\mid \text { happy }\rangle \otimes \mid \text { employed }\rangle, \mid \text { happy }\rangle \otimes \mid \sim \text { employed }\rangle, \\
& \mid \sim \text { happy }\rangle \otimes \mid \text { employed }\rangle, \mid \sim \text { happy }\rangle \otimes \mid \sim \text { employed }\rangle\} .
\end{aligned}
$$

Next consider the formation of a space by direct sum. For example, suppose the subspace $E$ is spanned by the basis vectors

$$
\{\mid \text { happy }\rangle \otimes \mid \text { employed }\rangle, \mid \sim \text { happy }\rangle \otimes \mid \text { employed }\rangle\}
$$

and suppose $\sim \mathrm{E}$ is the subspace spanned by the basis vectors

$$
\{\mid \text { happy }\rangle \otimes \mid \sim \text { employed }\rangle, \mid \sim \text { happy }\rangle \otimes \mid \sim \text { employed }\rangle\} .
$$

Then the direct sum space is formed by all possible pairs of vectors, one from $E$ and another from $\sim E$.

\section{Time dependence}

The quantum state vector changes over time according to Schrödinger's equation,

$$
\frac{d}{d t}|\psi(t)\rangle=-i \cdot H \cdot|\psi(t)\rangle
$$

where $H$ is a Hermitian linear operator. This is the QP theory equivalent of the Kolmogorov forward equation for Markov models in CP theory. The solution to Schrödinger's equation equals

$$
\psi_{2}(t)=e^{-i \cdot t \cdot H} \cdot \psi_{1}=U(t) \cdot \psi_{1}
$$

where $H$ is a Hermitian operator and $U(t)$ is a unitary one (note that $i^{2=}-1$ ). The two (obviously related) operators $H$ and $U(t)$ contain all the information about the dynamical aspects of a system. The key property of $U(t)$ is that it preserves lengths, so that
Thus, the effect of $U(t)$ on a state vector is to rotate it in a way that captures some dynamical aspect of the situation of interest.

\section{An example of how interference can arise in QP theory}

Consider a situation whereby a person tries to assess whether she is happy or not, depending upon whether she is employed or not. We can write

$$
\mid \text { Initial state }\rangle=\frac{1}{\sqrt{2}}\left|\psi_{\text {employed }}\right\rangle+\frac{1}{\sqrt{2}}\left|\psi_{\sim_{\text {employed }}}\right\rangle
$$

(this corresponds to a direct sum decomposition). Assume that this initial state develops in time with a unitary matrix, $U(t)$, which could correspond to the thought process of weighting the implications of being and not being employed for happiness (Pothos \& Busemeyer 2009; Trueblood \& Busemeyer 2011), so we end up with

$$
\mid \text { final state }\rangle=U(t) \cdot \frac{1}{\sqrt{2}}\left|\psi_{\text {employed }}\right\rangle+U(t) \cdot \frac{1}{\sqrt{2}}\left|\psi_{\sim_{\text {employed }}}\right\rangle .
$$

Note that so far the situation is identical to what we would have had if we were applying a CP theory Markov model.

The difference between the Markov CP model and the QP one is in how probabilities are computed from the time-evolved state. Consider the probability for being happy; this can be extracted from a state by applying a projector operator $M$ that picks out the coordinates for being happy. In the QP case,

$$
\begin{aligned}
& \operatorname{Prob} \text { (happy, unknown employment) } \\
& \quad=\| M \cdot U(t) \cdot \frac{1}{\sqrt{2}}\left|\psi_{\text {employed }}\right\rangle+M \cdot U(t) \cdot \frac{1}{\sqrt{2}}\left|\psi_{\sim_{\text {employed }}}\right\rangle \|^{2}
\end{aligned}
$$

That is, as has been discussed, probabilities are computed from amplitudes through a squaring operation. This nonlinearity in QP theory can lead to interference terms that produce violations of the law of total probability. Specifically,

$$
\begin{aligned}
& \text { Prob(happy, unknown employment }) \\
& =\text { Prob(happy } \wedge \text { employed })+\operatorname{Prob}(\text { happy } \wedge \sim \text { employed }) \\
& \quad+\text { Interference terms. }
\end{aligned}
$$

As the interference terms can be negative, the law of total probability can be violated.

Suppose next that the person is determined to find out whether she will be employed or not, before having this inner reflection about happiness (perhaps she intends to delay thinking about happiness until after her professional review). Then, the state after learning about her employment will be either $\left|\Psi_{\text {employed }}\right\rangle$ or $\left|\Psi_{\sim_{\text {employed }}}\right\rangle$. Therefore, what will evolve is one of these two states. Therefore, for example,

$$
\left.\operatorname{Prob}(\text { happy } \mid \text { employed })=|M \cdot U(t) \cdot| \psi_{\text {employed }}\right\rangle\left.\right|^{2}
$$

and
$\operatorname{Prob}($ happy $\mid \sim$ employed $\left.)=|M \cdot U(t) \cdot| \psi \sim_{\text {employed }}\right\rangle\left.\right|^{2}$. 
Commentary/Pothos \& Busemeyer: Can quantum probability provide a new direction for cognitive modeling?

Overall, in this case,

$$
\begin{aligned}
& \operatorname{Prob}(\text { happy, unknown employment }) \\
& \begin{aligned}
\left.=|M \cdot U(t) \cdot| \psi_{\text {employed }}\right\rangle\left.\right|^{2} \cdot \operatorname{Prob}(\text { employed }) \\
\left.\quad+|M \cdot U(t) \cdot| \psi \sim_{\text {employed }}\right\rangle\left.\right|^{2} \cdot \operatorname{Prob}(\sim \text { employed }) .
\end{aligned}
\end{aligned}
$$

It should be clear that in such a case there are no interference terms and the quantum result converges to the classical one. Note that the "quantum" reasoner is still uncertain about whether she will be employed. The crucial difference is that in this case she knows she will resolve the uncertainty regarding employment, before her inner reflection. Therefore, regardless of the outcome regarding employment, the evolved state will be a state that is not a superposition one.

\section{ACKNOWLEDGMENTS}

We are grateful to Diederik Aerts, Harald Atmanspacher, Thomas Filk, James Hampton, Mike Oaksford, Steven Sloman, Jennifer Trueblood, and Christoph Weidemann for their helpful comments. Jerome Busemeyer was supported by the grant NSF ECCS-1002188.

\section{Open Peer Commentary}

\section{Quantum structure and human thought}

\author{
doi:10.1017/S0140525X12002841
}

\author{
Diederik Aerts, ${ }^{a}$ Jan Broekaert, ${ }^{a}$ Liane Gabora, ${ }^{\mathrm{b}}$ and \\ Sandro Sozzo ${ }^{a}$ \\ ${ }^{\mathrm{a}}$ Center Leo Apostel, Departments of Mathematics and Psychology, Brussels \\ Free University, 1050 Brussels, Belgium; ${ }^{\mathrm{b}}$ Department of Psychology, \\ University of British Columbia, Okanagan Campus, Kelowna, BC V1V 1V7, \\ Canada. \\ diraerts@vub.ac.be \\ http://www.vub.ac.be/CLEA/aerts \\ jbroekae@vub.ac.be \\ http://www.vub.ac.be/CLEA/Broekaert/ \\ liane.gabora@ubc.ca \\ http://www.vub.ac.be/CLEA/liane \\ ssozzo@vub.ac.be \\ http://www.vub.ac.be/CLEA/people/sozzo/
}

\begin{abstract}
We support the authors' claims, except that we point out that also quantum structure different from quantum probability abundantly plays a role in human cognition. We put forward several elements to illustrate our point, mentioning entanglement, contextuality, interference, and emergence as effects, and states, observables, complex numbers, and Fock space as specific mathematical structures.
\end{abstract}

The authors convincingly demonstrate the greater potential of quantum probability as compared with classical probability in modeling situations of human cognition, giving various examples to illustrate their analysis. In our commentary, we provide additional arguments to support their claim and approach. We want to point out, however, that it is not just quantum probability, but also much more specific quantum structures, quantum states, observables, complex numbers, and typical quantum spaces - for example, Fock space - that on a deep level provide a modeling of the structure of human thought itself.
A first insight about quantum structure in human cognition came to us with the characterizations of classical and quantum probability following from the hidden-variable investigation in quantum theory - that is, the question of whether classical probability can model the experimental data of quantum theory (Bell 1964; Einstein et al. 1935). From these investigations, it follows that when probability is applied generally to a physical system, classical probability models the lack of knowledge of an underlying deterministic reality, whereas non-classical probability, and possibly quantum probability, results when indeterminism arises from the interaction between (measurement) context and system, introducing genuine potentiality for the system states (Aerts 1986). This allowed the identification of situations in macroscopic reality entailing such non-classical indeterminism and therefore being unable to be modeled by classical probability (Aerts 1986; Aerts et al. 1993). It shows that opinion polls, where human decisions are intrinsically influenced by the context, constitute such situations, and therefore entail non-classical probability (Aerts \& Aerts 1995). Our first argument to support and strengthen the authors' claim is that a generalization of classical probability is necessary whenever intrinsically contextual situations evoking indeterminism and potentiality are present (Aerts 1986). We believe this to be commonly the case in situations of human cognition, and believe quantum probability to be a plausible description for this indeterminism and potentiality.

Another result followed from studying the structure and dynamics of human concepts themselves: how concepts combine to form sentences and carry meaning in human thought. An investigation into the relation of concepts to their exemplars allowed for the devising of a Gedankenexperiment violating Bell's inequalities, identifying the presence of quantum entanglement (Aerts et al. 2000). Considering a combination of concepts and its relation to exemplars led to an experimental violation of Bell's inequalities, proving that concepts entangle when they combine (Aerts \& Sozzo 2011a). We studied the guppy effect: Whereas a guppy is considered a very typical "pet-fish," it is regarded as neither a typical "pet" nor a typical "fish." The study of this effect, proved to be impossible to model with classical fuzzy sets by Osherson and Smith (1981), led us to develop a quantum-based concept theory presenting the guppy effect as a non-classical context influence. Concepts are modeled as entities in states in a complex Hilbert space, and quantities such as typicality are described by observables in the quantum formalism (Aerts \& Gabora 2005a; 2005b; Gabora \& Aerts 2002). Our second argument to support and strengthen the authors' approach is that next to quantum effects such as entanglement and contextuality, typical quantum representations of states and observables appear in the combination dynamics of human concepts.

An abundance of experimental data violating set theoretical and classical logic relations in the study of the conjunctions and disjunctions of concepts (Hampton 1988b; 1998b) led to the identification of new quantum effects-interference and emergence-when these data were modeled using our quantum concept formalism. Fock space, a special Hilbert space also used in quantum field theory, turns out to constitute a natural environment for these data. For the combination of two concepts, the first sector of Fock space, mathematically describing interference, corresponds to emergent aspects of human thought, and the second sector, mathematically describing entanglement, corresponds to logical aspects of human thought (Aerts 2007; 2009; 2011; Aerts \& D’Hooghe 2009; Aerts et al., in press). The quantum superposition in Fock space, representing both emergent and logical thought, models Hampton's (1988a; 1988b) data well in our approach. Our third argument to support and strengthen the authors analysis is that the quantum formalism, and many detailed elements of its mathematical structure, for example, Fock space, has proved to be relevant for the structure of human thought itself. 
Commentary/Pothos \& Busemeyer: Can quantum probability provide a new direction for cognitive modeling?

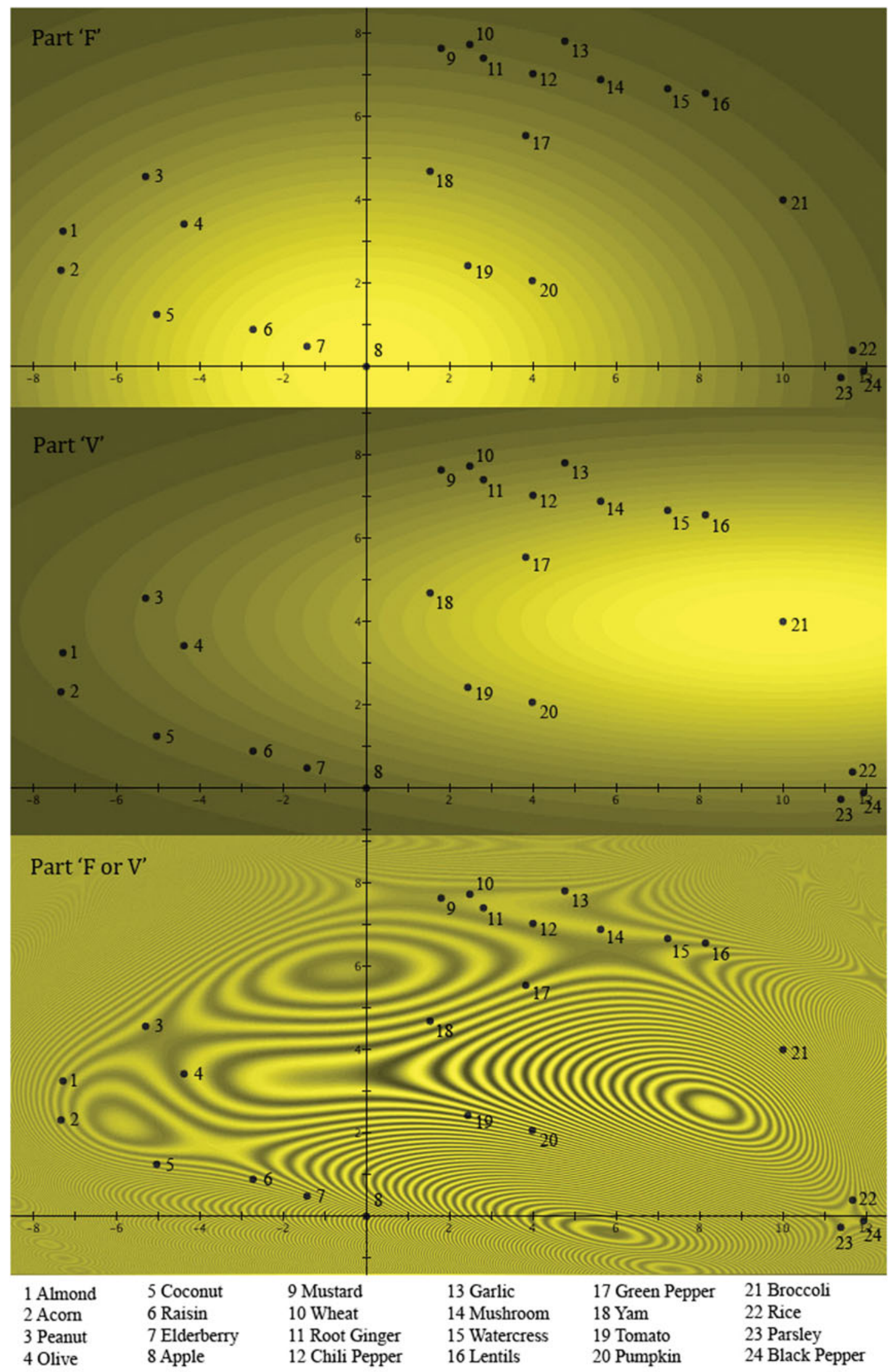

Figure 1 (Aerts et al.). Part "F," Part "V," and Part "F or V" are a graphical representation of the relative membership weights of the indicated exemplars with respect to "fruits," "vegetables," and "fruits or vegetables," respectively. The light intensity at the spots where the exemplars are located is a measure of the relative membership weight at that spot, and hence the graphs can be interpreted as light sources passing through slits "F," "V," and "F and V." 
Commentary/Pothos \& Busemeyer: Can quantum probability provide a new direction for cognitive modeling?

Table 1 (Aerts et al.). Relative membership weights of exemplars with respect to fruits, vegetables and fruits or vegetables as measured by Hampton (1988a)

\begin{tabular}{|c|c|c|c|c|}
\hline & & Fruits & Vegetables & $\begin{array}{l}\text { Fruits or } \\
\text { vegetables }\end{array}$ \\
\hline 1 & Almond & 0.0359 & 0.0133 & 0.0269 \\
\hline 2 & Acorn & 0.0425 & 0.0108 & 0.0249 \\
\hline 3 & Peanut & 0.0372 & 0.0220 & 0.0269 \\
\hline 4 & Olive & 0.0586 & 0.0269 & 0.0415 \\
\hline 5 & Coconut & 0.0755 & 0.0125 & 0.0604 \\
\hline 6 & Raisin & 0.1026 & 0.0170 & 0.0555 \\
\hline 7 & Elderberry & 0.1138 & 0.0170 & 0.0480 \\
\hline 8 & Apple & 0.1184 & 0.0688 & 0.0670 \\
\hline 9 & Mustard & 0.0149 & 0.0250 & 0.0146 \\
\hline 10 & Wheat & 0.0138 & 0.0255 & 0.0165 \\
\hline 11 & Root ginger & 0.0157 & 0.0323 & 0.0385 \\
\hline 12 & $\begin{array}{l}\text { Chili } \\
\text { pepper }\end{array}$ & 0.0167 & 0.0446 & 0.0323 \\
\hline 13 & Garlic & 0.0100 & 0.0301 & 0.0293 \\
\hline 14 & Mushroom & 0.0140 & 0.0545 & 0.0604 \\
\hline 15 & Watercress & 0.0112 & 0.0658 & 0.0482 \\
\hline 16 & Lentils & 0.0095 & 0.0713 & 0.0338 \\
\hline 17 & $\begin{array}{l}\text { Green } \\
\text { pepper }\end{array}$ & 0.0324 & 0.0788 & 0.0506 \\
\hline 18 & Yam & 0.0533 & 0.0724 & 0.0541 \\
\hline 19 & Tomato & 0.0881 & 0.0679 & 0.0688 \\
\hline 20 & Pumpkin & 0.0797 & 0.0713 & 0.0579 \\
\hline 21 & Broccoli & 0.0143 & 0.1284 & 0.0642 \\
\hline 22 & Rice & 0.0140 & 0.0412 & 0.0248 \\
\hline 23 & Parsley & 0.0155 & 0.0266 & 0.0308 \\
\hline 24 & $\begin{array}{l}\text { Black } \\
\text { pepper }\end{array}$ & 0.0127 & 0.0294 & 0.0222 \\
\hline
\end{tabular}

We finish our commentary by presenting a graphic illustration of the interference of concepts as it appears in our quantum concept theory. Figure 1 represents the cognitive interference of the two concepts "fruits" and "vegetables" combined in the disjunction "fruits or vegetables." Part "F," Part "V," and Part "F or $V$ " illustrate the relative membership weights of the different exemplars with respect to "fruits," "vegetables," and "fruits or vegetables," respectively, measured in Hampton (1988a) and presented in Table 1. The illustration is built following standard quantum theory in a Hilbert space of complex wave functions in a plane. The exemplars are located at spots of the plane such that the squares of the absolute values of the quantum wave functions for "fruits," "vegetables" and "fruits or vegetables" coincide with the relative membership weights measured.

The wave function for "fruits or vegetables" is the normalized sum - that is, the superposition - of the two wave functions for "fruits" and for "vegetables," and hence the square of its absolute value includes an interference term. The light intensity at the spots where the exemplars are located is a measure of the relative membership weight at that spot, which means that the graphs can be seen as representations of light passing through slits, where Part "F" corresponds to slit "F" open, Part "V" to slit "V" open, and Part "F or V" to both slits "F" and "V" open. Hence, the graphs illustrate the cognitive interference of "fruits or vegetables" in analogy with the famous double-slit interference pattern of quantum theory (Feynman 1988). The interference pattern is clearly visible (Part "F or V" of Fig. 1), and very similar to well-known interference patterns of light passing through an elastic material under stress. Mathematical details can be found in Aerts et al. (in press).

\section{At home in the quantum world}

\section{doi:10.1017/S0140525X12002853}

\section{Harald Atmanspacher \\ Institute for Frontier Areas of Psychology, D-79098 Freiburg, Germany; Collegium Helveticum, $\mathrm{CH}-8092$ Zurich, Switzerland. atmanspacher@collegium.ethz.ch http://www.igpp.de/english/tda/cv/cv_ha.htm}

Abstract: One among many misleading quotations about the alleged mysteries of quantum theory is from Feynman (1965): "I think I can safely say that nobody understands quantum mechanics." Today we know that quantum theory describes many aspects of our world in a fully intelligible fashion. Pothos \& Busemeyer (P\&B) propose ways in which this may include psychology and cognitive science.

It was an old idea by Niels Bohr, one of the founding architects of quantum physics, that central features of quantum theory, such as complementarity, are also of pivotal significance beyond the domain of physics. Bohr became familiar with the notion of complementarity through the psychologist Edgar Rubin and, indirectly, William James (Holton 1970). Although Bohr always insisted on the extraphysical relevance of complementarity, he never elaborated this idea in concrete detail, and for a long time no one else did so either. This situation has changed; there are now a number of research programs applying key notions of quantum theory beyond physics, in particular to psychology and cognitive science.

The first steps in this direction were made by Aerts and collaborators in the early 1990s (Aerts \& Aerts 1995) in the framework of non-Boolean logic of incompatible (complementary) propositions. Alternative ideas come from Khrennikov (1999), focusing on nonclassical probabilities, and Atmanspacher et al. (2002), proposing an algebraic framework with non-commuting operations. More recently, Bruza and colleagues as well as Busemeyer and colleagues have moved this novel field of research even more into the center of attention. The target article by Pothos \& Busemeyer (P\&B), and a novel monograph by Busemeyer and Bruza (2012), reflect these developments.

Intuitively, it is plausible that non-commuting operations or nonBoolean logic should be relevant, even inevitable, for mental systems. The non-commutativity of operations simply means that the sequence in which operations are applied matters for the final result. This is so if individual mental states are assumed to be dispersive (as individual quantum states are, as opposed to classical states). As a consequence, their observation amounts not only to registering a value, but entails a backreaction changing the observed state: something that seems evident for mental systems.

Non-Boolean logic refers to propositions that may have unsharp truth values beyond "yes" or "no." However, this is not the result of subjective ignorance but must be understood as an intrinsic feature. The proper framework for a logic of incompatible propositions is a partial Boolean lattice (Primas 2007), where locally Boolean sublattices are pasted together in a globally non-Boolean way-just like an algebra of generally non-commuting operations may contain a subset of commuting operations.

Although these formal extensions are essential for quantum theory, they have no dramatic effect on the way in which experiments are evaluated. The reason is that the measuring tools, even in quantum physics, are typically Boolean filters, and, therefore, virtually all textbooks of quantum physics get along with standard probability theory à la Kolmogorov. Only if incompatible experimental scenarios are to be comprehensively discussed in one single picture do the pecularities provided by non-classical thinking become evident and force us to leave outmoded classical reasoning.

In this sense, the authors use the notion of "quantum probability" for psychological and cognitive models and their predictions (cf. Gudder 1988; Redei \& Summers 2007). As Busemeyer points out, an experiment in psychology is defined as a collection of experimental conditions. Each one of them produces indivisible outcomes 
Commentary/Pothos \& Busemeyer: Can quantum probability provide a new direction for cognitive modeling?

forming a complete set of mutually exclusive events. Whereas Kolmogorov probabilities refer to events for a single condition, quantum probabilities refer to the entire set of incompatible conditions, necessary for a comprehensive description of the experiment.

In such a description, events are represented as subspaces of a Hilbert space (as in quantum physics), and all subspaces correspond to orthogonal projectors. A state is defined as a vector, and the probability of an event equals the squared length of the projection of the state onto the subspace representing that event. As all events under each single experimental condition commute, they form a Boolean algebra and the probabilities assigned to them satisfy the axiomatics of Kolmogorov. However, all events of the entire experiment (i.e., the events of all experimental conditions) only form a partial Boolean algebra if some of them do not commute. And as Kolmogorov's axioms imply Bayes' rule, Bayesian reasoning, very influential in psychology, will generally fail to describe experiments with incompatible conditions properly.

Whereas the authors focus on decision theory, routes to be explored in more detail include uncertainty relations, in which order effects arise in variances in addition to mean shifts (Atmanspacher \& Römer 2012). A key feature of quantum theory, entanglement as tested by Bell-type inequalities, has been suggested by Atmanspacher and Filk (2010) for bistable perception and by Bruza et al. (2012) for non-decomposable concept combinations.

Another possible move to incorporate complementarity and entanglement in psychology is based on a state space description of mental systems. If mental states are defined on the basis of cells of a state space partition, then this partition needs to be well tailored to lead to robustly defined states. Ad hoc chosen partitions will generally create incompatible descriptions (Atmanspacher \& beim Graben 2007) and states may become entangled (beim Graben et al. 2013). This way it is possible to understand why mental activity may exhibit features of quantum behavior whereas the underlying neural dynamics are strictly classical.

A further important issue is the complexity or parsimony of Hilbert space models as compared with classical (Bayesian, Markov) models. Atmanspacher and Römer (2012) proposed an option to test limitations of Hilbert space modeling by outcomes of particular joint measurements. Such tests presuppose that the situation under study is framed well enough to enable welldefined research questions; a requirement that must be carefully observed to avoid superficial reasoning without sustainable substance.

With the necessary caution, I am optimistic that this novel field will grow from work in progress to an important subject area of psychology. A quantum theoretically inspired understanding of reality, including cognition, will force us to revise plugged-in cliches of thinking and resist overly naive world views. The Boolean "either-or" in logic and the law of commutativity in elementary calculations are special cases with their own significance, but it would be wrong to think that their generalization holds potential only for exotic particles and fields in physics. The opposite is the case.

\section{Signal detection theory in Hilbert space}

doi:10.1017/S0140525X12002865

\section{Marcus Vinícius C. Baldo}

Department of Physiology and Biophysics, "Roberto Vieira" Laboratory of Sensory Physiology, Institute of Biomedical Sciences, University of São Paulo, 05508-900 São Paulo, SP, Brazil.

baldo@usp.br

Abstract: The Hilbert space formalism is a powerful language to express many cognitive phenomena. Here, relevant concepts from signal detection theory are recast in that language, allowing an empirically testable extension of the quantum probability formalism to psychophysical measures, such as detectability and discriminability.

The Hilbert space formalism seems to be a suitable framework to accommodate the experimental richness of cognitive phenomena. The target article by Pothos \& Busemeyer (P\&B) accomplishes the impressive task of providing, in as simple a way as possible, the theoretical grounds as well as the empirical underpinnings of a probabilistic model capable of grasping many aspects of human cognition. The contribution of this commentary is to point out that important concepts arising from signal detection theory (SDT) can be easily recast into the language of quantum probability. If useful, this addition to P\&B's model might be used to describe several phenomena involved in perceptual detectability and discriminability, enlarging the theoretical reach of their proposal and offering new alternatives to verify its empirical content.

SDT is a powerful tool that has been very successful in many areas of psychological research (Green \& Swets 1966; Macmillan \& Creelman 2005). Originally stemming from applications of statistical decision theory to engineering problems, classical SDT has been reframed over the years under many different assumptions and interpretations (Balakrishnan 1998; DeCarlo 1998; Parasuraman \& Masalonis 2000; Pastore et al. 2003; Treisman 2002). However, irrespective of their formulation, signal detection theories rely on two fundamental performance measures: sensitivity and bias. Whereas sensitivity refers to the ability of an observer to detect a stimulus or discriminate between two comparable stimuli, response bias implies a decision rule or criterion, which can favor the observer's response in one direction or another.

As in standard SDT and its sequels, in this commentary sensitivity and bias are also derived from the probabilities of hits, $p$ $(\mathrm{H})$, and of false alarms, $p(\mathrm{FA})$, defined as the probability of indicating the presence of a stimulus when it is present or absent. Two other quantities, the probabilities of misses, $p(\mathbf{M})$, and correct rejections, $p(\mathrm{CR})$, are complementary, respectively, to $p(\mathrm{H})$ and $p(\mathrm{FA}): p(\mathrm{H})+p(\mathbf{M})=1=p(\mathrm{FA})+p(\mathrm{CR})$.

To translate these concepts into the language of Hilbert space, we need to represent a perceptual state by a state vector $|\Psi\rangle$. This vector denotes the perceptual state of an observer immediately after a trial wherein either the background noise was presented in isolation $\left(\left|\Psi_{n}\right\rangle\right)$ or the noisy background was superimposed with the target stimulus $\left(\left|\Psi_{n+s}\right\rangle\right)$. These state vectors are formed by a linear combination of the $|y e s\rangle$ and $\mid$ no $\rangle$ vectors, an orthogonal vector basis in the present Hilbert space. The components of a state vector along the one-dimensional subspaces that represent the two possible outcomes (either the response "yes" or the response "no" to the question "was the stimulus present?") are given by the projection of the state vector onto the subspaces for "yes" and "no," which are spanned by the basis vectors (Fig. 1). Denoting by $\mathrm{P}_{\text {yes }}$ and $\mathrm{P}_{\text {no }}$ the projection operators (projectors) onto the subspaces spanned by the basis vectors, the components $\mathrm{C}_{1}$ and $\mathrm{C}_{2}$ of a state vector $|\Psi\rangle=\mathrm{C}_{1} \mid$ yes $\rangle+\mathrm{C}_{2} \mid$ no $\rangle$ are given by $\mathrm{C}_{1}=\| \mathrm{P}_{\text {yes }}|\Psi\rangle \|$ and $\mathrm{C}_{2}=\| \mathrm{P}_{\mathrm{no}}|\Psi\rangle \|$.

As we can see in Figure 1, the probabilities of hits, misses, false alarms, and correct rejections are obtained by the action of the projectors $\mathrm{P}_{\text {yes }}$ and $\mathrm{P}_{\text {no }}$ on the state vectors $\left|\Psi_{n}\right\rangle$ and $\left|\Psi_{n+s}\right\rangle$. These probabilities can be computed by means of the following "statistical algorithm" (Hughes 1989):

$$
\begin{aligned}
\mathrm{p}(\mathrm{H}) & =\left\langle\psi_{n+s} \mid \mathrm{P}_{\text {yes }} \psi_{n+s}\right\rangle=\| \mathrm{P}_{\text {yes }}\left|\psi_{n+s}\right\rangle \|^{2} \\
\mathrm{p}(\mathrm{FA}) & =\left\langle\psi_{n} \mid \mathrm{P}_{\text {yes }} \psi_{n}\right\rangle=\| \mathrm{P}_{\text {yes }}\left|\psi_{n}\right\rangle \|^{2} \\
\mathrm{p}(\mathrm{M}) & =\left\langle\psi_{n+s} \mid \mathrm{P}_{\mathrm{no}} \psi_{n+s}\right\rangle=\| \mathrm{P}_{\text {no }}\left|\psi_{n+s}\right\rangle \|^{2} \\
\mathrm{p}(\mathrm{CR}) & =\left\langle\psi_{n} \mid \mathrm{P}_{\mathrm{no}} \psi_{n}\right\rangle=\| \mathrm{P}_{\mathrm{no}}\left|\psi_{n}\right\rangle \|^{2}
\end{aligned}
$$

Analogously to SDT, two measures can be extracted from the vector representation of a perceptual state: an angle, $\boldsymbol{\delta}$, which evaluates the separation between the two state vectors, $\left|\Psi_{n}\right\rangle$ and $\left|\Psi_{n+s}\right\rangle$, gives a measure of sensitivity; another angle, $\boldsymbol{\chi}$, which 
Commentary/Pothos \& Busemeyer: Can quantum probability provide a new direction for cognitive modeling?

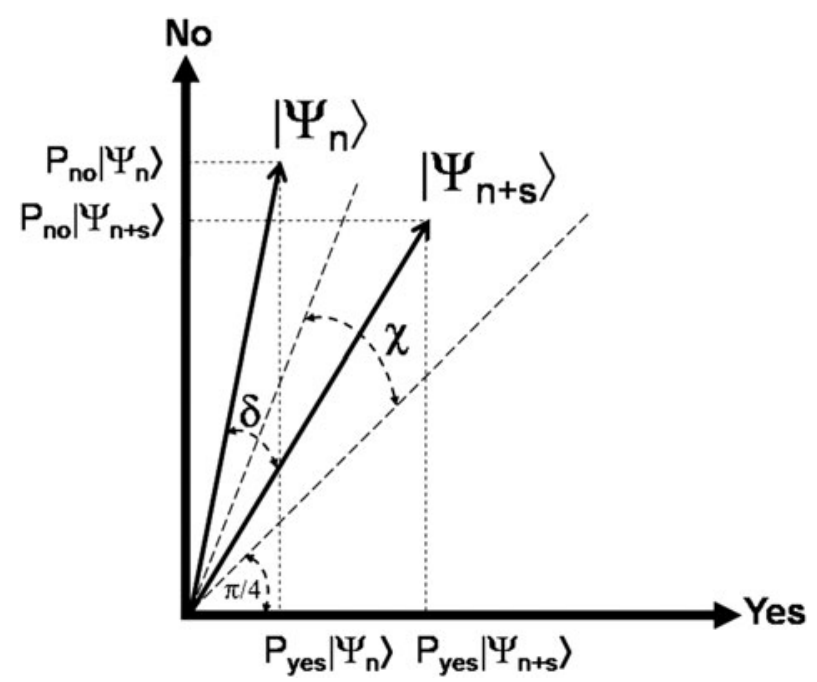

Figure 1 (Baldo). A representation of perceptual states in Hilbert space. The state vectors $\left|\Psi_{n}\right\rangle$ and $\left|\Psi_{n+s}\right\rangle$ represent the perceptual state of an observer exposed, respectively, to noise only or to a signal presented on a noisy background. On each condition, the probability of the observer reporting the presence ("yes") or absence ("no") of the signal is given by squaring the projection of each vector onto the respective basis vector (see equations 1-4).

evaluates the location of the state vectors as a whole in relation to the basis vectors, gives a measure of response bias. In two dimensions, these quantities can be calculated from the pair of probabilities $p(\mathrm{H})$ and $p(\mathrm{FA})$ :

$$
\begin{aligned}
& \boldsymbol{\delta}=\arccos \left[\mathrm{p}(\mathrm{FA})^{1 / 2}\right]-\arccos \left[\mathrm{p}(\mathrm{H})^{1 / 2}\right] \\
& \boldsymbol{\chi}=(1 / 2)\left(\arccos \left[\mathrm{p}(\mathrm{FA})^{1 / 2}\right]+\arccos \left[\mathrm{p}(\mathrm{H})^{1 / 2}\right]\right)-\pi / 4
\end{aligned}
$$

Similarly to SDT, the measures of sensitivity and bias in Hilbert space are also, respectively, the subtraction and addition of terms given by nonlinear transformations of hit and false alarm rates. In Equation 6 , the term $-\pi / 4$ is added only to set $\chi=0$ for an unbiased observer. Analogously to SDT, $\chi>0$ means a stricter criterion (a response bias toward lower hit and false alarm rates), whereas $\chi<0$ means a more lax criterion (a bias toward higher hit and false alarm rates). Figure 2, which resembles a receiver operating characteristic (ROC) representation, shows a family of isosensitivity curves in which the decision criterion $\chi$ changes as a function of the hit and false alarm rates along five different magnitudes of the sensitivity measure, from $\boldsymbol{\delta}=0$ to $\boldsymbol{\delta}=\pi / 3$.

This geometric representation of SDT in Hilbert space can help us visualize many perceptual phenomena. As one example, the modulatory effect of attention could be conceived of as the action of a unitary operator A on the noise-plus-signal state vector, $\left|\Psi_{n+s}\right\rangle$, the noise-only state vector, $\left|\Psi_{n}\right\rangle$, or both, always resulting in a rotation that, without changing their magnitudes, moves them away from each other, thus increasing the sensitivity $\boldsymbol{\delta}$. The physiological interpretation of the attentional operator A would be the enhancement of the signal, the suppression of noise, or both, depending on which vectors the operator's action takes place on. However, a unitary operator that acts on both vectors at the same time, and whose action results in a rotation that preserves their angular separation $\boldsymbol{\delta}$, can be viewed as a change in the decision criterion $\chi$ only.

In conclusion, the idea of this commentary is to accommodate in the language of Hilbert spaces some important concepts that are extremely valuable in the experimental exploration of psychophysical and perceptual phenomena. Whether this attempt bears

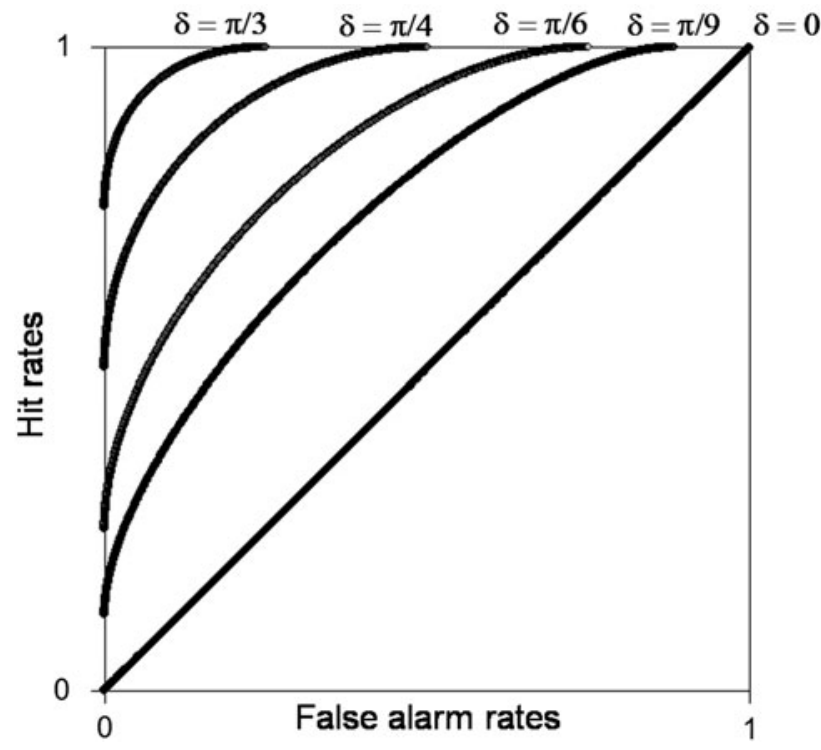

Figure 2 (Baldo). A ROC curve in Hilbert space. Each line is an iso-sensitivity curve that provides the proportion of hit and false alarm rates along a constant sensitivity measure $(\boldsymbol{\delta})$. In the present model, the sensitivity measure is given by the angle, $\boldsymbol{\delta}$, that separates the two state vectors $\left|\Psi_{n}\right\rangle$ and $\left|\Psi_{n+s}\right\rangle$.

some value, or it turns out to be only a mathematical exercise, is an empirical question.

\section{ACKNOWLEDGMENTS}

This work was partially supported by the São Paulo Research Foundation and the National Counsel for Scientific and Technological Development (Brazil). I also thank Nestor Caticha for fruitful discussions on this topic.

\section{Can quantum probability help analyze the behavior of functional brain networks?}

\section{doi:10.1017/S0140525X12002877}

\section{Arpan Banerjee and Barry Horwitz}

Brain Imaging and Modeling Section, Voice, Speech and Language Branch, National Institute on Deafness and other Communication Disorders, National Institutes of Health, Bethesda, MD 20892-1402.

Arpan.Banerjee@nih.gov

horwitzb@mail.nih.gov

http://www.nidcd.nih.gov/research/scientists/pages/horwitzb.aspx

Abstract: Pothos \& Busemeyer (P\&B) argue how key concepts of quantum probability, for example, order/context, interference, superposition, and entanglement, can be used in cognitive modeling. Here, we suggest that these concepts can be extended to analyze neurophysiological measurements of cognitive tasks in humans, especially in functional neuroimaging investigations of large-scale brain networks.

We agree in general with the views expressed in the target article by Pothos \& Busemeyer $(\mathrm{P} \& \mathrm{~B})$ about the application of quantum probability $(\mathrm{QP})$ to cognitive science. Moreover, we believe that the mathematical framework of QP can be used as well to interpret brain network interactions underlying cognitive tasks, and that this should be further explored in the future. Here, we emphasize that the concept of functional brain network embodies notions similar to those of QP, such as entanglement, interference, and incompatibility.

Modern neuroimaging experimental designs are often based on the idea of studying a complex cognitive task as a sum of multiple sensory/cognitive factors that have corresponding processing 
modules in the brain (Sternberg 2011). For example, to study auditory-visual integration, one would design unimodal control tasks (factors) employing visual and auditory stimuli separately, and examine the change of brain responses during presentation of combined visual-auditory stimuli (Molholm et al. 2004). Another example comes from investigating the neural correlates of bimanual coordination: execution of movements with both limbs, for example, two hands or two feet. Here, unimanual movements (movement with one limb at a time) serve as factors for studying the neural correlates of bimanual movements (Banerjee et al. 2012a; Debaere et al. 2001).

A fundamental problem with factor-based approaches is that they implicitly assume that each cognitive component triggers an additional neural correlate that is the same, irrespective of the cognitive or physiological context (Banerjee et al. 2008). For example, while studying multisensory integration, one often assumes that the same brain response during an auditory stimulus presentation is expected to be triggered in auditory areas when a visual stimulus is tagged with the auditory stimulus presentation as a combined auditory-visual stimulus (Molholm et al. 2004). The reader of $\mathrm{P} \& \mathrm{~B}$ will note that this assumption is identical to the concept of "compatibility," and is not appropriate for studying the neural basis of human sensory and cognitive processes that are adaptive to even subtle changes of context (McIntosh 2004).

Furthermore, an implicit assumption in factor-based neuoimaging designs is that any residual (e.g., bimanual: sum of left and right unimanual) brain activity comes from recruitment of additional brain areas related only to the missing factor not addressed by the control tasks (Banerjee et al. 2008). Hence, the possibility of "interference" of control-related networks during the active cognitive task is ignored by factor-based designs. This approach thus disregards the "degenerate" nature of cognitive neural systems; different networks can coordinate to perform the same tasks (Price \& Friston 2002; Tononi et al. 1999). Degeneracy is an omnipresent phenomenon of complex biological systems, and the search for measures to characterize degenerate neural systems is an ongoing area of research (Edelman \& Gally 2001; Tononi et al. 1999). Current neuroscience research indicates that there are two distinct conceptualizations of neural degeneracy: structural and functional. The same neural structures can give rise to different functions (structural) and different neural structures can generate the same function (functional) (McIntosh 2004; Price \& Friston 2002). An example of the former was observed in an electroencephalographic (EEG) study of bimanual coordination (Banerjee et al. 2012a) in which participants were asked to move their left or right fingers independently and simultaneously in different trials at specific frequencies by syncing with a rhythmic visual stimulus. During execution of stable bimanual coordination patterns, neural dynamics were dominated by temporal modulation of unimanual networks. An example of functional degeneracy occurred in an investigation of the temporal microstructure of a long-term memory retrieval process (Banerjee et al. 2012b). Here, magnetoencephalographic (MEG) data were used to examine the network recruitment of auditory areas during a visual-auditory paired associate task compared with a visualvisual paired associate task. It was found that visual-visual and visual-auditory memory recollection involved equivalent network components without any additional recruitment during an initial period of the sensory processing stage, which was then followed by recruitment of additional network components for modality-specific memory recollection.

$\mathrm{P} \& \mathrm{~B}$ point out that the QP concept of "entanglement" plays a crucial role in understanding cognitive behavior. The mathematical framework of expressing "entanglement" involves quantifying the evolution of correlated state variables, and hence is pertinent for the study of functional brain networks. Data acquired by functional neuroimaging (i.e., functional magnetic resonance imaging [fMRI] and EEG/MEG) are being investigated by cognitive neuroscientists using a variety of network-based mathematical tools, such as functional connectivity (Beckmann et al. 2005; Horwitz et al. 1992), effective connectivity (Friston et al. 2003; McIntosh et al. 1994), and graph theory (Bullmore \& Sporns 2009). A critical point with respect to functional networks is that as all nodes are functionally linked to one another, any alteration in even a single link results in alterations throughout the network (Kim \& Horwitz 2009). In essence, one can say that brain network nodes (regions) that may not be anatomically connected and are physically separated by large distances can become "entangled" with one another to facilitate task execution. This aspect of network behavior has important ramifications for the way in which functional brain imaging data are interpreted in comparing tasks, or patients and healthy subjects (Kim \& Horwitz 2009).

To conclude, we think that the insights that QP brings to cognitive science, as reviewed by $\mathrm{P} \& \mathrm{~B}$, are likely to be as significant when used for interpretation of brain network analysis. An examination of the recent brain imaging literature suggests that such an approach already is under way (Banerjee et al. 2012a; 2012b; Noppeney et al. 2004). Although the words used by functional brain imagers to describe their methods differ from the language of QP, we have attempted to point out that there are strong similarities in the fundamental mathematical problem each strives to solve.

\section{ACKNOWLEDGMENT}

This work was supported by the Intramural Research Program of the National Institute on Deafness and Other Communications Disorders.

\section{Uncertainty about the value of quantum probability for cognitive modeling}

\author{
doi:10.1017/S0140525X12002889
}

\section{Christina Behme \\ Department of Philosophy, Dalhousie University, Halifax, NS B3H 4P9 Canada. \\ Christina.Behme@dal.ca}

Abstract: I argue that the overly simplistic scenarios discussed by Pothos \& Busemeyer $(\mathrm{P} \& \mathrm{~B})$ establish at best that quantum probability theory (QPT) is a logical possibility allowing distinct predictions from classical probability theory (CPT). The article fails, however, to provide convincing evidence for the proposal that QPT offers unique insights regarding cognition and the nature of human rationality.

Pothos \& Busemeyer $(\mathrm{P} \& \mathrm{~B})$ propose that many existing models fail to account for all aspects of cognitive processing, and discuss a number of phenomena for which the formal framework of classical probability theory (CPT) seems to fail. However, virtually all examples were based on hypothetical reasoning or experiments under artificial laboratory or Gallup poll conditions. Therefore, even if quantum probability theory (QPT) can account better for some of these phenomena, it is not clear that the much stronger claim that QPT offers unique insights regarding cognition and the nature of human rationality is supported at this stage. To support this stronger claim, $\mathrm{P} \& \mathrm{~B}$ would have needed to establish that the cases discussed are paradigm cases of human cognition and not artifacts of the experimental setup. Further, to argue for QPT, P\&B need to do more than observe that QPT predictions differ from CPT ones. Before arguing "that the quantum approach to cognition embodies all the characteristics of good theory" (sect. 6.4) $\mathrm{P} \& \mathrm{~B}$ need to show in detail over a wide range of cases that specific numerical QPT predictions are correct.

One rather serious problem is the informal and vague presentation throughout. There seem to be two components/aspects to $\mathrm{P} \& \mathrm{~B}$ 's QP model, distinguishing it from CP. One aspect is the issue of and motivation for vectors space models, which could be real but, nevertheless, would have associated non-classical logical operators (disjunction, conjunction, negation, implication) 
that could account for non-commutativity effects. The second aspect is the issue of and motivation for complex vector spaces. $\mathrm{P} \& \mathrm{~B}$ attempt to justify their preference for QPT by stressing the value of projection operators. But projection operators can be defined on real (in fact, on any) vector space. Real vector spaces are used in many areas such as machine learning and information retrieval (e.g., van Rijsbergen, 2004; Widdows 2004). The key concepts required for similarity matching include inner product, length, angle, and projection. But none of those are unique to complex vector spaces. However, $\mathrm{P} \& \mathrm{~B}$ discuss these notions in the context of finite-dimensional complex vector spaces; their Hilbert spaces. As the arguments seem to support only real vector space models over classical set-theoretic models, $\mathrm{P} \& \mathrm{~B}$ would need an independent argument showing that similarity matching for cognition requires complex numbers. $\mathrm{P} \& \mathrm{~B}$ have not shown that any of the examples require quantum probability amplitudes, which involve complex numbers. For example, the concept of superposition does not require complex values but applies to linear systems quite generally and hence to vector spaces. Conflating notions that need to be kept separate makes it difficult to evaluate whether the application of quantum probability is justified.

Turning to the work discussed, it appears that especially the Linda case, on which much argumentative burden rests, is problematic for several reasons. First, the case is not based on an actual person but on one that had been "constructed to be representative of an active feminist and unrepresentative of a bank teller" (Tversky \& Kahneman 2004, p. 227). Reasoning under such artificial conditions seems hardly a prototypical case of rational decision making. Further, not all participants committed the conjunction fallacy and the authors of the study point out that "because individuals who have different knowledge or hold different beliefs must be allowed to assign different probabilities to the same event no single value can be correct for all people" (Tversky \& Kahneman 2004, p. 221). It is likely that some participants in such experiments rely, at least to some degree, on incorrect background information. This might explain the violation of the law of total probability, the conjunction fallacy. Undoubtedly, these experiments establish that models that assume fully rational agents do not apply to humans. But they do little to indicate whether QPT is a better model for human cognition than is CPT. Possibly non-QPT models can account for the observed anomalies if we assume that the reasoner relied to some degree on incorrect information and/or did not reason fully rationally. Similarly, the artificial conditions of the one-shot prisoner dilemma seem to interfere in some (but not all) individuals with rational decision making (Shafir \& Tversky 2004). But more realistic reiterated prisoner dilemma tasks lead to different results (e.g., Axelrod \& Hamilton 1981; Nowak \& Sigmund 1992). These cases seem to establish that rationality breaks down in some cases but not in others. This is very different from quantum physics, in which it is always the case that "knowledge of the position of a particle imposes uncertainty on its momentum" (sect. 1.1). Hence, the analogy between cognition and quantum physics might be much weaker than $\mathrm{P} \& \mathrm{~B}$ suggest. They assume that any ad hoc models are inferior to a single, uniform mathematical framework (whether it be CPT or QPT). Given the complexity of human decision making, however, ad hoc models that make better predictions might be preferable over principled frameworks that make inferior predictions.

Another area of concern is the claim that "there are situations in which the distinctive features of QP provide a more accurate and elegant explanation for empirical data" (sect. 1.2). However, the specific examples discussed involve only the interaction between two parameters. In such a constrained setting, it may be true that for quantum probability theory "the relevant mathematics is simple and mostly based on geometry and linear algebra" (sect. 1.2). But it is not clear at all whether and how the proposed models can account for the multiply intertwined interactions between existing knowledge and any imaginable problem solving task. Take the discussion of the interference effects between "happiness" and "employment." The superficial and sketchy discussion of the superposition effects of just these two variables seems to support the conclusion that emotional judgments are the results of constructive processes. However, anyone accepting the claim that QPT "fundamentally requires a constructive role for the process of disambiguating a superposition state" (sect. 2.1), has to ask whether such disambiguation is possible in realistic cases in which all factors that affect the emotional state of a person need to be considered. P\&B seem to have no principled way of determining whether two attributes are incompatible. But such a distinction would be required before we can decide whether QPT is needed to replace CPT. The authors observe that classical optimality "assumes a measurable, objective reality and an omniscient observer" (sect. 5). This assumption is problematic. But this has been discussed in the philosophical literature at least since Hume (1751/1999; see also Cartwright 1999), and current models do not attempt to make predictions for all aspects of the "real, noisy, confusing, ever-changing, chaotic world" (sect. 5). Many questions regarding the best model for cognition remain open and it seems premature to assert, "the QP cognitive program...is clearly an important direction for future research" (sect. 6.3, emphasis added).

ACKNOWLEDGMENTS

I thank David Johnson and Robert Levine for their detailed comments. All remaining errors are mine.

\section{The (virtual) conceptual necessity of quantum probabilities in cognitive psychology}

\author{
doi:10.1017/S0140525X12002890
}

\section{Reinhard Blutner ${ }^{\mathrm{a}}$ and Peter beim Graben ${ }^{\mathrm{b}}$}

alLLC, Universiteit van Amsterdam, Amsterdam, 1090 GE, The Netherlands; ${ }^{\mathrm{b}}$ Department German Language and Linguistics, Humboldt Universität zu Berlin, 10099 Berlin, Germany.

k.r.blutner@uva.nl

prbeimgraben@googlemail.com

http://www.blutner.de/ http://www.beimgraben.info/

Abstract: We propose a way in which Pothos \& Busemeyer $(\mathrm{P} \& \mathrm{~B})$ could strengthen their position. Taking a dynamic stance, we consider cognitive tests as functions that transfer a given input state into the state after testing. Under very general conditions, it can be shown that testable properties in cognition form an orthomodular lattice. Gleason's theorem then yields the conceptual necessity of quantum probabilities (QP).

Pothos \& Busemeyer (P\&B) discuss quantum probabilities (QP) as providing an alternative to classical probability $(\mathrm{CP})$ for understanding cognition. In considerable detail, they point out several phenomena that CP cannot explain, and they demonstrate how QP can account for these phenomena. An obvious way to downplay this chain of arguments is by demonstrating that in addition to $\mathrm{CP}$ and QP models, alternative approaches are possible that could also describe the phenomena without using the strange and demanding instrument of QP. For example, one could argue that the conjunction puzzle can be resolved by simple heuristics (Gigerenzer 1997), and the question ordering effects can be resolved by query theory (Johnson et al. 2007).

A general strategy to invalidate such criticism is to look for a universal motivation of QP that is based on fundamental (architectural) properties of the area under investigation. As Kuhn (1996) clarified, such basic assumptions constituting a theoretical paradigm normally cannot be justified empirically. Basic assumptions that concern the general architecture of the theoretical system (paradigm) are called design features. Using a term that is common in the generative linguistic literature (Chomsky 1995; 2005), we will describe properties that are consequences of such design features as applying with (virtual) conceptual necessity. 
Commentary/Pothos \& Busemeyer: Can quantum probability provide a new direction for cognitive modeling?

We believe that $\mathrm{P} \& \mathrm{~B}$ could further strengthen their argument by demonstrating that quantum probabilities are such a (virtual) conceptual necessity. This can be achieved by adopting the recent developments of "dynamification" in logic (van Benthem 2011) and cognition (Barsalou 2008) in which cognitive actions play a much more significant role than do "static" propositions. Among others (including Atmanspacher et al. 2002), Baltag and Smets (2005) have given a complete axiomatization for quantum action, based on the idea of a quantum transition systems. In this view, the states of a system are identified with the actions that can be performed on the states. In physics, the relevant actions are measurements. In cognitive psychology, actions correspond to tests that subjects conduct (yes/no questions in the simplest case). Basically, a quantum dynamic frame is characterized by a set of states $\Sigma$ and a system T of subsets of $\Sigma$ called testable properties. Each testable property $A \in T$ is characterized by a unique (partial) transfer function $P_{A}$, which describes how testing of $A$ changes an input state $s \in \Sigma$. The system of testable properties and the corresponding transfer functions are characterized by some plausibility conditions. For example, the testable properties are closed under arbitrary intersection, states are testable, testing a true property does not change the state $[s \in A$ implies $\left.P_{A}(s)=s\right]$, and repeatability $\left(\mathrm{P}_{A}^{2}=\mathrm{P}_{A}\right)$. Further, there are more technical axioms such as self-adjointness and a covering law.

Restricting testable properties in this way, Baltag and Smets (2005) were able to prove (based on earlier work by Piron [1976]) that quantum dynamic frames are isomorphic to the lattice of the closed subspaces of a Hilbert space (with transfer functions as projection operators). In the Baltag/Smets approach, two states $s$ and $t$ are considered orthogonal if no measurement can transfer $s$ into $t$. Properties $A$ and $B$ are orthogonal if all states of $A$ are orthogonal to all states of $B$. If $A$ and $B$ are orthogonal, the corresponding subspaces of the Hilbert space are orthogonal as well (and vice versa). Mathematically, probabilities are totally additive measure functions, in the classical case based on Boolean algebras and in the quantum case based on orthomodular lattices. The underlying algebra is decisive for the properties of the resulting measure function. In the quantum case, Gleason's theorem states that the corresponding measure functions can be expressed by the squared length of the projections of a given state $s$ (or more generally, as the convex closure of such functions; for details, see the original article [Gleason 1957], and for a constructive proof see Richman \& Bridges [1999]), that is, as QP.

Our view is further supported by P\&B's speculations about implications for brain neurophysiology. In the algebraic approach, even classical dynamic systems, such as neural networks, could exhibit quantum-like properties in the case of coarse-graining measurements, when testing a property cannot distinguish between epistemically equivalent states. Beim Graben and Atmanspacher (2009) used this "epistemic quantization" for proving the possibility of incompatible properties in classical dynamic systems. In neuroscience, most measurements, such as electroencephalography or magnetic resonance imaging, are coarse-grainings in this sense. Therefore, the Baltag/Smets approach has direct implications for brain neurophysiology, without needing to refer to a "quantum brain" as indicated by P\&B.

Summarizing, the Baltag/Smets approach provides an independent motivation of QP, which is not based on particular phenomena but rather on independently motivated general conditions concerning the dynamics of testing. All the conditions needed for the proof are formulated in purely dynamic terms. This makes quantum dynamic frames especially appealing for psychological approaches formulating operational cognitive laws. Recent work by Busemeyer and Bruza (2012), Trueblood and Busemeyer (2011), and Blutner (2012) is in this spirit. Based on a dynamic picture of propositions and questions and hence on the design principles of cognitive architecture, we state that QP is a virtual conceptual necessity. Needless to say, we regain CP (Kolmogorovian) by assuming that no test is changing the state being tested.

\section{On the quantum principles of cognitive learning}

\section{doi:10.1017/S0140525X12002919}

\author{
Alexandre de Castro \\ Laboratório de Matemática Computacional-CNPTIA/Embrapa - Campinas, \\ 13083-886 SP, Brazil. \\ alexandre.castro@embrapa.br \\ http://www.cnptia.embrapa.br
}

Abstract: Pothos \& Busemeyer's (P\&B's) query about whether quantum probability can provide a foundation for the cognitive modeling embodies so many underlying implications that the subject is far from exhausted. In this brief commentary, however, I suggest that the conceptual thresholds of the meaningful learning give rise to a typical Boltzmann's weighting measure, which indicates a statistical verisimilitude of quantum behavior in the human cognitive ensemble.

The principles of superposition and entanglement are central to quantum physics. Quantum superposition is commonly considered to be a mapping of two bit states into one. Mathematically, we can say that it is nothing more than a linear combination of classical (pure) states. As to the quantum entanglement, it refers to a short- or long-range operation in which a strongly correlated state, a mixed state, is built from pure states. An important feature of this mixed state is that it cannot be represented by a tensor product of states, and once such an entangled system is constructed, it cannot be dissociated (Dirac 1999).

In their target article, Pothos \& Busemeyer (P\&B) elegantly argue that there may be quantum principles - notably superposition and entanglement - at play in the context of human cognitive behavior. They also draw attention to the pertinent idea that the concept of quantum likelihood can provide a novel guidance in cognitive modeling. Viewed in these terms, I tend to agree with $\mathrm{P} \& \mathrm{~B}$ because I, too, have identified both superposition and entanglement from the cognitive premises formulated within the process of subsumption (assimilation) of information proposed by Ausubel (1963; 1968).

From the point of view of the process of subsuming information, the material meaningfully incorporated within an individual's cognitive structure is never lost, but a process called "forgetting" takes place in a much more spontaneous manner, because it is a continuation of the very process of associative subsumption by which one learns. This forgetting state is an obliterative stage of the subsumption characterized by Ausubel as "memorial reduction to the least common denominator" (Brown 2000). This "memorial reduction" required for the acquisition of new meanings (knowledge) is clearly (and remarkably) a conceptual process of quantum superposition of mental states, and, consequently, this cognitive behavior can be generically expressed by a quantum operation of retention of information, a cognitive squeeze, as follows: $\mid$ bit $\rangle+|b i t\rangle=\mid$ $q u b i t\rangle$. In addition, Ausubel (1963) claimed that, when the obliterative stage of subsumption begins, "specific items become progressively less dissociable as entities in their own right until they are no longer available and are said to be forgotten."

This "forgetting" theorized by Ausubel seems to reflect very well the entanglement included in the central idea of quantum cognition raised by P\&B. In passing, Vitiello’s work (1995) quoted in the target article also addressed the squeeze of information and the forgetting dynamics to describe the cognitive behavior, although that work does not properly refer to Ausubelian subsumption of information. Nevertheless, more in line with Ausubel's premises, Brookes' pioneering work (1980) on the cognitive aspects in the information sciences (Bawden 2008) provides a quantitative sharp bias of meaningful learning, albeit one seldom examined from this perspective (Neill 1982; Cole 1997; 2011). Most of the works found in the literature quote Brookes' fundamental equation of information science, $K(S)$ $+I \rightleftarrows K(S+\Delta \mathrm{S})$ - here assumed as an obliterative synthesis that exhibits short-term instability - as merely a shorthand description of knowledge transformation, wherein the state of mind $K(S)$ 
changes to another state $K(S+\Delta S)$, because of an input of information $I$, being $\Delta S$ as an indicator of the effect this transformation (Bawden 2011; Cornelius 2002).

On the other hand, Brookes (1981) - although in an incipient approach - conjectured outright that the recipient knowledge structure included in the fundamental equation could be quantitatively treated, which in a subjacent manner, links his work to the assimilative schema of information expressed by the Ausubelian symbolic quantities, even though these quantities are situated in a semiquantitative pictorial landscape (Novak 2011; Moreira 2011; Seel 2012). In support of this idea, Todd (1999) also advocated that the unit of information embedded in Brookes' theory is a concept derived from Ausubel's learning theory. For such reasons, I am convinced that Brookes' equation faithfully shapes the Ausubelian retention of information, or, more specifically, the superposition and entanglement of information underlying the subsumption.

Interestingly, if we take into account that information is the boundary condition of the human cognitive system - and if we continue to perceive knowledge from a Nietzschean viewpoint, in which subject and object are confused-then the reciprocal reckoning of Brookes' equation, $K(S+\Delta S) \rightarrow K(S)+I$, in addition to providing a typical scenario of information retention, also seems to give us a symbolic (and quantum) translation of Jose Ortega y Gasset's famous maxim, "I am I plus my circumstances," which Gasset (1998) placed at the metaphysical core of his epistemological approach of perspectivism.

Brookes himself addressed a peculiar notion of perspectivism in his work. In a pioneering way, Brookes (1981) suggested in a reductionist geometric context - although without clarification - a rough sketch, a skeleton, of a logarithmic equation to represent the carrying of information into the human mind on the same basis as Hartley's law (Seising 2010), seeing that Hartley's law-predecessor to Shannon's idea of channel capacity - had, up to that time, been designed solely to handle information in a purely physical system. However, albeit Brookes has made a valuable contribution by suggesting a Hartley-like behavior for information processing in the mind, he was not able to identify the appropriate cognitive variables for the implementation of his physicalistic approach from the perspectivism.

I showed in a recent preprint (Castro 2013) that the conceptual schema of meaningful learning leads directly to a ShannonHartley-like model (Gokhale 2004), and that this model can be interpreted from basilar cognitive variables, such as information and working memory. Moreover, starting this learning schema, I have found that the ratio between the two mental states given by the Brookes' fundamental equation of information science is as follows: $K(S) / K(S+\Delta S) \geq e^{-\Delta E / k_{B} T}$, where $\Delta E$ is the free energy of the ensemble, $k_{B}$ is Boltzmann's constant, and $T$ is the absolute temperature. The so-called Boltzmann factor, $e^{-\Delta E / k_{B} T}$, is a weighting measure that evaluates the relative probability of a determined state occurring in a multi-state system (Carter 2001) - that is, it is a "non-normalized probability" that needs to be "much greater" than unity for the ensemble to be described for non-quantum statistics; otherwise, the system exhibits quantum behavior.

As a result, this calculation shows that the internalization of one unit of information into an individual's mental structure gives rise to a Boltzmann "cognitive" factor $\leq 2$, which provides us a circumstantial evidence that the subsumption of new material, as a cognitive process, requires a quantum-statistical treatment, such as $\mathrm{P} \& \mathrm{~B}$ have proposed.

\section{Cold and hot cognition: Quantum probability theory and realistic psychological modeling}

\section{doi:10.1017/S0140525X12002907}

\section{Philip J. Corr}

School of Psychology, University of East Anglia, Norwich Research Park, Norwich NR4 7TJ, United Kingdom.

p.corr@uea.ac.uk

http://www.ueapsychology.net/
Abstract: Typically, human decision making is emotionally "hot" and does not conform to "cold" classical probability (CP) theory. As quantum probability (QP) theory emphasises order, context, superimposition states, and nonlinear dynamic effects, one of its major strengths may be its power to unify formal modeling and realistic psychological theory (e.g., information uncertainty, anxiety, and indecision, as seen in the Prisoner's Dilemma).

Classical probability (CP) theory has struggled to provide a comprehensive, sometimes even adequate, description and explanation of human decision making. This conclusion has pervaded psychology and related disciplines, for example in economics, where the notion of a rational single-type homo economicus is fast falling out of favour and being replaced by one that highlights the heterogeneity of economic agents and their decision- making processes. Many behavioural scientists doubt that formal modeling based on CP principles can describe and explain even relatively simple laboratorybased behaviour, let alone the everyday examples that, typically, entail some degree of emotional activation by virtue of the different payoffs associated with different possible outcomes. The fact that different outcomes exist is, itself, sufficient to induce conflictrelated emotion, thus rending typical human decision making "hot."

Whatever the ultimate value of quantum probability (QP) theory, it represents a promising way forward to model the computations of cognition involved in complex psychological situations. The need for such a formal modeling theory is highlighted by the trend in recent years towards an integration of "cold" cognition with "hot" (emotional) processes and decision making, as seen in the "cognition-emotion" literature. CP theory is poorly equipped to meet this challenge. This commentary explores the potential of QP theory to provide a general modeling approach for realistic psychology theory.

In the typical laboratory situation, the experimenter tries to control all extraneous variables to isolate only those of interest; however, in the case of human studies, internal states of the participant cannot be controlled. Participants bring to the experimental situation their own expectations, desires, and habitual modes of thinking and behaving; and, worse still for the experimentalist, these factors differ among individuals. This fact is seen readily in experimental games studied by behavioural economists, in which understanding of behaviour is impoverished by failing to account for dispositional differences in preferences and personality (Ferguson et al. 2011).

The above-mentioned points can easily be illustrated in relation to the "sure thing" principle seen with the prisoner's dilemma problem. The literature shows: (1) knowing that one's partner has defected leads to a higher probability of defection; (2) knowing that one's partner has cooperated also leads to a higher probability of defection; and, most troubling for CP theory, (3) not knowing one's partner's decision leads to a higher probability of cooperation. Everyday equivalents of this situation do not, typically, resemble the decision-making dynamics assumed by CP theory: people have psychological processes that it fails to model, or to even consider to be relevant. For example, in this situation, outcome (1) could simply be (self-serving) retaliation; and in the case of (2) taking the easier route, but, we should wonder, what would happen if the partner was a loved one (e.g., your child)? Scenario (3) is a situation of uncertainty and psychological conflict (at least as perceived by many participants), and we know from the literature that such conflicts lead to cautious, risk-assessing behaviour which should be expected to lower rates of defection (which represents a final decision devoid of any cognitive dithering). Not knowing one's partner's decision is, in psychological if not logical terms, very different to outcomes (1) and (2); therefore, we should expect a different decision outcome.

The main point of the previous discussion is that in a situation of information uncertainty, people's decisions will be influenced by a host of factors, including evaluation of likely payoffs as well as consideration of reputational damage and likely carry-over effects to other situations. Most decision situations outside the 
laboratory do not resemble the constraints of the one-shot experiment, and in this situation people find it difficult to break free from their habitual forms of cognition. In actual fact, most laboratory-based decision-making situations are not as tightly constrained as assumed by the experimenter; for example, the experimenter typically knows the participant's decision and the participants know that the experimenter knows. This is relevant information from the participant's point of view. In designing experimental situations, it is important to make things as simple as possible, but not to the extent that the situation loses touch with psychological reality and, therefore, external validity.

As an example of the consequences of information uncertainty, which is inherent in the prisoner's dilemma problem, psychological entropy has been used to account for its effects. As noted by Hirsh et al. (2012), “As a system's disorder and uncertainty increases, its ability to perform useful work is hampered by reduced accuracy in specifying the current state, the desired state, and the appropriate response for transforming the former into the later" (p. 305). They further note that uncertainty leads to goal-conflict and anxiety, which adds further emotional heat to the cognitive system. And, as with many other processes, in the specific example of conflict-related anxiety, we observe a change in the balance between controlled-reflective and automatic-reflexive behaviour (Corr 2011) which can, and often does, lead to nonlinear dynamic effects of the type predicted by QP theory. In addition, the perception of potential rewards and punishments in the situation trigger prepotent automatic reactions (e.g., in the case of goalconflict anxiety, behavioural inhibition and cognitive rumination; McNaughton \& Corr 2004) which we may well expect to impact on decision-making processes in complex, but unpredictable, ways. Therefore, CP modeling of the prisoner's dilemma problem does not tell us much about how people actually behave in "hot" decision- making situations.

In contrast, one major opportunity of QP theory is the provision of a general computational framework for the modeling of dynamic, and realistic, psychological processes. As Pothos \& Busemeyer (P\&B) observe, QP theory has strong potential in this regard because it takes account of processes that are strongly order and context dependent, recognises that individual states are often superimposition states, and assumes that composite systems are often entangled and cannot be composed into their separate subsystems. It would be valuable for $\mathrm{P} \& \mathrm{~B}$ to consider how their proposals for QP theory might be extended to start to address emotionally hot cognition and behaviour.

\section{Beyond quantum probability: Another formalism shared by quantum physics and psychology}

\author{
doi:10.1017/S0140525X12002920
}

\section{Ehtibar N. Dzhafarov ${ }^{\mathrm{a}}$ and Janne V. Kujala ${ }^{\mathrm{b}}$ \\ ${ }^{a}$ Department of Psychological Sciences, Purdue University, West Lafayette, IN 47907; ' Department of Mathematical Information Technology, University of Jyväskylä, Jyväskylä Fl-40014, Finland. \\ ehtibar@purdue.eduｊvk@iki.fi \\ http://www2.psych.purdue.edu/ ehtibar http://users.jyu.fi/ jvkujala}

\footnotetext{
Abstract: There is another meeting place for quantum physics and psychology, both within and outside of cognitive modeling. In physics it is known as the issue of classical (probabilistic) determinism, and in psychology it is known as the issue of selective influences. The formalisms independently developed in the two areas for dealing with these issues turn out to be identical, opening ways for mutually beneficial interactions.
}

Pothos \& Busemeyer (P\&B) present a compelling case for quantum formalisms in cognitive modeling. This commentary is more of an addendum, about another area in which psychology meets quantum physics, this time as a result of the coincidence of formalisms independently developed and motivated. In quantum physics, they grew from the investigation of the (im)possibility of classical explanation for quantum entanglement, in psychology from the methodology of selective influences. Surprisingly, the meeting occurs entirely on classical probabilistic grounds, involving (at least so far) no quantum probability.

The issue of selective influences was introduced to psychology in Sternberg's (1969) article: the hypothesis that, for example, stimulus encoding and response selection are accomplished by different stages, with durations $A$ and $B$, can be tested only in conjunction with the hypothesis that a particular factor (experimental manipulation) $\alpha$ influences $A$ and not $B$, and that some other factor $\beta$ influences $B$ and not $A$. Townsend (1984) was first to propose a formalization for the notion of selectively influenced process durations that are generally stochastically dependent. Townsend and Schweickert (1989) coined the term "marginal selectivity" to designate the most conspicuous necessary condition for selectiveness under stochastic dependence: if $\alpha \rightarrow A$ and $\beta \rightarrow B$, then the marginal distribution of $A$ does not depend on $\beta$, nor does the marginal distribution of $B$ depend on $\alpha$. This condition was generalized to arbitrary sets of inputs (factors) and outputs (response variables) in Dzhafarov (2003). Selectiveness of influences, however, is a stronger property, as the following example demonstrates. Let $\alpha, \beta$ be binary $0 / 1$ inputs. Consider outputs

$$
A=\alpha+N, B=\beta+(1-2 \alpha \beta) N
$$

where $N$ is standard-normally distributed. The influence of $\alpha$ on $B$ is obvious, but $B$ is distributed as $\operatorname{Norm}($ mean $=\beta$, variance $=1)$, that is, marginal selectivity is satisfied.

It was first suggested in Dzhafarov (2003) that the selectiveness of influences $(\alpha \rightarrow A, \beta \rightarrow B, \ldots)$ means that $A, B, \ldots$ can be presented as, respectively, $f(\alpha, R), g(\beta, R), \ldots$ where $R$ is some random variable and $f, g, \ldots$ are some functions. By now, we know several classes of necessary conditions for selectiveness, that is, ways of looking at joint distributions of $A, B, \ldots$ at different values of inputs $\alpha, \beta, \ldots$ and deciding that it is impossible to represent $A, B, \ldots$ as $f(\alpha, R), g(\beta, R), \ldots$ (Dzhafarov \& Kujala 2010; 2012 b; in press b; Kujala \& Dzhafarov 2008). For special classes of inputs and outputs we also know conditions that are both necessary and sufficient for such a representability. Thus, if $A$, $B, \ldots, X$ and $\alpha, \beta, \ldots, \chi$ all have finite numbers of values, then $\alpha \rightarrow A, \beta \rightarrow B, . ., \chi \rightarrow X$ if and only if the following linear feasibility test is satisfied: the linear system $M Q=P$ has a solution $Q$ with non-negative components, where $P$ is a vector of probabilities for all combinations of outputs under all combinations of inputs, and $M$ is a Boolean matrix entirely determined by these combinations (Dzhafarov \& Kujala 2012b).

In physics, the story also begins in the 1960s, when Bell (1964) found a way to analyze on an abstract level the Bohmian version of the Einstein-Podolsky-Rosen (EPR/B) paradigm. In the simplest case, the paradigm involves two spin-half entangled particles running away from each other. At some moment of time (with respect to an inertial frame of reference) each particle's spin is being measured by a detector with a given "setting," which means a spatial orientation axis. For every axis chosen (input) the spin measurement on a particle yields either "up" or "down" (random output). Denoting these binary outputs A for one particle and $B$ for another, let the corresponding settings be $\alpha$ and $\beta$. Special relativity prohibits any dependence of $A$ on $\beta$ or of $B$ on $\alpha$. Bell formalized classical determinism with this prohibition as the representability of $A, B$ as $f(\alpha, R), g(\beta, R)$, with the same meaning of $f$, $g$, and $R$ as above. Then he derived a necessary 
condition for such a representability, in the form of an inequality involving three settings $(x, y$ for $\alpha$ and $y, z$ for $\beta$ ). The characterizations of Bell's derivation as "one of the profound discoveries of the [20th] century" (Aspect 1999) and even "the most profound discovery in science" (Stapp 1975) are often quoted in scientific and popular literature. A generalization of this inequality to any binary $\alpha$ and $\beta$, known as CHSH inequalities (after Clauser et al. 1969), was shown by Fine (1982) to be a necessary and sufficient condition for representing $A, B$ as $f(\alpha, R), g(\beta, R)$ (assuming marginal selectivity). $\mathrm{CHSH}$ inequalities are a special case of the linear feasibility test mentioned previously as developed in psychology. In physics, this test is described in Werner and Wolf (2001a; 2001b) and Basoalto and Percival (2003).

How does one explain these parallels between the two very different issues? The answer proposed in Dzhafarov and Kujala (2012a; 2012b) is that measurements of noncommuting observables on one and the same particle are mutually exclusive, because of which they can be viewed as different values of one and the same input. Different inputs in the EPR/B paradigm are spin measurements on different particles, whereas input values are different settings for each particle. This is completely analogous to, for example, $\alpha=$ left flash and $\beta=$ right flash in a double-detection experiment being inputs for two judgments ( $\mathrm{A}=\mathrm{I}$ see/don't see $\alpha, \mathrm{B}=\mathrm{I}$ see/don't see $\beta$ ), and the intensities of either flash being input values.

These parallels could be beneficial for both psychology and physics. Thus, the cosphericity and distance tests developed in psychology (Dzhafarov \& Kujala, in press b; Kujala \& Dzhafarov 2008) could be applicable to non-Bohmian versions of EPR, for example, involving momentum and location. We see the main challenge, however, in finding a principled way to quantify and classify degrees and forms of both compliance with and violations of selectiveness of influences (or classical determinism). We only have one alternative to classical determinism in physics: quantum mechanics. This may not be enough for biological and social behavior (Dzhafarov \& Kujala, in press a).

\section{Quantum probability and cognitive modeling: Some cautions and a promising direction in modeling physics learning}

\section{doi:10.1017/S0140525X12002932}

\section{Donald R. Franceschetti and Elizabeth Gire}

\section{Department of Physics and Institute for Intelligent Systems, The University of Memphis, Memphis, TN 38152. \\ dfrncsch@memphis.edu \\ egire@memphis.edu}

\begin{abstract}
Quantum probability theory offers a viable alternative to classical probability, although there are some ambiguities inherent in transferring the quantum formalism to a less determined realm. A number of physicists are now looking at the applicability of quantum ideas to the assessment of physics learning, an area particularly suited to quantum probability ideas.

The notion that the theory of measurement and the logic of quantum probability can be applied to mental states is almost as old as quantum theory itself, and has drawn the attention of such outstanding physicists and mathematicians as Bohr (who adapted James's notion of complementarity to physics [Pais 1991]) and Penrose (1989). Pothos \& Busemeyer's (P\&B's) analysis provides a cogent statement of the case for using quantum probability. The prospect that quantum theory can be useful outside the realm of atomic and subatomic physics is, or ought to be, exciting to the community of physicists. We feel that we, as physicists with a strong interest in cognitive aspects of physics learning, can contribute to the discussion.
\end{abstract}

In its simple form, quantum theory asserts that the maximally determined states of a quantum system constitute a Hilbert space, with certain rules for the evolution of the system in time and for calculating the probabilities of various measurement outcomes. Hilbert spaces are generalizations of the ordinary two and three-dimensional vector spaces one might have encountered in high school or college. A Hilbert space is a collection of vectors with a defined inner product that allows one to determine the extent to which the vectors point in the same direction. If $\mid \psi>$ and $\mid \phi>$ are vectors in the Hilbert space, then we denote the scalar product as $\langle\boldsymbol{\varphi} \mid \psi\rangle$ using the notation introduced by Dirac (1958) and adopted by P\&B. For atomic systems, $\mid \psi>$ is a complex quantity, vector, or function having real or imaginary parts whose scalar product with itself equals one, $\langle\psi \mid \psi\rangle=1$. The probability that a measurement of a property made on the system in state $|\psi\rangle$ yields a measured value consistent with the state $|\varphi\rangle$ is given by the absolute square $|<\varphi| \psi>\left.\right|^{2}$. In strict quantum theory, once the measurement consistent with $\mid \phi>$ is made, the system is fully in the state $|\varphi\rangle$, with $\langle\varphi \mid \varphi\rangle=1$. This re-normalization process is not apparent in the analysis of the target article. It is, however, not needed if one considers only relative probabilities.

Measurable quantities in quantum theory are represented by operators that transform the vectors of the Hilbert space. Measurable quantities $\mathrm{A}$ and $\mathrm{B}$ are compatible when the operators $\mathrm{A}$ and $\mathrm{B}$ commute, that is, for all possible states $|\varphi>, \mathrm{AB}| \varphi>\mathrm{BA} \mid \varphi>$. The non-commutation of any $\mathrm{A}$ and $\mathrm{B}$ is ultimately determined by the basic lack of commutation of position and momentum of a particle, which gives rise to the uncertainty principle for the operators for position and momentum, which are conjugate operators in a technical sense (Bohr's complementarity applied to the most basic mechanical measurements). When quantum probability is applied to mental states or likelihood judgments, as is done by $\mathrm{P} \& \mathrm{~B}$, one must make a number of assumptions about the predicates "is a feminist" or "is a bank teller," which fall outside the realm of physics in which complementary attributes are easier to define.

For systems not maximally determined, that is, systems that have not passed through a sufficient number of filters to determine the values of a full set of commuting observables, a single state vector in Hilbert space cannot provide a complete description. Such systems are more generally said to be in mixed states, which are incoherent superpositions of the pure quantum states, and are described by a density operator on the Hilbert space of possible maximally specified states. In terms of probable outcomes of measurements, the mixed state allows interpolation between the realm of Bayseian probability in which $\mathrm{P}(\mathrm{AB} \mid \mathrm{A})$ is $\mathrm{P}(\mathrm{A}) \mathrm{P}(\mathrm{B} \mid \mathrm{A})$ and quantum probability, in which the Baysian assumptions absolutely do not hold for sequential measurements of incompatible measurements.

Treatment of mixed states is possible using the density matrix formalism introduced by Von Neumann in 1927, which is concisely summarized by Fano (1957). The trace of the density matrix (sum of its diagonal elements) is unity, as the system must be in some state. Multiply the density matrix by itself, however, and the trace is unity only if the system is in a pure, that is, maximally characterized, state. For systems in a mixed state, it is possible to define an entropy function that characterizes our lack of knowledge about the state. Making measurements on a system in a mixed state produces a mixed state of lower entropy or a pure state.

An important illustration of the possibilities suggested by the density matrix is provided by the work of Bao et al. (2002). In studying the mental models used by classes of undergraduates in answering a group of related physics questions, they assign a vector of unit length to each student, depending on the models used by the student, and then from a density matrix to represent the entire class. As the class evolves with instruction, the density matrix changes and approaches that for a pure state. As the students progress in their learning, the density matrix changes, as testing is a form of measurement. Ultimately, one ends up with a pure state for the well-instructed class. 
Our comments are not intended to disparage the importation of ideas from quantum probability to applications in psychology, but rather to draw attention to the richness of the formalism and some possible pitfalls. We hope that there will be continuing interaction among quantum theorists, physics teachers, and psychologists, to their mutual benefit.

\section{Does quantum uncertainty have a place in everyday applied statistics?}

\section{doi:10.1017/S0140525X12002944}

\section{Andrew Gelman and Michael Betancourt \\ Department of Statistics, Columbia University, New York, NY 10027. gelman@stat.columbia.edu \\ betanalpha@gmail.com \\ http://www.stat.columbia.edu/ gelman}

\begin{abstract}
We are sympathetic to the general ideas presented in the article by Pothos \& Busemeyer (P\&B): Heisenberg's uncertainty principle seems naturally relevant in the social and behavioral sciences, in which measurements can affect the people being studied. We propose that the best approach for developing quantum probability models in the social and behavioral sciences is not by directly using the complex probabilityamplitude formulation proposed in the article, but rather, more generally, to consider marginal probabilities that need not be averages over conditionals.
\end{abstract}

We are sympathetic to the proposal of modeling joint probabilities using a framework more general than the standard model (known as Boltzmann in physics, or Kolmogorov's laws in probability theory) by relaxing the law of conditional probability, $p$ $(x)=\Sigma_{y} p(x \mid y) p(y)$. This identity of total probability seems perfectly natural, but is violated in quantum physics, in which the act of measurement affects what is being measured, and it is well known that one cannot explain this behavior using the standard model and latent variables. (There have been some attempts to reconcile quantum physics with classical probability, but these resolutions are based on expanding the sample space so that measurement is no longer represented by conditioning, thus defeating the simplicity of the probabilistic approach.) The generalized probability theory suggested by quantum physics might very well be relevant in the social sciences.

In standard probability theory, the whole idea of conditioning is that there is a single joint distribution - parts of which may be unobserved or even unobservable, as in much of psychometrics - and that this distribution can be treated as a fixed object measurable through conditioning (e.g., the six blind men and the elephant). A theory that allows the joint distribution to change with each measurement could be appropriate for models of context in social science, such as Mischel's idea of allowing personality traits to depend on the scenario. Just as psychologists have found subadditivity and superadditivity of probabilities in many contexts, we see the potential gain of thinking about violations of the conditional probability law. Some of our own applied work involves political science and policy, often with analysis of data from opinion polls, where there are clear issues of the measurement affecting the outcome. In politics, "measurement" includes not just survey questions but also campaign advertisements, get-out-the-vote efforts, and news events.

We propose that the best way to use ideas of quantum uncertainty in applied statistics (in psychometrics and elsewhere) is not by directly using the complex probability-amplitude formulation proposed in the article, but rather by considering marginal probabilities that need not be averages over conditionals. In particular, we are skeptical of the proposed application of quantum probability to the famous "Linda example." Kahneman and Tversky's "representativeness heuristic" is to us a more compelling model of that phenomenon.

How exactly would we apply a quantum probability theory to social science? A logical first step would be to set up an experiment sensitive to the violation of the law of conditional probability: a twoslit-like model for a social statistics setting in which measurement affects the person or system being measured. Consider, for example, a political survey in which the outcome of interest, $x$, is a continuous measure of support for a candidate or political position, perhaps a 0-100 "feeling thermometer" response. An intermediate query, $y$, such as a positive or negative report on the state of the economy, plays the role of a measurement in the Heisenberg sense. The marginal distribution of support might well be different than the simple mixture of the two conditional distributions, and we would consequently expect $p(x) \neq \Sigma_{y} p(x \mid y) p(y)$.

A more sophisticated approach, and at the same time a stronger test of the need for quantum probabilities, is akin to the original Stern-Gerlach experiments. Participants would be asked a series of polarizing questions and then split by their responses. Those two groups would then be asked a further series of questions, eventually returning to the initial question. If, after that intermediate series of questions, a significant number of participants changed their answer, there would be immediate evidence of the failing of classical probabilities, and a test bed for quantum probability models.

The ultimate challenge in statistics is to solve applied problems. Standard Boltzmann/Kolmogorov probability has allowed researchers to make predictive and causal inferences in virtually every aspect of quantitative cognitive and social science, as well as to provide normative and descriptive insight into decision making. If quantum probability can do the same-and we hope it can - we expect this progress will be made as before: developing and understanding models, one application at a time.

\section{Cognitive architectures combine formal and heuristic approaches}

\section{doi:10.1017/S0140525X12002956}

\section{Cleotilde Gonzalez ${ }^{\mathrm{a}}$ and Christian Lebiere ${ }^{\mathrm{b}}$}

aSocial and Decision Sciences Department, Dynamic Decision Making Laboratory and ${ }^{\mathrm{b}}$ Psychology Department, Carnegie Mellon University, Pittsburgh, PA 15213. coty@cmu.edu cl@cmu.edu http://www.cmu.edu/ddmlab/

Abstract: Quantum probability (QP) theory provides an alternative account of empirical phenomena in decision making that classical probability $(\mathrm{CP})$ theory cannot explain. Cognitive architectures combine probabilistic mechanisms with symbolic knowledge-based representations (e.g., heuristics) to address effects that motivate QP. They provide simple and natural explanations of these phenomena based on general cognitive processes such as memory retrieval, similarity-based partial matching, and associative learning.

Pothos \& Busemeyer (P\&B) must be lauded for providing an alternative way to formalize probabilities in cognitive models in a world where classical probability $(\mathrm{CP})$ theory dominates modeling. The findings that they discuss are indeed a challenge for CP. Existing heuristic explanations are often unsatisfactory, offering few detailed quantitative explanations of the cognitive processes involved. For example, how do heuristics emerge and how do they relate to a formal representation of psychological processes? $\mathrm{P} \& \mathrm{~B}$ demonstrate how quantum probability $(\mathrm{QP})$ theory addresses these challenges.

Here, we argue that cognitive architectures, a modeling approach with a long history in the cognitive sciences, may also address the outlined challenges. The main article contained little discussion of cognitive architectures, with the few examples showcasing ones that rely heavily on CP. Whereas cognitive architectures do have a probabilistic aspect with stochastic components to processes such as memory retrieval or action selection, they combine probabilistic processing (i.e., CP) with symbolic knowledge-based representations (e.g., heuristics). 


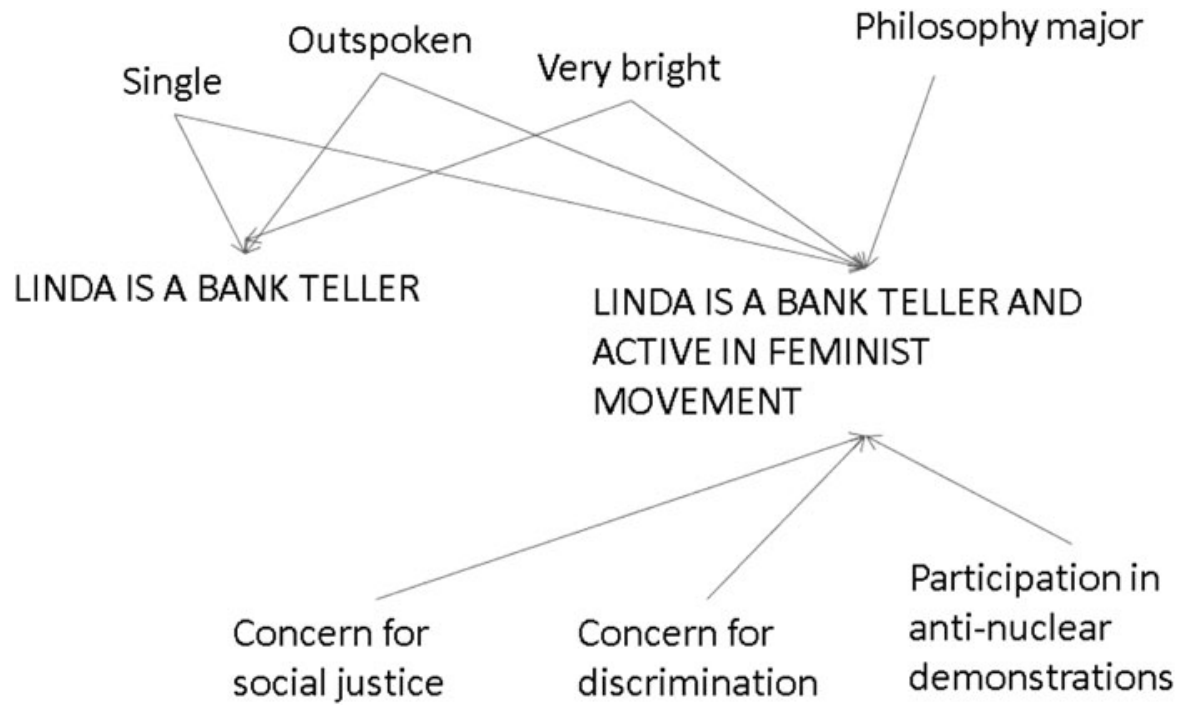

Figure 1 (Gonzalez \& Lebiere). Attributes and associations to the options in the Linda problem.

Cognitive architectures are computational implementations of cognitive theories that unify and represent a full range of human cognitive processes from perception to action (Newell 1990). Their strengths are derived from a tight integration of their different components, particularly those satisfying the functional constraints to help maintain the "big picture" needed to understand the human mind (Anderson \& Lebiere 2003). Performance in any given task is a result of the complex interactions between various modules, their underlying mechanisms, and the resulting information flows. Adaptive Control of ThoughtRational (ACT-R) (Anderson 2007; Anderson \& Lebiere 1998) is one of the most well-known architectures, with hundreds of published models representing a broad range of tasks and phenomena. ${ }^{1}$ Its distinguishing feature is the ability to combine symbolic representations (declarative chunks and procedural rules) with subsymbolic processes tuned by statistical learning. This allows ACT-R to create symbolic representations of heuristics and knowledge in the form of production rules and declarative chunks with probabilistic processes that can capture many mechanics of CP and even QP. In fact, there are researchers who have taken up the task of explaining a portion of the large collection of cognitive biases and heuristics through memory processes. These studies often use ACT-R cognitive models (Marewski \& Mehlhorn 2011; Marewski \& Schooler 2011; Schooler \& Hertwig 2005).

Although these are commendable efforts, the large variety of cognitive biases cannot be all explained by one single comprehensive model. Rather, these researchers offer multiple models: one for each type of heuristic (Marewski \& Mehlhorn 2011). We have made a similar observation regarding models of decisions from experience, which are often task-specific and are developed to account for only one variation of a given task (Gonzalez \& Dutt 2011; Lejarraga et al. 2012). Therefore, a modeling methodology that provides a unified approach to a variety of tasks by leveraging the same architectural mechanisms is needed. For example, an instance-based learning (IBL) model of repeated binary choice offers a broad and robust unified explanation of human behavior across multiple paradigms and variations of these tasks (Gonzalez 2013; Gonzalez \& Dutt 2011; Lejarraga et al. 2012).

IBL models are process models that rely on ACT-R learning and memory mechanisms, most notably those involving declarative representations and the activation mechanisms. Activation is a value corresponding to each chunk in memory, learned to reflect its usage statistics. It controls information retrieval from memory and can be interpreted as the log odds of retrieval. The activation mechanisms involve four main processes: base-level learning that accounts for recency and frequency of information, similaritybased partial matching that represents the mismatches between a task's situational attributes and those stored in memory, associative learning that reflects the impact of contextual values and the associations between cues in different contexts, and a stochastic component that makes the retrieval process probabilistic.

As an example, consider the Linda problem used by $\mathrm{P} \& \mathrm{~B}$. The conjunction fallacy is explained by IBL models as follows. Two main options (i.e., instances) are considered in the decision process: (1) Linda as a bank teller, and (2) Linda as a bank teller and a feminist. Whereas a formal probability theory such as QP and CP represent the latter as a logical conjunction, they are represented as independent instances in memory in a computational theory such as ACT-R. The activation of these two instances is determined by the attributes in the problem's description, and in the memory relationships to "bank teller" and to "feminist," as is captured by the associative learning mechanism governing the spreading of activation. A scenario of the described attributes and their possible associations to the two options is presented in Figure 1. Without going into detail regarding the activation processes, the figure shows that the number of attributes associated with option 1 (3) is less than the number of associations (7) related to option 2 . Therefore, option 2 has a higher probability of retrieval given a higher activation, and it would be selected more often. Although this is an impossibility according to CP, our example illustrates how IBL models and ACT-R more generally account for this effect, and explains it in terms of simple memory processes. When eschewing a formal probabilistic framework in favor of a computational account, apparent impossibilities simply dissolve in light of the cognitive processes used to actually produce the decisions.

In general, we believe that the advantages of QP over CP can all be addressed through the mechanisms in ACT-R models. Superposition, for example, can be addressed through the similarity metrics used in the partial matching mechanism in the activation equation, entanglement can be addressed through the contextual effects of associative memory, and incompatibility can be addressed through the recency effects in the base-level learning processes.

In conclusion, although we agree with $\mathrm{P} \& \mathrm{~B}$ on the large body of evidence that goes directly against CP principles, we do not believe that an alternative probabilistic theory is essential to understanding the intuition behind human judgment in such conditions. Cognitive architectures are able to address these challenges in a natural way that leverages the characteristics of general cognitive processes such as memory retrieval.

\section{NOTE}

1. ACT-R code, publications, and models are available at http://act-r. psy.cmu.edu. 
Commentary/Pothos \& Busemeyer: Can quantum probability provide a new direction for cognitive modeling?

\section{Quantum probability and comparative cognition}

\section{doi:10.1017/S0140525X12002968}

\section{Randolph C. Grace and Simon Kemp \\ Department of Psychology, University of Canterbury, Private Bag 4800, Christchurch, New Zealand. \\ randolph.grace@canterbury.ac.nz \\ simon.kemp@canterbury.ac.nz}

\begin{abstract}
Evolution would favor organisms that can make recurrent decisions in accordance with classical probability $(\mathrm{CP})$ theory, because such choices would be optimal in the long run. This is illustrated by the base-rate fallacy and probability matching, where nonhumans choose optimally but humans do not. Quantum probability $(\mathrm{QP})$ theory may be able to account for these species differences in terms of orthogonal versus nonorthogonal representations.
\end{abstract}

Pothos \& Busemeyer (P\&B) are to be congratulated for their target article, which presents an exciting new paradigm for modeling cognition. We suggest that by including a comparative perspective grounded in scientific realism, quantum probability (QP) theory may have even more promise as a framework for describing cognition in humans and nonhumans alike. Moreover, from this view, QP and classical probability $(\mathrm{CP})$ theory are viewed as complementary, not contradictory, frameworks.

Shepard (1994) argued that organisms that evolved in a physical world would likely have internalized the constraints of that world. If QP theory provides the formal basis for the physical description of the world, then it should also provide the formal basis for the description of cognitive processes within organisms that evolved in that world. (It is interesting to consider why quantum mechanics is hard to understand, but we leave that for another day). However, P\&B seem tempted to abandon scientific realism - prematurely, in our view. In the context of discussing the implications of QP for human rationality, they write: "The main problem with classical optimality is that it assumes a measurable, objective reality," and later, "Quantum theory assumes no measurable objective reality" (sect 5). It is understandable that $\mathrm{P} \& \mathrm{~B}$ draw clear distinctions between $\mathrm{CP}$ and QP models, but there is a risk of losing sight of their complementary aspect.

If, as most believe, CP describes the workings of chance in the real world, evolution would favor organisms that are able to make decisions in accordance with CP principles, particularly in situations in which recurrent choices are possible, because such decisions would produce the best outcome in the long run. There is considerable evidence for the utility of optimality models in describing nonhuman behavior, both in the laboratory and natural ecology (e.g., optimal foraging theory [Charnov 1976; Koselj et al. 2011; Stephens \& Krebs 1986]; ideal free distribution [Krivan et al. 2008; Sebastian-Gonzalez et al. 2010]; behavioral economics [Hursh 1984; Rachlin et al. 1976]). However, it is important to acknowledge that some theorists have proposed evolutionary accounts of apparently "irrational" human behavior. For example, De Fraja (2009) produced a plausible account of why conspicuous consumption might be evolutionarily adaptive, and Kenrick et al. (2009) argue for the modularity of utility across different domains (see also Robson \& Samuelson 2011).

Notably, there are situations in which nonhumans behave optimally and consistent with CP, whereas humans do not. A prominent example is the base-rate fallacy, associated with Tversky and Kahneman's (1980) "taxi cab" problem. Here subjects are asked to estimate the probability that a taxi involved in a hitand-run accident was blue or green. They are told that $85 \%$ of the cabs in the city are green, but that a witness identified the cab as blue, whose accuracy was later found to be $80 \%$ under comparable conditions. Typically humans estimate the probability of the cab being blue as greater than $50 \%$, thus placing too much weight on the witness testimony; the correct answer (from Bayes' theorem) is $41 \%$. By contrast, nonhumans (pigeons) did

not neglect base rates and responded optimally when tested in an analogue task (Hartl \& Fantino 1996). Another example in which humans fail to respond optimally but nonhumans do not is probability matching (Fantino \& Esfandiari 2002).

These species differences can be accommodated within QP theory by assuming that nonhumans construct orthogonal representations in situations with multiple sources of information, leading to behavior consistent with CP and optimality, whereas humans do not. This corresponds to the distinction between "compatible" and "incompatible" questions in the target article. From a comparative perspective, the question is why and under what conditions would humans be more likely to construct nonorthogonal representations ("incompatible questions").

Comparative research on visual categorization suggests that differences in attention-related processes may provide a clue. In the randomization procedure, subjects view circular discs with sine-wave gratings that vary in frequency and orientation, and assign each to one of two categories (Ashby \& Gott 1988; Maddox et al. 2003). Two types of tasks are commonly used: one in which category exemplars are defined in terms of differences along one dimension, whereas the other dimension is irrelevant, and one in which both dimensions are relevant. The former is termed a "rule-based" (RB) task because accurate performance can be described by a verbal rule, whereas the latter is known as "information integration" (II) because correct responding depends on both dimensions. With training, humans can learn to respond correctly on the II task but when debriefed cannot describe their performance in terms of verbal rule, and typically say that they responded on the basis of a "gut feeling" (Ashby \& Maddox 2005).

The RB task is one in which selective attention facilitates performance, whereas the II task requires divided attention. Humans and nonhuman primates are able to learn the RB task faster and to a higher degree of asymptotic accuracy than the II task (Smith et al. 2010). In contrast, pigeons show no difference in learning rate or accuracy (Smith et al. 2011). Smith et al. (2012) have argued that these species differences suggest that the primate lineage may have evolved an increased capacity for selective attention. In terms of QP theory, selective attention may facilitate a shift in task representation. Pigeons may be constrained to use an orthogonal representation, in which both dimensions are given equal weight, leading to similar performance on both tasks, whereas the representation used by human and nonhuman primates is more flexible and allows for faster and more accurate learning in the $\mathrm{RB}$ task.

We agree that QP theory is an exciting new direction for modeling of human cognition, but as the previous examples suggest, it may benefit from a comparative perspective. An enduring controversy in comparative cognition has been the question of differences in intelligence across species (Macphail 1987), including whether human and nonhuman differences should be understood in qualitative or quantitative terms (Roth \& Dicke 2005). We are intrigued by the possibility that QP theory may ultimately help to resolve this question.

\section{Quantum mathematical cognition requires quantum brain biology: The "Orch OR" theory}

\section{doi:10.1017/S0140525X1200297X}

\section{Stuart R. Hameroff}

Departments of Anesthesiology and Psychology, The University of Arizona, University of Arizona Medical Center, Tucson, AZ 85724.

hameroff@u.arizona.edu

www.quantum-mind.org

Abstract: The "Orch OR" theory suggests that quantum computations in brain neuronal dendritic-somatic microtubules regulate axonal firings to 
Commentary/Pothos \& Busemeyer: Can quantum probability provide a new direction for cognitive modeling?

control conscious behavior. Within microtubule subunit proteins, collective dipoles in arrays of contiguous amino acid electron clouds enable "quantum channels" suitable for topological dipole "qubits" able to physically represent cognitive values, for example, those portrayed by Pothos \& Busemeyer (P\&B) as projections in abstract Hilbert space.

Pothos \& Busemeyer $(\mathrm{P} \& \mathrm{~B})$ suggest that human cognition can follow rules of quantum, rather than classical (Bayesian) probability mathematics. P\&B are agnostic on whether such cognition involves actual quantum activity, but quantum mathematical cognition logically requires quantum biology. Could functional quantum biology occur in the brain?

The Penrose-Hameroff "Orch OR" theory suggests that cytoskeletal microtubules inside brain neurons perform quantum computations that mediate conscious perceptions and control conscious behavior (Hameroff 1998; 2006a; 2007; 2012; Hameroff \& Penrose 1996a; 1996b; Penrose \& Hameroff 1995; 2011). In Orch OR, synaptic inputs "orchestrate" quantum superpositioned states (quantum bits, or "qubits," existing simultaneously in alternative states) in "tubulin" subunit proteins in microtubules in dendrites and soma/cell bodies of "integrate-and-fire" brain neurons (Fig. 1), microtubules in which memory may be encoded (Craddock et al. 2012b). Dendritic-somatic tubulin qubits entangle, interfere, and compute according to the Schrödinger equation during integration phases, then spontaneously reduce, or collapse to classical tubulin states that influence membrane polarization and regulate axonal firings, controlling behavior. (The Orch OR neuron is the "Hodgkin-Huxley" neuron with finer scale, deeper-order microtubule quantum influences.) At the end of each integration phase, quantum state reduction occurs by an objective threshold (Penrose "objective reduction," "OR," hence "orchestrated objective reduction": "Orch OR") for example, every $25 \mathrm{~ms}$, coinciding with " $40 \mathrm{~Hz}$ ” gamma synchrony electro-encephalography ("EEG”). Orch OR is testable, feasible, and, although criticized (McKemmish et al. 2009; Tegmark 2000; c.f. Hagan et al. 2001; Penrose \& Hameroff 2011), is increasingly supported by experimental evidence from quantum biology (Engel et al. 2007; Gauger et al. 2011; Sarovar et al. 2010; Scholes 2010).

P\&B take an extremely important step, but they omit four important features, the first being consciousness. Quantum probability and quantum biology (e.g., Orch OR) may specifically govern conscious cognition, whereas classical (Bayesian) probability and biology govern unconscious cognition (e.g., Hameroff 2010).

The second omission is information representation in the brain. In a bold and novel assertion, $\mathrm{P} \& \mathrm{~B}$ introduce projection rays in abstract Hilbert space to represent cognitive values, for example, feelings, or subjective "qualia," such as "happy" and "unhappy." Could abstract quantum projections correlate with actual quantum physical processes in brain biology? Are there qubits in the brain?

According to Orch OR, collective van der Waals London force dipoles among electron clouds of amino acid phenyl and indole rings aligned in non-polar "quantum channels" within each tubulin can orient in one direction or another, and exist in quantum superposition of both directions, acting as bits and qubits (Fig. 2a-d). Intra-tubulin channels appear to link to those in neighboring tubulins to form helical quantum channels extending through microtubules (Fig. 3a). Anesthetic gas molecules that selectively erase consciousness (sparing unconscious brain activity) bind in these channels (Craddock et al. 2012a; Hameroff 2006b). Collective dipole qubits in microtubule quantum channels can represent cognitive values, for example, P\&B's "happy/unhappy" and "job/no job" (Fig. 3b).

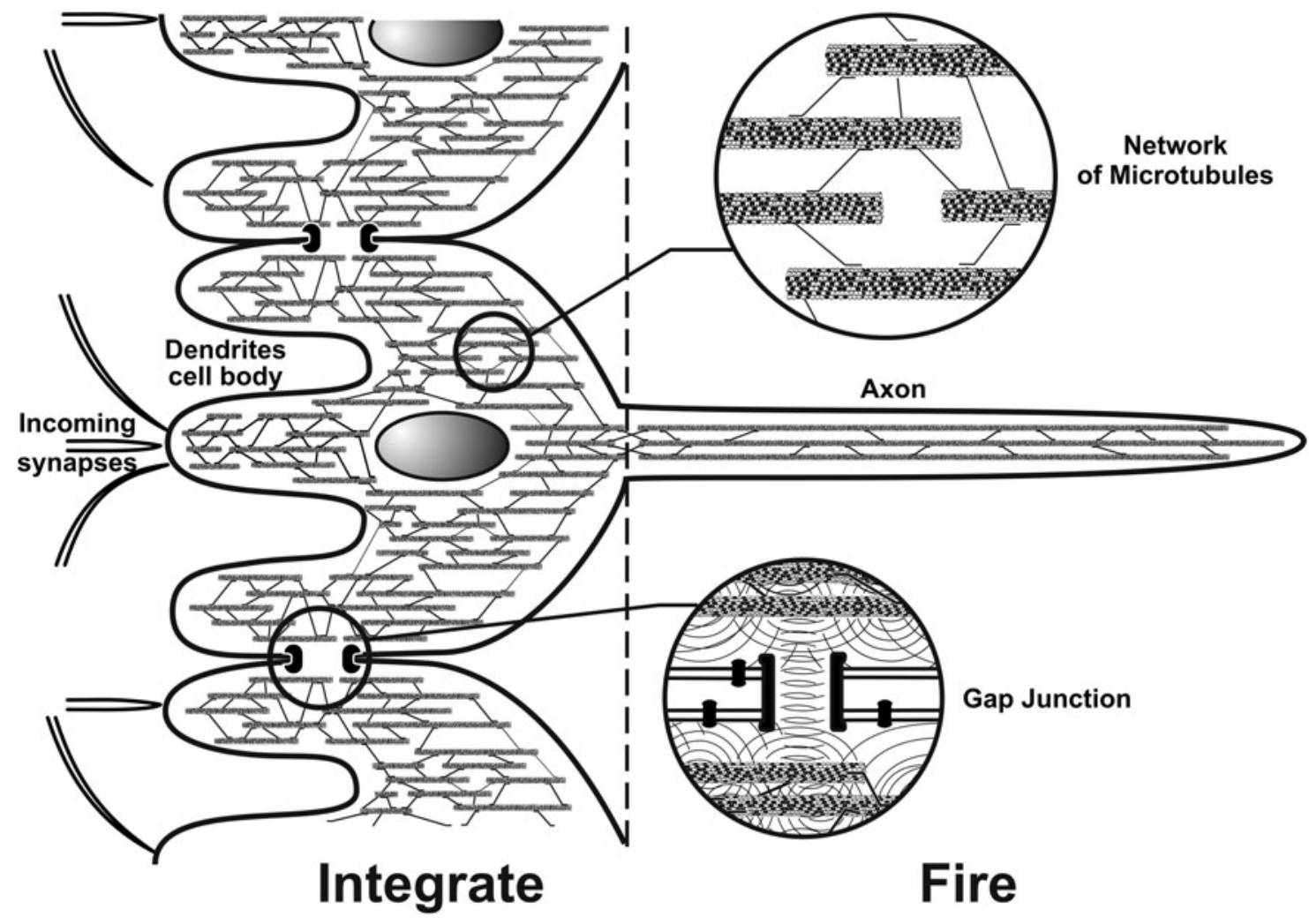

Figure 1 (Hameroff). A neuron, and portions of other neurons are shown schematically with internal microtubules. In dendrites and cell body/soma (left), microtubules are interrupted and of mixed polarity, interconnected by microtubule-associated proteins (MAPs) in recursive networks (upper circle, right). Microtubules in axons are unipolar and continuous. Gap junctions synchronize dendritic membranes, and may enable entanglement among microtubules in adjacent neurons (lower circle right). In Orch OR, microtubule quantum computations occur during dendritic/somatic integration, and the selected results regulate axonal firings. 
Commentary/Pothos \& Busemeyer: Can quantum probability provide a new direction for cognitive modeling?

(a)

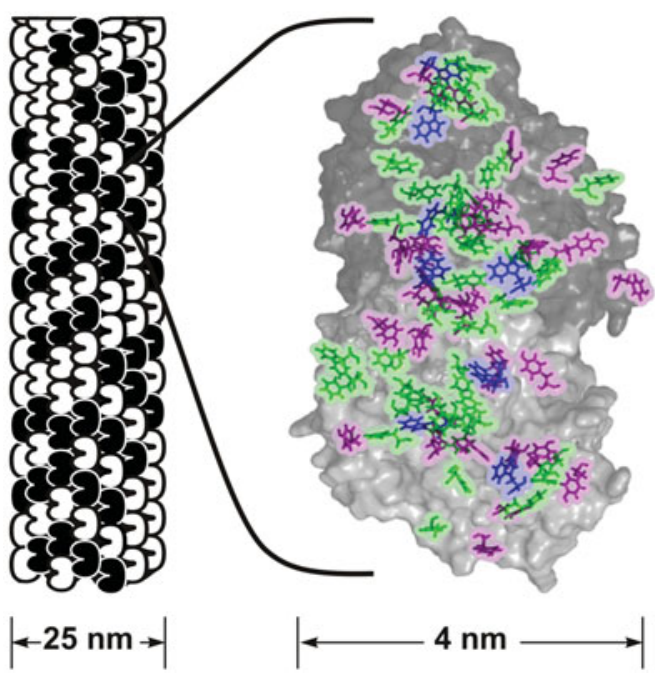

(c)
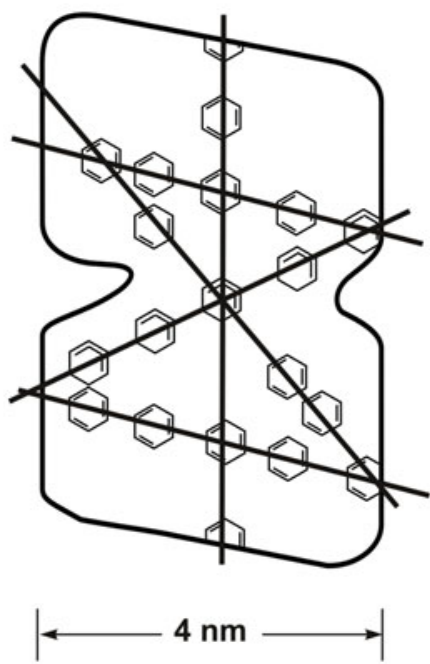

(d)
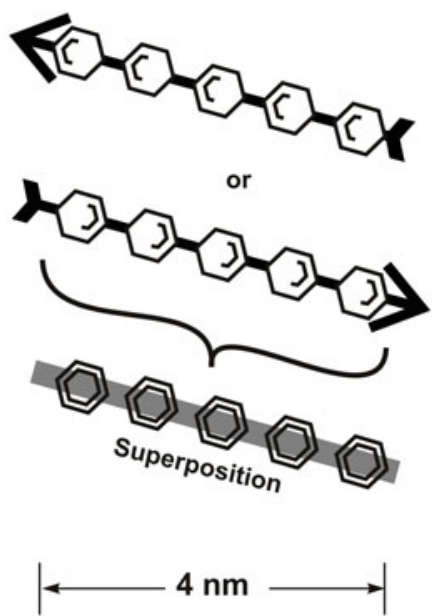

Figure 2 (Hameroff). a. A microtubule (A-lattice) is composed of peanut-shaped tubulin proteins. b. Within the microtubule, an individual tubulin shows internal (colored) van der Waals radii of aromatic amino acids tryptophan (blue), phenylalanine (purple), and tyrosine (green) traversing the protein (image provided with permission from Travis Craddock). c. Schematic tubulin illustrates aromatic rings (phenyl shown here, although indole rings are also involved) arrayed through tubulin along "quantum channels." d. Collective dipoles along such channels may exist in alternative orientations, or quantum superposition of both orientations - that is, quantum bits or "qubits."

Collective dipoles in helical quantum channels appear suitable for qubits, or "braids" in topological quantum computing in which specific pathways, rather than individual subunit states, are fundamental information units, mediated by fractional charge "anyons" (Collins 2006; Freedman et al. 2002; Kitaev 2003). Topological qubits (or comparable "adiabatic" qubits) are inherently stable, resistant to decoherence, and consistent with Orch OR (Hameroff et al. 2002; Penrose \& Hameroff 2011).
P\&B’s third omission is quantum computation. They cite three quantum features: (1) superposition (co-existing possibilities), (2) entanglement (connectedness of separated states), and (3) noncommutativity (context-dependence, "incompatibility"). The three features together comprise quantum computation in which superpositioned "qubits" interfere and compute by entanglement, and then reduce/collapse to definite output states. In a series of quantum computations, output of each reduction (a)

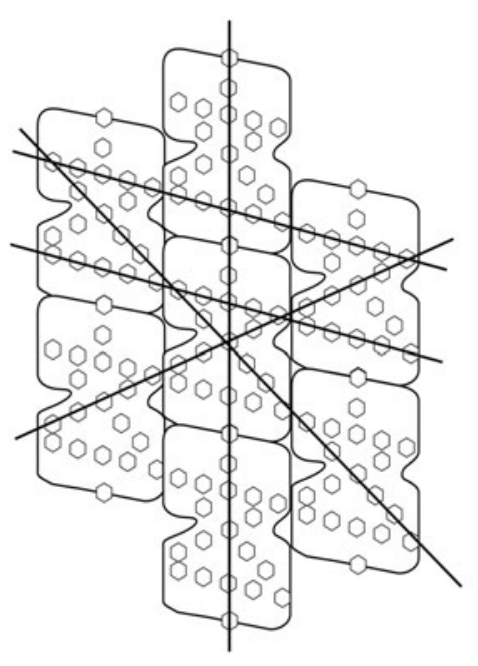

(b)

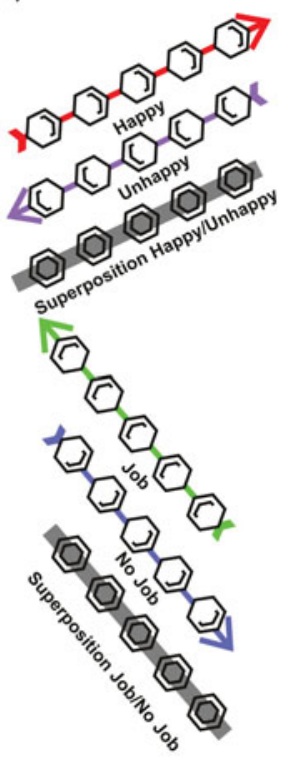

(c)

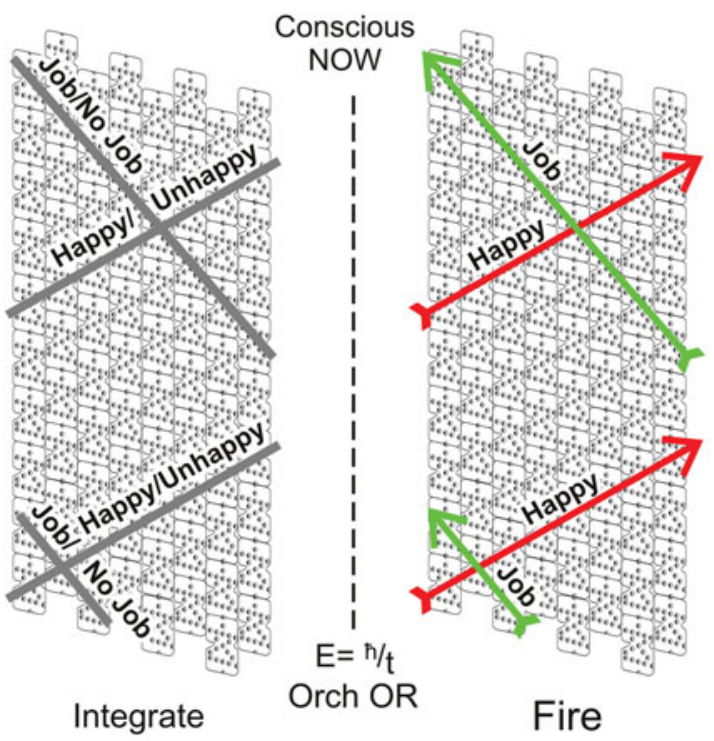

Figure 3 (Hameroff). a. Seven tubulins in a microtubule A-lattice neighborhood are shown in which contiguous arrays of aromatic amino acid rings traverse tubulin and align with those in neighboring tubulins, resulting in helical "quantum channel" pathways throughout the microtubule. b. Dipole orientations in two different helical pathways along quantum channels represent alternate cognitive values and their superpositions (as described by $\mathrm{P} \& \mathrm{~B}$, "happy/unhappy," "job/no job") - that is, topological dipole qubits. c. Superpositioned qubits in (a larger portion of) a single A-lattice microtubule are shown during the integration phase, prior to reduction/self-collapse by the Orch OR mechanism. d. Post-reduction values selected in the Orch OR process are illustrated, a moment of conscious awareness having occurred. 
Commentary/Pothos \& Busemeyer: Can quantum probability provide a new direction for cognitive modeling?

provides input for the next, hence context-dependent incompatibility. P\&B's three features add up to cognitive quantum computation, as suggested in Orch OR.

The fourth omission, (and essence of the "measurement problem" in quantum mechanics) is reduction of quantum superpositions to classical output states ("collapse of the wave function"), what $\mathrm{P} \& \mathrm{~B}$ refer to as "disambiguating a superposition state." There are numerous theoretical suggestions as to how and why superpositions reduce to classical states (multiple worlds, decoherence, observer effect), but none address the nature of superposition, and each is unsatisfactory for various reasons (e.g., the von Neumann/Wigner "observer effect" puts conscious observation outside science). Addressing this problem, Penrose (1989; 1994; 1996; 2004) proposed (1) superpositions were actual separations in underlying spacetime geometry, the fine structure of the universe, (2) such separations were unstable, and reduce to definite states after time $t$ by an objective threshold given by the indeterminacy principle $E=\hbar / t$ (where $E$ is the magnitude of the superposition/separation and $\hbar$ is Planck's constant over $2 \pi$ ), and (3) such events result in moments of conscious experience and choice. Orch OR is the neurobiological framework for Penrose OR, and perfect solution for P\&B's required "constructive role for the process of disambiguating a superposition state."

Figure 3c shows two superpositioned dipole pathways acting as topological qubits in a microtubule A-lattice and representing P\&B’s "happy/unhappy” and “job/no job” (perhaps more properly "job satisfaction" and "job dissatisfaction"). The qubits are coupled; for example, "happy" and "job" are more likely to coincide than "happy" and "no job". During the integration phase, superpositioned qubits entangle, evolve, and compute (in a microtubule memory bed) by the Schrödinger equation until threshold by $E=\hbar / t$ is met, OR occurs, and classical microtubule states are selected (correlating with "happy" and "job" in the example given). According to Orch OR, consciousness occurs in the end-integration moment of reduction, and microtubule states selected in the OR process govern axonal firing and behavior (Hameroff 2012).

If cognition (and consciousness) utilize quantum mathematics, entanglement, and non-commutativity, as $\mathrm{P} \& \mathrm{~B}$ suggest, the brain is likely to be using quantum computation, such as described in Orch OR. From a well-known example of inductive reasoning: "If the brain swims, looks, and quacks like a (quantum) duck, then it probably is a (quantum) duck."

\section{ACKNOWLEDGMENTS}

I thank Dave Cantrell for artwork, Travis Craddock for Figure 2b, Hartmut Neven and Saatviki Gupta for comments, and Sir Roger Penrose for collaboration.

\section{Quantum probability and conceptual combination in conjunctions}

\author{
doi:10.1017/S0140525X12002981
}

\section{James A. Hampton \\ Department of Psychology, City University London, City University, Northampton Square, London EC1V OHB, United Kingdom. Hampton@city.ac.uk \\ www.staff.city.ac.uk/hampton}

Abstract: I consider the general problem of category conjunctions in the light of Pothos \& Busemeyer (P\&B)'s quantum probability (QP) account of the conjunction fallacy. I argue that their account as presented cannot capture the "guppy effect" - the case in which a class is a better member of a conjunction $\mathrm{A}^{\wedge} \mathrm{B}$ than it is of either $\mathrm{A}$ or $\mathrm{B}$ alone.

My commentary will focus on a specific point in the target article, relating to the formation of conjunctions, either of subjective probabilities or of degrees of category membership.
Consider people's tendency to overestimate conjunctive probabilities in the famous Linda case (Tversky \& Kahneman 1983). The QP model (P\&B, Fig. 2) explains the conjunction fallacy as follows. By projecting the initial state vector (Linda) first onto the "feminist" vector, the angle with the "bank teller" vector is thus reduced, and, therefore, the projection to "bank teller" via "feminist" is stronger than the direct projection straight to "bank teller." To explain this effect psychologically, they then offer the following suggestion: "once participants think of Linda in more general terms as a feminist, they are more able to appreciate that feminists can have all sorts of professions, including being bank tellers." In some way, Linda is more easily imaginedperhaps she seems more real - so that being considered a feminist makes it more likely that she might also be a bank teller.

This is a totally novel explanation for this effect. Remember that the initial story about Linda (that she was a liberal in college) makes it very likely that she is a feminist. It is unclear why consideration of her being a feminist should then increase the subjective likelihood of her having an improbable job for a graduate such as being a bank teller. This explanation bears no relation to the traditional use of representativeness to account for the conjunction fallacy.

The conjunction fallacy bears close resemblance to another phenomenon, also mentioned by P\&B: the overextension of category conjunctions (Hampton 1987; 1988b). For example, people are more likely to categorize chess as a "sport that is a game" than as simply a "sport," in much the same way that they judge Linda as more likely to be a feminist bank teller than simply a bank teller. The effect occurs across a range of different categories (for example a ski-lift is more likely to be considered a "machine that is a vehicle" than it is to be considered a vehicle, or a refrigerator is more likely to be a "household appliance that is furniture" than it is to be simply "furniture.")

The composite prototype model (Hampton 1987; 1988b) developed to handle such cases proposes that the two conjuncts (for example, feminist and bank teller) are composed into a single composite concept, by merging their intensions (their associated descriptive properties). Therefore, a sport that is a game will have properties inherited from sports (competitive, skilled, and physical) and also from games (rules and scoring). Further elaboration of the representation may then take place to improve the coherence of the new composite (Hampton 1997). In terms of Figure 2 in P\&B's article, a new vector would be formed for the conjunction that roughly bisects the "feminist" and "bank teller" vectors, as in Figure 1 here. As a consequence, provided the

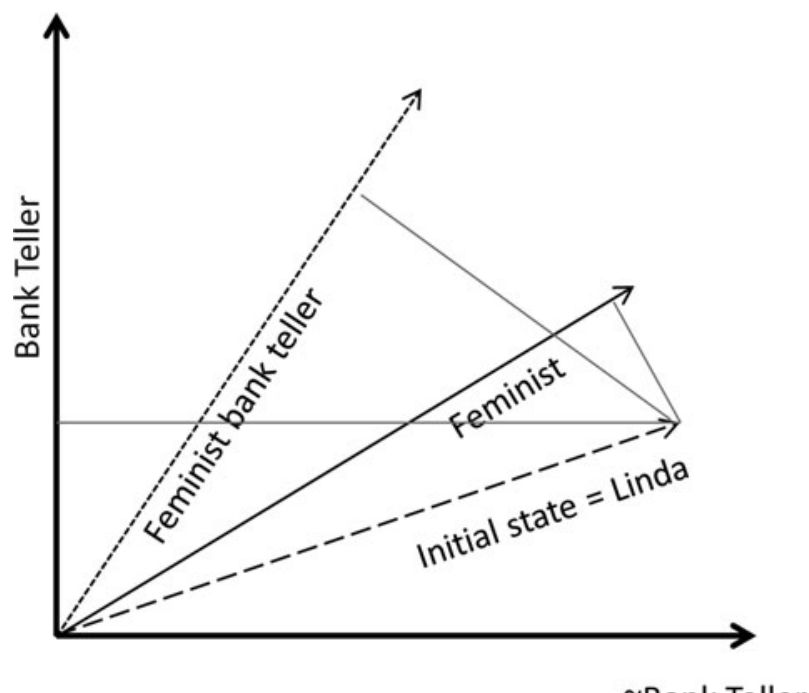

Figure 1 (Hampton). Construction of a new vector for feminist bank teller as intermediate between the two conjuncts. 
Commentary/Pothos \& Busemeyer: Can quantum probability provide a new direction for cognitive modeling?

initial state vector lies outside the angle formed by the two constituent concepts (as here), the projection of the initial state vector onto the composite (conjunction) vector will always lie between the projections on the two other vectors.

In psychological terms, Linda has many properties in favour of her being a feminist, and hardly any in favour of her being a bank teller; therefore, she has a moderate number of properties suggesting that she is a feminist bank teller. The two degrees of similarity are averaged. According to the representativeness heuristic, subjective probability is assessed through similarity (number of relevant properties). Although the mechanism proposed by the representativeness heuristic is very different from that proposed by $\mathrm{P} \& \mathrm{~B}$, so far the predictions are similar. In terms of subjective likelihood, Linda should most likely be a feminist, next a feminist bank teller, and least likely a bank teller.

However, the two accounts can be dissociated. A critical test for the QP account concerns the so-called "guppy effect." In categorization tasks, people will sometimes claim that something is a better example of a conjunction than of either of the conjuncts. In their famous critique of the application of fuzzy logic to conceptual combination, Osherson and Smith (1981) pointed out that guppies could be considered better pet fish than they were either pets or fish. The account offered by $\mathrm{P} \& \mathrm{~B}$ for conjunctions will have trouble with this result. If the initial state vector must first be projected to one vector and then onto the second, the probability must always be reduced each time (see Fig. 2).

Therefore, it is not possible within this model to obtain this double overextension of conjunctions. In contrast, the intensional account offered by the composite prototype model does allow that an example can be more similar to the composite than to either conjunct. Pet fish lack some key properties of pets (they are not warm and affectionate) and also lack typical properties of fish (they are not caught in nets and fried with breadcrumbs). It is therefore quite feasible for an exemplar such as the guppy, which also lacks exactly these properties, to be a better fit to the conjunction than to either conjunct. Although much rarer than simple overextension of one conjunct, empirical data do in fact support the guppy effect (Storms et al. 2005.) A better vector representation would therefore be that shown in Figure 3, where a new vector is created to represent the composite prototype of a pet fish, and the guppy projects more strongly

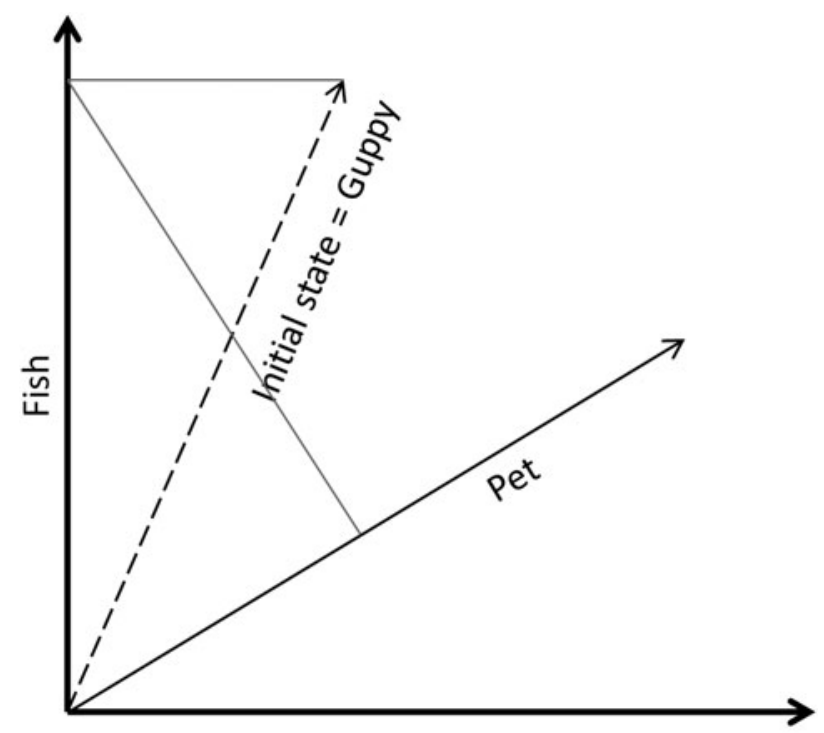

$\sim$ Fish

Figure 2 (Hampton). The P\&B projection from guppy to fish, and then to pet. The conjunction can never be greater than both conjuncts.

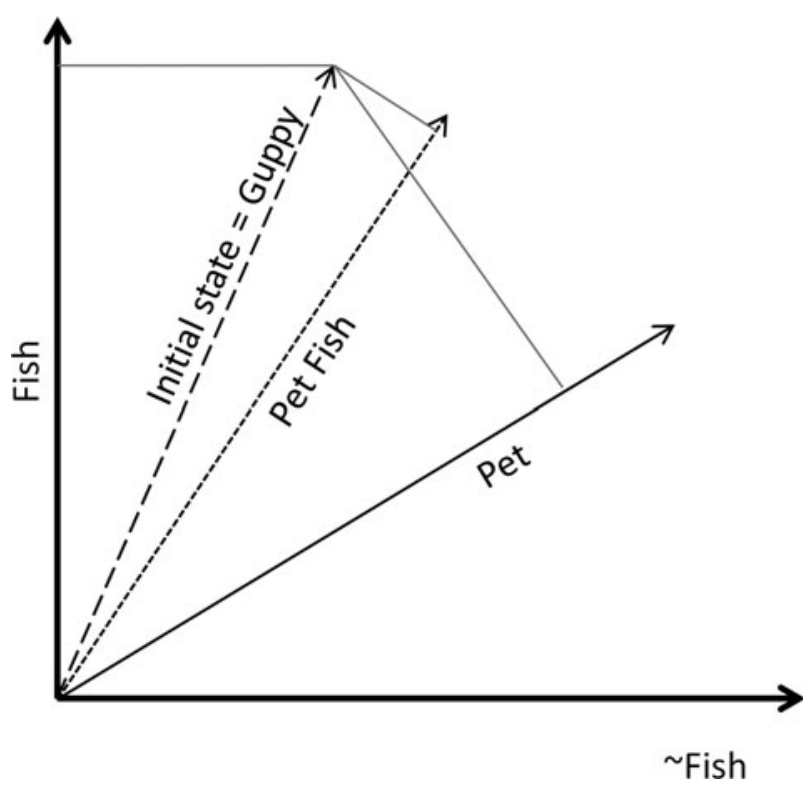

Figure 3 (Hampton). Projection from guppy to a new vector representing the combination pet fish allows it to be a better pet fish than it is either a pet or a fish.

onto this conjunctive vector than it does onto either the pet or the fish vectors.

In conclusion, as a psychological account of conceptual combination, QP appears to lack sufficient resources to handle the intensional reasoning that can be seen to characterize much of human thought (Hampton 2012). The notion of concepts as regions of subspace makes good sense, as it captures the vagueness and context-dependence of how concepts enter thoughts. However, the simple projection mechanism suggested in Figure 2 is unlikely to provide a rich enough framework to capture the ways in which concepts combine.

\section{Is quantum probability rational?}

\author{
doi:10.1017/S0140525X12002993
}

\section{Alasdair I. Houston ${ }^{a}$ and Karoline Wiesner ${ }^{b}$ \\ ${ }^{a}$ School of Biological Sciences, University of Bristol, Bristol BS8 1UG, United

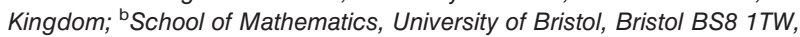 United Kingdom. \\ a.i.houston@bristol.ac.uk \\ k.wiesner@bristol.ac.uk \\ http://www.bristol.ac.uk/biology/people/alasdair-i-houston/overview.html http://www.maths.bristol.ac.uk/ enxkw/}

\begin{abstract}
We concentrate on two aspects of the article by Pothos \& Busemeyer $(\mathrm{P} \& \mathrm{~B})$ : the relationship between classical and quantum probability and quantum probability as a basis for rational decisions. We argue that the mathematical relationship between classical and quantum probability is not quite what the authors claim. Furthermore, it might be premature to regard quantum probability as the best practical rational scheme for decision making.
\end{abstract}

Pothos \& Busemeyer ( $\mathrm{P} \& \mathrm{~B})$ provide an intriguing overview of quantum probability as a framework for modeling decision making. In this commentary, we concentrate on two aspects of their article: the relationship between classical probability (CP) and quantum probability $(\mathrm{QP})$ and its implications for ideas about rationality in humans and other animals.

In order to evaluate the contribution that QP makes to our understanding of cognitive processes, it is necessary to be clear about the relationship between CP and QP. In Section 4.3, 
Commentary/Pothos \& Busemeyer: Can quantum probability provide a new direction for cognitive modeling?

$\mathrm{P} \& \mathrm{~B}$ reject the view that $\mathrm{QP}$ provides a better account of human decision making "simply because it is more flexible." We are not completely happy with some of the statements that $\mathrm{P} \& \mathrm{~B}$ make about this issue. From a mathematical point of view, CP is embedded as a special case in the more general non-commutative (also referred to as "quantum") probability theory. There are no cases of CP distributions and the evolution thereof that cannot be represented in a quantum probabilistic framework. Once the Heisenberg matrix mechanics is used (equivalent to the Schrödinger wave vector notation used by $\mathrm{P} \& \mathrm{~B}$ ) it can be shown that any commutative quantum theory can be written as a CP theory, and vice versa, $\mathrm{CP}$ theory can be written as a commutative quantum theory. Hence, CP is strictly a special case of non-commutative (quantum) probability (see Streater 2000). To clarify the connection to P\&B by example: what they call the "law" of double stochasticity is merely a special case in quantum theory, that of projective measurement, which is a subset of all (generalised) measurements.

The discussion about "QP theory violating the law of total probability" would be helped by clarifying the law of total probability. It states that the probabilities of all elements in a set sum up to the probability of the whole set. In quantum mechanics the whole can be less than the sum of its parts because of superposition of states. An equation such as the one in the target article's appendix

$$
\begin{aligned}
& \text { Prob(happy, unknown employment) } \\
& \quad=\| M \cdot U(t) \cdot \frac{1}{\sqrt{2}}\left|\psi_{\text {employed }}\right\rangle+M \cdot U(t) \cdot \frac{1}{\sqrt{2}}\left|\psi_{\sim \text { employed }}\right\rangle \|^{2}
\end{aligned}
$$

is more helpful if stripped down to the "superposition part" (the state is "separable"), that is, the only information that matters is

$$
\begin{aligned}
& \text { Prob(unknown employment }) \neq \operatorname{Prob}(\text { not employed }) \\
& \quad+\operatorname{Prob}(\text { employed })
\end{aligned}
$$

Our final topic is the relationship between rational decision making and natural selection. In this context, it is worth noting that the decisions of nonhuman animals violate the principles of rational decision making (Houston et al. 2007b). Given that natural selection is expected to favour optimal choices, there is a tension between natural selection and the observed behaviour of animals. One response is to argue that animals only need to make approximately optimal decisions in particular circumstances - the circumstances in which they have evolved. This idea is related to the suggestion that decision making is based on heuristics, as mentioned by $\mathrm{P} \& \mathrm{~B}$ in Section 4.2. A more interesting possibility is that what appear to be suboptimal choices are part of an optimal strategy for an environment that is richer than was originally envisaged. For example, Houston et al. (2007a) show that apparent violations of transitivity can be generated by the optimal state dependent strategy given that the options presented to the animal will persist into the future. In essence, the violations appear because the experimenter does not have the same view of the world as the animal. Another example is provided by the work of McNamara et al. (2012) on state-dependent valuation.

With these examples, we are trying to illustrate that the macroscopic world of decisions is more complex than traditional models of decision theory assume. P\&B (sect. 5) write: "If we cannot assume an objective reality and an omniscient cognitive agent, then perhaps the perspective-driven probabilistic evaluation in quantum theory is the best practical rational scheme. In other words, quantum inference is optimal, for when it is impossible to assign probabilities to all relevant possibilities and combinations concurrently." We are not convinced that understanding the macroscopic world of decisions requires a step as dramatic as that of abandoning objective reality in the way that the quantum world does.

\section{Limitations of the Dirac formalism as a descriptive framework for cognition}

\section{doi:10.1017/S0140525X12003007}

\author{
Artem Kaznatcheev ${ }^{\mathrm{a}, \mathrm{b}}$ and Thomas R. Shultz ${ }^{\mathrm{a}, \mathrm{b}}$ \\ ${ }^{a}$ School of Computer Science; ${ }^{\mathrm{b}}$ Department of Psychology, Mc Gill University, \\ Montreal, QC H3A 1B1, Canada. \\ artem.kaznatchee@mail.mcgill.ca \\ thomas.shultz@mcgill.ca \\ http://www.cs.mcgill.ca/ akazna/ \\ http://www.tomshultz.net/
}

Abstract: We highlight methodological and theoretical limitations of the authors' Dirac formalism and suggest the von Neumann open systems approach as a resolution. The open systems framework is a generalization of classical probability and we hope it will allow cognitive scientists to extend quantum probability from perception, categorization, memory, decision making, and similarity judgments to phenomena in learning and development.

Pothos \& Busemeyer (P\&B) present the Dirac formalism of quantum probability (DQP) as a potential direction for cognitive modeling. It is important to stress that the quantum probability (QP) approach is a modeling framework. It does not provide guidance in designing experiments or generating testable predictions. It is not a theory. $\mathrm{P} \& \mathrm{~B}$ do not show how the framework could be used to build predictive theories: all the examples listed are post hoc descriptive models. Therefore, the target article should be judged on its merits as a descriptive framework.

A framework has to allow experimenters to average behavior over (or within) participants. Unfortunately, the pure states of $\mathrm{P} \& \mathrm{~B}$ 's Dirac formalism cannot be directly averaged. Experimental psychologists have addressed this methodological constraint of DQP by ad hoc fixes (Bruza et al. 2009; Franco 2009). To avoid such fixes or creating statistics outside the framework, one has to introduce mixed states (see sect. 2.4 of Nielsen \& Chuang [2000]), which physicists use to reason about open quantum systems.

A theoretical expressiveness constraint is that the time evolution in DQP is inherently periodic. After a recurrence time, the evolution will be arbitrary close to (experimentally indistinguishable from) the identity, as a consequence of the Poincaré recurrence theorem for closed quantum systems. In terms of decision making, this means that given enough deliberation time, a participant will always return to a mental state indistinguishable from the one before deliberation. As the QP framework has no natural time scales, this issue can be swept under the rug by hoping that the recurrence time is astronomical compared with typical deliberation. To overcome this limitation in a principled manner, we must look beyond the Dirac formalism and allow irreversible evolutions.

In quantum information theory, irreversible evolutions are introduced via quantum channels (see sect. 8.2 of Nielsen \& Chuang [2000]). Like the mixed states, and unlike P\&B's pure states and unitary evolution, quantum channels can be averaged; a methodological asset. The combination of quantum channels and mixed states is often described as the "von Neumann formalism" (NQP; or "Lindbland form" if we think in terms of master equations) and is the standard approach for dealing with open quantum systems. In physics, it is possible to alternate between NQP and DQP by explicitly modeling the environment to close the system. However, this is an unreasonable constraint for modeling an open system such as the mind. When modeling the decision "Is Linda a bank teller or feminist?" psychologists do not close the system by explicitly considering all the other concurrent thoughts (e.g., "What will I have for dinner?").

Modellers must choose between NQP and DQP. The preceding methodological and theoretical considerations compel us to select NQP. Because a Markov chain is a type of quantum channel, this choice makes the framework a generalization of classical probability (CP; see Kaznatcheev [submitted] for a complete treatment) and renders section 4.3 of the target article moot. We cannot use 
global properties of the transition operator (such as the law of totalprobability versus double-stochasticity) to empirically decide between $\mathrm{CP}$ and NQP, because any transition matrix consistent with CP will also be consistent with NQP.

If we take NQP as a generalization of CP, then we are faced with the difficulty of justifying the increase in complexity. Physicists are able to justify the difficulty of quantum mechanics over classical local hidden variable theories through experimental violations of Bell's inequality. Physical tests of Bell's inequality rely on being able to separate entangled subsystems (local property of the transition operator) far enough and perform measurements fast enough that the subsystems cannot communicate without violations of special relativity. Although Aerts and Sozzo (2011b) are able to produce joint probability functions that violate Bell's inequality in psychological settings, they cannot draw the strong conclusions of physicists because their experiments cannot create a space-like separation between the subsystems (in their case the systems are concepts within a single mind). In this psychological setting, local and global hidden variable theories cannot be distinguished, and a classical explanation cannot be ruled out. Without an operationalist test (such as Bell's) or reductionist grounding in the physics of quantum systems (which seems unlikely [Litt et al. 2006]), we are left with only practical considerations as criteria to decide between the CP and NQP frameworks.

Because QP is notorious for being unintuitive and difficult to understand, great care is required when trying to reconcile QP with classical intuition. For example, non-orthogonal states are impossible to perfectly distinguish (Chefles 2000). P\&B make this error in the classical reasoning of their model in section 3.1:

[I]n situations such as this, the more probable possible outcome is evaluated first...Therefore, the conjunctive statement involves first projecting onto the feminist basis vector, and subsequently projecting on the [non-orthogonal] bank teller one. (emphasis ours)

Unfortunately, the subject cannot determine the more overlapped state by any process inside QP. P\&B have to step outside their framework and provide an ad hoc fix of the sort they dismiss in CP.

From a psychological perspective, it is encouraging that the QP framework addresses issues in perception, categorization, memory, decision making, and similarity judgments. However, it lacks tools to cover learning and development. In contrast, Bayesian CP is capable of learning and inference (Griffiths et al. 2008), and has made inroads into modeling development (Shultz 2007). Comparable extensions of the QP framework would be welcomed. If QP cannot be extended to learning and development, then it is unlikely to replace the popular Bayesian CP approach.

In conclusion, we agree that a QP approach should be pursued in psychology, but we suggest viewing it from the open systems perspective of NQP. DQP is restricted to closed systems, but the mind is an open system, and, therefore, NQP yields a better modeling framework. However, it is unclear how to experimentally rule out ad hoc CP interpretations without physically separating psychological subsystems. P\&B's survey of QP's significant progress in covering a range of psychological phenomena is promising, and we look forward to future coverage of learning and development.

\section{Disentangling the order effect from the context effect: Analogies, homologies, and quantum probability}

\section{doi:10.1017/S0140525X12003159}

Elias L. Khalil

Department of Economics, Monash University, Clayton, Victoria 3800, Australia.

elias.khalil@monash.edu

http://eliaskhalil.com
Abstract: Although the quantum probability $(\mathrm{QP})$ can be useful to model the context effect, it is not relevant to the order effect, conjunction fallacy, and other related biases. Although the issue of potentiality, which is the intuition behind QP, is involved in the context effect, it is not involved in the other biases.

Why do we need quantum probability (QP) in place of classical probability (CP)? According to Pothos \& Busemeyer (P\&B), we need the QP technique to model "incompatible" questions, that is, when the states of the world are "potential" states that would collapse into concrete ones in light of interaction with other variables. This is similar to the famous Schrödinger's cat: The state of the cat is both dead and alive in the potential state, which becomes concrete only with further interaction. But if the questions are "compatible," the CP technique would suffice.

P\&B apply QP to the "context effect" as illustrated in the questions about happiness and employment. These questions are incompatible because the evaluation of one interferes with the evaluation of the other.

P\&B, in turn, apply QP to the "order effect" and other biases such as the conjunction fallacy, similarity judgment, and Wason selection task. In these biases, incompatibility again arises because a particular appearance of a question influences the answer.

But is a particular appearance - as in the "order effect" - the same as the "context effect"? The order effect and other biases definitely share similarities with the context effect-which seem to justify the use of the same technique, namely, the QP technique in their case.

However, there is problem: What if the similarities between the appearance effect, such as the order effect, and the context effect are analogies rather than homologies, to use the familiar biological distinction? If they are analogies, the use of the same technique (whether CP or QP) for the analysis of both effects would amount to an "identificational slip" (Khalil 2000). One would commit an identificational slip if one mistakes an analogy for a homologysuch as identifying the forearms of dolphins as "fins" or the forearms of bats as "wings" (Khalil 2000). To recall, the forearms of dolphins and bats are homologous because of common mammalian origin. In contrast, the forearms of dolphins and fish, or the forearms of bats and birds, are analogous because of common functions. One would commit an identification slip if one used the fin or the wing technique to describe how, respectively, dolphins swim or how bats fly. Therefore, do $\mathrm{P} \& \mathrm{~B}$ commit an identificational slip when they use the QP technique to describe the order effect (and other biases)?

First, what is the initial intuition behind QP? It is designed to model potentiality in nature, with implications with regard to the specification of meaning of what is observed by the sense, including the meaning of observed actions in relation to goals. The latter has ramifications for the understanding of internal motivation, self-esteem, and entrepreneurship (see Khalil 1997a; 1997b; 2010). Such potentiality is a "context," which becomes concrete with interaction.

Do the biases marshaled by $\mathrm{P} \& \mathrm{~B}$ as candidates for QP involve potentiality; a potentiality found in the context effect? With respect to the order effect, do we have a potentiality issue?

When subjects are presented with $\mathrm{A}$ and $\mathrm{B}$-for example, "Is Clinton honest?" and "Is Gore honest?" - they give different answers, depending on the order of A and B. The questions A and $\mathrm{B}$ invoke symmetrical and independent information. Information with regard to each question can be evaluated without the appeal to the other. But this is not the case with the employment and happiness questions. If you ask if one is happy, the evaluation must depend on another question, such as employment, because one must be happy about something. But if you ask "Is Clinton honest?" the evaluation need not depend on the honesty of Gore. Insofar as the Gore question exercises influence on how to assess Clinton (i.e., the order effect), it must have acted as a fast and frugal metric. Therefore, A and B cannot be "incompatible" questions: The presence of A does not act as a context for B. There is no potentiality that becomes concrete once the other piece is present. 
Commentary/Pothos \& Busemeyer: Can quantum probability provide a new direction for cognitive modeling?

It is simply that each metric has its own memories or heuristics. $\mathrm{P} \& \mathrm{~B}$ dismiss such an explanation, but it can be related to Herbert Simon's "bounded rationality." For Simon, the person has limited cognitive resources, and hence has to use rules of thumb to make decisions. Such rules of thumb are fast ways to look at a problem when the stakes are not high. That is, such rules of thumb are actually efficient, which explains why standard economists welcome Simon's notion of bounded rationality. To wit, if the stakes are high, people usually review and replace the existing rule of thumb (Baron 2008, Ch. 6). People are even ready to correct their errors of logical reasoning, as in the Wason selection task or the famous Linda in the conjunction fallacy, once they are given extra time for reflection and cognition.

Such correction would not be possible if the order effect were similar to the context effect in a substantial (homologous) sense. But what is "context"? It is the outcome of the neural principle when it specifies the background, which allows the comprehension of the foreground data; similar to how the brain selects the background in Rubin's vase. The principle that specifies the background cannot be "corrected" by data because it is not a datum. The background acts as a context that affords "meaning" or, as discussed earlier, a "potentiality" of what the observed thing can become.

An example of the context effect is the "gain frame" versus the "loss frame" in the Asian disease experiment: Two medical remedies are presented to deal with an Asian disease: one remedy has a certain outcome in terms of saved lives or prevention from death, whereas the other has uncertain outcomes. The uncertain remedy has an expected value equal to the certain remedy. Subjects usually chose the certain remedy if the results were couched as "saving lives" rather than "preventing death," that is, using the "gain frame," but subjects usually chose the uncertain remedy if the results were couched in the other way, that is, using the "loss frame."

LeBoeuf and Shafir (2003) showed that subjects, once exposed to a context, would not generally "correct" the choice if given more time to reflect, even if such subjects had scored high on the "need for cognition" test, that is, enjoyed cognitive pursuits (Cacioppo \& Petty 1982). This confirms that context is not a datum to be corrected. The context is rather a background that provides meaning to the foreground, content. The context and content are asymmetrical, that is, stand dependently on each other. This is contrary to the Gore/Clinton test in which A and B are symmetrical, that is, stand independently of each other. Therefore, the context effect is radically different from the order effect.

Therefore, $\mathrm{P} \& \mathrm{~B}$ commit an identificational slip: they confuse analogies with homologies. The same technique (whether CP or QP) cannot apply to the context effect, on the one hand, and to the order effect and its associated biases, on the other.

\section{The cognitive economy: The probabilistic turn in psychology and human cognition}

\section{doi:10.1017/S0140525X12003019}

\section{Petko Kusev ${ }^{\mathrm{a}, \mathrm{b}}$ and Paul van Schaik ${ }^{\mathrm{c}}$ \\ ${ }^{a}$ Department of Psychology, Kingston University London, London KT1 2EE, United Kingdom; ' ${ }^{\mathrm{b}}$ City University, London EC1V OHB, United Kingdom; 'Department of Psychology, Teesside University, Middlesbrough TS1 3BA, United Kingdom. \\ p.kusev@kingston.ac.uk \\ P.Van-Schaik@tees.ac.uk \\ http://kusev.co.uk \\ http://sss-studnet.tees.ac.uk/psychology/staff/Paul_vs/index.htm}

\footnotetext{
Abstract: According to the foundations of economic theory, agents have stable and coherent "global" preferences that guide their choices among alternatives. However, people are constrained by information-processing and memory limitations and hence have a propensity to avoid cognitive load. We propose that this in turn will encourage them to respond to "local" preferences and goals influenced by context and memory representations.
}

One of the most significant current discussions in economics and psychology is about the (lack of) link between normative (what we should do, based on probability and logic) and descriptive theories (what we do) of decision making. An obvious challenge for and advantage of good theory in decision making is that it is general, being able to account for both normative (rational) and descriptive psychological mechanisms and assumptions (Kusev et al. 2009). According to the foundation of economic theory, people have stable and coherent "global" preferences that guide their choices among alternatives varying in risk and reward. In all their variations and formulations, normative utility theory (von Neumann \& Morgenstern 1947), and descriptive prospect theory (Kahneman \& Tversky 1979; Tversky \& Kahneman 1992) share this assumption (Kusev et al. 2009). However, a consistent claim from behavioral decision researchers is that, contrary to the assumptions of classical economics, preferences are not stable and inherent in individuals but are "locally" constructed "on the fly" and are strongly influenced by context and the available choice options (e.g., Kusev et al. 2009; 2012a; 2012b; Slovic 1995). For example, the preference reversal phenomenon (Lichtenstein \& Slovic 1971; 1973) suggests that no stable pattern of preference underlies even basic choices; in other words, consistent trade-offs between lotteries with different probabilities and values are not made. Accordingly, we shall present some evidence for violations of the classical probability framework, inspired by existing research in judgment and decision making, and also review plausible sources for understanding human decision making: specifically simplicity in human information processing, based on local decision making goals and strategies.

Theorists in cognitive science achieve general theoretical propositions, based on the following assumptions: (1) the cognitive systems solve problems, optimally, given environmental and processing constrains (Anderson 1990; 1991); therefore, the objective is to understand the structure of the problem from the point of view of cognitive systems and (2) cognitive goals determine choice behavior: when a general cognitive goal is intractable, a more specific cognitive goal, relevant to achieving the general goal, may be tractable (Oaksford \& Chater 2007; 2009). For example, all local goals are assumed to be relevant to more general goals, such as maximizing expected utility. The observation that the local goals may be optimized as surrogates for the larger aims of the cognitive system raises another important question about the use of rational models of human cognition. Specifically, Oaksford and Chater (2007; 2009) propose that optimality is not the same as rationality. The fact that a model involves optimization does not necessarily imply a rational model; rationality requires that local goals are (1) relevant to general goals and (2) reasonable. Here we make a very simple assumption about how and whether the cognitive system optimizes. We assume that the cognitive system simplifies and adopts "local" goals, and that these goals will be influenced by contextual and memory representations. Accordingly, we argue, strengthening such an account could provide a challenge to classical probability approach.

Whereas some phenomena in judgment and decision making systematically violate basic probabilities rules - classic examples include the conjunction fallacy (Tversky \& Kahneman 1983), the disjunction effect (Tversky \& Shafir 1992), the subadditivity principle (e.g., Tversky \& Koehler 1994), and the preference reversal phenomenon (Lichtenstein \& Slovic 1971; 1973; Slovic 1995) - the simplicity framework suggested in this commentary argues that people are constrained by information-processing and memory limitations, and hence have a propensity to avoid cognitive load. Research in judgment and decision making demonstrates that by focusing on local goals (e.g., the representativeness heuristic) people may violate principles of classical probability theory (e.g., fallacies in which specific conditions are assumed to be more probable than a single general one). For example, the independence assumption states that the occurrence of one event makes it neither more nor less probable that the other occurs; examples of violation include the conjunction fallacy (Tversky \& Kahneman 1983), the disjunction effect 
(Tversky \& Shafir 1992), and the familiarity bias (e.g., Fox \& Levav 2000; Tversky \& Koehler 1994). One plausible account for these effects, as an alternative to classical logic, classical probability, and the classical information-processing paradigm, is quantum probability theory (Busemeyer \& Wang 2007; Busemeyer et al. 2006; 2011; target article, sects. 1 and 2). These authors argue that the "classical" view forces highly restrictive assumptions on the representation of the complex cognitive system. In particular, they suggest that (1) the brain is a complex information-processing system with a large number of unobservable states, (2) the brain is highly sensitive to context and, finally, (3) the measurements that we obtain from the brain are noisy and subject to uncertainty. Pothos \& Busemeyer $(\mathrm{P} \& \mathrm{~B})$ show that quantum probability theory allows the modeling of decision-making phenomena (e.g., the conjunction fallacy and violations of the sure-thing principle), going beyond classical probability theory. This is because quantum probability theory can account for context- and order-dependence of human behavior.

We conclude that the cognitive system is likely to respond to "local" goals (that might be tractable) influenced by memory representations and context that may be indicative of probability and frequency judgments. Therefore, in contrast to classical probability theory, quantum probability theory has the potential to account for context- and order-dependent behavior that is indicative of human propensity to adopt local goals. Moreover, there is mounting evidence for this type of behavior and simple mechanisms ruling human behavior (Kusev et al. 2011). Therefore, quantum probability has the potential to account for cognitive economy in many domains of human cognition.

\section{Quantum models of cognition as Orwellian newspeak}

\section{doi:10.1017/S0140525X12003020}

\section{Michael D. Lee ${ }^{\mathrm{a}}$ and Wolf Vanpaemel ${ }^{\mathrm{b}}$ \\ ${ }^{a}$ Department of Cognitive Sciences, University of California Irvine, Irvine CA 92697-5100; ${ }^{\mathrm{b}}$ Faculty of Psychology and Educational Sciences, University of Leuven, 3000 Leuven, Belgium. \\ mdlee@uci.edu \\ wolf.vanpaemel@ppw.kuleuven.be http://faculty.sites.uci.edu/mdlee/publications/ \\ http://ppw.kuleuven.be/okp/people/wolf_vanpaemel/}

Abstract: Faced with probabilistic relationships between causes and effects, quantum theory assumes that deterministic causes do not exist, and that only incomplete probabilistic expressions of knowledge are possible. As in its application to physics, this fundamental epistemological stance severely limits the ability of quantum theory to provide insight and understanding in human cognition.

Some physicists have serious objections to quantum theory. Their dissent does not relate to ontology - there is broad acceptance that quantum theory has led to surprising and accurate predictions about the physical world, with useful applied results-but, rather, to epistemology. As Pothos \& Busemeyer (P\&B) make clear in their target article, they are not making ontological claims about the brain being quantum but are arguing for the epistemological promise of the quantum approach offering psychological understanding of "the fundamental why and how questions of cognitive process" (sect. 1.2). Therefore, the criticisms from physics are directly relevant.

The physicists' argument against the quantum approach to modeling is clearly explained by Jaynes (2003, pp. 327-28). If there is an effect $\mathrm{E}$ that does not occur unless some condition $\mathrm{C}$ is present, it seems natural to infer that the condition is a necessary causative agent for the effect. If, however, the condition does not always lead to the effect, it seems natural to infer that there must be some other causative factor $\mathrm{F}$, which has not yet been understood. This is the approach taken by classical probability theory. Asking the Gore question $\mathrm{C}$ causes an opinion $\mathrm{E}$ to be produced. If the opinion is different when the Clinton question $\mathrm{F}$ is asked first, then the conditional probability $\mathrm{p}(\mathrm{E} \mid \mathrm{C})$ must be extended to $\mathrm{p}(\mathrm{E} \mid \mathrm{C}, \mathrm{F})$ to take account of the relevant additional information in factor or context F. Quantum theory, as advocated by the target article, takes a fundamentally different approach. Faced with probabilistic relationships between causes and effects, quantum theory assumes that deterministic causes do not exist, and that only incomplete probabilistic expressions of knowledge are possible. Rather than trying to understand the exact nature of the influence of the relevant factor F, quantum theory simply assumes this level of understanding is beyond its scope.

We think that it is reasonable to have reservations when dealing with something as complicated as human cognition, about whether it is practically feasible to isolate all the relevant factors and fully understand their interactions, but that is not the issue here. In sharp contrast to classical probability, adopting the quantum approach makes it nonsensical even to aim for this understanding. As Jaynes (2003, p. 328) puts it: "The mathematical formalism of present quantum theory, like Orwellian newspeak, does not even provide a vocabulary with which one could ask such a question." It is the difference between, in practice, not having the understanding of physics needed to control deterministically the outcome of a coin flip, versus assuming, in principle, that such a level of understanding can never be reached (i.e., that even with sophisticated instruments for applying forces and measuring initial conditions, the outcome of a physical coin flip is inherently probabilistic). The deterministic effects of order, context, and the other factors highlighted in the target article on human cognition might be hard to understand, but adopting the quantum approach requires us to stop trying.

The target article seems to acknowledge this hamstringing, when it argues that the basic quantum property of superposition is "an intuitive way to characterize the fuzziness (the conflict, ambiguity, and ambivalence) of everyday thought." (sect. 1.1) But should characterizing indefiniteness be the objective when building models of cognition? Surely the goal of modeling cognition is to sharpen our understanding, and remove the fuzziness, rather than to replicate it in the modeling machinery. The fact that people cannot unpack the relevant contexts or other factors that mediate their cognitive processes does not mean that our models must be similarly unable to do so. The same basic issue plays out in discussion of entanglement, which makes it impossible to construct a complete joint distribution between two variables, and therefore impossible to model how they interact with each other. Instead, the quantum approach implants indefiniteness about how cognition works directly into the modeling framework, and there is neither the possibility nor need to work hard to build better models that remove the confusion.

The target article argues that the acid test is "whether there are situations where the distinctive features of QP theory provide a more accurate and elegant explanation for empirical data." (sect. 1.2) Viewing quantum theory as newspeak implies that it cannot, in principle, lead to complete explanations. We think the worked examples in the target article bear this out. There are striking disconnects between the descriptions of the formal workings of the models, and their psychological interpretations. The former seem devoid of psychological content and the latter seem vague and verbal. For example, the projection of lines onto subspaces that models the conjunction fallacy, with its mathematical precision, is "explained" as "a kind of abstraction process, so that the projection on to the feminist subspace loses some of the details about Linda." (sect. 3.1)

Sloman's blurb for the Busemeyer and Bruza (2012) book on quantum models of cognition and decision says "Mathematical models of cognition so often seem like mere formal exercises. Quantum theory is a rare exception. Without sacrificing formal rigor, it captures deep insights about the workings of the mind with elegant simplicity." We think that is exactly the wrong way 
around. Quantum models of cognition offer formal exercises that might produce impressive fits to data but, by their founding assumptions, cannot offer some of the most basic insights into the causes, effects, and relevant factors that underlie the workings of human cognition.

Jaynes (1993, p. 269) puts the physicists' epistemological dissent bluntly, saying "I am convinced, as were Einstein and Schrödinger, that the major obstacle that has prevented any real progress in our understanding of Nature since 1927, is the Copenhagen Interpretation of Quantum Theory. This theory is now 65 years old, it has long since ceased to be productive, and it is time for its retirement." It would be unfortunate if a theory ready for retirement in its professional field of physics were to enjoy a second hobbyist career in psychology.

\section{Grounding quantum probability in psychological mechanism}

\section{doi:10.1017/S0140525X12003147}

\section{Bradley C. Love \\ University College London, Cognitive, Perceptual and Brain Sciences, London WC1H OAP, United Kingdom. \\ b.love@ucl.ac.uk \\ bradlove.org}

Abstract: Pothos \& Busemeyer (P\&B) provide a compelling case that quantum probability $(\mathrm{QP})$ theory is a better match to human judgment than is classical probability $(\mathrm{CP})$ theory. However, any theory (QP, CP, or other) phrased solely at the computational level runs the risk of being underconstrained. One suggestion is to ground QP accounts in mechanism, to leverage a wide range of process-level data.

Pothos \& Busemeyer (P\&B) make clear that quantum probability (QP) theory offers a rich array of theoretical constructs, such as superposition, entanglement, incompatibility, and interference, which can help explain human judgment. The authors illustrate how these concepts, which are strongly contrasted with the basic tenets of classical probability (CP) theory, can be used to accommodate aspects of human choice that deviate from normative CP accounts. For example, the conjunction fallacy is explained in terms of incompatible questions requiring sequential evaluation, which induces an interference effect.

Although new frameworks can provide novel insights, one worry is that QP will recapitulate some of the shortcomings of rational CP approaches by sticking to a computational-level analysis. To the authors' credit, they acknowledge how notions of optimality in $\mathrm{CP}$ approaches can be impoverished and not match the goals of the decision maker. However, these criticisms largely serve to question CP's status as the preferred normative account rather than question the wisdom of eschewing process-level considerations in favor of a computational-level analysis.

In a recent article with Jones (Jones \& Love 2011), we, too, critiqued rational (Bayesian) CP approaches to explaining human cognition, but our critique was broader in scope. Although many of our points are particular to the rational Bayesian program (which we refer to as "Bayesian Fundamentalism"), some of the central critiques apply equally well to any approach largely formulated at the computational level. The basic issue is that such accounts wall off a tremendous amount of related data and theory in the cognitive sciences, including work in attention, executive control, embodiment, and cognitive neuroscience, as well as any study using response time measures. It seems unlikely that a complete theory of cognition or decision making can be formulated when neglecting these insights and important constraints.

The suggestion offered in Jones and Love (2011), which we referred to as "Bayesian Enlightenment," is to integrate probability and mechanistic approaches. In the context of QP, one could imagine construing operations, such as projections to subspaces, as psychological operations that unfold in time, may have brain correlates, be limited in capacity, and change over development. Such an approach would retain the distinctive characteristics of QP while linking to existing theory and data.

Grounding QP in mechanism may offer a number of other advantages, such as better motivating the assumptions (that are psychological in nature) that make QP successful. Many of the effects considered in the target article require assumptions on the order in which statements are considered and the role context plays. These topics may be addressed in a principled manner when situated within a mechanism that aims to explain shifts in focus or attention. Such mechanistic models would also make clear what role QP plays in accounting for the results, as opposed to the ancillary assumptions.

The authors note that one key challenge is to anticipate new findings rather than simply accommodate existing data. Grounding QP ideas in mechanism may facilitate making a priori predictions. Once the move to mechanism is made, second generation questions can be asked, such as which QP model best accounts for human judgment. My guess is that moving away from evaluating general frameworks to testing specific proposals will hasten progress. As the authors note, it is very difficult to invalidate an entire framework, as ancillary assumptions can always be made (e.g., CP models can be modified to account for the main findings in the target article). In contrast, particular models can be evaluated using model selection procedures.

My prediction is that moving toward evaluating particular models grounded in mechanism will lead to a rapprochement between QP and CP approaches. For a view that allows for superposition, many aspects of the QP are very rigid. For example, according to the approach advocated by the authors, statements are either compatible or incompatible. One possibility is that successful models will be more fluid and include a mixture of states, which is a notion from CP. Given the complexities of human cognition and decision making, it would be surprising if one unadulterated formalism carried the day. Although physics undergraduates may complain about how confusing QP is, human cognition will likely prove more vexing.

\section{Cognition in Hilbert space}

\section{doi:10.1017/S0140525X1200283X}

\section{Bruce James MacLennan \\ Department of Electrical Engineering and Computer Science, University of Tennessee, Knoxville, Knoxville, TN 37996. \\ maclennan@utk.edu \\ http://web.eecs.utk.edu/ mclennan/}

Abstract: Use of quantum probability as a top-down model of cognition will be enhanced by consideration of the underlying complex-valued wave function, which allows a better account of interference effects and of the structure of learned and ad hoc question operators. Furthermore, the treatment of incompatible questions can be made more quantitative by analyzing them as non-commutative operators.

Pothos \& Busemeyer (P\&B) argue for the application of quantum probability $(\mathrm{QP})$ theory to cognitive modeling in a function-first or top-down approach that begins with the postulation of vectors in a low-dimensional space (sect. 2.1), but consideration of the highdimensional complex-valued wave function underlying the state vector will expand the value of QP in cognitive science. To this end, we should import two premises from quantum mechanics. The first is that the fundamental reality is the wave function. In cognitive science, this corresponds to postulating spatially distributed patterns of neural activity as the elements of the cognitive state space. Therefore, the basis vectors used in QP are basis functions for an infinite (or very high) dimensional Hilbert space. The 
second premise is that the wave function is complex valued and that wave functions combine with complex coefficients, which is the main reason for interference and other non-classical phenomena. The authors acknowledge this (sects. 2.3, 3.3, Appendix), but they do not make explicit use of complex numbers in the target article.

There are several possible analogs in neurophysiology of the complex-valued wave function, but perhaps the most obvious is the distribution of neural activity across a region of cortex; even a square millimeter of which can have hundreds of thousands of neurons. The dynamics are defined by a time-varying Hamiltonian, with each eigenstate being a spatial distribution of neurons firing at a particular rate. The most direct representations of the magnitude and phase (or argument) of a complex quantity are the rate and relative phase of neural impulses.

The target article specifies that a decision corresponds to measurement of a quantum state, which projects the cognitive state into a corresponding eigenspace, but it is informative to consider possible mechanisms. For example, the need to act definitely (such as coming to a conclusion to answer a question) can lead to mutually competitive mechanisms, such as among the minicolumns in a macrocolumn, which create dynamic attractors corresponding to measurement eigenspaces. Approach to the attractor amplifies certain patterns of activity at the expense of others. Orthogonal projectors filter the neural activity and win the competition with a probability proportional to the squared amplitude of their inner products with the wave function. (In the case in which impulse phases encode complex phases, matching occurs when the phases are delayed in such a way that the impulses reinforce.) The winner may positively reinforce its matched signal components while the loser negatively reinforces its matched components. Regardless of mechanism, during collapse, the energy of the observed eigenstate of the question (measurement) operator captures the energy of the orthogonal eigenstates (this is the effect of renormalization). The projection switches a jumble of frequencies and phases into a smaller, more coherent collection, corresponding to the outcome (observed) eigenspace. This competition also explains the prioritization of more likely outcomes (sect. 3.1).

The target article (sect. 2.1) suggests that a QP model of cognition begins by postulating basis vectors and qualitative angles between alternative question bases (significantly, only real rotations are discussed). As a consequence, a QP model is treated as a low-dimensional vector space. This is a reasonable, top-down strategy for defining a QP cognitive model, but it can be misleading. There is no reason to suppose that particular question bases are inherent in a cognitive Hilbert space. There may be a small number of "hard-wired" questions, such as fight-or-flight, but the vast majority is learned. Certainly this is the case for questions corresponding to lexical categories such as (un-)happy and (un-)employed.

Investigation of the dynamics of cognitive wave function collapse would illuminate the mechanisms of decision making, but also the processes by which observables are organized. This would allow modeling of changes in the question bases, either temporary through context effects, or longer lasting through learning. Furthermore, many question bases are ad hoc, as when we ask, "Do you admire Telemachus in the Odyssey?" How such ad hoc projectors are organized requires looking beneath a priori basis vectors to the underlying neural wave functions and the processes shaping them.

Certainly one of the most interesting consequences of applying to QP to cognition is the analysis of incompatible questions. The approach described in the target article (sect. 2.2) begins by postulating that incompatible questions correspond to alternative bases for a vector space. The qualitative angle between the question bases is estimated by a priori analysis of whether the questions interfere with each other.

In quantum mechanics, however, the uncertainty principle is a consequence of non-commuting measurement operators, and the degree of non-commutativity can be quantified. Two measurement operators $P$ and $Q$ commute if $P Q=Q P$, that is, if the operator $P Q-Q P$ is identically 0 . If they fail to commute, then $P Q-Q P$ measures the degree of non-commutativity, which is expressed in quantum mechanics by the commutator $[P, Q]=P Q-Q P$. It is relatively easy to show that this implies an uncertainty relation: $\Delta P \Delta Q \geq|\langle[P, Q]\rangle|$. That is, the product of the uncertainties on a state is bounded below by the absolute mean value of the commutator on the state. Suppose $H$ is a measurement that returns 1 for $\mid$ happy $\rangle$ and 0 for |unhappy , and $E$ is a measurement that returns 1 for $\mid$ employed $\rangle$ and 0 for $\mid$ unemployed $\rangle$. If

$$
\begin{aligned}
& \mid \text { employed }\rangle=a \mid \text { happy }\rangle+b \mid \text { unhappy }\rangle \\
& \text { then the commutator is }[H, E]=a b\left(\begin{array}{cc}
0 & 1 \\
-1 & 0
\end{array}\right)
\end{aligned}
$$

and the magnitude of the commutator applied to an arbitrary state $|\psi\rangle$ is $\|[H, E]|\psi\rangle\rangle \|=|a b|$.

Might we design experiments to measure the commutators and so quantify incompatibility among questions? Certainly there are difficulties, such as making independent measurements of both $P Q$ and $Q P$ for a single subject, or accounting for intersubject variability in question operators. But making such measurements would put more quantitative teeth into QP as a cognitive model.

\section{Processes models, environmental analyses, and cognitive architectures: Quo vadis quantum probability theory?}

\author{
doi:10.1017/S0140525X12003032
}

\author{
Julian N. Marewski and Ulrich Hoffrage \\ University of Lausanne, Quartier UNIL-Dorigny, 1015 Lausanne, Switzerland. \\ Julian.Marewski@unil.ch \\ Ulrich.Hoffrage@unil.ch \\ http://www.hec.unil.ch/people/jmarewski \\ http://www.hec.unil.ch/people/uhoffrage
}

Abstract: A lot of research in cognition and decision making suffers from a lack of formalism. The quantum probability program could help to improve this situation, but we wonder whether it would provide even more added value if its presumed focus on outcome models were complemented by process models that are, ideally, informed by ecological analyses and integrated into cognitive architectures.

In the cognitive and decision sciences, much research suffers from a lack of formalism. This is particularly the case for qualitative accounts of behavior proposed, for instance, within the heuristics-and-biases framework (Kahneman et al. 1982), or within related dual process theories of cognition (Sloman 1996). We applaud Pothos \& Busemeyer's (P\&B's) attempt to promote a formal framework that contributes to remedying this shortcoming and that has a high potential for being innovative and useful. With that being said, we take issue with three aspects of the quantum probability $(\mathrm{QP})$ program.

First, we posit that outcome models should be complemented by process models. What level of description do P\&B envision for QP models? One of the central goals of many psychological theories is to describe cognitive processes. In contrast, behavioral economists and cognitive scientists working with, for example, Bayesian models (e.g., Griffiths et al. 2008) focus on predicting the outcomes of behavior, without necessarily aspiring to provide plausible accounts of the underlying processes (Berg \& Gigerenzer 2010). We worry that the QP program falls into this class of outcome-oriented (or as-if) models, banishing algorithmic-level accounts of memory, 
perceptual, motivational, and decisional processes into the behaviorist's black box. We challenge P\&B to demonstrate how the QP framework can contribute to developing process models such as those proposed by the adaptive toolbox program (Gigerenzer \& Selten 2001). The repertoire of fast-and-frugal heuristics developed in this program includes algorithmic rules that specify how the cognitive system searches for information, when it will stop searching, and how it will combine the acquired pieces of information in order to make a decision. Consider, for instance, the priority heuristic (Brandstätter et al. 2006). This simple lexicographic strategy for making risky choices between gambles is composed of three rules that operate on the probability and outcome vectors constituting the gambles. For example, the first rule prescribes in what order the information contained in these vectors is searched. The rules of these and other heuristics have been used to motivate process predictions about reaction times (Bröder \& Gaissmaier 2007), eye movements (Reisen et al. 2008), functional magnetic resonance imaging (fMRI) data (Volz et al. 2010), and the amount of information looked up on a computer screen (Johnson et al. 2008). Process models help to gain a deeper understanding of behavior, and their predictions - albeit not always free of scholarly disputes (e.g., Marewski et al. 2010; 2011)-can eventually differentiate among competing theories that make identical outcome predictions.

Second, we posit that the process models we ask for should be informed by an analysis of the structure of the environment - after all, it takes two to tango. As has been stressed by many cognitive modelers (Anderson \& Schooler 1991; Dougherty et al. 1999; Gigerenzer et al. 1991; Oaksford \& Chater 1998; Simon 1956), the human cognitive system is adapted to its ecology. To use Brunswik's (1964) metaphor, mind and environment are like spouses who have co-evolved, who mutually shaped each other, and who cannot and should not be separated. The heuristicsand-biases program does not consider the situated nature of cognitive processes; rather, typically its heuristics are hypothesized to be invoked independent of context and there is no ecological analysis specifying how performance depends on environmental factors (Gigerenzer 1996). P\&B use the heuristics that have been proposed in the heuristics-and-bias tradition to motivate the QP program, pointing out that these heuristics reveal the insufficiencies of the classical probability program. While the QP program addresses some of these insufficiencies, others might be remedied by more explicitly considering (1) how environmental structures have shaped cognitive processes, (2) how such structures can select cognitive processes, and (3) how these processes, once selected, perform differentially in environments with different statistical structures. For instance, the shape of the distribution of environmental variables influences the performance of the QuickEst and social-circle heuristics, two fastand-frugal heuristics for estimating quantities and frequencies, respectively (Hertwig et al. 1999; Pachur et al. 2013). More examples of how the functioning of cognitive processes depends on environmental structures and how an analysis of the environment can assist researchers to develop - what one might term ecological process models can be found in Ecological Rationality: Intelligence in the World (Todd et al. 2012) and in Simple Heuristics in a Social World (Hertwig et al. 2013).

Third, we posit that such ecological process models should be integrated into cognitive architectures. As P\&B correctly point out, any theory requires constraints. One way to constrain psychological theories consists of introducing axioms and positing that cognition adheres to these axioms. Another way to constrain theories requires formulating psychologically grounded guiding principles that reflect empirical observations about behavior (cf. Anderson \& Lebiere 2003). Consistent with the latter approach, we and others (Marewski \& Mehlhorn 2011; Marewski \& Schooler 2011; Nellen 2003; Schooler \& Hertwig 2005) have modeled a few of the fast-and-frugal heuristics - including their dependencies on environmental, mnemonic, and perceptual variables - with the ACT-R architecture (Anderson et al. 2004). This architecture integrates models of memory, perception, and other components of cognition into one unified theory. In doing so, ACT-R incorporates the findings of decades of empirical work into computer code and mathematical equations, which, in turn, helps to constrain new models of cognition - including heuristics when these are implemented in the architecture. We wonder to what extent the QP program's axiomatic approach provides added value for constraining ecological process models beyond what can be achieved by implementing them into psychologically plausible architectures. We hasten to add that we also wonder whether the QP program's axiomatic approach might aid, conversely, in further refining such architectures.

To conclude, we believe the QP program may do a lot of good. However, we ask ourselves whether it could do even better if (1) its presumed focus on outcome models were complemented by process models that (2) are, ideally, informed by ecological analyses and (3) integrated into cognitive architectures.

\section{The implicit possibility of dualism in quantum probabilistic cognitive modeling}

\author{
doi:10.1017/S0140525X12003044
}

\section{Donald Mender \\ Department of Psychiatry, Yale University, New Haven, CT 06511. donald.mender@yale.edu}

\begin{abstract}
Pothos \& Busemeyer $(\mathrm{P} \& \mathrm{~B})$ argue convincingly that quantum probability offers an improvement over classical Bayesian probability in modeling the empirical data of cognitive science. However, a weakness related to restrictions on the dimensionality of incompatible physical observables flows from the authors' "agnosticism" regarding quantum processes in neural substrates underlying cognition. Addressing this problem will require either future research findings validating quantum neurophysics or theoretical expansion of the uncertainty principle as a new, neurocognitively contextualized, "local" symmetry.
\end{abstract}

Pothos \& Busemeyer's (P\&B's) answer to the question posed by the title of their article, "Can quantum probability provide a new direction for cognitive modeling?" addresses almost all the relevant issues. In particular, from an empirical viewpoint, the authors offer substantial evidence that quantum probability, explicated through its most transparent mathematical representation in generalized phase space, fits a great mass of cognitive data more closely than does classical Bayesian probability. P\&B show through numerous examples that this superior fit applies with respect to quantum probability's superpositional characteristics, unitarily time-evolved interfererence effects, entangled composition, and mutual incompatibility of precision in measurements entailing ordered context for certain pairs of observables enlisted as operators.

However, the word "certain" in the last sentence points toward one residual hole in the authors' agenda. That defect is their professed "agnosticism" regarding possibilities for physical embodiment of quantum probability in the thinking brain. Such agnosticism opens up an anarchical lack of limitation in choosing those particular pairs of observables that must be treated as mutually incompatible in cognitive applications of quantum probability.

Physicists as a rule take incompatibility between operator pairs to be a concept whose applicability is restricted only to "certain" that is, canonically conjugate physical-coordinates. Examples of canonically conjugate coordinate pairs include energy yoked to time and momentum yoked to distance. Each such pair yields a quantitative product with the qualitative dimensionality of action applicable to Planck's constant at the foundation of quantum mechanics and to the path-integrated functionals of quantum field theory.

If $\mathrm{P} \& \mathrm{~B}$ were to follow the restrictive practices of physicists by limiting their choices of operator pairs to canonically conjugate 
Commentary/Pothos \& Busemeyer: Can quantum probability provide a new direction for cognitive modeling?

observables, then physical embodiment of mental cognition in corporeal brain processes would be easy to infer. However, the authors also propose to consider for quantum probability theory qualitatively mentalistic observables, for example "happiness" or "feminism," entirely abstracted from the material brain, and non-corporeally applied to mind. Some such observables might conceivably be posited as mutually incompatible, non-commutatively order-sensitive sets of operators, even if they have been arbitrarily untethered from the physical anchor of canonical conjugation and the dimensionality of action, but that kind of rash detachment from the canonically conjugate constraints of physics could condemn cognitive science to a new form of Cartesian dualism. There would issue mentalistic forms of superposable probability amplitudes pertaining to properties and extended in "spaces" whose unitary "evolutions," mutual interference "effects," entangled "non-locality," and Fourier-dual "measurement" uncertainties lack any necessary connections to established physical observables and space times (or, alternatively, to their spin-networked foundations per loop quantum gravity) in which brains exist.

These caveats are especially germane if one stipulates that cognition, beyond the empirical question of its conformity to quantum probability, is formally quantum computational. The latter possibility remains an open biophysical question, insofar as classically engineered hardware can only imperfectly simulate but not fully support a quantum mode of abstract computation. As $\mathrm{P} \& \mathrm{~B}$ mention at the end of their article, nobody really knows yet whether the brain's biophysics are compatible with the coherence of putative quantum wetware processes. Some, such as Tegmark (2000), have championed principled thermodynamic objections to any such brain capabilities. Rebuttals by Hameroff and others (Hagan et al. 2002) to Tegmark's concerns about thermal decoherence have centered on two classes of argument. First, recent experimental evidence that quantum physics plays a role in photosynthesis (Engel et al. 2007) has been invoked against the idea that temperatures far colder than those found in living biological systems are needed to sustain quantum-coherent states. Second, proposals for thermal insulators that might protect physical quantum processes in the brain have been advanced. These include, for example, ordered water and pumped phonons, but to date none have been proven experimentally to operate in the relevant biological contexts.

Let us suppose that Tegmark's opponents turn out to be wrong, and quantum wetware substrates are nowhere to be found in neural tissue. If it transpires that the brain cannot sustain a quantum-biophysical "wetware" for cognition, then it will be hard to see how quantum-probabilistic "software" can offer a compelling perspective on cognitive phenomena within the framework of known metaphysical monisms. Thereafter those cognitive phenomena whose statistics conform more closely to abstract quantum probability than to classical Bayesian probability will be stuck between a rock and a hard place. Either quantum probability's disembodied elegance will have to be given up, or a new kind of dualism will be needed for cognitive science.

Making unified sense of that dualism will perhaps require some kind of trans-physical, post-Cartesian gauge, mimicking the generators of standard physical forces through coordination among "certain" local symmetries, but bridging quantitative intervals in "spacetimes" that, unlike the standard gauge fields of today's physics, are in a qualitative sense not purely physical. The new "gauge" would be required both to contact and to transcend corporeal physics while either obeying accepted physical laws or compensating against its own violations of those laws. A crucial underlying supposition in constructing such a psychophysical gauge might be the "local" character of canonically conjugate Fourier-duality as a kind of symmetry embedded within the "metrical" contexts of qualitatively neurocognitive "spaces" and "times." The specifics of that psychophysical gauge's design, which will have to confront head on Chalmers" "hard problem" of generally relating perceptual qualia and physical quantitation (Chalmers 1995), could well prove to be the most daunting neuroscientific challenge for any quantum probabilistic cognitive paradigm.

\section{What are the mechanics of quantum cognition?}

\section{doi:10.1017/S0140525X12003056}

\section{Daniel Joseph Navarro ${ }^{a}$ and lan Fuss ${ }^{b}$ \\ ${ }^{a}$ School of Psychology, University of Adelaide, SA 5005 Australia; ${ }^{\text {b School of }}$ Electrical Engineering, University of Adelaide, SA 5005 Australia. daniel.navarro@adelaide.edu.au ifuss@eleceng.adelaide.edu.au}

Abstract: Pothos \& Busemeyer (P\&B) argue that quantum probability (QP) provides a descriptive model of behavior and can also provide a rational analysis of a task. We discuss QP models using Marr's levels of analysis, arguing that they make most sense as algorithmic level theories. We also highlight the importance of having clear interpretations for basic mechanisms such as interference.

What kind of explanation does a cognitive model offer? A standard way of approaching this question is to use Marr's (1982) three levels of explanation. A "computational analysis" provides an abstract description of the problem that the learner must solve, along with a normative account of how that problem should be solved. Bayesian models of cognition are usually computational level explanations. An "algorithmic level" explanation describes a mechanistic process that would produce human-like behavior in some task. Most traditional information-processing models and many connectionist models lie at this level of explanation. Finally, "implementation level" explanations propose a low-level physical explanation of how the brain might perform the computations that are required. These are the kinds of models typically pursued in cognitive neuroscience.

Whereabouts in this classification scheme should we place the quantum probability (QP) framework? The implementation level is the simplest to consider. Pothos \& Busemeyer ( $\mathrm{P} \& \mathrm{~B})$ explicitly disavow any implementation level interpretation of these models; making a clear distinction between their work on the formal modeling of cognition using a quantum formalism and those researchers (e.g., Hameroff 1998) who argue that neural function should be modeled as a quantum physical system. We agree with this distinction.

Should QP models be treated as computational level analyses? Although P\&B make explicit comparisons to classical probability and to Bayesian models, we do not think it makes sense to treat QP models as computational level analyses. The critical characteristic of a computational analysis is to specify what problem the learner is solving, and to present a normative account of how that problem should be solved. Bayesian models work well as computational analyses because of the fact that classical probability provides good rules for probabilistic inference in everyday life. In discussing this issue, $\mathrm{P} \& \mathrm{~B}$ point to problems associated with statistical decision theory (e.g., that Dutch books are possible in some cases), or to well- known issues with the Kolmogorov axioms (e.g., sample spaces are hard to define in real world contexts). However, in our view their discussion misses the forest for the trees: showing that classical probability has limitations does not establish QP as a plausible alternative. There is a good reason why statistics is built on top of classical probability and not quantum probability: it is the right tool for the job of defining normative inferences in everyday data analysis. In contrast, although there are such things as "quantum $t$-tests" (e.g., Kumagai \& Hayashi 2011), they have yet to find a natural role within everyday statistical analysis. It is possible that such usage may emerge in time, 
but we think this is unlikely, simply because the situations to which such tools are applicable (e.g., data follow a quantum Gaussian distribution) do not arise very often when one is analyzing real data. Until statistical tools based on QP find a place in everyday data analysis, we remain unconvinced that QP makes sense as a normative account of everyday inference.

Regarding the algorithmic level, we think that $\mathrm{P} \& \mathrm{~B}$ are on more solid ground: there is some justification for thinking about QP models as mechanistic accounts. Consider the model used to account for Shafir and Tversky's (1992) data on the prisoner's dilemma. It relies on an interference effect to account for the fact that participants defect whenever the opponent's action is known but cooperate when it is unknown. This interference does not emerge as part of an optimal solution to the inference problem given to the decision maker, nor is it characterized at a neural level. It is clearly intended to refer to a psychological mechanism of some kind.

In view of this, a mechanistic view of $\mathrm{QP}$ seems to provide the right way forward, but at times it is difficult to understand what the mechanisms actually are. To take a simple example, why are some questions incompatible and others are compatible? $\mathrm{P} \& \mathrm{~B}$ suggest that "[a] heuristic guide of whether some questions should be considered compatible or not is whether clarifying one is expected to interfere with the evaluation of the other" (sect. 2.2). This seems sensible, but it begs the question. One is naturally led to ask why some psychological states interfere and others do not. This is difficult to answer because the QP formalism is silent on how its central constructs (e.g., interference) map onto psychological mechanisms. In our own work (Fuss \& Navarro, in press) we have explored this issue in regards to the dynamic equations that describe how quantum states change over time. Specifically, we have sought to describe how these equations could arise from mechanistic processes, but our solution is specific to a particular class of models and we do not claim to have solved the problem in general. In our view, understanding how formalisms map onto mechanisms is one of the biggest open questions within the QP framework.

In short, we think that the potential in QP lies in developing sensible, interpretable psychological mechanisms that can account for the otherwise puzzling inconsistencies in human decision making. It might be that human cognition cannot be described using the standard provided by classical probability theory, but turns out to be more consistent with QP theory. That doesn't make QP a good tool for rational analysis, but it would make it an interesting psychological mechanism, particularly if it is possible to provide clear and consistent interpretations for its central constructs. Should events unfold in this way, then statistics would continue to rely on classical probability for its theoretical foundation, but cognitive modelers could use quantum probability in many instances. There is nothing incompatible about these two states.

\section{A quantum of truth? Querying the alternative benchmark for human cognition}

\section{doi:10.1017/S0140525X12003068}

Ben R. Newell, Don van Ravenzwaaij, and Chris Donkin
School of Psychology, University of New South Wales, Sydney, 2052 NSW,
Australia.
ben.newell@unsw.edu.au
d.vanravenzwaaij@unsw.edu.au
c.donkin@unsw.edu.au
http://www2.psy.unsw.edu.au/Users/BNewell
http://www.donvanravenzwaaij.com
http://www2.psy.unsw.edu.au/users/cdonkin

Abstract: We focus on two issues: (1) an unusual, counterintuitive prediction that quantum probability $(\mathrm{QP})$ theory appears to make regarding multiple sequential judgments, and (2) the extent to which $\mathrm{QP}$ is an appropriate and comprehensive benchmark for assessing judgment. These issues highlight how QP theory can fall prey to the same problems of arbitrariness that Pothos \& Busemeyer (P\&B) discuss as plaguing other models.

1. Multiple sequential judgments. One of the basic tenets of quantum probability (QP) is that the order in which questions are asked of a person will affect how he or she feels about the answer. Pothos \& Busemeyer $(\mathrm{P} \& \mathrm{~B})$ illustrate this sequential nature of QP using the Clinton/Gore attitude assimilation effect reported by Moore (2002). The key result is that the percentage of participants endorsing Clinton as honest increases by $7 \%$ when Clinton is rated after Gore, but Gore's honesty endorsement decreases by $8 \%$ when he is asked about after Clinton. Thus the politicians become more similar (assimilate) when they are asked about second ( $3 \%$ difference in endorsement rates) than when asked about first ( $18 \%$ difference in endorsement rate). This point is illustrated in Figure 3 of $P \& B$, reprinted here as the top-left panel of Figure 1.

$\mathrm{P} \& \mathrm{~B}$ show that if the initial state vector is projected onto the Gore yes > basis vector first, followed by the |Clinton yes $>$ basis vector, Clinton will be judged as more honest than if the initial state vector is projected onto |Clinton yes $>$ directly. Thus, the authors explain how asking about the honesty of Gore first, will lead to a subsequently more positive judgment of Clinton's honesty.

An unusual prediction that follows is that as these projections continue, the state vector will gravitate toward the zero point. As an illustration, consider the effect of asking successive questions about the honesty of additional presidents. We assume that subsequent questions have representations as basis vectors in the outcome space. Just as the state vector from |Gore Yes> is projected onto |Clinton Yes $>$, we assume that subsequent questions cause the state vector to project onto the next appropriate basis vector. As shown in Figure 1, as each state vector projects onto the nearest point of the next basis vector, subsequent state vectors will get shorter (by definition).

Although we agree that asking about the honesty of a number of politicians might put one in a progressively more suspicious frame of mind, it seems unlikely that the believability of any president should necessarily decrease (reaching close to zero in as few as 10 questions) as more questions are asked. Imagine, for example, if the sixth president was Lincoln or Washington.

A possible solution to this problem is to assume that the state vector somehow resets or recalibrates itself, perhaps because of a decay of the effect of initial questions (i.e., forgetting). $P \& B$ argue that one of the benefits of QP is that it is based on axiomatic principles, thus avoiding problems of "arbitrariness" common in other explanatory frameworks (e.g., heuristics). Adding a "recalibration" step would appear to be a post-hoc fix outside of the main principles, and as such, something that $\mathrm{P} \& \mathrm{~B}$ are at pains to avoid. This example highlights why formal frameworks make such attractive theoretical tools: they make strong, testable predictions.

2. An appropriate benchmark? Two criteria have been prominent in the search for an appropriate benchmark for probability judgment: correspondence and coherence (eg., Hammond 1996). These terms, stemming from philosophy, invite different ways of assessing truth: via correspondence with observable facts, and via having a set of internally consistent (coherent) beliefs. Several commentators have argued that both criteria need to be considered for adequate assessment of judgments (e.g., Dunwoody 2009; Newell 2013).

$\mathrm{P} \& \mathrm{~B}$ argue strongly that coherence should be assessed against the axioms of QP not CP - hence allowing Linda to be more likely a feminist bank teller than just a bank teller - but what of correspondence? Consider the correspondence error that homicide is judged the more likely cause of death than suicide (e.g., Lichtenstein et al. 1978). Such a judgment is an error because it 

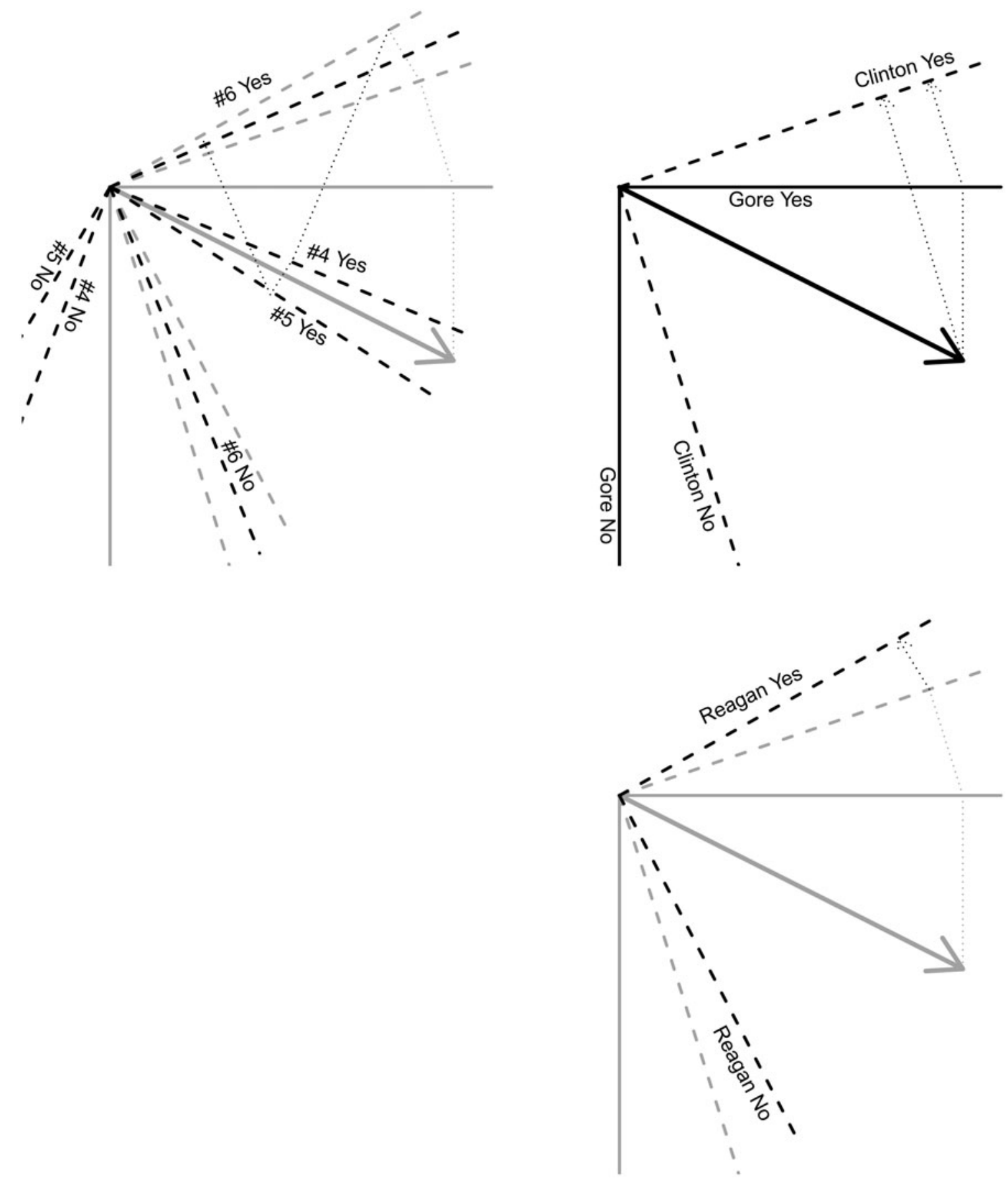

Figure 1 (Newell et al.). Multiple sequential judgments lead to a belief state that comes ever closer to zero. See text for details.

does not correspond with the fact that there are more suicides per capita than homicides. Such an "irrational" judgment emerges from the same cognitive system as the Linda judgment and, therefore, should, according to P\&B's thesis, be explicable in the QP framework. Our intuition is that QP theory would explain this effect by constructing bases corresponding to representations of death from suicide, death not from suicide, death from homicide, and death not from homicide (in much the same way as bases are constructed for happy and happy in P\&B's Fig. 1). It might be assumed that people's initial state vector, because of something akin to "availability," is closer to the homicide basis vector than the suicide vector. This would lead to a larger projection, and, therefore, a judgment of higher probability of homicide than suicide.
Assuming that it is possible to construct such a space, one may ask what predictions QP theory would make were we to ask the participants to sequentially judge the likelihood of both suicide and homicide. To generate such predictions, however, we must first know whether, for example, the two questions are compatible. We must also know whether the initial vector lies between the homicide and suicide basis vectors, or between the homicide and not suicide vectors, for example. Such decisions about the parameters of the model influence the qualitative pattern that QP theory will produce, for example, that compatibility will determine whether we expect the judgments to be invariant to the order of the questions. Similarly, the location of the initial state vector, for incompatible questions, will determine whether the second judgment increases or decreases relative to when it was 
judged first. Although not relevant to the current example, the principles of entanglement and superposition have similar effects on the qualitative pattern that QP theory predicts.

To call the decisions about such principles in QP theory "arbitrary" may be going too far $-\mathrm{P} \& \mathrm{~B}$ provide intuition for when we might expect some of these principles to hold (e.g., compatibility). However, we argue that an understanding of these unique aspects of QP theory, to the point that they are predictable, is a major issue that needs addressing before QP theory can vie to be the framework of choice.

\section{Quantum modeling of common sense}

\author{
doi:10.1017/S0140525X1200307X
}

\section{Hamid R. Noori and Rainer Spanagel \\ Institute of Psychopharmacology, Central Institute for Mental Health, Medical Faculty Mannheim, University of Heidelberg, J 5, 68159 Mannheim, Germany. hamid.noori@zi-mannheim.de \\ rainer.spanagel@zi-mannheim.de \\ http://www.zi-mannheim.de/psychopharmacology.html}

\begin{abstract}
Quantum theory is a powerful framework for probabilistic modeling of cognition. Strong empirical evidence suggests the contextand order-dependent representation of human judgment and decisionmaking processes, which falls beyond the scope of classical Bayesian probability theories. However, considering behavior as the output of underlying neurobiological processes, a fundamental question remains unanswered: Is cognition a probabilistic process at all?
\end{abstract}

Using quantum theory for understanding cognitive processes was mainly inspired by the Copenhagen interpretation of quantum mechanics in the 1920s. However, it took scientists almost a century to formalize cognitive models utilizing the unique features of quantum probability (Aerts \& Aerts 1995).

Based on the Kolmogorov probability axioms, classical theories rely on intuitive mathematical foundations that have substantiated their application for modeling psychological phenomena for a long time. Nevertheless, a large body of evidence on order and context dependent effects and the violation of the law of total probability suggest that human judgment might follow counterintuitive principles.

One of the major achievements of quantum theory was the conceptualization of the superposition principle. Measurements of a system, which is linearly composed by a set of independent states, assign it to a particular state and stop it from being in any other one. As a consequence, the act of observation influences the state of a phenomenon being observed, and indicates a general dependency on the order of observations. These are two significant aspects of cognitive processes that dramatically challenge the classical theories.

The lack of reliable mathematical formalisms within the classical frameworks to address such issues, the inclusion of the classical assessments as specific (trivial) quantum assessments, and the wider application range of quantum probability, even suggest the superiority of quantum theory for modeling cognition.

Pothos \& Busemeyer (P\&B) discuss this matter thoroughly and elegantly. Modeling non-commutative processes is a unique characteristic of quantum theory, which provides a noticeable distinction between compatible and incompatible questions for cognitive systems. From the psychological point of view, incompatibility between questions refers to the inability of a cognitive agent in formulating single thoughts for combinations of corresponding outcomes. Each question influences the human state of mind in a context-dependent manner and, therefore, affects the consideration of any subsequent question (induced order dependency). Bayesian models simply fail to represent the context- and order-dependent scenarios, whereas for non-dynamic compatible questions their predictions converge to the assessment of the quantum probability theories.

In addition to the more general nature of quantum theory in describing static cognitive processes, this formalism could also be viewed as an extension of the classical probabilities for dynamic processes. Whereas time evolution is in both theories represented as linear transformations, the dynamic quantum probabilities are nonlinear functions, which in general implicate possible violations of the law of total probability.

In the long run, the framework of quantum modeling of cognitive processes presents a generalization of the Bayesian theories, with a deeper notion of uncertainty and natural approaches to problems.

Despite the convincing superiority of quantum theories with respect to classical probability models, a fundamental question is still open: Are cognitive processes governed by stochastic principles at all? To address this question, we will focus on the compatibility and expediency of stochastic approaches from the point of view of neuroscience research, and omit any involvement in philosophical discussions on the nature of human judgment and decision making.

Following Griffiths and colleagues (2010), the authors characterize probabilistic models of cognition as "top-down" or "functionfirst." Furthermore, P\&B espouse the philosophy that "neuroscience methods and computational bottom-up approaches are typically unable to provide much insight into the fundamental why and how questions of cognitive process" (sect. 1.2) and hence suggest the right modeling strategy as beginning with abstract (stochastic) principles and then reducing them into (deterministic) neural processes.

However, the assumption of a stochastic nature of cognition and behavior has severe consequences both mathematically and biologically.

1. Convergence of stochastic and deterministic results: Using a deterministic dynamic systems model, computational neuroscience and biophysics improved our understanding of neuronal processes in the last decades. Particularly, the models reproduced and predicted neurochemical and electrophysiological processes that were shown to induce alterations in the behavior of animals and human (Knowlton et al. 2012; Maia \& Frank 2011; Noori \& Jäger 2010). The sum of these theoretical models and their experimental validations suggests a deterministic relationship between neural processes and behavior. On the other hand, stochastic models of cognition assign to each behavioral output a proper random variable in a probability space. Therefore, a certain behavior could be characterized as a deterministic function of biological variations and a random variable simultaneously. This paradoxical duality requires the deterministic and stochastic functions to converge to the same behavioral outcome under given conditions. Consequently, the compatibility of the top-down stochastic approaches with biological findings and the possibility of a reduction into lower-level neural processes depend on the existence of appropriate convergence criteria, which have not been provided to date.

2. Interactions of neural processes and human behavior: From cellular dynamics to oscillations at the neurocircuitry level, numerous studies have identified biological processes that define/influence behavior (Morrison \& Baxter 2012; Noori et al. 2012; Shin \& Liberzon 2010). Therefore, cognition is a causal consequence of a series of biological events. In light of these investigations, a cognitive process of a stochastic nature inherited its "random" character from its underlying biology. In other words, the top-down reduction of the abstract stochastic principles into neural processes implies probabilistic dynamic behavior of neural systems at different spatiotemporal scales. However, the lack of theoretical or experimental models confirming the probabilistic nature of neural mechanisms challenges the proposed top-down strategy.

In conclusion, although the quantum probability theories significantly extend the application field of classical Bayesian theories for modeling cognitive processes, they do not address the general criticisms towards top-down modeling approaches. 
Commentary/Pothos \& Busemeyer: Can quantum probability provide a new direction for cognitive modeling?

\section{Quantum probability, intuition, and human rationality}

doi:10.1017/S0140525X12003081

Mike Oaksford

Department of Psychological Sciences, Birkbeck College, University of London, London WC1E 7HX, United Kingdom.

mike.oaksford@bbk.ac.uk

http://www.bbk.ac.uk/psyc/staff/academic/moaksford

Abstract: This comment suggests that Pothos \& Busmeyer (P\&B) do not provide an intuitive rational foundation for quantum probability $(\mathrm{QP})$ theory to parallel standard logic and classical probability (CP) theory. In particular, the intuitive foundation for standard logic, which underpins $\mathrm{CP}$, is the elimination of contradictions - that is, believing $p$ and not- $p$ is bad. Quantum logic, which underpins QP, explicitly denies non-contradiction, which seems deeply counterintuitive for the macroscopic world about which people must reason. I propose a possible resolution in situation theory.

One of the motivations underlying the psychology of human reasoning has been the assessment of human rationality: that is, comparing human reasoning performance with some standard of rationality provided by a normative theory, be it standard bivalent logic, Bayesian probability theory, expected utility theory, or quantum probability (QP) theory (Chater \& Oaksford 2012). Whether this is a proper function of psychological theories of reasoning has been debated within the pages of Behavioral \& Brain Sciences (Elqayam \& Evans 2011), but QP is a competitor to classical probability $(\mathrm{CP})$ theory precisely at the computational, normative level. Pothos \& Busmeyer $(\mathrm{P} \& \mathrm{~B})$ are quite explicit on this point when they observe that under a QP interpretation, the conjunction fallacy is not fallacious. This assertion makes sense only with the understanding that it conforms to the dictates of QP, considered as a normative theory of how people should reason. However, $\mathrm{P} \& \mathrm{~B}$ do not provide an account of why, intuitively, QP is rational. Rather, in section 5, "The rational mind," they concentrate on pointing out existing problems with the claims of CP, to provide an account of rationality.

Although as $\mathrm{P} \& \mathrm{~B}$ observe, there are problems for existing normative theories, there are very clear underlying intuitions about why they are rational. For standard bivalent logic, the primary intuition is that contradictions are bad-that is, it is irrational to believe both $p$ and not- $p$ (e.g., chalk is white and chalk is not white). If one follows the laws of logic, one will never fall into contradiction, and, therefore, logic can provide a rational standard. Similarly for probability theory, the primary intuition is that making bets that one is bound to lose is bad. If one follows the laws of probability in the Kolmogorov axioms, one will never make a bet one is bound to lose, and therefore it can provide a rational standard. $\mathrm{P} \& \mathrm{~B}$ provide no similar intuitive understanding of how QP is rational, even if this understanding is ultimately deficient in some respects, as they point out for CP. Rather, $\mathrm{P} \& \mathrm{~B}$ observe that QP is loosely like bounded rationality, and is consistent with the isotropic and the Quinean nature of human thought.

The lack of an intuitive grasp of rationality in QP relates to the closing sentence of section 5: "For the real, noisy, confusing, everchanging, chaotic world, QP is the only system that works in physics and, we strongly suspect, in psychology as well.” The world they describe here is actually the microscopic world of quantum events that supplies the underlying domain of QP that is, the world whose behaviour it is trying to describe. However, the world that provides the appropriate domain for logic and CP is a relatively stable macroscopic world of which we have varying states of knowledge. Insofar as the statements of these theories describe these worlds, they provide a theory of what these statements mean. There are obvious complexities here, but one requirement of a formalism is that, in Susan Haack's (1978) terminology, it should be capable of respecting the appropriate depraved semantics (Haack 1978, p. 188) - that is, people's intuitive understanding of a domain.
Logic and CP, to the extent they have a rational foundation, assume a world where events cannot both occur and not occur and where there are objective values. The extent to which one might be inclined to adopt QP is the extent to which one is willing to view the macroscopic world as being much more like the microscopic world than we have so far considered, hence it can provide an appropriate depraved semantics. One might also question whether the actual world of everyday experience is the "noisy, confusing, ever-changing, chaotic" place or whether this is the product of our situatedness within in it. However we view it, one should not underestimate the magnitude of the change in the conception of the macroscopic world underlying human cognition that QP entails.

For example, in quantum logic (Haack 1974), which underpins QP in the same way that classical logic underpins CP, the law of the excluded middle, or non-contradiction $(\operatorname{not}(p$ and not- $p))$, does not hold-that is, the intuitive rational basis of classical logic is explicitly denied. Therefore, in the microscopic world, quantum events can occur and not occur. But to embrace $\mathrm{QP}$ is - for some events at least, that is, when questions are incompatible - to require people to reject non-contradiction for some macroscopic events, which seems deeply counterintuitive. However, this may not be quite as counterintuitive as it seems.

Although there is no scope to develop the connections here in any detail, there is a semantic account more readily compatible with QP. This account is provided by situation theory (see Barwise \& Perry 1983; for a recent review, see Stojanovic 2012), in which situations - construed as something between the physical and the psychological, simplistically, the bit of world one can see that can include other people who might have a different perspective on the same situation - are the basic building blocks, and objects and regularities only emerge as uniformities against the background of the ever-changing situations in which we find ourselves. Joining such a world view with QP may provide an intuitive, rational handle on QP. For example, perspectival terms, such as "behind" (which are often assimilated to indexicals), reveal apparent breakdowns of non-contradiction. Therefore, for example, "the table is behind the sofa" may be true from my perspective in a situation, but it may be false from yours. "Perspectival relativity" is an important feature of situation semantics.

In summary, whereas one cannot help but be impressed by the empirical grasp of QP, its potential to provide an alternative rational foundation for human reasoning requires further consideration, as for some events, the failure of non-contradiction at the macroscopic level seems deeply counterintuitive. However, there are accounts of the semantics of natural language that appear compatible with QP, and those may provide a much needed intuitive foundation.

\section{What's the predicted outcome? Explanatory and predictive properties of the quantum probability framework}

\author{
doi:10.1017/S0140525X12003093
}

Timothy J. Pleskac, Peter D. Kvam, and Shuli Yu

Department of Psychology, Michigan State University, East Lansing, MI 48824.

pleskact@msu.edu, kvampete@msu.edu, yushuli@msu.edu www.msu.edu/ pleskact

Abstract: Quantum probability $(\mathrm{QP})$ provides a new perspective for cognitive science. However, one must be clear about the outcome the QP model is predicting. We discuss this concern in reference to modeling the subjective probabilities given by people as opposed to modeling the choice proportions of people. These two models would appear to have different cognitive assumptions. 
Commentary/Pothos \& Busemeyer: Can quantum probability provide a new direction for cognitive modeling?

What is sometimes required is not more data or more refined data but a different conception of the problem.

$$
\text { —-Roger Shepard (1987, p. 1318) }
$$

Shepard made this statement in arguing that a universal law of generalization could be had only by formulating it around stimuli existing in a psychological, not a physical, space. Only then, according to Shepard, could principles of generalization be established that were invariant to context. Pothos \& Busemeyer $(\mathrm{P} \& \mathrm{~B})$ are making a very similar argument. They contend that the probabilistic nature of cognition may adhere not to the assumptions of classical probability $(\mathrm{CP})$, but instead to the properties of quantum probability (QP).

Perhaps this is the reconceptualization that is needed. Phenomena that were errors according to $\mathrm{CP}$, such as violations of the conjunction rule, order effects, and violations of the sure-thing principle, are consistent within the structural constraints of QP. Moreover, a quantum account can offer a more parsimonious explanation of the phenomena. Take, for example, the Linda problem. The typical explanation of this problem has been the representativeness heuristic in which people substitute similarity for probability (Kahneman 2003). In comparison, similarity is implicit in the QP calculation (reflected in the angle of the initial state vector relative to the bases).

The QP model of the Linda problem and other judgments of subjective probability (Busemeyer et al. 2011) are intriguing because they describe how people actually assign probabilities. However, P\&B use the same QP modeling framework to describe both the process of making subjective probabilities (e.g., the Linda problem) and the process of making a choice (e.g., Clinton vs. Gore honesty choice). This failure to distinguish between subjective probabilities and choice proportions presents a concern. To illustrate, consider another version of the Linda problem that researchers have used, in which people have to identify which of two statements is more probable (e.g., "Linda is a bank teller" or "Linda is a bank teller and is active in the feminist movement") (e.g., Tversky \& Kahneman 1983). When applied to choice proportions, QP is used to predict the objective probability of choosing either statement by projecting onto a basis vector. However, in modeling subjective probabilities, the model uses the same operator; therefore, it is as if people have direct access to the probability of entering into that state. Is the same cognitive system used for these different responses? To suppose these two models are of the same class seems to be an error. To do so would be to assume that subjective ratings of probability are the same as the probability of choosing a particular response.

The distinction between modeling the probability distributions over behaviors and modeling probability judgments is important. In the Linda problem, the probability judgment response is only one of many responses in which the conjunction fallacy is observed. The primary data, for example, were in terms of ranking eight possible hypotheses in order from least to most likely (Tversky \& Kahneman 1983). We also know that the extent to which people commit the conjunction fallacy changes based on the format of the response, such as with bets (Tversky \& Kahneman 1983), or with frequency formats (Mellers et al. 2001). It would seem that a more complete process level account of the conjunction fallacy would explain how these different response formats (or measurements) work, and would inform any model predicting the effect.

A more general comment regarding the thesis of the article is that quantum theory can provide a "better" probabilistic framework for cognition. The flexibility of QP theory may become a concern, as skeptics might argue that it lacks specific predictions. However, many predictions may be overlooked simply because they reflect common sense or because the theory is new enough that due scrutiny has not been afforded to uncover psychological inferences from Gleason's theorem or the Hilbert space. Take, for example, the prediction that projecting a state vector to a lower dimensional subspace will result in lost amplitude. This does appear to be consistent with the famous Korea/China asymmetry in similarity judgments (Tversky 1977), and certainly similar predictions can be made in other areas, such as comparing emotions of different dimensionality.

Perhaps the more difficult aspect is explanation. This seems tricky, as we are asked to take a different interpretation of a cognitive system under QP. For example, under a classical view, a cognitive system, at any given time point, is in a particular state. However, in QP we have superposition meaning a cognitive system is in no state at all! This idea would appear to change our ability to point to information as a causal mediating mechanism, as well as our understanding of the nature of mental events themselves. It also seems to change the very nature of what it means to explain cognition, as superposition seems almost uninterpretable. Hughes (1989) suggests that an explanation in terms of QP is a structural explanation. That is, it shows how the stochastic nature of cognition and its probability functions can be modeled using Hilbert spaces.

Is a structural explanation sufficient for psychologists? This concern and the larger set of issues it raises may not come as a particular surprise, as QP theory was originally designed only to predict the probability of outcomes of physical events. The challenge of modeling subjective judgments and mental processes is obviously a new challenge for QP, and, therefore, it may simply be that more work is needed to attune the QP framework to address psychological, rather than physical, problems. Even so, we suspect that the success of QP in cognitive modeling will depend largely on its reinterpretation, reapplication, and resulting predictive power rather than its narrative explanation.

\section{ACKNOWLEDGMENT}

A grant from the National Science Foundation (0955410) supported this work.

\section{If quantum probability = classical probability + bounded cognition; is this good, bad, or unnecessary?}

doi:10.1017/S0140525X12003172

\section{Tim Rakow}

Department of Psychology, University of Essex, Wivenhoe Park, Colchester CO4 3SQ, United Kingdom.

timrakow@essex.ac.uk

http://www.essex.ac.uk/psychology/department/people/rakow.html

Abstract: Quantum probability models may supersede existing probabilistic models because they account for behaviour inconsistent with classical probability theory that are attributable to normal limitations of cognition. This intriguing position, however, may overstate weaknesses in classical probability theory by underestimating the role of current knowledge states and may under-employ available knowledge about the limitations of cognitive processes. In addition, flexibility in model specification has risks for the use of quantum probability.

The case for using quantum probability that Pothos \& Busemeyer $(\mathrm{P} \& \mathrm{~B})$ present seems to be that quantum probability - like classical Bayesian probability - supports a probabilistic approach to cognitive modelling, but-unlike classical probability-predicts behaviour consistent with the attributes of bounded cognition such as the normal limitations in memory, processing capacity, and attentional control. However, before accepting this intriguing proposal, there are questions to consider. Is classical probability theory truly inconsistent with behavioural phenomena attributable to bounded cognition? Do we need alternatives to existing models that already incorporate insights concerning bounded cognition? Are there dangers in a unified quantum probability framework that subsumes classical probability and bounded cognition? 
One feature of assigning probabilities is that uncertainty can be aleatory or epistemic. Aleatory uncertainty derives from randomness in determining outcomes, whereas epistemic uncertainty reflects incomplete knowledge of outcomes and their precipitating factors. For example, prior to flipping a coin I assign Prob(Heads) $=0.5$ (aleatory uncertainty); however, I still assign Prob(Heads $)=0.5$ if the coin has been flipped but is covered (epistemic uncertainty). Often, both kinds of uncertainty are implied in probability assessments: someone may be uncertain whether he or she is employed at time $t$ both because there is some randomness in determining employment termination, and because he or she does not know what managerial decisions have occurred. Importantly, from a classical probability perspective, probabilities are always conditional on current knowledge; and, consequently, some of P\&B's illustrations are perhaps not so puzzling for classical probability theory as they suggest. My state of knowledge does differ according to whether someone asks me if Gore is honest before or after asking me whether Clinton is honest. In one case my knowledge includes the information "I have just been asked to assess Clinton's honesty," and in the other case it does not.

Moreover, as per the standard explanations of order effects that $\mathrm{P} \& \mathrm{~B}$ acknowledge, not only does my assessment of Gore alter when comparison with Clinton is encouraged, but also my classification for "honesty" may change (e.g., the category boundaries for honesty move). If the two questions about Gore refer to different events (i.e., alternative conceptions of honesty), there is little puzzlement for classical probability theory when the probabilities of "yes" differ between them. Therefore, if classical probability theory informed by the bounded nature of cognition can accommodate order effects (and other effects described by $\mathrm{P} \& \mathrm{~B}$ ) what does it gain us to have an alternative - arguably less intuitive - account from quantum probability theory? Numerous probabilistic cognitive models (including those previously championed by $\mathrm{P} \& \mathrm{~B}$ ) already incorporate features of bounded cognition, including limitations in memory (Bush \& Mostellar 1955), attentional control (Birnbaum 2008), or processing capacity (Tversky 1972). P\&B seem to be proposing models that bypass the need for instantiating definable psychological processes within cognitive models, because quantum probability can account for phenomena usually explained through bounded cognition by another route. Seemingly, in quantum probability models of cognition, all the "work" is done by a probability theory with the flexibility to account for many findings, without incorporating cognitive theory in the model. How does this advance our understanding of psychological processes?

$\mathrm{P} \& \mathrm{~B}$ propose that: "superposition appears an intuitive way to characterize the fuzziness (the conflict, ambiguity, and ambivalence) of everyday thought" (sect. 1.1). Surely, we already have an intuitive explanation for the fuzziness of everyday thought: thinking includes difficult assessments of unpredictable events (aleatory uncertainty), under conditions of incomplete knowledge (epistemic uncertainty), where often only a subset of the potentially available information is used (bounded cognition). What does "superposition" bring to the table if we already accept that information is incomplete? How does "time evolution" differ from acknowledging that knowledge states can change rapidly? What does "interference" add to the simple observation that attention shifts between the reasons or factors that underpin assessments?

I acknowledge that it is a weak argument against quantum probability formulations of cognitive models that they offer no more than existing accounts: there is no particular reason to disfavour a framework just because other frameworks already exist, and arguably there is always room for another account that addresses a different level of explanation. In other words, if quantum probability = classical probability + bounded cognition, then quantum probability theory deserves its place. That said, it seems odd to propose a class of cognitive models that, if I understand P\&B correctly, need fewer components that relate to cognitive processes because quantum probability theory already predicts phenomena that those cognitive processes explain.

Additionally, there are potential dangers associated with a framework that subsumes an earlier one. For compatible questions, quantum probability reduces to classical probability, and, therefore, $\mathrm{P} \& \mathrm{~B}$ rightly assert that the modeler determines a priori which questions are (in) compatible (sect. 2.2). However, just how a priori is a priori? If incompatibility is determined prior to specifying any formal models but after summarising the data, we risk the following unsatisfactory state of affairs:

1. How do we know that these questions are incompatible?

2. Because classical probability theory was violated.

3. Why does the model predict violations of classical probability theory?

4. Because these questions are incompatible.

If incompatibility is determined prior to data collection, this problem does not disappear, because we already know much about predicting the kinds of situations in which order effects, and other violations of classical probability theory, occur. If the incompatibility of questions cannot be specified in some way that is fully independent of the data, then quantum probability models risk benefitting from the mother of all free parameters: explaining data consistent with classical probability, and data that violate classical probability theory. Similar concerns about the flexibility in model specification afforded by quantum probability arise in the example of modeling the Linda problem. The assumption behind placing the quantum probability subspaces was that feminism is "largely uninformative to whether a person is a bank teller or not" (sect. 3.1). However, the standard account that Linda is representative of a feminist but unrepresentative of a bank teller might imply that people perceive that feminism is informative (radical feminism being inconsistent with bank telling). Therefore, for the legitimate application of quantum probability to cognitive modeling, we may also need clear guidance on the a priori specification of subspaces. Otherwise, without such checks and balances on the specification of quantum probability models, although, seemingly, such models could account for everything, they would explain nothing (Glöckner \& Betsch 2011).

\section{Quantum probability, choice in large worlds, and the statistical structure of reality}

doi:10.1017/S0140525X1200310X

Don Ross ${ }^{\mathrm{a}}$ and James Ladyman ${ }^{\mathrm{b}}$

${ }^{a}$ School of Economics, University of Cape Town, Cape Town, South Africa; ${ }^{\mathrm{b}}$ Department of Philosophy, University of Bristol, Bristol BS81TB, United

Kingdom.

don.ross@uct.ac.za

http://uct.academia.edu/DonRoss

james.ladyman@bristol.ac.uk

http://bristol.ac.uk/philosophy/department/staff/JL/jl.html

Abstract: Classical probability models of incentive response are inadequate in "large worlds," where the dimensions of relative risk and the dimensions of similarity in outcome comparisons typically differ. Quantum probability models for choice in large worlds may be motivated pragmatically-there is no third theory-or metaphysically: statistical processing in the brain adapts to the true scale-relative structure of the universe.

Pothos \& Busemeyer (P\&B) propose quantum probability (QP) theory as a general axiomatic structure for modeling incentivized response (choice). Experiments in the Kahneman-Tversky tradition suggest that the relationship between information and human behavioral choice does not respect the axioms of classical probability (CP) theory, notably monotonicity. Furthermore, 
sequences of choices violate the classical law of total probability. Modelers have accommodated these departures from CP by hypothesizing heuristics - procedures that are specially adapted to specific types of situations-but in so doing postpone developing a general, testable theory that formally integrates aspects of cognition, for example, similarity judgment and assessments of risk. The probability model developed by quantum physicists is a formal alternative to $\mathrm{CP}$ that handles many of the recalcitrant phenomena, and can reproduce successful predictions of models based on classical probability in the classical limit.

The premise that the CP-based theory of decision - which is better characterized as a theory of incentive response - has been refuted by experimental evidence is questionable. The mathematical modeling of risk and the econometric estimation of responses to changes in structural parameters of risk models, are currently enjoying technical innovation, starting with Hey and Orme (1994) and surveyed in Harrison and Rutstrom (2008). There is evidence that unintended constraints derived from less sophisticated risk models may be implicated in a range of phenomena that have been thought to imply use of special-purpose heuristics (e.g., Allais violations, loss aversion, hyperbolic discounting; see Andersen et al. 2011; Harrison \& Rutström 2009). This research might be taken as supporting the model of Oaksford and Chater (2007) on a descriptive rather than a normative interpretation, with its ambition to underwrite optimization relativized to heterogeneous preference structures. As $\mathrm{P} \& \mathrm{~B}$ note, the Bayesian rationality model relies on objective value maximization rather than subjective utility maximization to handle Dutch book problems, and this reliance is not acceptable to economists. Global optimality of decision procedures is surrendered once one allows that people's risk preferences are heterogeneous and circumstance specific, and that utility functions are often rank dependent. However, this undermines confidence in CP-based Bayesian models only as accounts of decisions in what Binmore (2009) calls "large worlds"; they may yet be the right models for "small world" problems. The idea that any empirically adequate general theory of decision might exist for large worlds is purely conjectural.

However, $\mathrm{P} \& \mathrm{~B}$ 's main point about Bayesian rationality is more interesting. CP derives some of its support from philosophers and others imagining that thought should work well in large worlds. Once we are wondering about large worlds, we are in the territory of metaphysics. Suppose for the sake of argument that $\mathrm{P} \& \mathrm{~B}$ are right that $\mathrm{QP}$ offers the correct general theory of human incentive response. This would invite the question as to whether there is some general feature of the world that explains why both fundamental physical structure and fundamental cognitive structure follow QP rather than CP. The more modest philosophy would be to regard QP in psychology as just a placeholder, which we borrow from physics if we decide we should give up on CP because, for now, it is the only formally worked out alternative.

As $\mathrm{P} \& \mathrm{~B}$ note, one possible motivation for the immodest philosophy is the hypothesis that cognition follows QP axioms because the brain's physical structure allows it to perform quantum computation. But this is mere speculation. Another suggestion is that our view of $\mathrm{CP}$ as the default account results from a failure of metaphysical imagination that has been observed in other areas of science. The failure in question is the assumption that there must be a general account of reality that applies to all scales of structural complexity. This is a prejudice derived from treating human mechanical interactions with medium-sized stable "things" as the structural field we should universally project. Many philosophers presume a kind of atomism that is inconsistent with quantum physics (Ladyman \& Ross 2007). Arguably, the philosophy underlying CP-based accounts of cognition is what Russell (1921; 1918-1924/1956, pp. 177-343) called logical atomism. Does denial of physical atomism put stress on logical atomism, or this simply sloppy homology?
Ladyman and Ross (2007) explain and defend the scale relativity of ontology. By this we mean that the persistent patterns that compress information and thus support generalizations and outof-sample predictions - in a word, existents - arise at different scales that cross-classify reality and do not reduce to one another. Conscious, deliberate theoretical reasoning of the kind modeled by philosophers, we suggest, ignores scale relativity by generalizing the kind of atomistic decomposition that generally works well in everyday mechanical manipulations. P\&B's hypothesis suggests an interesting twist. Most cognition is not deliberate theorizing; it is statistical data processing by neural networks, converging on relatively stable perceptions and expectations by drift diffusion rather than deduction. If the world does not parse neatly into components on a single scale, why should nervous systems evolved to compress and store information about that world incorporate a false restriction to the effect that all relations of similarity and difference, understood in terms of structural distance, be representable on a single scale? This perspective helps us understand the difference between the small words where Bayesian reasoning works and the large worlds where it does not: a small world is a restriction to one scale.

Challenges remain for the application of QP to cognition. The formalism and the physics may not come apart as neatly as $\mathrm{P} \& \mathrm{~B}$ suggest. Quantum mechanics has a well-confirmed law of time evolution, the Schrödinger equation, governing systems at least when they are not being measured. $\mathrm{P} \& \mathrm{~B}$ offer no analogous law of time evolution of cognitive states. Schrödinger time evolution is based on the assignment of a Hamiltonian representing the energy of the system, and we lack any analogue of energy as well. Quantum mechanics involves effects where Plank's constant - the quantum of action - is not negligible and the limit in which the predictions of classical mechanics are reached is that in which $\mathrm{h}$ goes to zero. There is no analogously well-defined limit in the application to cognition.

\section{Physics envy: Trying to fit a square peg into a round hole}

\section{doi:10.1017/S0140525X12003160}

\section{James Shanteau ${ }^{\mathrm{a}}$ and David J. Weiss ${ }^{\mathrm{b}}$ \\ ${ }^{a}$ Department of Psychology, Bluemont Hall, Kansas State University, Manhattan, KS 66506; ' Department of Psychology, California State University, Los Angeles, CA 90032. \\ shanteau@k-state.edu \\ dweiss@calstatela.edu \\ www.ksu.edu/psych/cws \\ www.davidjweiss.com}

Abstract: Pothos \& Busemeyer (P\&B) argue that classical probability (CP) fails to describe human decision processes accurately and should be supplanted by quantum probability. We accept the premise, but reject P\&B's conclusion. CP is a prescriptive framework that has inspired a great deal of valuable research. Also, because CP is used across the sciences, it is a cornerstone of interdisciplinary collaboration.

Although Pothos \& Busemeyer (P\&B) make a noteworthy effort to apply quantum probability $(\mathrm{QP})$ theory to what they term classical (Bayesian) probability $(\mathrm{CP})$ theory, we feel that their effort has fallen short on three grounds.

1. $\mathbf{C P}$ as a straw man. First, they cast $\mathrm{CP}$ as a theory of the mind. For Ward Edwards, the founder of research on behavioral decision making and the first to use the term "subjective probability," as well as for Kahneman \& Tversky and their adherents, CP served as a point of comparison. Although one might argue that sensible decisions ought to follow CP, judgment and decision making (JDM) research has largely been about how decisions made by people do not. To use Baron's (2004) terminology, CP may be useful as a prescriptive theory of behavior, but not as a descriptive theory. 
Commentary/Pothos \& Busemeyer: Can quantum probability provide a new direction for cognitive modeling?

The appeal of CP to most psychologists is quite different. We seek a way to describe uncertainty. Uncertainty is a central construct that all sciences need to address. If there is to be any hope of communicating across disciplines (or subfields of psychology), we need to use that common construct.

2. Distorting behavior to accommodate mathematical requirements. Second, in order to define probabilities, $\mathrm{P} \& \mathrm{~B}$ readily dichotomize a continuum. For example, they discuss two questions: (1) Is the person happy? (2) Is the person employed? They treat these questions as if they are equivalent, both having yes-no answers, and assign probabilities to the responses. The focus is on how the answers interplay: happiness might be more probable if a person is employed (because he or she has money) or less probable (because she hates her job).

However, happiness is a continuum, not a dichotomy. Translating from mathematical exercises into real research questions allows this distinction to be seen clearly. For example, one might try a manipulation to change a person's degree of happiness. The change could be measured, perhaps, by asking, "How happy are you now?" at various times. The amount of change, as opposed to whether there has been a change of status, is important.

Dichotomizing not only loses information (MacCallum et al. 2002), it can seriously distort substantive inferences. The latter danger is illustrated in the critique of a respected index of clinically significant change (Jacobson et al. 1999) by Weiss et al. (2009). In contrast, employment has only two, or maybe a few (part-time, or multiple-jobs) states. Therefore, it makes sense to use dichotomized responses in asking a subject about employment ("did the manipulation change your employment status?").

3. Complexity beyond the needs of the research domain. Third, although the level of mathematical sophistication underlying QP is admirable, we view it as a case of overkill. As argued by fourteenth century logician and Franciscan friar William of Ockham, "entities should not be multiplied unnecessarily." This recommendation is now commonly known as "Occam's Razor." Ultimately, of course, whether complexity is excessive is a subjective matter. It is our opinion that $\mathrm{P} \& \mathrm{~B}$ have developed an unnecessarily complicated alternative to $\mathrm{CP}$.

Traditional CP is notable for its simplicity. Augmented with a Bayesian perspective, it has been a remarkably valuable tool for stimulating JDM researchers ranging from Edwards in the 1950s and 1960s, to Kahneman and Tversky in the 1970s and 1980s, to Gigerenzer and colleagues in the 1990s and 2000s, to the recent Behavioral \& Brain Sciences article by Elqayam and Evans (2011) arguing for a distinction between normative systems and competence theories (see Brase \& Shanteau 2011).

Young (1996) explained to the Psychometric Society why he was no longer interested in building increasingly more sophisticated algorithms for multi-dimensional scaling (MDS). Young had been one of the pioneers in developing MDS tools. His arguments can be summarized by the following quotes from his presentation: "Application to problems in Psychology and Social Sciences have gotten lost in the process of developing more complex methods and models... The methods and models have gotten much more complicated than users can understand...We are too focused on mathematics at the expense of usefulness." We believe that Young's comments on MDS are equally applicable to the article by P\&B.

4. Physics Envy. There has been ongoing discussion among behavioral scientists regarding "Physics Envy" (Mirowski 1992; Sapolsky 1997; Schabas 1993). In part, recent discussions in economics (Lo \& Mueller 2010) have been motivated by the role of socalled "quants" in the 2007 financial meltdown. As Malkiel noted, whereas "physical models can provide an accurate description of reality...financial models, despite their mathematical sophistication, can at best provide a vast oversimplification of reality" (Malkiel 2011, p. 1)

Although we are not as pessimistic about the role of mathematical models in psychology, we share the concern about using physics as a standard against which to evaluate theories in psychology. The issue, according to Lo and Mueller, is that "human behavior is not nearly as stable as and predictable as physical phenomena" (Lo \& Mueller 2010 , p. 13). Therefore, tools appropriate for advances in physics may not be nearly as useful in psychology.

\section{Realistic neurons can compute the operations needed by quantum probability theory and other vector symbolic architectures}

\section{doi:10.1017/S0140525X12003111}

\section{Terrence C. Stewart and Chris Eliasmith}

Centre for Theoretical Neuroscience, University of Waterloo, Waterloo, ON N2L 3G1, Canada.

tcstewar@uwaterloo.ca

celiasmith@uwaterloo.ca

http://ctn.uwaterloo.ca/

Abstract: Quantum probability (QP) theory can be seen as a type of vector symbolic architecture (VSA): mental states are vectors storing structured information and manipulated using algebraic operations. Furthermore, the operations needed by QP match those in other VSAs. This allows existing biologically realistic neural models to be adapted to provide a mechanistic explanation of the cognitive phenomena described in the target article by Pothos \& Busemeyer (P\&B).

If we are to interpret quantum probability $(\mathrm{QP})$ theory as a mechanistic cognitive theory, there must be some method whereby the operations postulated by QP are implemented within the human brain. Whereas this initially seems like it would require some sort of large-scale quantum effect in the brain, the target article notes that "the relevant mathematics is simple and mostly based on geometry and linear algebra" (sect. 1.2). No special quantum physics effects are needed. If we can show how neurons can compute these operations, then we can interpret QP as making strong claims about how brains reason, rather than merely acting as a novel behavioral description of the results of cognitive processing.

Interestingly, there is already a family of cognitive models that make use of geometry and linear algebra to describe cognitive mechanisms, and these share many features with QP. Vector symbolic architectures (VSAs; Gayler 2003) use high-dimensional vectors to store structured information, and use algebraic operations to manipulate these representations. The closest match to QP among VSAs is holographic reduced representations (HRRs; Plate 2003). As with QP, vectors are added to combine information and the dot product is used to evaluate similarity. For example, if there is one vector for HAPPY and another for UNHAPPY, the current mental state representation might be 0.86 HAPPY +0.5 UNHAPPY, representing a state more similar to HAPPY than to UNHAPPY. While the notation is different, this is identical to the $|\Psi>=a|$ happy $>+b \mid$ unhappy $>$ example in the target article (sect. 2.1; Fig. 1a). If EMPLOYED and UNEMPLOYED are other vectors that are similar to HAPPY and UNHAPPY, respectively, then we get Figure $1 b$.

For the case of Figure 1c, HRRs and QP differ. Rather than using tensor products like |happy> $\otimes \mid$ employed $>$ (sect 2.2.2), HRRs use circular convolution (HAPPY $\circledast$ EMPLOYED). This was specifically introduced by Plate as a compressed tensor product: an operation that gives the same effects as a tensor product, but that does not lead to an increase in dimensionality. To explain this, consider the transition from Figure $1 \mathrm{~b}$ to $1 \mathrm{c}$ in the target article. In the first case, we are dealing with a twodimensional space, and in the second it is a four-dimensional space. How is this represented in the brain? How do neurons dynamically cope with changing dimensionality? Are different neurons used for different cases? For HRRs, these concerns are 
Commentary/Pothos \& Busemeyer: Can quantum probability provide a new direction for cognitive modeling?

addressed by having a fixed (but large) dimensionality for all representations. If HAPPY is a particular 500-dimensional vector, and EMPLOYED is a different 500-dimensional vector, then HAPPY $\circledast$ EMPLOYED gives a new 500-dimensional vector (a tensor product would give a 250,000-dimensional vector). Importantly, in high-dimensional spaces, the newly created vector is highly likely to be (almost) orthogonal to the original vectors. This gives a close approximation to all of the required orthogonality requirements mentioned in the target article, but does not lead to an unlimited explosion of dimensions as representations get more complicated. As we have shown elsewhere, adult human vocabularies fit well within 500 dimensional spaces (Eliasmith, in press).

Given cognitive theories expressed in terms of vector symbolic architectures, we have created large-scale neural models that implement those theories. In particular, we use the neural engineering framework (NEF; Eliasmith \& Anderson 2003), which gives a principled method for determining how realistic spiking neurons can represent vectors, how connections between neurons can implement computations on those vectors, and how recurrent connections can be used to provide memory and other dynamics. This allows us to turn abstract descriptions of cognitive processing into specific brain mechanisms, connecting a plethora of neural data (functional magnetic resonance imaging [fMRI], electroencephalograms [EEG], single cell recordings) to cognitive function.

In the NEF, distributed representations of vectors are made by generalizing the standard notion of each neuron having a particular preferred direction vector (e.g., Georgopoulos et al. 1986). Whereas Hebbian learning rules can be used to adjust connection weights, we can also directly solve for the desired connection weights, as this kind of distributed representation allows a much larger range of functions to be computed in a single layer of connections than is seen in typical connectionist models. This makes it straightforward to create models that accurately compute linear operations (such as the dot product), and even more complex functions such as a full 500-dimensional circular convolution. These models are robust to neuron death and exhibit realistic variability in spiking behavior, tuning curves, and other neural properties.

Although these techniques have not yet been used on the specific tasks and theories presented in the target article, all of the operations mentioned in the article have been implemented and scaled up to human-sized vocabularies (e.g., Eliasmith 2005; Stewart et al. 2011). Furthermore, we have shown how to organize a neural control structure around these components (based on the cortex-basal ganglia-thalamus loop) so as to control the use of these components (e.g., Eliasmith, in press; Stewart \& Eliasmith 2011). This architecture can be used to control the process of first projecting the current state onto one vector (HAPPY) and then on to another (EMPLOYED), before sending the result to the motor system to produce an output. These neural models generate response timing predictions with no parameter tuning (e.g., Stewart et al. 2010), and show how the neural implementation affects overall behavior. For example, the neural approximation of vector normalization explains human behavior on list memory tasks better than the ideal mathematical normalization (Choo \& Eliasmith 2010).

Although the NEF provides a neural mechanism for all of the models discussed in the target article, it should be noted that this approach does not require Gleason's theorem, a core assumption of QP (sect. 4.3). That is, in our neural implementations, the probability of deciding one is HAPPY can be dependent not only on the length of the projection of the internal state and the ideal HAPPY vector, but also on the lengths of the other competing vectors, the number of neurons involved in the representations, and their neural properties, all while maintaining the core behavioral results. Resolving this ambiguity will be a key test of QP.

\section{Why quantum probability does not explain the conjunction fallacy}

\section{doi:10.1017/S0140525X12003123}

Katya Tentori ${ }^{\mathrm{a}}$ and Vincenzo Crupi ${ }^{\mathrm{b}}$

${ }^{a}$ Center for Mind/Brain Sciences (CIMeC), Department of Psychology and Cognitive Sciences (PSC), University of Trento, Palazzo Fedrigotti, Corso Bettini, n. 31, 38068 Rovereto, Italy; ${ }^{\mathrm{b} D i p a r t i m e n t o ~ d i ~ F i l o s o f i a ~ e ~ S c i e n z e, ~}$ dell'Educazione, Via S. Ottavio 20, 10124 Torino, Italy.

katya.tentori@unitn.it

vincenzo.crupi@unito.it

http://www.unitn.it/en/cimec/11750/katya-tentori

Abstract: We agree with Pothos \& Busemeyer (P\&B) that formal tools can be fruitfully employed to model human judgment under uncertainty, including well-known departures from principles of classical probability. However, existing findings either contradict P\&B's quantum probability approach or support it to a limited extent. The conjunction fallacy serves as a key illustration of both kinds of problems.

Pothos \& Busemeyer's (P\&B's) argument in favor of a quantum probability (QP) approach to cognitive modeling relies on the following premises:

1. A large amount of empirical findings accumulated in the last 40 years shows that human judgment often violates key aspects of classical probability $(\mathrm{CP})$ theory.

2. Heuristic-based approaches, although interesting, are often limited in their applicability and explanatory scope.

3. It is possible to model probability judgment on the basis of formal tools, and use these to re-express and sharpen popular heuristics.

We agree with $\mathrm{P} \& \mathrm{~B}$ on all the mentioned assumptions. However, we depart from $\mathrm{P} \& \mathrm{~B}$ in our assessment of the potential of their QP approach for achieving a better understanding of human judgments under uncertainty. We will illustrate our perspective with reference to the conjunction fallacy $(\mathrm{CF})$ (Tversky \& Kahneman 1982; 1983). The CF plays a key role in P\&B's argument because $\mathrm{P} \& \mathrm{~B}$ claim that this prominent violation of $\mathrm{CP}$ laws has a natural and straightforward explanation in their QP approach. In what follows, we will illustrate two main problems that arise with regard to $\mathrm{P} \& \mathrm{~B}$ 's interpretation of the CF results.

The first problem is that the QP approach is contradicted by empirical data. To begin with, it is unable to accommodate double conjunction fallacies (e.g., the mile run scenario in Tversky \& Kahneman 1983, p. 306) - that is, all those situations in which $h_{1} \wedge h_{2}$ is ranked over each of $h_{1}$ and $h_{2}$ appearing in isolation (Busemeyer et al. 2011, p. 202). Several single conjunction fallacy results are also demonstrably inconsistent with the QP approach. For example, suppose that, given some evidence $e$, the most likely statement has to be chosen among three, namely, a single hypothesis $h_{1}$ and two conjunctions, $h_{1} \wedge h_{2}$ and $h_{1} \wedge \sim h_{2}$, as in the following scenario:

$\mathrm{K}$. is a Russian woman.

Which of the following hypotheses

do you think is the most probable?

$-\mathrm{K}$. lives in New York.

$-\mathrm{K}$. lives in New York and is an interpreter.

$-\mathrm{K}$. lives in New York and is not an interpreter.

Tentori et al. (2013) observed that $P\left(h_{2} \mid e \wedge h_{1}\right)<P\left(\sim h_{2} \mid e \wedge h_{1}\right)$ for the majority of participants. One can just as safely assume that $P\left(h_{2} \mid e\right)<P\left(\sim h_{2} \mid e\right)$, as clearly only a tiny fraction of Russian women are interpreters. On these assumptions, the QP account of the conjunction fallacy demonstrably predicts that the judged probability of $h_{1} \wedge h_{2}$ must be lower than that of $h_{1} \wedge \sim h_{2}$ (see Fig. 1), and, therefore, that fallacious choices for $h_{1} \wedge h_{2}$ must be less than those for $h_{1} \wedge \sim h_{2}$.

However, in contrast to this prediction, a significant majority $(70 \%)$ of the fallacious responses in the Russian woman scenario 
Commentary/Pothos \& Busemeyer: Can quantum probability provide a new direction for cognitive modeling?

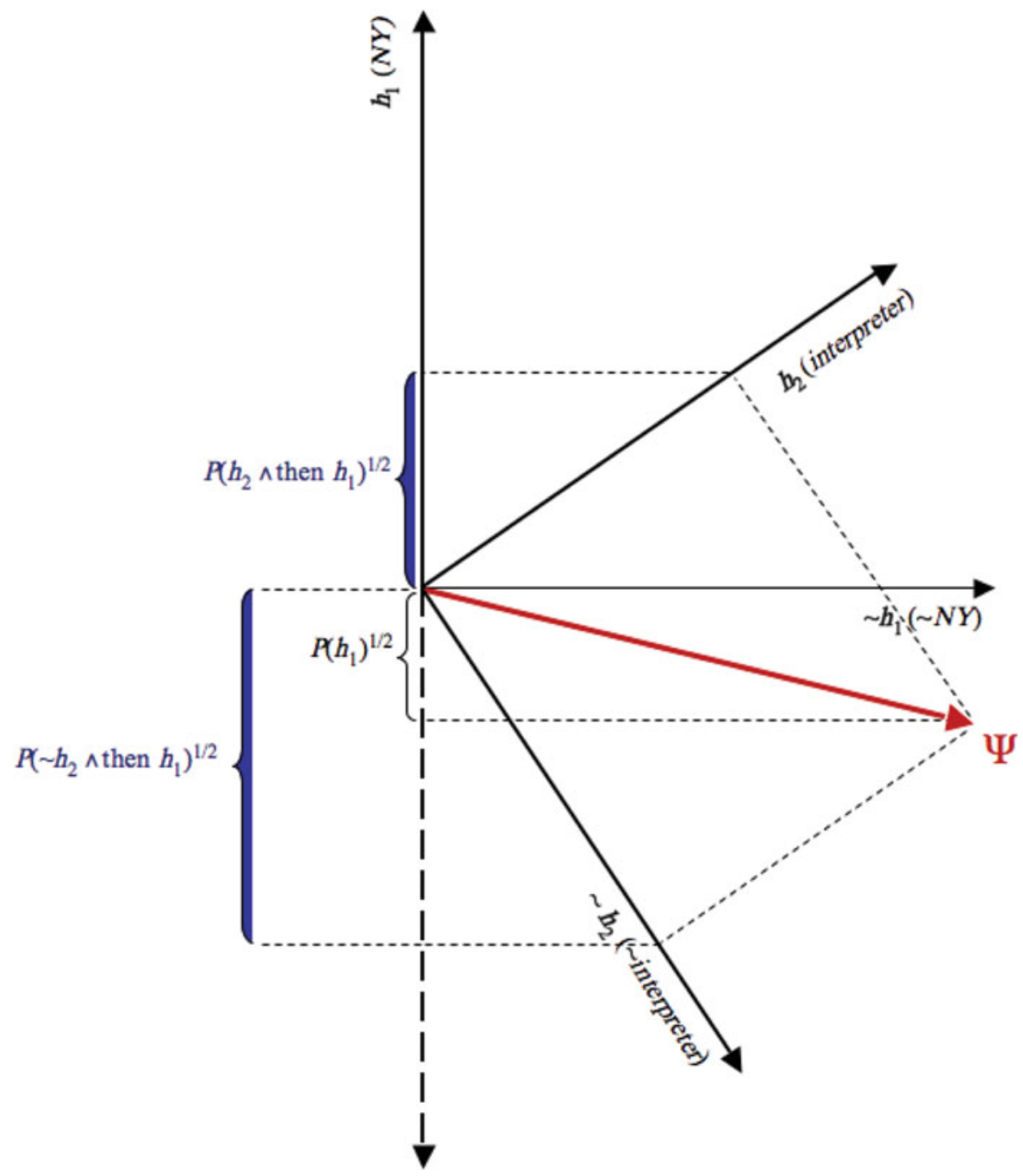

Figure 1 (Tentori \& Crupi). A QP representation of the Russian woman scenario. To simplify notation, $P$ is taken to already encode the evidence $e$ (Russian). In line with participants' judgment, the basis vectors are displayed as to imply $P\left(h_{2} \mid h_{1}\right)<P\left(\sim h_{2} \mid h_{1}\right)$, whereas the position of the state vector implies $P\left(h_{2}\right)<P\left(\sim h_{2}\right)$, as clearly only a tiny fraction of Russian women are interpreters. Under these conditions, if we assume - as it seems plausible - that $P\left(h_{1}\right) \geq P\left(h_{2}\right)$, the QP approach does not allow for the conjunctive probability of $h_{1}$ and $h_{2}$ to rank higher than the single conjunct $h_{1}$. However, the QP approach yields the wrong prediction even if we assume $P\left(h_{1}\right)<P\left(h_{2}\right)$. As the figure illustrates, in this case, contrary to the participants' judgment, it is the conjunctive probability of $h_{1}$ and $\sim h_{2}$, not that of $h_{1}$ and $h_{2}$, which must rank highest.

concerned $h_{1} \wedge h_{2}$ rather than $h_{1} \wedge \sim h_{2}$. More generally, one can prove that, for the QP account, if $P\left(h_{2} \mid e\right) \leq P\left(h_{3} \mid e\right)$ and $P\left(h_{2} \mid\right.$ $\left.e \wedge h_{1}\right) \leq P\left(h_{3} \mid e \wedge h_{1}\right)$, the judged probability of the conjunction $h_{1} \wedge h_{2}$ on the assumption of $e$ cannot be higher than that of $h_{1} \wedge h_{3}$. In a series of four experimental studies (which employed different elicitation procedures, experimental designs, classes of problems, and content), Tentori et al. (2013) documented a robust pattern of results inconsistent with this implication of the QP model, as well as with other recent proposals, such as the averaging (Nilsson et al. 2009) and the random error (Costello 2009) hypotheses, which imply that CF rates should rise as the perceived probability of the added conjunct does. The results supported a different explanation of the CF, based on the notion of inductive confirmation (Crupi et al. 2008). Much like the QP approach, this explanation relies on a well-defined formalism (Bayesian confirmation theory) while avoiding the limitations (e.g., post-hoc parameters) that P\&B (sect. 4.1) ascribe to other Bayesian models.

The second problem is that, even when logically consistent with the empirical data, $P \& B$ 's treatment nonetheless receives limited support. The QP modeler is typically left with a number of choices that are unconstrained by the model itself. Lacking independent and clearly defined empirical input, the modeling exercise does not achieve explanatory relevance. The Linda scenario serves as an illustration. For their QP approach to account for the reported judgment $P($ bank teller $\wedge$ feminist $)>P($ bank teller $), P \& B$ need to make various assumptions (sect. 3.1) on the angle between the basis vectors, as well as on the position of the state vector. Some of these assumptions are uncontroversial. Others are quite subtle, however, and have non-trivial consequences. As the left column of Figure 2 shows, keeping basis vectors equal, a small shift in the position of the state vector is enough to reverse the predicted ranking between $P($ bank teller $\wedge$ feminist $)$ and $P($ bank teller) even if the perceived probability of the feminist conjunct remains much higher than that of bank teller.

A similar situation arises with the Scandinavia scenario (Tentori et al. 2004):

Suppose we choose at random an individual from the Scandinavian population.

Which do you think is the most probable?

- The individual has blond hair.

- The individual has blond hair and blue eyes.

- The individual has blond hair and does not have blue eyes. [e]

[h]

$\left[h_{1} \wedge h_{2}\right]$

$\left[h_{1} \wedge \sim h_{2}\right]$ 
Response/Pothos \& Busemeyer: Can quantum probability provide a new direction for cognitive modeling?

LINDA

SCENARIO

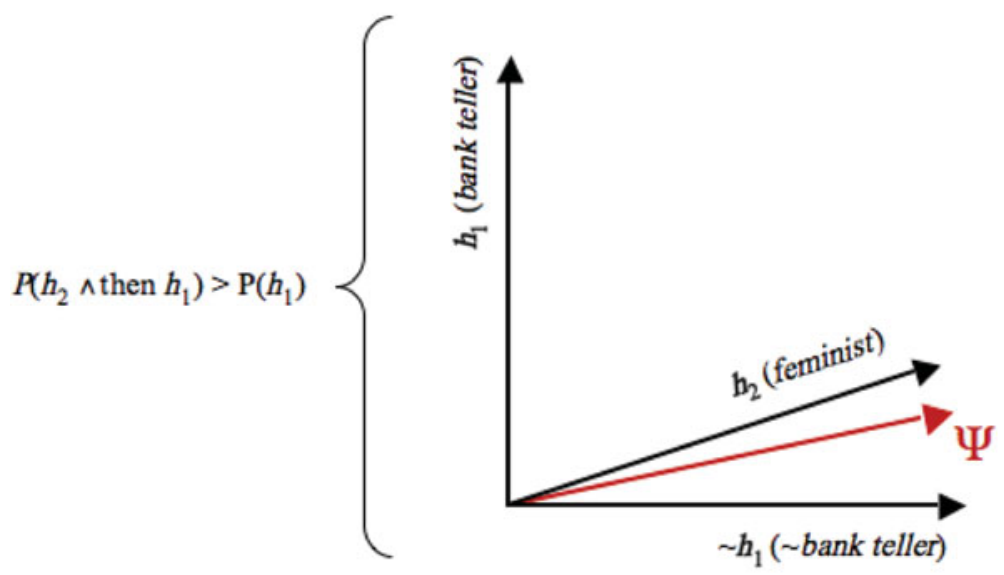

$P\left(h_{2} \wedge\right.$ then $\left.\left.h_{1}\right)<P\left(h_{1}\right)\right\}$

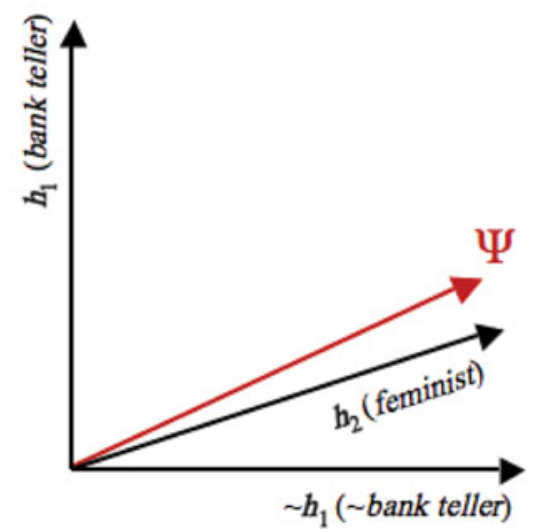

SCANDINAVIA

SCENARIO
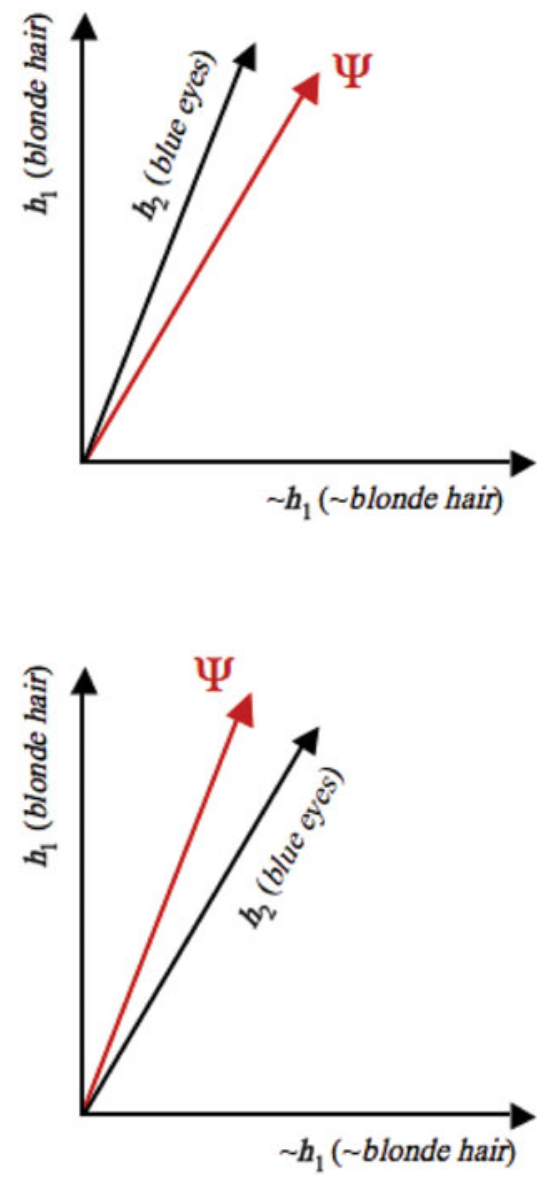

Figure 2 (Tentori \& Crupi). Two different plausible QP representations of the Linda and Scandinavia scenarios. The positioning of the vectors in the top half is compatible with the observed conjunction fallacy judgment $P\left(h_{1} \wedge h_{2}\right)>P\left(h_{1}\right)$, whereas that in the bottom half is not.

Does the QP approach predict the observed prevalence of fallacious choices for $h_{1} \wedge h_{2}$ ? This crucially depends on how we determine the vector space.

The right column of Figure 2 shows two different representations of the Scandinavia scenario (the state vector and the $h_{2}$ basis vector are simply switched). The QP approach allows for both, and both appear reasonable. However, yet again, in the two cases, opposite orderings of $P\left(h_{1} \wedge h_{2}\right)$ and $P\left(h_{1}\right)$ follow from the QP approach. For an observed judgment to be taken as properly supporting the $\mathrm{QP}$ explanation of the $\mathrm{CF}$, the corresponding vector space representation needs to be constrained on independently motivated empirical grounds. Otherwise, we can only say that the QP approach can be made compatible with some (and not all) of the CF data. However, for a putatively comprehensive theoretical framework, being able to accommodate some empirical results does not equal predicting and explaining a phenomenon.

\section{ACKNOWLEDGMENTS}

Research relevant to this work has been supported by the MIUR grant "Problem solving and decision making: Logical, psychological and neuroscientific aspects within criminal justice" (PRIN, n.2010RP5RNM_006) and by Grant CR 409/1-1 from the Deutsche Forshungsgemeinshaft (DFG) as part of the prority program New Frameworks of Rationality (SPP 1516).

\section{Authors' Response}

\section{Quantum principles in psychology: The debate, the evidence, and the future}

\author{
doi:10.1017/S0140525X12003226
}

\section{Emmanuel M. Pothos ${ }^{\mathrm{a}}$ and Jerome R. Busemeyer ${ }^{\mathrm{b}}$}

${ }^{a}$ Department of Psychology, City University London, London EC1V OHB, United Kingdom; ${ }^{\mathrm{b}}$ Department of Psychological and Brain Sciences, Indiana University, Bloomington, IN 47405.

emmanuel.pothos.1@city.ac.uk

jbusemey@indiana.edu

http://www.staff.city.ac.uk/ sbbh932/

http://mypage.iu.edu/ jbusemey/home.html

Abstract: The attempt to employ quantum principles for modeling cognition has enabled the introduction of several new concepts in psychology, such as the uncertainty principle, incompatibility, entanglement, and superposition. For many commentators, this is an exciting opportunity to question existing formal frameworks (notably classical probability theory) and explore what is to be gained by employing these novel conceptual tools. This is not to say that major empirical challenges are not there. For example, 
can we definitely prove the necessity for quantum, as opposed to classical, models? Can the distinction between compatibility and incompatibility inform our understanding of differences between human and nonhuman cognition? Are quantum models less constrained than classical ones? Does incompatibility arise as a limitation, to avoid the requirements from the principle of unicity, or is it an inherent (or essential?) characteristic of intelligent thought? For everyday judgments, do quantum principles allow more accurate prediction than classical ones? Some questions can be confidently addressed within existing quantum models. A definitive resolution of others will have to anticipate further work. What is clear is that the consideration of quantum cognitive models has enabled a new focus on a range of debates about fundamental aspects of cognition.

\section{R1. Beyond classical probability (CP) theory: The potential of quantum theory in psychology}

As we mentioned in our main article, quantum probability theory simply refers to the theory for assigning probabilities to events developed in quantum mechanics, without any of the physics (cf. Aerts, Broekaert, Gabora, \& Sozzo [Aerts et al.]). We are interested in whether the computational properties of quantum models provide a natural way to describe cognitive processes. Quantum cognitive models do not require or assume that any aspect of brain neurophysiology is quantum mechanical. The uniqueness of quantum approaches relative to classical vector models has more to do with their use of projections on subspaces to compute probabilities rather than the fact that complex vector spaces are employed (Behme). Some quantum cognitive models can be realized with only real vector spaces, but others need to use complex vector spaces. The key features of quantum theory arise from certain mathematical theorems, such as Heisenberg's uncertainty principle for incompatible observables, Gleason's theorem (establishing the necessity for computing probabilities from the squared length of projections) and the Kochen-Specker theorem (establishing the necessity for superposition states within a vector space). Because of such theorems, we have properties such as incompatibility and entanglement, which have no analogues in CP theory or classical vector space theory. It is possible to have heuristic versions of such ideas, but a formal implementation with a theory of probability is not possible, unless one employs quantum probability theory. The need for complex vector spaces in particular tends to arise when the empirical result involves violations of the law of total probability, as such violations indicate the presence of interference terms. On a related subject, do quantum models provide a unique insight about cognition? Notions such as incompatibility, superposition, and entanglement have been alien to cognitive psychology (the early relevant insight of William James, which influenced the founding fathers of quantum mechanics, had little impact on psychologists), until quantum cognitive models started to emerge. These are all ideas that have no underpinning in any of the other formal theories.

Lee \& Vanpaemel question whether any insight about cognition can be provided from quantum models. They argue that "quantum theory assumes deterministic causes do not exist, and that only incomplete probabilistic expressions of knowledge are possible," implying that classical theory does allow deterministic causes. We think this distinction is wrong. If I know that Clinton is honest, then there is a probability Probability (Gore|Clinton), that Gore is honest, too. What determines the resolution of my opinion that Gore is honest, given that Clinton is honest? This is as mysterious in probabilistic models of cognition, as in quantum theory. The behavior we observe is probabilistic; therefore, the models must generate predictions that are probabilities. Moreover, a sense of probabilistic determinism can arise in quantum theory in a way analogous to that of classical theory: in quantum theory, if it is likely that thinking that Gore is honest makes Clinton likely to be honest too, then the subspaces for the corresponding outcomes are near to each other. It will not always be the case that a person thinking that Gore is honest will also think that Clinton is honest, but, on average, this will be the case. The corresponding prediction/implication from classical theory would seem identical. Also, the Schrödinger equation (which is used for dynamics in quantum theory) is a deterministic differential equation, analogous to the Kolmogorov forward equation (which is used in stochastic models of decision making). In quantum theory, the only source of non-determinism comes from the reduction of the state vector.

Lee \& Vanpaemel note that "effects of order, context, and the other factors... might be hard to understand, but adopting the quantum approach requires us to stop trying." On the contrary, quantum models provide a formal mathematical framework within which to understand context and order effects in probabilistic judgment. Such effects no longer have to be relegated to ad hoc (e.g., order) parameters or conditionalizations; rather they can be modeled in a principled way, through the relation between the subspaces corresponding to different outcomes. A related point is that "discussion of entanglement...makes it impossible to construct a complete joint distribution between two variables, and therefore impossible to model how they interact with each other." The first part of the sentence is correct, but not the second: Entanglement does not mean that it is impossible to model the interaction between two variables, but rather that it is impossible to do so in a classical way. This is because, for entangled variables, the interaction exceeds classical boundaries. Contrary to what Lee \& Vanpaemel suggest, the objective of quantum cognitive models is exactly to provide insight into those aspects of cognitive process for which classical explanation breaks down.

Gelman \& Betancourt note that, in social science, a theory that allows the complete joint to change after each measurement makes sense, and they outline some situations that might reveal violations of the law of total probability. These observations support the use of quantum theory in corresponding models, although Gelman \& Betancourt also questioned some aspects of current quantum cognitive models.

Pleskac, Kvam, \& Yu (Pleskac et al.) also wondered whether the notion of superposition might reduce insight into quantum models. This is not the case. Note first that a superposition state would have no specific value with respect to a particular question; with respect to another question, the same state may well have specific values. Second, the fact that a superposition state has no specific values (relative to a question) does not mean that this state contains no information. On the contrary, the relation of the state to the various subspaces of interest is the key determining factor in any probabilistic estimate based on 
Response/Pothos \& Busemeyer: Can quantum probability provide a new direction for cognitive modeling?

the state. In other words, a superposition state can still have highly principled, well-defined relations, with respect to all questions that we are considering (that is, all the relevant subspaces in the Hilbert space in which the state is defined).

The debate in the target article focused on the relation between quantum and CP theory, because these are the most comparable quantities. True, we criticize explanations based on individual heuristics. This is because, if one adopts a heuristics approach in cognitive modeling, there are few constraints over what heuristics can be included and the relation between heuristics. Inevitably, this endows a heuristics approach with considerable flexibility. By contrast, with formal probability approaches (classical or quantum), before one writes one line of his or her model, there are plenty of relevant constraints., But, we do not wish to dismiss heuristics (Khalil); rather, we prefer to explore whether heuristics can be interpreted within a more formal framework. Also, we certainly do not dismiss bounded rationality. Indeed, this idea is one of the main ways to motivate a perspective of quantum, as opposed to classical, rationality.

Gonzalez \& Lebiere rightly point out that cognitive architectures, such as Adaptive Character of ThoughtRational (ACT-R), go some way toward addressing our criticisms of approaches based on individual heuristics. This is because a cognitive architecture would have in place several constraints to prevent (some) post hoc modifications. One advantage of cognitive architectures is that their conceptual foundation is usually closer to intuition about psychological process. For example, a cognitive architecture could involve memory and association processes, which are obviously related to human cognition. We agree that cognitive architectures have a high appeal in relation to psychological explanation. One unique advantage of modeling on the basis of formal probability theory is that all the elements of a corresponding model either have to arise from the axioms of the theory (and so are tightly interconnected) or have to correspond to straightforward assumptions in relation to the specification of the modeling problem. Arguably, this is why some of the most famous empirical demonstrations in psychology concern demonstrations that human behavior is inconsistent with the axioms of formal (classical) probability theory (such as Tversky \& Kahneman 1983), whereas corresponding results in relation to cognitive architectures are less prominent. Overall, whereas we agree that models based on cognitive architectures are more appealing than ones based on individual heuristics, we still contend that formal probability theory, classical or quantum, provides a more principled approach for psychological explanation. Note also that, as described in Busemeyer and Bruza (2012), it is possible to embed quantum probability theory into a larger quantum information processing system, using concepts from quantum computing (Nielsen \& Chuang 2000). Converging the merits of formal probability theory with cognitive architectures certainly has a priori explanatory appeal.

\section{R2. Misconceptions on limitations}

Even given the broad description of the theory in the target article, we were impressed that some commentators were able to develop their own variations of quantum models.
This is encouraging in relation to how accessible quantum theory is to a general cognitive psychology audience. Equally inevitably, there were some misperceptions.

At a general level, we assessed quantum models against results that have been at the heart of the decision-making literature for nearly three decades now and have had a profound impact on the development of the debate regarding the application of formal probability theory to cognition. Whether they are simplistic or not, they constitute the first testing ground for any relevant theory (Behme). Also, we do not use "physics as a standard against which to evaluate theories in psychology" (Shanteau \& Weiss). We do not use physics, rather a scheme for assigning probabilities to events from physics. Also, we do not use quantum theory as a standard. Rather, we employ it as an alternative theory, in situations in which it looks as if classical theory failed.

Kaznatcheev \& Shultz (cf. Franceschetti \& Gire) point out that "the quantum probability $(\mathrm{QP})$ approach is a modeling framework: it does not provide guidance in designing experiments or generating testable predictions." Of course, the same can be said of CP theory, neural networks, or mental models. But, does this mean that QP (or any of the other frameworks) do not provide guidance for predictions? We do not think that this is the case. For each modeling framework, a researcher is called to scrutinize the unique features of the framework, and so explore how these unique features can lead to novel predictions. In the case of QP theory, there are properties such as superposition, incompatibility, and entanglement, which offer possibilities for cognitive modeling, which are unique in relation to other frameworks, such as CP theory and neural networks. Relatedly, one cannot just adopt a general formal framework and expect predictions about psychological performance to emerge. One needs to develop specific models (taking advantage of the unique characteristics of the framework) and such models will incorporate assumptions about psychological process that go beyond the prescriptions of the framework. The value of the psychological model is then a function of the relevance of the mathematical theory, as well as the plausibility and motivation of these additional assumptions. An example is our assumption in the quantum model for the conjunction fallacy (and related effects) that more likely predicates are evaluated first. Is this assumption problematic (Kaznatcheev \& Shultz)? All we require is that the decision maker has some mechanism of providing an ordinal ordering of overlap.

A key characteristic of quantum models is that they are not constrained by the unicity principle because they allow for incompatible questions. Perhaps then, QP models can be partly reduced to models of bounded cognition (cf. Rakow)? It is true that we motivate properties of quantum theory, notably incompatibility, partly by appeal to the unrealistic demands from the principle of unicity. In other words, we suggest that the principle of unicity is an unrealistic requirement for cognitive representations, because it is unlikely that we can develop complete joint probability distributions for the information in our environment. This recommends representations that are not constrained by the principle of unicity, that is, incompatible representations. But there are other motivations completely independent of considerations relating to the principle of unicity. For example, processing one question plausibly 
Response/Pothos \& Busemeyer: Can quantum probability provide a new direction for cognitive modeling?

interferes with knowledge about another; the available empirical results strongly indicate this to be the case, at least in some cases (e.g., the conjunction fallacy; Busemeyer et al. 2011). Two independently good reasons for some action appear to interfere with each other (e.g., violations of the sure thing principle in the prisoner's dilemma; Pothos \& Busemeyer 2009). The meaning of individual constituents appears to determine that of a conceptual combination in a way that shows evidence for entanglement (Aerts \& Sozzo 2011b; Bruza et al. 2008). All these lines of evidence could indicate properties of information processing in the cognitive system, plausibly independent of processing limitations, and which are (still) inconsistent with a classical probabilistic description of cognition.

Ross \& Ladyman say that quantum cognitive models "offer no analogous law of time evolution of cognitive states." However, Schrödinger's equation does exactly this. For example, we have assumed that time evolution can correspond to a thought process, whereby the initial state develops to a state, which reflects the outcome of the thought process (e.g., Pothos \& Busemeyer 2009; Trueblood \& Busemeyer 2012; Wang \& Busemeyer, in press). In such quantum cognitive models, the Hamiltonian may not represent the energy of the system, but it does represent its dynamical properties, as is appropriate. Even though there is no corresponding Planck's constant in cognitive models, Planck's constant has a unit-scaling role in physical models. Relatedly, Kaznatcheev \& Shultz question the nature of time development in quantum cognitive models since "given enough deliberation time a participant will always return to a mental state indistinguishable from the one before deliberation." Whereas this is true, deliberation in quantum models typically reflects some sort of ambivalence between, for example, various options in a decision-making problem. Importantly, resolving this deliberation (e.g., accepting an option as a solution to a decision-making problem) typically involves a reduction of the state vector to a basis state. Collapsing a state, which is a superposition state (in relation to possible outcomes for a question or problem), to a corresponding basis state is an irreversible process, which breaks the periodicity in quantum time evolution. This is the assumption we made in all the quantum models just mentioned. Equally, the specific characteristics of quantum evolution in quantum models do not always map well onto cognitive processes, and in some cases classical models appear more successful (Busemeyer et al. 2006).

More specific issues arise in various suggestions from commentators. Newell, van Ravenzwaaij, \& Donkin (Newell et al.) note in their example of multiple projections that "it seems unlikely that the believability of any president should necessarily decrease... as more questions are asked..." We entirely agree, but what these commentators are computing is

$$
\left|P_{\text {LincolnYes }} \ldots P_{\text {GoreYes }} P_{\text {ClintonYes }} \psi\right|^{2}
$$

which is

Prob(Clinton is honest $\wedge$ then Gore is honest $\wedge . .$. then Lincoln is honest)

The conjunction that an increasing number of United States politicians are all honest surely approaches zero and there is no tension with the classical analogue, which would simply be

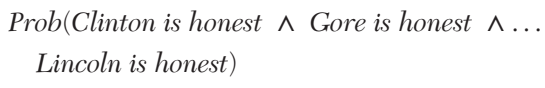

Moreover, if the state vector were to reset itself, we would no longer be computing a conjunction, but rather a conditional probability. For example,

$$
\begin{aligned}
& \text { Prob(Lincoln is honest } \mid \text { Clinton is honest) } \\
& =\left\|P_{\text {Lincoln }} \psi_{\text {Clinton }}\right\|^{2}
\end{aligned}
$$

where $\psi_{\text {Clinton }}$ is a normalized state vector in the Clinton subspace (this is Luder's law). How large this conditional probability is depends on the relation between the Lincoln and the Clinton subspaces. In this particular case, we can probably assume that knowledge that Clinton is honest is uninformative relative to knowledge that Lincoln is honest. Rakow rightly points out that the state knowledge should differ when assessing the premise that Clinton is honest, after deciding that Gore is honest, and vice versa. We completely agree. But there is still tension with CP. Note first that Probability (Clinton honest) * Probability (Gore honest |Clinton honest) is the probability of deciding that Clinton is honest and Gore is honest. Consistently with Rakow, the conditional tells us whether Gore is honest, taking into account the information that Clinton is honest, but, Probability (Clinton honest ${ }^{*}$ Probability (Gore honest $\mid$ Clinton honest $)=$ Probability (Clinton \& Gore). As conjunction is commutative, the classical approach cannot model relevant question order effects, without extra post hoc order parameters.

\section{R3. Empirical and theoretical extensions}

Aerts et al. point out that it is not just QP that is relevant. Rather, there many aspects of quantum theory that are potentially relevant to the modeling of cognition. By QP we do not imply a particular aspect of quantum theory, but rather the entire formalism for how to assign probabilities to events. Having said this, in pursuing the application of quantum theory to cognition, our starting point has been the more basic elements of quantum theory. We agree with Aerts et al. and the other commentators, who pointed out aspects of quantum theory, which could suitably extend existing models.

For example, Shanteau \& Weiss note that happiness (in the target article example) is a continuum, and, therefore, should not be modeled in a binary way. We only use binary values to make the explanations as simple as possible, and it is straightforward to develop models for more continuous responses (see, e.g., Busemeyer et al. 2006, for an application to confidence ratings). For example, our demonstrations could be extended in a way such that the question of interest can have an answer along a continuum, rather than a binary yes-no. Such extensions could then be used to explore differences in performance depending on differences in response format, as Pleskac et al. suggest.

Another issue concerns the output of quantum models. For example, when we evaluate Probability (bank teller) for Linda in the conjunction fallacy problem, what exactly does this 
Response/Pothos \& Busemeyer: Can quantum probability provide a new direction for cognitive modeling?

probability correspond to (Kaznatcheev \& Shultz; Pleskac et al.)? We can use a pure state in a Hilbert space to represent the state of an individual, for example, after a particular experimental manipulation (such as reading Linda's description in Tversky \& Kahneman's 1983, experiment). From this pure state, we can estimate the probability that the individual will assign in relation to, for example, the bank teller property for Linda. We make the reasonable assumption (common to probabilistic models of cognition, whether classical or quantum) that individual response probabilities translate to sample proportions for choosing one option, as opposed to others. Relatedly, it is not clear what is to be gained by using mixed states, in situations in which the emphasis is on individual responses and sample proportions are considered only as indicators of individual behavior (Kaznatcheev \& Shultz).

By contrast, if the emphasis is on ensembles (e.g., how the behavior of a whole group of people changes), then perhaps mixed states are more appropriate (Franceschetti \& Gire). Mixed states combine classical and quantum uncertainties. They concern an ensemble of related elements, for example, particles in a particular physical system or students in an undergraduate class. The representation for each element is a pure (perhaps superposition) state, but we do not really know how many elements are in specific pure states. Therefore, the overall system is described in terms of all the possible pure states, weighted by their classical probability of occurrence. Such mixed states appear particularly useful in situations in which it is impossible to consider a psychological situation in terms of individual elements. For example, mixed states appear appropriate when modeling convergence of belief in a classroom or classification of new instances in a categorization model. Extending quantum models in this way is an exciting direction for future research.

MacLennan raises some interesting points in relation to how the basis vectors corresponding to different questions are determined/learned. Currently, we make the following minimal, but reasonable assumptions. Our knowledge space would be populated with several possible subspaces, corresponding to questions that relate to our world knowledge. For example, we would in general have subspaces corresponding to properties such as being a feminist or a bank teller. Reasonably, the creation of knowledge would involve the creation of new subspaces in our knowledge space. Is there a sense in which the overall dimensionality of the knowledge space would increase, to perhaps reflect an overall quantitative increase in knowledge? Perhaps, and this would require tools from quantum theory, which go beyond those employed in most current models, such as Fock spaces (Aerts et al.). Another issue is how novel information could alter the relation between subspaces. Quantum theory provides a mechanism for doing so, through unitary evolution, although how to specify unitary matrices that can capture the impact of a particular learning experience is beyond current models (but see Ch. 11 in Busemeyer \& Bruza 2012, for some proposals). Such extensions can be a basis for quantum models of learning as well (de Castro), although some corresponding insights are possible from current models also. For example, de Castro discusses the idea that learning can involve a process of forgetting. In current quantum models, after a projection, the initial state loses some of the original information (which is like forgetting), but new insights can be acquired about the initial state (see also Bruza et al. 2009, for the applicability of QP in the understanding of human memory).
Some commentators (Love; Marewski \& Hoffrage) observed that incorporating process assumptions in quantum models could potentially greatly increase the scope and testability of the models. We agree and offer some preliminary responses, which show promise in this direction. First, one distinguishing characteristic between quantum and classical models is that, in the former, for incompatible questions, all operations have to be performed sequentially. This naturally makes computation sequential. In some cases, we have had to make particular assumptions regarding projection order, for example, in the conjunction fallacy. This assumption does not follow from the axiomatic structure of quantum theory, rather it is required because there is no other way to go from the basic assumptions of quantum theory to a mechanism that can be used to predict behavioral results (cf. Kaznatcheev \& Shultz). If one proposes that projection order, as specified at the computational level of a quantum model, maps onto a psychological time parameter for the actual computations at the algorithmic level, then projection order could be tested as a process aspect of quantum models. Second, in CP models in decision making (such as random walk/diffusion models) it is common to employ a time development process (from the forward Kolmogorov equation), but in this case, the constraints at baseline (e.g., the law of total probability) would also apply at any subsequent time. However, in quantum models, time evolution (from Schroedinger's equation) can be quite different. The states at baseline may obey the law of total probability, but after time evolution the law of total probability may break down. We have fruitfully employed this approach in a variety of situations (Pothos \& Busemeyer 2009; Trueblood \& Busemeyer 2012; Wang \& Busemeyer, in press). In such quantum models, the time development of the initial state into another one (for which the law of total probability can break down) is necessarily interpreted as a process of deliberation of the relevant evidence. Therefore, such models do have a process component, although a lot more work needs to be done along these lines. Finally, Busemeyer et al. (2006) explored a random walk quantum model for a decision-making task. Therefore, there is some groundwork on how to employ quantum principles to implement process models proper.

Behme and Corr highlighted the issue of how quantum cognitive models scale up to more realistic empirical situations. It is probably fair to say that both the application of classical theory and quantum theory have been focused on idealized, laboratory situations, because of the complexity of taking into account a full set of realistic constraints in a principled way. An example of progress in this respect is the model for behavior in the Prisoner's Dilemma games of Pothos and Busemeyer (2009) (see also Trueblood \& Busemeyer 2012; Wang \& Busemeyer, in press). In both the classical and the quantum versions of this model, it was possible to incorporate the idea of cognitive dissonance (Festinger 1957), so that beliefs and actions would converge to each other. The weight of this process, in relation to evaluating payoff, could not be predicted a priori, and, therefore, was set with a free parameter. Therefore, "more realistic" mechanisms can, in principle, be implemented in both classical and quantum models. There is still a question of whether these mechanisms allow successful coverage of empirical data. One point is 
Response/Pothos \& Busemeyer: Can quantum probability provide a new direction for cognitive modeling?

that for empirical data that violate the basic axioms of classical theory, even postulating additional realistic mechanisms (such as cognitive dissonance), is still insufficient.

Specifically in the area of preference formation, Kusev \& van Schaik suggest that preferences are often constructed in an ad hoc, content-dependent way and Corr also highlights the potential impact of mood or other emotional variables on preference formation for the same option. At face value, incompatibility in quantum theory can allow evaluation of probabilities or preferences in a perspective-dependent way. Corr also points out that introducing considerations relating to mood or emotion may lead to "nonlinear dynamical effects." Because probability in quantum models is assessed by squaring the length of the state vector, there is more potential for capturing any corresponding non-linearities in the dynamics of a psychological system, although clearly, whether this is actually the case or not can only be assessed in the context of specific models. The work of Pothos and Busemeyer (2009), Trueblood and Busemeyer (2012), and Wang and Busemeyer (in press) takes advantage of non-linearities in the development of probabilities for particular events, to capture results that are puzzling from a classical perspective.

A more general point regarding the scalability of quantum versus classical models concerns the requirements from the principle of unicity for the latter. Classically, there is the demanding requirement that there is a complete joint probability distribution for the outcomes of all questions. This requirement means that every time an additional question is included, the complete joint needs to be augmented to take into account the event and, also, all corresponding marginal probability distributions need be specified as well. Therefore, additional questions greatly increase the complexity of the required representation. By contrast, representations of incompatible questions in a quantum model can coexist in a low dimensionality space. The introduction of further incompatible questions does not necessarily increase the dimensionality of the space and the subspaces for different questions can be related to each other through a unitary operation. Overall, representation of additional questions (for a hypothetical system) in a quantum model implies lower representational demands than in a classical one.

A promising new domain of application of quantum theory is learning and memory (as discussed, see de Castro; Bruza et al. 2009). Baldo also discusses how the main elements of signal detection theory can be represented in a Hilbert space. This is an interesting extension to current work with quantum cognitive models. One advantage is that this provides an intuitive visual representation of some related visual phenomena. An issue for future research would be whether there are empirical findings in signal detection theory, which necessitate a Hilbert space representation, for example, in relation to violations of the law of total probability or order/context effects. In the more general area of perception, there has been some work along such lines, by Conte et al. (2009) and Atmanspacher and Filk (2010).

\section{R4. Empirical challenges}

Whether researchers accept the quantum framework as a viable alternative to $\mathrm{CP}$ theory is partly an empirical issue. We review some important empirical challenges, as highlighted by the commentators.

Hampton points out the quantum model for the conjunction fallacy cannot be applied to understand the guppy effect-a situation in which the conjunction is more probable than both the individual constituents. Note first that we agree that researchers should be looking to understand similarity and decision-making processes within the same modeling framework (Shafir et al. 1990). However, the mechanics of projection in the Linda problem are quite different to those for the guppy effect. In the Linda problem, the initial state reasonably corresponds to the impression participants form after the Linda story. Then, this initial state is assessed against either the single property of bank teller or the conjunction of bank teller and feminist properties. By contrast, in the guppy problem, the initial state is a guppy, a pet fish. If we were to adopt a representation analogous to the one for the Linda problem, we would need an initial state that exists within a subspace for pet fish, which corresponds to the concept of guppy. Then, a guppy has maximum alignment with the pet fish subspace (probability = 1) and less alignment with either the pet or the fish subspaces. In other words, in the guppy case it makes no sense to consider the conjunction of pet and fish properties for guppy (as in the Linda case), because by definition a guppy is constructed to be a pet fish; the pet fish characterization for guppy it tautological, rather than a conjunction. By contrast, Linda is not by definition a bank teller and a feminist, rather Linda corresponds to a particular state vector, related to all these properties. Therefore, the guppy effect can be reproduced with the quantum model for the Linda problem, albeit in a trivial way. A problem with Hampton's model is that, as with averaging models generally, it fails to account for violations of independence, that is, findings such that $\mathrm{A} 1+\mathrm{B} 1>\mathrm{A} 2+\mathrm{B} 1$, but $\mathrm{A} 1+\mathrm{B} 2<\mathrm{A} 2+\mathrm{B} 2$ (Miyamoto et al. 1995). Finally, an alternative, not trivial, quantum model for the guppy effect has been proposed by Aerts (2009; Aerts \& Gabora 2005). This model is different in structure from the Linda model (for example, it relies on Fock spaces, which generalize Hilbert spaces in a certain way), nevertheless both models are based on the same fundamental quantum principles.

Tentori \& Crupi discuss an interesting finding according to which, for a Russian woman, Probability (NY and I) is higher than Probability (NY and $\sim$ I), whereby NY means living in New York and I being an interpreter. They show how an application of the quantum model for the conjunction fallacy in this case appears to break down. However, their demonstration is based on a two-dimensional representation; but quantum models are not constrained to two dimensions. The dimensionality of the representation will be determined by the complexity of the relation between the corresponding questions. Dimensionality is not a free parameter: we just need to ask whether all the information in a problem can have an adequate representation in, for example, two versus three dimensions. Note that if one parameterizes the angles between subspaces, then dimensionality can increase the number of parameters. However, with most current demonstrations, the focus has been on showing whether a result impossible to predict with (typically) CP principles can emerge from a quantum model.

Regarding the problem of the Russian interpreter living in New York, the fact that a two-dimensional representation is 
Response/Pothos \& Busemeyer: Can quantum probability provide a new direction for cognitive modeling?

inadequate can be seen by noting that the state vector, assumed to be within the Russian subspace, has to be very close to both the $\sim \mathrm{I}$ and $\sim \mathrm{NY}$ rays, as, clearly, both of these are extremely unlikely properties for a Russian woman. Moreover, the I and NY rays cannot be close to each other, because knowing that a person is an I does not really tell us very much about whether he/she lives in New York (but equally it does not preclude that the person is living in New York). One can satisfy these tricky constraints (along with the simpler one that the state vector has to be closer to the $\sim$ NY ray than the $\sim$ I one) in a three-dimensional space. Note, finally, that the quantum model for the conjunction fallacy assumes that the more likely predicate is evaluated first. Then, it emerges that Prob (I and then NY) $>\operatorname{Prob}(\sim \mathrm{I}$ and then $\mathrm{NY}$ ), as required. Figure R1A-C illustrates the representation, but it is also possible to verify the prediction directly. For example, setting $\mathrm{NY}=[0.39,0.92,0.047], \sim \mathrm{NY}=[0.087$, $-0.087,0.99], \quad \mathrm{I}=[-0.39, \quad 0.92,0.047], \quad \sim \mathrm{I}=[-0.087$, $-0.087,0.99]$, Russian $=[0.029,0.087,0.99]$, we have that Prob $(\mathrm{I}$ and then NY $)=0.0065$ and Prob $(\sim \mathrm{I}$ and then NY $)$ $=0.0043$. We note that this is a toy demonstration, which is meant to neither reproduce specific numerical values nor cover all the relevant constraints of Tentori \& Crupi's example (for these, a more general model would be needed). Nevertheless, the demonstration does capture their main result.

A key question is exactly how definitive is the evidence we consider in favor of quantum theory. Kaznatcheev \& Shultz rightly point out that there are rarely clear-cut answers. They discuss the case of Aerts and Sozzo's (2011b) demonstration of entanglement and conclude that one cannot necessarily infer the need for quantum theory, over and above classical theory. Likewise, violations of the law of total probability are amenable to classical explanations, for example, with appropriate conditionalizations on either side of a law of total probability decomposition. We agree that an empirically observed violation of the law of total probability does not definitely prove the inapplicability of CP. However, classical explanations would rely on post hoc parameters or conditionalizations, with unclear explanatory value. In quantum models, because of the order and context dependence of probabilistic assessment, it is often the case that corresponding empirical results can emerge naturally, (nearly) just from a specification of the information in the problem (as in (a)

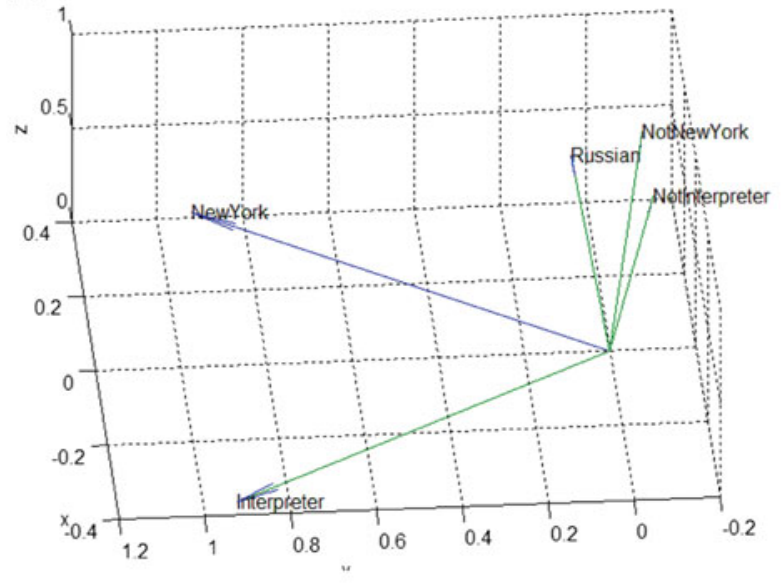

(b)

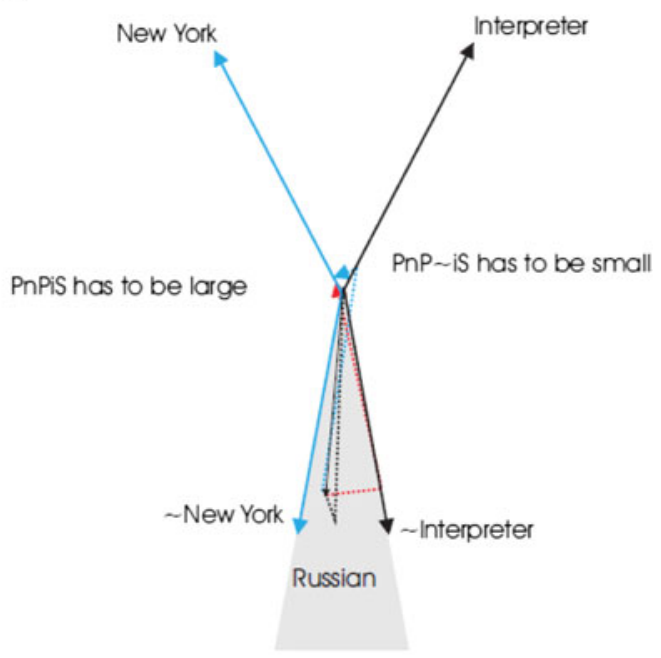

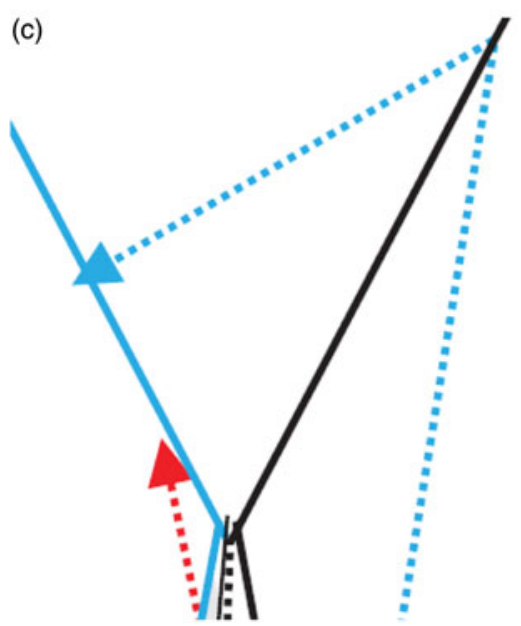

Figure R1. A representation that can account for the main finding discussed by Tentori \& Crupi, that a Russian woman is more likely to be living in New York and be an interpreter, than be living in New and not be an interpreter. Part C shows a magnification of the central part of $\mathrm{B}$. 
Response/Pothos \& Busemeyer: Can quantum probability provide a new direction for cognitive modeling?

the model for the conjunction fallacy; Busemeyer et al. 2011). Relatedly, Shanteau \& Weiss questioned the complexity of quantum cognitive models. On the contrary, for the reasons just discussed, it seems clear that quantum models for empirical results such as the conjunction fallacy are actually simpler than matched classical ones.

Quantum models can involve both incompatible and compatible questions, classical models only the latter. Are quantum models more flexible than classical ones? Do quantum models subsume classical ones (Houston \& Wiesner, Kaznatcheev \& Shultz)? There are three responses. First, in all the quantum models that have been employed so far, quantum transition (unitary) matrices need to obey double stochasticity, a constraint not applicable to classical transition matrices. Second, as Atmanspacher notes (cf. Atmanspacher \& Roemer 2012), it is possible to test in a very general way whether a quantum approach can apply to a given situation. Consider a particular question and the conjunction of this question with another one, assumed to be incompatible. If the individual question fails to be compatible with the conjunction (i.e., there are order effects between the question and the conjunction), then a Hilbert space representation is precluded. Third, there is the law of reciprocity, according to which the probability of transiting from one pure state to another must equal the probability of transition in the opposite direction. In other words, if we imagine a state vector in a unidimensional subspace $\mathrm{A}, \psi_{\mathrm{A}}$, and one in a unidimensional subspace $\mathrm{B}, \psi_{\mathrm{B}}$, then

$$
\left\|\mathrm{P}_{\mathrm{A}} \psi_{\mathrm{B}}\right\|^{2}=\left\|\mathrm{P}_{\mathrm{B}} \psi_{\mathrm{A}}\right\|^{2}
$$

which means that

$$
\operatorname{Probability}(\mathrm{A} \mid \mathrm{B})=\operatorname{Probability}(\mathrm{B} \mid \mathrm{A})
$$

but in classical theory,

$$
\operatorname{Probability}(\mathrm{A} \mid \mathrm{B}) \neq \operatorname{Probability}(\mathrm{B} \mid \mathrm{A})
$$

in general. Wang and Busemeyer (in press) have tested the law of reciprocity in question order effects and found it to be upheld with surprising accuracy. It is important to note, however, that the prediction

$$
\operatorname{Probability}(\mathrm{A} \mid \mathrm{B})=\operatorname{Probability}(\mathrm{B} \mid \mathrm{A})
$$

only holds for rays and not for events described by higher dimensional subspaces.

These are abstract arguments and do not necessarily bear on the issue of the relative complexity between particular models. Are particular quantum models more flexible than matched classical ones? This is an empirical issue. So far, there has been one corresponding examination, by Busemeyer et al. (2012), as reviewed in the target article. Even taking into account relative model complexity, a Bayesian analysis still favored the quantum model over a matched classical one. This is one examination for one particular quantum model, but this examination does provide the only available evidence and this evidence does its small bit toward undermining a claim that quantum models are in general more flexible than matched classical ones.
Rakow notes that perhaps a major source of flexibility of quantum models arises from a choice of whether some questions are treated as compatible or incompatible (see also Navarro \& Fuss; Newell et al.). This point is overstated. First, the default assumption is that questions about distinct properties are incompatible. Noori \& Spanagel provide a good expression of the relevant idea: "Incompatibility between questions refers to the inability of a cognitive agent in formulating single thoughts for combinations of corresponding outcomes." Conversely, compatible properties are such that it is possible to combine them in a single corresponding thought. It is usually possible to empirically determine incompatibility between two questions, as conjunction is usually subject to order effects. In the context of Gore, Clinton question order effects, Khalil argues that the questions "involve symmetrical and independent information. Information with regard to each question can be evaluated without the appeal to the other" to conclude that these questions cannot be incompatible. All these reasons are exactly why it is so puzzling that empirically we do observe order effects in the Clinton, Gore question. Crucially, the considerations Khalil provides are not really the ones that are relevant in determining incompatibility; rather, these would relate to the presence of order effects.

Second, once incompatibility/compatibility is resolved, a QP model is limited in terms of both the relation between the various subspaces and the location of the state vector. These relations can be often determined in a principled way, via correlations between answers to relevant questions and considering the participants' frame of mind in the study, and do not prejudge the accuracy of model predictions. For example, Tentori \& Crupi note that variations in the representation for the quantum model for the conjunction fallacy can provide predictions inconsistent with empirical results. Our representation for this model (Fig. 2 in the target article) was created to be consistent with three constraints: Probability ( bank teller) is as high as possible; Probability (feminist) is as high as possible; finally, the ray for the feminist question is relatively neutral with respect to the bank teller, not bank teller questions, but slightly closer to the not bank teller question. All these three constraints are part of the original specification of the problem. They are self-evident and there is no circularity or subtlety in creating a quantum representation for the Linda problem that is consistent with these constraints. Even though there is some flexibility in the exact angles, any plausible quantum representation would need to be consistent with these constraints. Tentori \& Crupi's variation in the bottom left hand side of their Figure 2 violates the first constraint, and, therefore, their corresponding conclusion is incorrect.

Exactly how precise the specification of subspaces and the state vector is will depend on exactly how precise we require the predictions to be. For example, in the conjunction fallacy problem, if we were interested in predicting a specific probability for preferring the conjunction to the single predicate statement, we could fit the angle between the state vector and one of the subspaces, but this is less interesting, because it is a problem of fitting a one parameter problem to a one degree of freedom result. What we believe is much more interesting is whether a range of sensible placements for the bank teller, feminist subspaces and the state vector can lead to a conjunction fallacy. 
Response/Pothos \& Busemeyer: Can quantum probability provide a new direction for cognitive modeling?

The Linda fallacy is an excellent example with which to demonstrate the quantum approach to decision making, because the state vector and location of subspaces are well constrained. By contrast, in the Scandinavia scenario discussed by Tentori \& Crupi, there are fewer such constraints. We agree with Tentori \& Crupi that a particular configuration can lead to a conjunction fallacy, but others do not. The crucial point is that QP theory in general and the quantum decision-making model of Busemeyer et al. (2011) in particular can naturally produce conjunction fallacies. Furthermore there are numerous testable consequences that follow from this model that are independent of parameters and dimensionality. By contrast, no CP model can naturally produce a conjunction fallacy, because a fundamental constraint in such models is $\operatorname{Prob}(\mathrm{A}) \geq \operatorname{Prob}(\mathrm{A} \wedge \mathrm{B})$ regardless of what events $\mathrm{A}$, B are employed. To follow from Tentori \& Crupi's related criticism, the predictions from quantum models are well constrained, to the extent that the corresponding empirical problem is well specified as well.

Because quantum theory is a formal theory of probability, all the elements in quantum theory constrain each other. As Stewart \& Eliasmith note, it is possible to create neurally plausible vector-based representations, in which probability for the outcome of a question can depend on a variety of factors, for example, the length of projections from all competing vectors. Such schemes perhaps motivate a vector-based approach to representation, without the constraints from Gleason's theorem. However, without Gleason's theorem, there appears to be no principled reason to choose among the various possibilities for how to generate decisions. Gleason's theorem exactly justifies employing one particular scheme for relating projection to decision outcome and, moreover, allows projection to be interpreted in a highly rigorous framework for probabilistic inference. Employing Gleason's theorem reduces arbitrary architectural flexibility in vector models.

The debate over which empirical tests can reveal a necessity for a quantum approach, as opposed to a classical one, will likely go on for a while. Dzhafarov \& Kujala discuss the idea of the selectiveness of influences in psychology, and draw parallels with empirical situations in physics that can lead to violations of Bell's or the CHSH (Clauser-Horne-Shimony-Holt; e.g., Clauser \& Horne 1974) inequalities. In physics, the latter has been taken as evidence that superposition states exist and, therefore, as support for a view of physical reality with quantum mechanics. Can analogous tests be employed, in relation to selective influences or otherwise, to argue for the relevance of quantum theory in psychology, and how conclusive would such tests be? Some theorists (Aerts \& Sozzo 2011b; Bruza et al. 2008) have explored situations that show the entanglement of cognitive entities, for example, the meanings of constituent words in a conceptual combinations. Others (Atmanspacher \& Filk 2010) have considered entanglement in perception. The focus of our work has been on decision-making results, which reveal a consistency with quantum principles (e.g., incompatibility, interference) and away from classical ones, but in psychology, and unlike in physics, there are challenges in any empirical demonstration. A key problem is that the psychological quantities of relevance are not always as sharply defined as physical quantities (e.g., word meaning vs. momentum). Another problem is that in physical demonstrations it is often possible to eliminate noise to a very high degree, but this is much less so in psychological experiments.

Relatedly, MacLennan points out that, if we know the relation between two incompatible subspaces (as we must, if we are to build a well-specified quantum model), then it is possible to quantify the lower limit of the product of the uncertainties for the corresponding observables. This raises the possibility that lowering one uncertainty may lead to increases in the other, and vice versa. This is a novel and exciting possibility, not only in terms of novel predictions, but also in terms of, perhaps, tests for whether two properties are incompatible or not.

Blutner \& beim Graben are more ambitious, and ask whether it can be demonstrated that, in some case, "quantum probabilities are such a (virtual) conceptual necessity." Are there general properties of a system of interest (an aspect of cognition), which reveal a necessity for quantum probabilities? This is surely a very provocative thesis! These commentators put forward the idea of quantum dynamic frames. The main idea in quantum dynamic frames is that a measurement (typically) changes the system. When there is a system of this type, under general conditions, quantum dynamic frames necessarily lead to the structures for probabilistic assessment, as in standard quantum theory. Therefore, the question of whether QP theory is relevant in cognitive modeling or not can be partly replaced with the more testable/specific question of whether measurement can change a cognitive state.

\section{R5. Neural basis}

It is possible to utilize quantum theory to build models of neural activity. MacLennan suggests that the distribution of neural activity across a region of cortex could be described by a quantum wave function. Whereas this possibility is intriguing, one has to ask whether there is any evidence that such aspects of brain neurophysiology involve quantum effects or not. Banerjee \& Horwitz offer a preliminary perspective in this respect. They note that to study "auditory-visual integration one would design unimodal control tasks (factors) employing visual and auditory stimuli separately, and examine the change of brain responses during presentation of combined visual-auditory stimuli." This is effectively a factor-based approach, but one which, in a classical analytical framework, does not take into account potential interference effects. Rather, the standard assumption is such that the brain regions engaged by each unimodal task have an additive impact in relation to the multimodal task. Relatedly, Banerjee \& Horwitz point out that, when considering functional networks, it is often the case that any "any alteration in even a single link results in alterations throughout the network." This raises the possibility that the function of nodes in a functional network cannot be described by a joint probability function, so that states of the network may exhibit entanglement-like effects. Clearly, we think that such applications are very exciting (cf. Bruza et al. 2009).

The issue of quantum neural processing, as described previously, is distinct to that of the neural implementation of quantum cognitive models. According to Mender, if a quantum approach to cognition was tied to a neural/physical theory of implementation, then, perhaps, pairs of 
Response/Pothos \& Busemeyer: Can quantum probability provide a new direction for cognitive modeling?

incompatible observables could be extracted from such a theory directly. By contrast, in our current, mostly topdown, approach we have to infer incompatible observables in purely empirical ways. Moreover, in such a cognitive approach it is not really possible to specify an analogue to Planck's constant. How much does the quantum cognition program suffer from the lack of a clear theory of neural implementation? We believe not very much. We seek to infer the computational (and possibly process) (Love; Marewski \& Hoffrage) principles of cognitive processing. Such principles would be consistent with a variety of implementational approaches at the neural level. Although ultimately, cognitive computation and neural implementation have to constrain each other, these constraints are often fairly loose and in actual practice do not typically impact on the specification of computational models. Mender is correct that, perhaps, neural implementation considerations might help us with the determination of pairs of incompatible properties, but even just focusing on the fact that certain pairs of observables are incompatible, and information about their relation (which can be inferred from general properties of the observables), seems to provide us with considerable explanatory power.

There is a deeper issue here of whether there is something about the computations involved in quantum cognitive models that precludes neural instantiation. Noori \& Spanagel argue that brain neurobiology is deterministic and, moreover, that it deterministically specifies behavior. Therefore, how can we reconcile neural/biological determinism with behavioral stochasticity? First, the work reviewed in Noori \& Spanagel's commentary shows a causal relationship between neurochemical changes in the brain and behavior, which is, perhaps, unsurprising, but the further step of assuming complete determinism between brain neurochemical reactions and behavior is unwarranted. Such a step would necessitate assumptions that human behavior is completely predictable from the current brain state. Given the number of neurons involved, determinism in such a sense, even if "real," would be impossible to prove or disprove. Second, we do not really know what is the neural reality of stochasticity in behavioral probabilistic models (quantum or classical). At the neuronal level, it may be the case that such stochasticity is resolved in a deterministic way. Interestingly, Shanteau \& Weiss criticize quantum models in exactly the opposite way, that is, by arguing that they are too deterministic for modeling unpredictable human behavior. We are unconvinced by such arguments. Whichever perspective one adopts in relation to the determinism of brain neurophysiology, at the behavioral level there is an obvious need to represent uncertainty in human life. Therefore, at that level, it makes sense to adopt modeling frameworks that formalize uncertainty (whether classical or quantum).

A related issue is whether quantum cognitive models require quantum processes at the neuronal level (and, therefore, a quantum brain). This does not appear to be the case. Two commentaries (Atmanspacher; Blutner \& beim Graben) discuss how incompatibility, a key, if not the fundamental, property of quantum cognitive models, can arise in a classical way. This can happen if measurement is coarse enough that epistemically equivalent states cannot be distinguished. Hameroff, however, strongly argues in favor of a quantum brain as the source of quantum behavior (as he says, if it walks and thinks like a duck then it is a duck). In the past, his work has mainly addressed the problem of consciousness. But in this comment, he advances his ideas by providing a detailed discussion for how his orchestrated objective reduction (Orch OR) model can be extended to incorporate subspaces and projections for complex thoughts, as would be required in quantum cognitive models. This is a detailed proposal for how quantum computations can be directly implemented in a way that the underlying neuronal processes are quantum mechanical. Such ideas are intriguing, and he mentions a growing literature on quantum biology, but currently this direction remains very controversial.

Finally, Stewart \& Eliasmith note how the neural engineering framework can be employed in relation to vector representations and operations. An important point is that the problem of neural implementation of quantum models is not just one of representing vectors and standard vector operations, such as dot products. One also needs to consider how characteristics uniquely relevant to QP theory could be implemented, notably superposition and incompatibility. It is these latter properties that present the greatest challenge. In other words, for example, one would need to consider what in the nature of neural representation gives rise to incompatibility between different properties (as opposed to merely implementing subspaces with basis vectors at oblique angles). Stewart \& Eliasmith also note that allowing tensor product operations in a behavioral model raises questions in relation to how an increasing number of dimensions are represented, but, for example, could one not envisage that there are different sets of neurons to represent the information in relation to the Happy and Employed questions? Then, when a joint representation is created, the two sets of neurons are simply brought together. It is also worth noting that Smolensky et al. (in press) have argued against the perception that increases in dimensionality caused by tensor products are problematic. It is currently unclear whether such dimensionality increases present a problem in relation to neural implementation.

\section{R6. Rationality}

The question of whether an account of human rationality (or not) should emerge from quantum cognitive models partly relates to their intended explanatory level. Navarro \& Fuss argue that quantum theory does not make sense as a normative account of everyday inference. Instead, their view is that CP theory is the better approach for computational level analysis, as "it is the right tool for the job of defining normative inferences in everyday data analysis." However, the latter statement is a belief, not an empirically established truth. Many complex systems are sensitive to measurement, and a difficulty arises for classic probability theory when measurement changes the system being measured. Suppose we obtain measurements of a variable $\mathrm{B}$ under two different conditions: one in which we measure only $\mathrm{B}$ and another in which we measure A before $\mathrm{B}$. If the prior measurement of $\mathrm{A}$ affects the system, then we need to construct two different CP models for $\mathrm{B}$, one when $\mathrm{A}$ is measured and one when $\mathrm{A}$ is not measured. Unfortunately, these two CP distributions are stochastically unrelated, which means that the two 
Response/Pothos \& Busemeyer: Can quantum probability provide a new direction for cognitive modeling?

distributions have no meaningful relation between each other (cf. Dzhafarov \& Kujala) and a more general theory is required that can construct both distributions from a single set of elements. This is what quantum probability theory is designed to do, although as Gelman \& Betancourt point out, there may be other ways to achieve this generalization.

A related point is that, if for the moment one does away with normative considerations, both CP and QP theory have analogous structure and objectives with respect to cognitive explanation. Both theories make sense as topdown (axiomatic) theories: in neither case are there assumptions, for example, that the cognitive system represents uncertainty in particular ways. Rather, the claim is that behavior, however it is produced, is consistent with the constraints of CP (or QP) theory. Therefore, we think that a characterization as "top-down" is a more accurate way to label both CP and QP explanations, without reference to normative considerations. As noted in response to other commentators (Love; Marewski \& Hoffrage), we aspire to extend quantum models in a way such that process assumptions are incorporated, and we think that quantum models offer promise in this respect, but such extensions start from the "explanatory top."

Oaksford expresses several intuitions regarding the rational status of classical probability theory. For example, he notes that "If one follows the laws of probability in the Kolmogorov axioms, one will never make a bet one is bound to lose, and therefore it can provide a rational standard," but as we argued in the target article, an appeal to the Dutch book theorem cannot be used to motivate the rational status of CP theory, as this is only valid with the psychologically unrealistic assumption of utility as value maximization. He also notes that "If one follows the laws of logic, one will never fall into contradiction, and, therefore, logic can provide a rational standard," but this argument is undermined by Goedel's incompleteness theorems, because for many axiomatic systems either not all conclusions can be axiomatically derived or, if they can, they will be inconsistent.

Oaksford also observes that classical normative theory is based on clear intuition. For example, according to standard logic and CP theory, it is irrational to believe both $\mathrm{p}$ and not-p are true. If two events, A versus B, are mutually exclusive, then this is true for QP theory as well and the two events have to be compatible in the quantum sense. Also, the same event B cannot be both true and not true simultaneously. The subtle issue arises when events A versus B are incompatible, because in this case, knowing $\mathrm{A}$ is true prevents us from concluding that $\mathrm{B}$ is true or $\mathrm{B}$ is not true. If $\mathrm{A}$ is incompatible with $\mathrm{B}$, then we cannot discuss their truth values at the same time. Suppose that we model the truth and falsity of a logical statement as incompatible events, although clearly related in a specific way (one could employ suitably opposite probability distributions). Then, the events "statement A is true" and "statement A is false" no longer correspond to orthogonal subspaces, and, therefore, it is no longer the case that Prob(statement A is true and then statement A is false) has to be 0 . By contrast, Blutner et al. (in press) showed that the same approach could never produce Prob (statement $\mathrm{A}$ is true and statement $\mathrm{A}$ is false) $\neq 0$ in a corresponding CP model. This is an important point, because empirically naïve observers do sometimes assign non-zero probability values to such statements. In a now wellknown demonstration, Alxatib and Pelletier (2011) showed that, in the case of describing a person as tall or not, borderline cases admit the characterization 'tall and not tall' (other predicates and examples were used). Vague instances with respect to a property $\mathrm{p}$ can often be described as "p and not p" (Blutner et al. in press).

Grace \& Kemp observe that the behavior of nonhumans is sometimes more consistent with classical optimality, than is that of humans. For example, they note that nonhumans (e.g., pigeons) often avoid the base rate fallacy. Houston \& Wiesner also suggest that apparent violations of classical optimality in nonhumans can sometimes be explained if one revises assumptions about the environment. Does the debate between classical, quantum rationality apply to humans as well as nonhumans? The nonhuman studies typically involve extensive training, and this may provide the opportunity to form a compatible representation of the relevant events (by experiencing their joint occurrences). Even with humans, the base rate fallacy can be greatly reduced, when knowledge about the events is derived directly from experience. Another possibility is that perhaps the ability to reason with incompatible representations requires a greater degree of cognitive flexibility (lacking in nonhumans?), so that the same outcome can be approached from different perspectives.

A related perspective is offered by Ross \& Ladyman, who suggest that Bayesian reasoning may work well in small worlds, but not in large worlds. We agree that this seems plausible, in that there would be limited sets of questions that can be represented in a compatible way (and that, therefore, CP theory can apply), but these sets would be incompatible with other sets, so that when considering the totality of questions, the requirement from the principle of unicity need not be fulfilled, and a non-classical picture emerges.

Oaksford is concerned that we do not provide a view of human rationality, according to QP theory. We suggest a notion of human rationality in terms of optimality that arises from formal, probabilistic inference. What marks a rational agent is the consistency that arises from assessing uncertainty in the world in a lawful, principled way. Such a minimalist approach to rationality can be justified partly by noting that traditional arguments for Bayesian rationality, such as long-term convergence and the Dutch book theorem, break down under scrutiny. If one accepts this minimalist approach to rationality, then the question becomes, which formal system is most suitable for human reasoning? Oaksford and Chater (2009) themselves convincingly argued against classical logic, because its property of monotonicity make it unrealistically stringent in relation to the ever-changing circumstances of everyday life. In an analogous way, we argue against CP theory, in relation to the unicity principle. But can we reconcile the perspective dependence of probabilistic assessment in quantum theory with the need for predictive accuracy in human decision making? In its domain of original application (cf. Oaksford; quantum theory was not created to apply to the macroscopic physical world), in the microscopic world, quantum prediction can be extremely accurate, despite all this (seeming) arbitrariness, which arises from perspective dependence. Our point is that perspective dependence does not reduce accuracy. Is the mental world like the microscopic physical world, for which quantum theory was originally developed? To the extent that psychological 
References/Pothos \& Busemeyer: Can quantum probability provide a new direction for cognitive modeling?

behavior exhibits properties such as incompatibility, interference, and entanglement, we believe that the answer is yes.

Lee \& Vanpaemel present another objection to quantum theory. They note the extent to which a limited number of physicists have objections to quantum theory. They provide a telling quote from Jaynes, in which he strongly questions the value of quantum theory in physics. (However, we recommend reading Bub [1999] rather than Jaynes, for a more comprehensive interpretation of quantum theory.) To clarify this issue, physicists do not object to the formal (mathematical) form of quantum theory. They debate its interpretation. Our applications to cognition have used the mathematics, and we have avoided taking any stand on the interpretation of quantum theory. Leaving aside the fact that no other physical theory has had such a profound impact in changing our lives (e.g., through the development of the semiconductor and the laser), few if any physicists think that quantum theory is going into retirement soon. For completeness, it is worth noting that Aspect's work famously and definitively supported quantum theory against Einstein's classical interpretation of Bell's hypothetical experiment (e.g., Aspect et al. 1981). Any introductory quantum mechanics text will outline the main ideas (e.g., see Isham 1989). Quantum theory is a formal theory of probability: it remains one of the most successful in physics and we wish to explore its possible utility in other areas of human endeavor.

In conclusion, the wide variety of thought-provoking comments, ranging across criticisms to empirical challenges to debates about fundamental aspects of cognition, attest to Sloman's view that "quantum theory captures deep insights about the workings of the mind" (this is part of his review for Busemeyer \& Bruza's 2012 book, Busemeyer \& Bruza 2012).

\section{References}

[The letters "a" and " $r$ " before author's initials stand for target article and response references, respectively]

Aerts, D. (1986) A possible explanation for the probabilities of quantum mechanics. Journal of Mathematical Physics 27:202-10. [DA]

Aerts, D. (2007) General quantum modeling of combining concepts: A quantum field model in Fock space. Available at: http://uk.arxiv.org/abs/0705.1740. [DA]

Aerts, D. (2009) Quantum structure in cognition. Journal of Mathematical Psychology 53:314-48. [DA, arEMP]

Aerts, D. (2011) Quantum interference and superposition in cognition: Development of a theory for the disjunction of concepts. In: Worldviews, science and us Bridging knowledge and its implications for our perspectives of the world, ed. D. Aerts, J. Broekaert, B. D’Hooghe \& N. Note, pp. 169-211. World Scientific. [DA]

Aerts, D. \& Aerts, S. (1995) Applications of quantum statistics in psychological studies of decision processes. Foundations of Science 1:85-97. [DA, HA, HRN, aEMP]

Aerts, D., Aerts, S., Broekaert, J. \& Gabora, L. (2000) The violation of Bell inequalities in the macroworld. Foundations of Physics 30(9):1387-414. [DA]

Aerts, D. \& D’Hooghe, B. (2009) Classical logical versus quantum conceptual thought: Examples in economics, decision theory and concept theory. Lecture Notes in Computer Science 5494:128-42. [DA]

Aerts, D., Durt, T., Grib, A., Van Bogaert, B. \& Zapatrin, A. (1993) Quantum structures in macroscopical reality. International Journal of Theoretical Physics 32:489-98. [DA]

Aerts, D. \& Gabora, L. (2005a) A theory of concepts and their combinations I: The structure of the sets of contexts and properties. Kybernetes 34:167-91. [DA]

Aerts, D. \& Gabora, L. (2005b) A theory of concepts and their combinations II: A Hilbert space representation. Kybernetes 34:192-221. [DA, arEMP]
Aerts, D., Gabora, L. \& Sozzo, S. (in press) Concepts and their dynamics: A quantumtheoretic modeling of human thought. Topics in Cognitive Science. [DA]

Aerts, D. \& Sozzo, S. (2011a) Quantum structure in cognition: Why and how concepts are entangled. Lecture Notes in Computer Science 7052:116-27. [DA]

Aerts, D. \& Sozzo, S. (2011b) Quantum structure in cognition: Why and how concepts are entangled. In: Proceedings of the Quantum Interaction Conference, pp. 118-29. Springer. [AK, arEMP]

Alxatib, S. \& Pelletier, J. (2011) On the psychology of truth-gaps. In: Vagueness in communication, ed. R. Nouwen, R. van Rooij, U. Sauerland \& H.-C. Schmitz, pp. 13-36. Springer-Verlag. [rEMP]

Andersen, S., Harrison, G., Lau, M. \& Rutström, E. (2011) Discounting behavior: A reconsideration. CEAR Working Paper 2011-03. Available at: http://cear.gsu.edu/ files/Discounting\%20Behavior\%20-\%20A\%20Reconsideration.pdf. [DR]

Anderson, J. R. (1990) The adaptive character of thought. Erlbaum. [PK, aEMP]

Anderson, J. R. (1991) The adaptive nature of human categorization. Psychological Review 98:409-29. [PK, aEMP]

Anderson, J. R. (2007) Using brain imaging to guide the development of a cognitive architecture. In: Integrated models of cognitive systems, ed. W. D. Gray, pp. 4962. Oxford University Press. [CG]

Anderson, J. R., Bothell, D., Byrne, M. D., Douglass, S., Lebiere, C. \& Qin, Y. (2004) An integrated theory of the mind. Psychological Review 111:1036-60. [JNM]

Anderson, J. R. \& Lebiere, C. (1998) The atomic components of thought. Lawrence Erlbaum Associates. [CG]

Anderson, J. R. \& Lebiere, C. (2003) The Newell Test for a theory of cognition. Behavioral and Brain Sciences 26:587-640. [CG, JNM]

Anderson, J. R. \& Schooler, L. J. (1991) Reflections of the environment in memory. Psychological Science 2:396-408. [JNM]

Anderson, N. (1971) Integration theory and attitude change. Psychological Review 78:171-206. [aEMP]

Ashby, F. G. \& Gott, R. E. (1988) Decision rules in the perception and categorization of multidimensional stimuli. Journal of Experimental Psychology: Learning, Memory, \& Cognition 14:33-53. [RCG]

Ashby, F. G. \& Maddox, W. T. (2005) Human category learning. Annual Reviews of Psychology 56:149-78. [RCG]

Ashby, F. G. \& Perrin, N. A. (1988) Towards a unified theory of similarity and recognition. Psychological Review 95:124-50. [aEMP]

Aspect, A. (1999) Bell's inequality tests: More ideal than ever. Nature 398:189-90. [END]

Aspect, A., Graingier, P. \& Roger, G. (1981) Experimental tests of realistic local theories via Bell's theorem. Physical Review Letters 47:460-67. [rEMP]

Atmanspacher, H. (2004) Quantum theory and consciousness: An overview with selected examples. Discrete Dynamics 8:51-73. [aEMP]

Atmanspacher, H. \& beim Graben, P. (2007) Contextual emergence of mental states from neurodynamics. Chaos and Complexity Letters 2:151-68. [HA]

Atmanspacher, H. \& Filk, T. (2010) A proposed test of temporal nonlocality in bistable perception. Journal of Mathematical Psychology 54:314-21. [HA, arEMP]

Atmanspacher, H., Filk, T. \& Romer, H. (2004) Quantum zero features of bistable perception. Biological Cybernetics 90:33-40. [aEMP]

Atmanspacher, H. \& Römer, H. (2012) Order effects in sequential measurements of non-commuting psychological observables. Journal of Mathematical Psychology 56:274-80. [HA, arEMP]

Atmanspacher, H., Römer, H. \& Walach, H. (2002) Weak quantum theory: Complementarity and entanglement in physics and beyond. Foundations of Physics 32:379-406. [HA, RB, aEMP]

Ausubel, D. P. (1963) The psychology of meaningful verbal learning. Grune \& Stratton. [AC]

Ausubel, D. P. (1968) Educational psychology: A cognitive view. Holt, Rinehart \& Winston. [AC]

Axelrod, R. \& W. Hamilton, D. (1981) The evolution of cooperation. Science 211:1390-96. [CB]

Baaquie, B. E. (2004) Quantum finance: Path integrals and Hamiltonians for options and interest rates. Cambridge University Press. [aEMP]

Balakrishnan, J. D. (1998) Measures and interpretations of vigilance performance: Evidence against the detection criterion. Human Factors 40:601-23. [MVCB]

Baltag, A. \& Smets, S. (2005) Complete axiomatization for quantum action. International Journal of Theoretical Physics 44(12):2267-81. [RB]

Banerjee, A., Pillai, A. S., Sperling, J. R., Smith, J. F. \& Horwitz, B. (2012a) Temporal microstructure of cortical networks (TMCN) underlying task-related differences. NeuroImage 62:1643-57. [AB]

Banerjee, A., Tognoli, E., Assisi, C. G., Kelso, J. A. \& Jirsa, V. K. (2008) Mode level cognitive subtraction (MLCS) quantifies spatiotemporal reorganization in largescale brain topographies. NeuroImage 42(2):663-74. [AB]

Banerjee, A., Tognoli, E., Kelso, J. A. \& Jirsa, V. K. (2012b) Spatiotemporal reorganization of large-scale neural assemblies underlies bimanual coordination. Neuroimage 62(3):1582-92. [AB]

Bao, L., Hogg, K. \& Zollman, D. (2002) Model analysis of dine structure of student models: An example with Newton's Third Law. American Journal of Physics 70:755-78. [DRF] 
Bar-Hillel, M. \& Neter, E. (1993) How alike is it versus how likely is it: A disjunction fallacy in probability judgments. Journal of Personality and Social Psychology 65:1119-31. [aEMP]

Barkan, R. \& Busemeyer, J. R. (2003) Modeling dynamic inconsistency with a changing reference point. Journal of Behavioral Decision Making 16:235-55 [aEMP]

Baron, J. (2004) Normative models of judgment and decision making. In: Blackwell handbook of judgment and decision making, ed, D. J. Koehler \& N. Harvey, pp. 19-36. Blackwell. [JS]

Baron, J. (2008) Thinking and deciding, 4th ed. Cambridge University Press. [ELK]

Barsalou, L. W. (2008) Grounded cognition. Annual Review of Psychology 59:617645. [RB]

Barwise, J. \& Perry, J. (1983) Situations and attitudes. MIT Press. [MO]

Basoalto, R. M. \& Percival, I. C. (2003) BellTest and CHSH experiments with more than two settings. Journal of Physics A: Mathematical \& General 36:7411-23. [END]

Bawden, D. (2008) Smoother pebbles and the shoulders of giants: The developing foundations of information science. Journal of Information Science 34(4), 415-26. [AC]

Bawden, D. (2011) Brookes equation: The basis for a qualitative characterization of information behaviours. Journal of Information Science 37(1):101-108. $[\mathrm{AC}]$

Beckmann, C. F., DeLuca, M., Devlin, J. T. \& Smith, S. M. (2005) Investigations into resting-state connectivity using independent component analysis. Philosophical Transactions of the Royal Society of London B 360:1001-13. [AB]

beim Graben, P. \& Atmanspacher, H. (2009) Extending the philosophical significance of the idea of complementarity. In: Recasting reality. Wolfgang Pauli's philosophical ideas and contemporary science, ed. H. Atmanspacher \& H. Primas, pp. 99-113. Springer. [RB]

beim Graben, P., Filk, T. \& Atmanspacher, H. (2013) Epistemic entanglement due to non-generating partitions of classical dynamical systems. International Journal of Theoretical Physics 52:723-34. [HA]

Bell, J. (1964) On the Einstein-Podolsky-Rosen paradox. Physics 1:195-200. [DA, END]

Berg, N. \& Gigerenzer, G. (2010) As-if behavioral economics: Neoclassical economics in disguise? History of Economic Ideas 18:133-66. [JNM]

Bergus, G. R., Chapman, G. B., Levy, B. T., Ely, J. W. \& Oppliger, R. A. (1998) Clinical diagnosis and order information. Medical Decision Making 18:412 17. [aEMP]

Binmore, K. (2009) Rational decisions. Princeton University Press. [DR]

Birnbaum, M. H. (2008) New paradoxes of risky decision making. Psychological Review 115:463-501. [aEMP, TR]

Blutner, R. (2009) Concepts and bounded rationality: An application of Niestegge's approach to conditional quantum probabilities. In: Foundations of probability and physics-5, ed. L. E. A. Acardi, G. Adenier, C. Fuchs, G. Jaeger, A. Y. Khrennikov, J.-Å. Larsson \& S. Stenholm, pp. 302-10. American Institute of Physics Conference Proceedings. [aEMP]

Blutner, R. (2012) Questions and answers in an orthoalgebraic approach. Journal of Logic, Language and Information 21(3):237-77. [RB]

Blutner, R., Pothos, E. M. \& Bruza, P. (in press) A quantum probability perspective on borderline vagueness. Topics in Cognitive Science [rEMP]

Bordley, R. F. (1998) Quantum mechanical and human violations of compound probability principles: Toward a generalized Heisenberg uncertainty principle. Operations Research 46:923-26. [aEMP]

Brainerd, C. J. \& Reyna, V. F. (2008) Episodic over-distribution: A signature effect of familiarity without recognition. Journal of Memory \& Language 58:765-86. [aEMP]

Brainerd, C. J., Reyna, V. F. \& Ceci, S. J. (2008) Developmental reversals in false memory: A review of data and theory. Psychological Bulletin 134:343-82. [aEMP]

Brainerd, C. J., Reyna, V. F. \& Mojardin, A. H. (1999) Conjoint recognition. Psychological Review 106:160-79. [aEMP]

Brandstätter, E., Gigerenzer, G. \& Hertwig, R. (2006) The priority heuristic: Making choices without trade-offs. Psychological Review 113:409-32. [JNM]

Brase, G. L. \& Shanteau, J. (2011) The unbearable lightness of “Thinking”: Moving beyond simple concepts of thinking, rationality, and hypothesis testing. Behavioral \& Brain Sciences 34:250-51. [JS]

Bröder, A. \& Gaissmaier, W. (2007) Sequential processing of cues in memory-based multi-attribute decisions. Psychonomic Bulletin \& Review 14:895-900. [JNM]

Brookes, B. C. (1980) The foundations of information science, Part 1. Philosophical aspects Journal of Information Science 2(3/4):125-34. [AC]

Brookes, B. C. (1981) Information technology and the science of information. In: Information retrieval research, ed. R. N. Oddy, S. E. Robertson, C. J. van Rijsbergen \& P. W. Williams, pp. 1-8. Butterworths. [AC]

Brown, H. D. (2000) Principles of language learning and teaching. Addison Wesley Longman, Inc. [AC]
Brunswik, E. (1964) Scope and aspects of the cognitive problem. In: Contemporary approaches to cognition, ed. J. S. Bruner, E. Brunswik, L. Festinger, F. Heider, K. F. Muenzinger, C. E. Osgood \& D. Rapaport, pp. 5-31. Harvard University Press. [JNM]

Bruza, P., Kitto, K. \& McEvoy, D. (2008) Entangling words and meaning. In: Proceedings of the Second Quantum Interaction Conference, pp. 118-24. Springer. [rEMP]

Bruza, P., Kitto, K., Nelson, D. \& McEvoyc, C. (2009) Is there something quantumlike about the human mental lexicon? Journal of Mathematical Psychology 53:362-77. [AK, rEMP]

Bruza, P., Kitto, K., Ramm, B. \& Sitbon, L. (2012) A probabilistic framework for analyzing the compositionality of conceptual combinations. Under review. [HA]

Bruza, P. D., Kitto, K., Nelson, D. \& McEvoy, C. L. (2009) Is there something quantum-like about the human mental lexicon? Journal of Mathematical Psychology 53:362-77. [aEMP]

Bub, J. (1999) Interpreting the quantum world. Cambridge University Press. [rEMP]

Bullmore, E. \& Sporns, O. (2009) Complex brain networks: Graph theoretical analysis of structural and functional systems. Nature Review Neuroscience 10 (3):186-98. [AB]

Busemeyer, J. R. \& Bruza, P. D. (2012) Quantum models of cognition and decision Cambridge University Press. [HA, RB, MDL, arEMP]

Busemeyer, J. R., Matthew, M. \& Wang, Z. A. (2006a) Quantum game theory explanation of disjunction effects. In: Proceedings of the 28th Annual Conference of the Cognitive Science Society, ed. R. Sun \& N. Miyake, pp. 131-35. Erlbaum. [aEMP]

Busemeyer, J. R., Pothos, E. M., Franco, R. \& Trueblood, J. S. (2011) A quantum theoretical explanation for probability judgment errors. Psychological Review 118(2):193-218. [PK, TJP, arEMP, KT]

Busemeyer, J. R., Wang, J., \& Shiffrin, R. M. (2012) Bayesian model comparison of quantum versus traditional models of decision making for explaining violations of the dynamic consistency principle. Paper presented at Foundations and Applications of Utility, Risk and Decision Theory, Atlanta, Georgia. [arEMP]

Busemeyer, J. R., \& Wang, Z. (2007) Quantum information processing explanation for interactions between inferences and decisions. In: Quantum Interaction, AAAI Spring Symposium, Technical Report SS-07-08, ed. P. D Bruza, W. Lawless, K. van Rijsbergen \& D. A. Sofge, pp. 91-97. AAAI Press [PK]

Busemeyer, J. R., Wang, Z. \& Lambert-Mogiliansky, A. (2009) Comparison of Markov and quantum models of decision making. Journal of Mathematical Psychology 53:423-33. [aEMP]

Busemeyer, J. R., Wang, Z. \& Townsend, J. T. (2006) Quantum dynamics of human decision-making. Journal of Mathematical Psychology 50:220-41. [PK, $\operatorname{arEMP]}$

Bush, R. R. \& Mostellar, F. (1955) Stochastic models for learning. Blackwell. [TR]

Cacioppo, J. T. \& Petty, R. E. (1982) The need for cognition. Journal of Personality and Social Psychology 42:116-31. [ELK]

Carlson, B. W. \& Yates, J. F. (1989) Disjunction errors in qualitative likelihood judgment. Organizational Behavior and Human Decision Processes 44:36879. [aEMP]

Carter, A. H. (2001) Classical and statistical thermodynamics. Prentice-Hall. [AC]

Cartwright, N. (1999) The dappled world: A study of the boundaries of science. Cambridge University Press. [CB]

Castro, A. (2013) The thermodynamic cost of fast thought. Minds and Machines. DOI: 10.1007/s11023-013-9302-x. [AC]

Chalmers, D. (1995) Facing up to the problem of consciousness. Journal of Consciousness Studies 2:200-19. [DM]

Charnov, E. L. (1976) Optimal foraging, the marginal value theorem. Theoretical Population Biology 9:129-36. [RCG]

Chater, N. \& Oaksford, M. (2012) Normative systems: Logic, probability, and rational choice. In: The Oxford handbook of thinking and reasoning, ed. K. Holyoak \& R. Morrison, pp. 11-21. Oxford University Press. [MO]

Chefles, A. (2000) Quantum state discrimination. Contemporary Physics 41(6):40124. [AK]

Chomsky, N. (1995) The minimalist program. MIT Press. [RB]

Chomsky, N. (2005) Three factors in language design. Linguistic Inquiry 36(1):122. $[\mathrm{RB}]$

Choo, F.-X. \& Eliasmith, C. (2010) A spiking neuron model of serial-order recall. In: Proceedings of the 32nd Annual Conference of the Cognitive Science Society, ed S. Ohlsson \& R. Cattrambone, pp. 2188-93. Cognitive Science Society. [TCS]

Clauser, J. \& Horne, M. (1974) Experimental consequences of objective local theories. Physical Review D 10:526-35. [rEMP]

Clauser, J. F., Horne, M. A., Shimony, A. \& Holt, R. A. (1969) Proposed experiment to test local hidden-variable theories. Physical Review Letters 23:880-84. [END] 
Cole, C. (1997) Calculating the information content of an information process for a domain expert using Shannon's mathematical theory of communication: A preliminary analysis. Information Processing and Management 33(6):715-26. [AC]

Cole, C. (2011) A theory of information need for information retrieval that connects information to knowledge. Journal of the American Society for Information Science and Technology 62(7):1216-31. [AC]

Collins, G. P. (2006) Computing with quantum knots. Scientific American 294(4):5663. $[\mathrm{SRH}]$

Conte, E., Khrennikov, A. Y., Todarello, O., Federici, A., Mendolicchio, L. \& Zbilut, J. P. (2009) Mental states follow quantum mechanics during perception and cognition of ambiguous figures. Open Systems and Information Dynamics 16:117. [arEMP]

Cornelius, I. (2002) Theorizing information for information science. Annual Review of Information Science and Technology 36:393-425. [AC]

Corr, P. J. (2011) Anxiety: Splitting the phenomenological atom. Personality and Individual Differences 50:889-97. [PJC]

Costello, F. J. (2009) How probability theory explains the conjunction fallacy. Journal of Behavioral Decision Making 22:213-34. [KT]

Craddock, T. J. A., St. George, M., Freedman, H., Barakat, K. H., Damaraju, S., Hameroff, S. \& Tuszynski, J. A. (2012a) Computational predictions of volatile anesthetic interactions with the microtubule cytoskeleton: Implications for side effects of general anesthesia. PLoS ONE 7(6):e37251. [SRH]

Craddock, T. J. A., Tuszynski, J. A. \& Hameroff, S. (2012b) Cytoskeletal signaling: Is memory encoded in microtubule lattices by CaMKII phosphorylation? PLoS Computational Biology 8(3):e1002421. [SRH]

Croson, R. (1999) The disjunction effect and reason-based choice in games. Organizational Behavior and Human Decision Processes 80:118-33. [aEMP]

Crupi, V., Fitelson, B. \& Tentori, K. (2008) Probability, confirmation, and the conjunction fallacy. Thinking \& Reasoning 14:182-99. [KT]

de Barros, J. A. \& Suppes, P. (2009) Quantum mechanics, interference, and the brain. Journal of Mathematical Psychology 53:306-13. [aEMP]

de Finetti, B., Machi, A. \& Smith, A. (1993) Theory of probability: A critical introductory treatment. Wiley. [aEMP]

De Fraja, G. (2009) The origin of utility: Sexual selection and conspicuous consumption. Journal of Economic Behavior \& Organisation 72:51-69. [RCG]

Debaere, F., Swinnen, S. P., Beatse, E., Sunaert, S., Van Hecke, P. \& Duysens, J (2001) Brain areas involved in interlimb coordination: a distributed network. Neuroimage 14(5):947-58. [AB]

DeCarlo, L. T. (1998) Signal detection theory and generalized linear models. Psychological Methods 3:186-205. [MVCB]

Dirac, P. (1958) Quantum mechanics, 4th ed. Oxford University Press. [DRF]

Dirac, P. A. M. (1999) The principles of quantum mechanics (International series of monographs on physics). Oxford University Press. [AC]

Dougherty, M. R. P., Gettys, C. F. \& Ogden, E. E. (1999) Minerva-DM: A memory processes model for judgments of likelihood. Psychological Review 106:180209. [JNM]

Dunwoody, P. T. (2009) Theories of truth as assessment criteria in judgment and decision making. Judgment and Decision Making 4:11625. [BRN]

Dzhafarov, E. N. (2003) Selective influence through conditional independence. Psychometrika 68:7-26. [END]

Dzhafarov, E. N. \& Kujala, J. V. (2010) The joint distribution criterion and the distance tests for selective probabilistic causality. Frontiers in Quantitative Psychology and Measurement 1:151. [END]

Dzhafarov, E. N. \& Kujala, J. V. (2012a) Quantum entanglement and the issue of selective influences in psychology: An overview. Lecture Notes in Computer Science 7620: 184-95. [END]

Dzhafarov, E. N. \& Kujala, J. V. (2012b) Selectivity in probabilistic causality: Where psychology runs into quantum physics. Journal of Mathematical Psychology 56:54-63. [END]

Dzhafarov, E. N. \& Kujala, J. V. (in press a) All-possible-couplings approach to measuring probabilistic context. PLOS ONE (available as arXiv:1209.3430 [math.PR]. [END]

Dzhafarov, E. N. \& Kujala, J. V. (in press b) Order-distance and other metric-like functions on jointly distributed random variables. Proceedings of the American Mathematical Society. (available as arXiv:1110.1228 [math.PR]). [END]

Edelman, G. M. \& Gally, J. A. (2001) Degeneracy and complexity in biological systems. Proceedings of the National Academy of Sciences of the United States America 98(24):13,763-68. [AB]

Einstein, A., Podolsky, B. \& Rosen, N. (1935) Can quantum-mechanical description of physical reality be considered complete? Physical Review 47:777-80. [DA]

Eliasmith, C. (2005) Cognition with neurons: A large-scale, biologically realistic model of the Wason task. In: Proceedings of the 27th Annual Meeting of the Cognitive Science Society, ed. B. Bara, L. Barsalou \& M. Bucciarelli, pp. 62429. Cognitive Science Society. [TCS]

Eliasmith, C. (in press) How to build a brain: A neural architecture for biological cognition. Oxford University Press. [TCS]

Eliasmith, C. \& Anderson, C. H. (2003) Neural engineering: Computation, representation and dynamics in neurobiological systems. MIT Press. [TCS]
Elqayam, S. \& Evans, J. St. B. T. (2011) Subtracting “ought” from “is”: Descriptivism versus normativism in the study of human thinking. Behavioral \& Brain Sciences 34(5):233-48. [MO, JS]

Engel, G. S., Calhoun, T. R., Read, E. L., Ahn, T. K., Mancal, T., Cheng, Y. C., Blankenship, R. E. \& Fleming, G. R. (2007) Evidence for wavelike energy transfer through quantum coherence in photosynthetic systems. Nature (London) 446(7137):782-86. [SRH, DM]

Fano, U. (1957) Description of states in quantum mechanics by density matrix and operator techniques. Reviews of Modern Physics 29:74-93. [DRF]

Fantino, E. \& Esfandiari, A. (2002) Probability matching: Encouraging optimal responding in humans. Canadian Journal of Experimental Psychology, 56:5863. [RCG]

Feldman, J. M. \& Lynch, J. G. (1988) Self-generated validity and other effects of measurement on belief, attitude, intention, and behavior. Journal of Applied Psychology 73:421-35. [aEMP]

Festinger, L. (1957) A theory of cognitive dissonance. Stanford University Press. [arEMP]

Feynman, R. P. (1965) The character of physical law. Random House, p. 129. [HA]

Feynman, R. P. (1988) QED: The strange theory of light and matter. Princeton University Press. [DA]

Fine, A. (1982) Joint distributions, quantum correlations, and commuting observables. Journal of Mathematical Physics 23:1306-10. [END, aEMP]

Fodor, J. A. (1983) The modularity of mind. The MIT Press. [aEMP]

Fox, C. R. \& Levav, J. (2000) Familiarity bias and belief reversal in relative likelihood judgments. Organizational Behavior and Human Decision Processes, 82:26892. [PK]

Franco, R. (2009) The conjunction fallacy and interference effects. Journal of Mathematical Psychology 53:415-22. [AK]

Freedman, M. H., Kitaev, A., Larsen, M. J. \& Wang, Z. (2002) Topological quantum computation. Bulletin of the American Mathematical Society 40:31-38. [SRH]

Friston, K. J., Harrison, L. \& Penny, W. (2003) Dynamic causal modelling. NeuroImage 19:1273-302. [AB]

Fuss, I. \& Navarro, D. J. (in press) Open parallel cooperative and competitive decision processes: A potential provenance for quantum probability decision models. Topics in Cognitive Science. [DJN]

Gabora, L. \& Aerts, D. (2002) Contextualizing concepts using a mathematical generalization of the quantum formalism. Journal of Experimental and Theoretical Artificial Intelligence 14:327-58. [DA]

Gasset, J. O. (1998) Meditaciones del Quijote. Catedra. [AC]

Gauger, E. M., Rieper, E., Morton, J. J. L., Benjamin, S. C. \& Vedral, V. (2011) Sustained quantum coherence and entanglement in the avian compass. Physical Review Letters 106:040503. [SRH]

Gavanski, I. \& Roskos-Ewoldsen, D. R. (1991) Representativeness and conjoint probability. Journal of Personality and Social Psychology 61:181-94. [aEMP]

Gayler, R. W. (2003) Vector Symbolic Architectures answer Jackendoff's challenges for cognitive neuroscience. In: ICCS/ASCS International Conference on Cognitive Science, ed. P. Slezak, pp. 133-38. University of New South Wales. [TCS]

Georgopoulos, A. P., Schwartz, A. B. \& Kettner, R. E. (1986) Neuronal population coding of movement direction. Science 233(4771):1416-19. [TCS]

Gigerenzer, G. (1996) On narrow norms and vague heuristics: A reply to Kahneman and Tversky. Psychological Review 103:592-96. [JNM]

Gigerenzer, G. (1997) Bounded rationality models of fast and frugal inference. Swiss Journal of Economics and Statistics 133:201-18. [RB]

Gigerenzer, G., Hoffrage, U. \& Kleinbölting, H. (1991) Probabilistic mental models: A Brunswikian theory of confidence. Psychological Review 98:506-28. [JNM]

Gigerenzer, G. \& Selten, R., ed. (2001) Bounded rationality: The adaptive toolbox. MIT Press. [JNM]

Gigerenzer, G. \& Todd, P. M. (1999) Simple heuristics that make us smart. Oxford University Press. [aEMP]

Gleason, A. M. (1957) Measures on the closed subspaces of a Hilbert space. Journal of Mathematics and Mechanics 6:885-94. [RB]

Glöckner, A. \& Betsch, T. (2011) The empirical content of theories in judgment and decision making: Shortcomings and remedies. Judgment and Decision Making 6:711-21. [TR]

Gokhale, A. A. (2004) Introduction to telecommunications (2nd ed.). Thomson Delmar Learning. [AC]

Goldstone, R. L. (1994) Similarity, interactive activation, and mapping. Journal of Experimental Psychology: Learning, Memory and Cognition 20:3-28. [aEMP]

Gonzalez, C. (2013). The boundaries of Instance-Based Learning Theory for explaining decisions from experience. pp. 73-98. In Decision making: Neural and behavioural approaches. Vol. 202. ed. V. S. C. Pammi \& N. Srinivasan, Progress in Brain Research. Elsevier. ISBN 978-0-444-62604-2. [CG]

Gonzalez, C. \& Dutt, V. (2011) Instance-based learning: Integrating decisions from experience in sampling and repeated choice paradigms. Psychological Review 118(4):523-51. [CG] 
References/Pothos \& Busemeyer: Can quantum probability provide a new direction for cognitive modeling?

Green, D. M. \& Swets, J. A. (1966) Signal detection theory and psychophysics. Wiley. [MVCB]

Griffiths, R. B. (2003) Consistent quantum theory. Cambridge University Press. [aEMP]

Griffiths, T. L., Chater, N., Kemp, C., Perfors, A. \& Tenenbaum, J. B. (2010) Probabilistic models of cognition: Exploring representations and inductive biases. Trends in Cognitive Sciences 14:357-64. [HRN, aEMP]

Griffiths, T. L., Kemp, C. \& Tenenbaum, J. B. (2008) Bayesian models of cognition. In: Cambridge handbook of computational cognitive modeling, ed. R. Sun, pp. 59-100. Cambridge University Press. [AK, JNM]

Grover, L. K. (1997) Quantum mechanics helps in searching for a needle in a haystack. Physical Review Letters 79:325-28. [aEMP]

Gudder, S .P. (1988) Quantum probability. Academic Press. [HA]

Haack, S. (1974) Deviant logic. Cambridge University Press. [MO]

Haack, S. (1978) Philosophy of logics. Cambridge University Press. [MO]

Hagan, S., Hameroff, S. \& Tuszynski, J. (2002) Quantum computation in brain microtubules? Decoherence and biological feasibility. Physical Review E 65:061901. [SRH, DM]

Hahn, U., Chater, N. \& Richardson, L. B. (2003) Similarity as transformation. Cognition 87:1-32. [aEMP]

Hameroff, S. (1998) Quantum computation in brain microtubules? The PenroseHameroff "Orch OR" model of consciousness. Philosophical Transactions of the Royal Society of London Series A 356:1869-96. [SRH]

Hameroff, S. (2006a) Consciousness, neurobiology and quantum mechanics: The case for a connection, In: The Emerging Physics of Consciousness, ed. J. Tuszynski, pp. 193-252, Springer. [SRH]

Hameroff, S. (2006b) The entwined mysteries of anesthesia and consciousness. Anesthesiology 105:400-12. [SRH]

Hameroff, S. (2010) The "conscious pilot" - dendritic synchrony moves through the brain to mediate consciousness. Journal of Biological Physics 36:71-93. $[\mathrm{SRH}]$

Hameroff, S. (2012) How quantum brain biology can rescue conscious free will. Frontiers in Integrative Neuroscience 6(93):1-17. DOI: 10.3389/ fnint.2012.00093. [SRH]

Hameroff, S., Nip, A., Porter, M. \& Tuszynski, J. (2002) Conduction pathways in microtubules, biological quantum computation and microtubules. Biosystems 64(13):149-68. [SRH]

Hammeroff, S. R. (1998) Quantum computation in brain microtubules? The Penrose-Hammeroff "orch-or" model of consciousness. Philosophical Transactions of the Royal Society A 356:1869-96. [DJN, aEMP]

Hameroff, S. R. (2007) The brain is both neurocomputer and quantum computer Cognitive Science 31:1035-45. [SRH, aEMP]

Hameroff, S. R. \& Penrose, R. (1996a) Conscious events as orchestrated spacetime selections. Journal of Consciousness Studies 3(1):36-53. [SRH]

Hameroff, S. R. \& Penrose, R. (1996b) Orchestrated reduction of quantum coherence in brain microtubules: A model for consciousness. Mathematics and Computers in Simulation 40:453-80. [SRH]

Hammond, K. R. (1996) Human judgment and social policy. Irreducible uncertainty, inevitable error, unavoidable injustice. Oxford University Press. [BRN]

Hampton, J. A. (1987) Inheritance of attributes in natural concept conjunctions. Memory \& Cognition 15:55-71. [JAH]

Hampton, J. A. (1988a) Disjunction of natural concepts. Memory \& Cognition 16:579-91. [DA, aEMP]

Hampton, J. A. (1988b) Overextension of conjunctive concepts: Evidence for a unitary model for concept typicality and class inclusion. Journal of Experimental Psychology: Learning, Memory, and Cognition 14:12-32. [DA, JAH, aEMP]

Hampton, J. A. (1997) Emergent attributes in conceptual combinations. In: Creative thought: An investigation of conceptual structures and processes, ed. T. B. Ward, S. M. Smith \& J. Viad, pp. 83-110. American Psychological Association Press. [JAH]

Hampton, J. A. (2012) Thinking intuitively: The rich (and at times illogical) world of concepts. Current directions in psychological science 21:398-402. [JAH]

Harrison, G. \& Rutström, E. (2008) Risk aversion in the laboratory. In: Risk aversion in experiments, ed. J. Cox \& G. Harrison, pp. 41-196. Emerald. [DR]

Harrison, G. \& Rutström, E. (2009) Expected utility and prospect theory: One wedding and a decent funeral. Experimental Economics 12:133-58. [DR]

Hartl, J. A. \& Fantino, E. (1996) Choice as a function of reinforcement ratios in delayed matching to sample. Journal of the Experimental Analysis of Behavior 66:11-27. [RCG]

Hertwig, R., Hoffrage U. \& the ABC Research Group (2013) Simple heuristics in a social world. Oxford University Press. [JNM]

Hertwig, R., Hoffrage, U. \& Martignon, L. (1999) Quick estimation: Letting the environment do the work. In: Simple heuristics that make us smart, G. Gigerenzer, P. M. Todd \& the ABC Research Group, pp. 209-34. Oxford University Press. [JNM]

Hey, J. \& Orme, C. (1994) Investigating generalizations of expected utility theory using experimental data. Econometrica 62:1291-326. [DR]
Hirsh, J. B., Mar, R. A. \& Peterson, J. B. (2012) Psychological entropy: A framework for understanding uncertainty-related anxiety. Psychological Review 119:31420. [PJC]

Hogarth, R. M. \& Einhorn, H. J. (1992) Order effects in belief updating: The beliefadjustment model. Cognitive Psychology 24:1-55. [aEMP]

Holton, G. (1970) The roots of complementarity. Daedalus 99:1015-55. [HA]

Horwitz, B., Grady, C. L., Haxby, J. V., Ungerleider, L. G., Schapiro, M. B. \& Mishkin, M. (1992) Functional associations among human posterior extrastriate brain regions during object and spatial vision. Journal of Cognitive Neuroscience 4:311-22. [AB]

Houston, A. I., McNamara, J. M. \& Steer, M. D. (2007a) Do we expect natural selection to produce rational behaviour? Philosophical Transactions of the Royal Society 362:1531-43. [AIH]

Houston, A. I., McNamara, J. M. \& Steer, M. D. (2007b) Violations of transitivity under fitness maximization. Biology Letters 3:365-67. [AIH]

Hughes, G. (1989) The structure and interpretation of quantum mechanics. Harvard University Press. [TJP]

Hughes, R. I. G. (1989) The structure and interpretation of quantum mechanics. Harvard University Press. [MVCB, aEMP]

Hume, D. (1751/1999) An enquiry concerning human understanding. Oxford University Press. [CB]

Hursh, S. R. (1984) Behavioral economics. Journal of the Experimental Analysis of Behavior 42:435-52. [RCG]

Isham, C. J. (1989) Lectures on quantum theory. World Scientific. [arEMP]

Jacobson, N. S., Roberts, L. J., Berns, S. B. \& McGlinchey, J. B. (1999) Methods for defining and determining the clinical significance of treatment effects: Description, application, and alternatives. Journal of Consulting and Clinical Psychology 67:300-307. [JS]

Jaynes, E. T. (1993) A backward look to the future. In: Physics and probability, ed. W. T. Grandy, Jr. \& P. W. Milonni, pp. 261-75. Cambridge University Press. [MDL]

Jaynes, E. T. (2003) Probability theory: The logic of science. Cambridge University Press. [MDL]

Jibu, M. \& Yasue, K. (1995) Quantum brain dynamics and consciousness. Benjamins. [aEMP]

Johnson, E. J., Haubl, G. \& Keinan, A. (2007) Aspects of endowment: A query theory of value construction. Journal of Experimental Psychology: Learning, Memory and Cognition 33(3):461-73. [RB, aEMP]

Johnson, E.J., Schulte-Mecklenbeck, M. \& Willemsen, M. (2008) Process models deserve process data: Comment on Brandstätter, Gigerenzer \& Hertwig (2006) Psychological Review 115:263-72. [JNM]

Jones, M. \& Love, B. C. (2011) Bayesian fundamentalism or enlightenment? On the explanatory status and theoretical contributions of Bayesian models of cognition. Behavioral and Brain Sciences 34:169-231. [BCL, aEMP]

Kahneman, D. (2003) A perspective on judgment and choice: Mapping bounded rationality. American Psychologist 58(9):697-720. [TJP]

Kahneman, D., Slovic, P. \& Tversky, A. (1982) Judgment under uncertainty: Heuristics and biases. Cambridge University Press. [JNM, aEMP]

Kahneman, D. \& Tversky, A. (1979) Prospect theory: An analysis of decision under risk. Econometrica 47:263-91. [PK, aEMP]

Kaznatcheev, A. (submitted) Unifying quantum and Markov models of decision making. $[\mathrm{AK}]$

Kenrick, D. T., Griskevicius, V., Sundie, J., Li, N. P., Li, Y. J. \& Neuberg, S. L. (2009) Deep rationality: The evolutionary economics of decision making. Social Cognition 27:764-85. [RCG]

Khalil, E. L. (1997a) Buridan's ass, uncertainty, risk, and self-competition: A theory of entrepreneurship. Kyklos 50:147-63. [ELK]

Khalil, E. L. (1997b) Chaos theory versus Heisenberg's uncertainty: Risk, uncertainty and economic theory. American Economist 41:27-40. [ELK]

Khalil, E. L. (2000) Types of metaphor and identificational slips in economic discourse. Research in the History of Economic Thought and Methodology 18A:83105. [ELK

Khalil, E. L. (2010) The Bayesian fallacy: Distinguishing internal motivations and religious beliefs from other beliefs. Journal of Economic Behavior and Organization 75:268-80. [ELK]

Khrennikov, A. Y. (1999) Classical and quantum mechanics on information spaces with applications to cognitive, psychological, social and anomalous phenomena. Foundations of Physics 29:1065-98. [HA]

Khrennikov, A. Y. (2010) Ubiquitous quantum structure: From psychology to finance. Springer. [aEMP]

Kim, J. \& Horwitz, B. (2009) How well does Structural Equation Modeling reveal abnormal brain anatomical connections? An fMRI simulation study. Neuroimage 45:1190-98. [AB]

Kitaev, A. Y. (2003) Fault-tolerant quantum computation. Annals of Physics 303 (1):2-30; quant-ph/9707021. [SRH]

Knowlton, B. J., Morrison, R. G., Hummel, J. E. \& Holyoak, K. J. (2012) A neurocomputational model for relational reasoning. Trends in Cognitive Sciences 16:373-81. [HRN] 
Kolmogorov, A. N. (1933/1950) Foundations of the theory of probability. Chelsea Publishing Co. [aEMP]

Koselj, K., Schnitzler, H.-U. \& Siemers, B. M. (2011) Horseshoe bats make adaptive pre-selection decisions, informed by echo cues. Proceedings of the Royal Society B: Biological Sciences 278:3034-41. [RCG]

Krivan, V., Cressman, R. \& Schneider, C. (2008) The ideal free distribution: A review and synthesis of the game-theoretic perspective. Theoretical Population Biology 73:403-25. [RCG]

Krueger, J. I., DiDonato, T. E., \& Freestone, D. (2012) Social projection can solve social dilemmas. Psychological Inquiry 23:1-27. [aEMP]

Krumhansl, C. L. (1978) Concerning the applicability of geometric models to similarity data: The interrelationship between similarity and spatial density. Psychological Review 85:445-63. [aEMP]

Kuhn, T. S. (1996) The structure of scientific revolutions. University of Chicago Press. [RB]

Kujala, J. V. \& Dzhafarov, E. N. (2008) Testing for selectivity in the dependence of random variables on external factors. Journal of Mathematical Psychology 52:128-44. [END]

Kumagai, W. \& Hayashi, M. (2011) Quantum hypothesis testing for quantum Gaussian states: Quantum analogues of chi-square, $\mathrm{t}$ and $\mathrm{F}$ tests. Available as arXiv preprint arXiv: $1110.6255 \mathrm{v} 1 . \quad$ [DJN]

Kusev, P., Ayton, P., van Schaik, P., Tsaneva-Atanasova, K., Stewart, N. \& Chater, N. (2011) Judgments relative to patterns: How temporal sequence patterns affect judgments and memory. Journal of Experimental Psychology: Human Perception and Performance 37:1874-886. [PK]

Kusev, P., Tsaneva-Atanasova, K., van Schaik, P. \& Chater, N. (2012a) Modelling judgement of sequentially presented categories using weighting and sampling without replacement. Behavior Research Methods. 44:1129-34. [PK]

Kusev, P., van Schaik, P. \& Aldrovandi, S. (2012b) Preferences induced by accessibility: Evidence from priming. Journal of Neuroscience, Psychology, and Economics 5:250-58. [PK]

Kusev, P., van Schaik, P., Ayton, P., Dent, J. \& Chater, N. (2009) Exaggerated risk: Prospect theory and probability weighting in risky choice. Journal of Experimental Psychology: Learning, Memory, and Cognition 35:1487-505. [PK]

Ladyman, J. \& Ross, D. (2007) Everything must go. Oxford University Press. [DR]

Lambert-Mogiliansky, A., Zamir, S. \& Zwirn, H. (2009) Type indeterminacy: A model of the KT(Kahneman-Tversky)-man. Journal of Mathematical Psychology 53(5):349-61. [aEMP]

LeBoeuf, R. A. \& Shafir, E. (2003) Deep thoughts and shallow frames: On the susceptibility to framing effects. Journal of Behavioral Decision Making 16:7792. [ELK]

Lejarraga, T., Dutt, V. \& Gonzalez, C. (2012) Instance-based learning: A general model of repeated binary choice. Journal of Behavioral Decision Making 25 (2):143-53. [CG]

Li, S. \& Taplin, J. (2002) Examining whether there is a disjunction effect in prisoner's dilemma games. Chinese Journal of Psychology 44:25-46. [aEMP]

Lichtenstein, S. \& Slovic, P. (1971) Reversals of preference between bids and choices in gambling decisions. Journal of Experimental Psychology 89:46-55. [PK]

Lichtenstein, S. \& Slovic, P. (1973) Response-induced reversals of preference in gambling: An extended replication in Las Vegas. Journal of Experimental Psychology 101:16-20. [PK]

Lichtenstein, S., Slovic, P., Fischhoff, B., Layman, M. \& Combs, B. (1978) Judged frequency of lethal events. Journal of Experimental Psychology: Human Learning and Memory 4:551-78. [RN]

Litt, A., Eliasmith, C., Kroon, F. W., Weinstein, S. \& Thagard, P. (2006) Is the brain a quantum computer? Cognitive Science 30:593-603. [AK, aEMP]

Lo, A. \& Mueller, M. (2010) WARNING!: Physics envy may be hazardous to your wealth. Journal of Investment Management 8:13-63. [JS]

MacCallum, R. C., Zhang, S, Preacher, K. J. \& Rucker, D. D. (2002) On the practice of dichotomization of quantitative variables. Psychological Methods 7:19-40. [JS]

Mach, E. (1911) History and root of the principle of conservation of Energy. Open Court Publishing. [JS]

Macmillan, N. A. \& Creelman, C. D. (2005) Detection theory: A user's guide. Cambridge University Press. [MVCB]

Macphail, E. M. (1987) The comparative psychology of intelligence. Behavioral and Brain Sciences 10:645-56. [RCG]

Maddox, W. T., Ashby, F. G. \& Bohil, C. J. (2003) Delayed feedback effects on rulebased and information-integration category learning. Journal of Experimental Psychology: Learning, Memory \& Cognition 29:650-62. [RCG]

Maia, T. V. \& Frank, M. J. (2011) From reinforcement learning models to psychiatric and neurological disorders. Nature Neuroscience 14:154-62. [HRN]

Malkiel, B. (2011, December 14) Physics envy [Review of the book Models behaving badly]. Wall Street Journal. Retrieved September 9, 2012 from http://online.wsj. com/article/SB10001424052970203430404577094760894401548.html. [JS]

Marewski, J. N. \& Mehlhorn, K. (2011) Using the ACT-R architecture to specify 39 quantitative process models of decision making. Judgment and Decision Making 6(6):439-519. [CG, JNM]
Marewski, J. N., Pohl, R. F. \& Vitouch, O. (2010) Recognition-based judgments and decisions: Introduction to the special issue (Vol. 1). Judgment and Decision Making 5:207-15. [JNM]

Marewski, J. N. \& Schooler, L. J. (2011) Cognitive niches: An ecological model of strategy selection. Psychological Review 118(3):393-437. [CG, JNM]

Markman, A. B. \& Gentner, D. (1993) Splitting the differences: A structural alignment view of similarity. Journal of Memory and Language 32:517-35. [aEMP]

Marr, D. (1982) Vision: A computational investigation into the human representation and processing of visual information. W. H. Freeman. [DJN, aEMP]

McIntosh, A. R. (2004) Contexts and catalysts: A resolution of the localization and integration of function in the brain. Neuroinformatics 2:175-82. [AB]

McIntosh, A. R., Grady, C. L., Ungerleider, L. G., Haxby, J. V., Rapoport, S. I. \& Horwitz, B. (1994) Network analysis of cortical visual pathways mapped with PET. Journal of Neurosciences, 14:655-66. [AB]

McKemmish, L. K., Reimers, J. R., McKenzie, R. H., Mark, A. E. \& Hush, N. S. (2009) Penrose-Hameroff orchestrated objective-reduction proposal for human consciousness is not biologically feasible. Physical Review E. 80:021912 [SRH]

McKenzie, C. R. M., Lee, S. M. \& Chen, K. K. (2002) When negative evidence increases confidence: Change in belief after hearing two sides of a dispute. Journal of Behavioral Decision Making 15:1-18. [aEMP]

McNamara, J. M., Trimmer, P. C. \& Houston, A. I. (2012) The ecological rationality of state-dependent valuation. Psychological Review 119:114-19. [AIH]

McNaughton, N. \& Corr, P. J. (2004) A two-dimensional neuropsychology of defense: Fear/anxiety and defensive distance. Neuroscience and Biobehavioral Reviews 28:285-305. [PJC]

Mellers, B., Hertwig, R. \& Kahneman, D. (2001) Do frequency representations eliminate conjunction effects? An exercise in adversarial collaboration. Psychological Science 12:269-75. [TJP]

Mirowski, P. (1992) Do economists suffer from physics envy? Finnish Economic Papers 5:61-68. [JS]

Miyamoto, J. M., Gonzalez, R. \& Tu, S. (1995) Compositional anomalies in the semantics of evidence. In: Decision making from a cognitive perspective (Volume 32 of the Psychology of Learning and Motivation), ed. J. Busemeyer, R. Hastie \& D. Medin, pp. 319-83. Academic Press. [rEMP]

Molholm, S., Ritter, W., Javitt, D. C. \& Foxe, J. J. (2004) Multisensory visual-auditory object recognition in humans: a high-density electrical mapping study. Cerebral Cortex 14(4):452-65. [AB]

Moore, D. W. (2002) Measuring new types of question-order effects. Public Opinion Quarterly 66:80-91. [BRN, aEMP]

Moreira, M. A. (2011) Meaningful learning: From the classical to the critical view. Meaningful Learning Review 1:1-15. [AC]

Morrison, J. H. \& Baxter, M. G. (2012) The aging cortical synapse: Hallmarks and implications for cognitive decline. Nature Reviews Neuroscience 13:240-50. [HRN]

Neill, S. D. (1982) Brookes, Popper and objective knowledge, Journal of Information Science 4(1):33-39. [AC]

Nellen, S. (2003) The use of the "take-the-best" heuristic under different conditions, modelled with ACT-R. In: Proceedings of the fifth international conference on cognitive modelling, ed. F. Detje, D. Dörner \& H. Schaub, pp. 171-76. Universitätsverlag Bamberg. [JNM]

Newell, A. (1990) Unified theories of cognition. Harvard University Press. [CG]

Newell, B. R. (2013) Judgment under uncertainty. In: Oxford handbook of cognitive psychology, ed. D. Reisberg, pp. 603-17. Oxford University Press. [BRN]

Nielsen, M. A. \& Chuang, I. L. (2000) Quantum computation and quantum information. Cambridge University Press. [AK, arEMP]

Nilsson, H., Winman, A., Juslin, P. \& Hansson, G. (2009) Linda is not a bearded lady: Configural weighting and adding as the cause of extension errors. Journal of Experimental Psychology: General, 138:517-34. [KT]

Noori, H. R. \& Jäger, W. (2010) Neurochemical oscillations in the basal ganglia. Bulletin of Mathematical Biology 72:133-47. [HRN]

Noori, H. R., Spanagel, R. \& Hansson, A. C. (2012) Neurocircuitry for modeling drug effects. Addiction Biology 17:827-64. [HRN]

Noppeney, U., Friston, K. J. \& Price, C. J. (2004) Degenerate neuronal systems sustaining cognitive functions. Journal of Anatomy 205(6):433-42. [AB]

Nosofsky, R. M. (1984) Choice, similarity, and the context theory of classification. Journal of Experimental Psychology: Learning, Memory \& Cognition 10:10414. [aEMP

Novak, J. D. (2011) A theory of education: Meaningful learning underlies the constructive integration of thinking, feeling, and acting leading to empowerment for commitment and responsibility. Meaningful Learning Review 1(2):1-14. [AC]

Nowak, M. \& Sigmund, K. (1992) Tit for tat in heterogeneous populations. Nature, 355:250-53. [CB]

Oaksford, M. \& Chater, N. (1998) Rational models of cognition. Oxford University Press. [JNM]

Oaksford, M. \& Chater, N. (2007) Bayesian rationality: The probabilistic approach to human reasoning. Oxford University Press. [PK, aEMP, DR] 
References/Pothos \& Busemeyer: Can quantum probability provide a new direction for cognitive modeling?

Oaksford, M. \& Chater, N. (2009) Pre'cis of Bayesian rationality: The probabilistic approach to human reasoning. Behavioral and Brain Sciences 32:69-120. [PK, arEMP]

Osherson, D. \& Smith, E. (1981) On the adequacy of prototype theory as a theory of concepts. Cognition 9:35-58. [DA, JAH]

Pachur, T., Hertwig, R. \& Rieskamp, J. (2013) The mind as an intuitive pollster: Frugal search in social spaces. In: Simple heuristics in a social world, ed. R. Hertwig, U. Hoffrage \& the ABC Research Group, pp. 261-91. Oxford University Press. [JNM]

Pais, A. (1991) Neils Bohr's times: In physics, philosophy and polity. Oxford University Press. [DRF]

Parasuraman, R. \& Masalonis, A. J. (2000) Fuzzy signal detection theory: Basic postulates and formulas for analyzing human and machine performance. Human Factors 42:636-59. [MVCB]

Pastore, R. E., Crawley, E. J., Berens, M. S. \& Skelly, M. A. (2003) “Nonparametric” Á and other modern misconceptions about signal detection theory. Psychonomic Bulletin \& Review 10:556-69. [MVCB]

Penrose, R. (1989) The emperor's new mind. Oxford University Press. [DRF, SRH, aEMP]

Penrose, R. (1994) Shadows of the mind: a search for the missing science of consciousness. Oxford University Press. [SRH]

Penrose, R. (1996) On gravity's role in quantum state reduction. General Relativity Gravity 28:581-600. [SRH]

Penrose, R. (2004) The road to reality: A complete guide to the laws of the universe. Jonathan Cape. [SRH]

Penrose, R. \& Hameroff, S. (2011) Consciousness in the universe: Neuroscience, quantum space-time geometry and Orch OR theory. Journal of Cosmology 14:1-17. Available at: http://journalofcosmology.com/Consciousness160.html. [SRH]

Penrose, R. \& Hameroff, S. R. (1995) What gaps? Reply to Grush and Churchland. Journal of Consciousness Studies 2:98-112. [SRH]

Perfors, A., Tenenbaum, J. B., Griffiths, T. L. \& Xu, F. (2011) A tutorial introduction to Bayesian models of cognitive development. Cognition 120:302-21. [aEMP]

Piron, C. (1976) Foundations of quantum physics. WA Benjamin, Inc. [RB]

Plate, T. (2003) Holographic reduced representations. CSLI Publication. [TCS]

Pothos, E. M. \& Busemeyer, J. R. (2009) A quantum probability explanation for violations of "rational" decision theory. Proceedings of the Royal Society B 276:2171-78. [arEMP]

Pothos, E. M. \& Busemeyer, J. R. (2011) A quantum probability explanation for violations of symmetry in similarity judgments. In: Proceedings of the 32nd Annual Conference of the Cognitive Science Society, pp. 2848-54. LEA. [aEMP]

Price, C. J. \& Friston, K. J. (2002) Degeneracy and cognitive anatomy. Trends in Cognitive Sciences 6(10):416-21. [AB]

Primas, H. (2007) Non-Boolean descriptions of mind-matter systems. Mind and Matter 5:7-44. [HA]

Rachlin, H., Green, L., Kagel, J. H. \& Battalio, R. (1976) Economic demand theory and psychological studies of choice. In: The psychology of learning and motivation (Vol. 10), ed. G. H. Bower, pp. 129-54. Academic Press. [RCG]

Redei, M. \& Summers, S. J. (2007) Quantum probability theory. Studies in the History and Philosophy of Modern Physics 38:390-417. [HA, aEMP]

Reisen N., Hoffrage U. \& Mast, F. W. (2008) Identifying decision strategies in a consumer choice situation. Judgment and Decision Making 3:641-58. [JNM]

Reyna, V. F. (2008) A theory of medical decision making and health: Fuzzy trace theory. Medical Decision Making 28:850-65. [aEMP]

Reyna, V. F. \& Brainerd, C. J. (1995) Fuzzy-trace theory: An interim synthesis. Learning and Individual Differences 7:1-75. [aEMP]

Ricciardi, L. M. \& Umezawa, H. (1967) Brain and physics of many bodied problems. Kybernetik 4:44-48. [aEMP]

Richman, F. \& Bridges, D. S. (1999) A constructive proof of Gleason's theorem. Journal of Functional Analysis 162(2):287-312 [RB]

Robson, A. \& Samuelson, L. (2011) The evolution of decision and experienced utilities. Theoretical Economics 6:311-39. [RCG]

Roth, G. \& Dicke, U. (2005) Evolution of the brain and intelligence. Trends in Cognitive Sciences 9:250-57. [RCG]

Russell, B. (1921) The analysis of mind. George Allen \& Unwin. [DR]

Russell, B. (1918-1924/1956) Logic and knowledge. George Allen \& Unwin. [DR]

Sanborn, A. N., Griffiths, T. L. \& Navarro, D. J. (2010) Rational approximations to rational models: Alternative algorithms for category learning. Psychological Review 117:1144-67. [aEMP]

Sapolsky, R. (1997) The trouble with testosterone, and other essays on the biology of the human predicament. Scribner. [JS]

Sarovar, M., Ishizaki, A., Fleming, G. R. \& Whaley, B. K. (2010) Quantum entanglement in photosynthetic light-harvesting complexes. Nature Physics 6 (6):462-67. [SRH]

Savage, L. (1954) The foundations of statistics. Wiley. [aEMP]

Schabas, M. (1993) What's so wrong with physics envy? In: Non-natural social science: Reflecting on the enterprise of More Heat than Light, ed. N. de Marchi, pp. 45-53. Duke University Press. [JS]
Scholes, G. S. (2010) Quantum-coherent electronic energy transfer: Did nature think of it first? Journal of Physics and Chemistry Letters 1:2-8. [SRH]

Schooler, L. J. \& Hertwig, R. (2005) How forgetting aids heuristic inference. Psychological Review 112(3):610-28. [CG, JNM]

Schuman, H. \& Presser, S. (1981) Questions and answers in attitude surveys: Experiments on question form, wording, and content. Academic Press. [aEMP]

Schwarz, N. (2007) Attitude construction: Evaluation in context. Social Cognition 25:638-56. [aEMP]

Sebastian-Gonzalez, E., Botella, F., Sempere, R. A. \& Sanchez-Zapata, J. A. (2010) An empirical demonstration of the ideal free distribution: Little Grebes Tachybaptus ruficollis breeding in intensive agricultural landscapes. Ibis 152:643-50. [RCG]

Seel, N. M. (2012) Assimilation theory of learning. In: Encyclopedia of the Sciences of Learning, ed. N. M. Seel, pp. 324-26. Springer. [AC]

Seising, S. (2010) Cybernetics, system(s) theory, information theory and fuzzy sets and systems in the 1950s and 1960s. Information Sciences 180:4459-76. $[\mathrm{AC}]$

Shafer, G. \& Tversky, A. (1985) Languages and designs for probability judgment. Cognitive Science 9:309-39. [aEMP]

Shafir, E. \& Tversky, A. (1992) Thinking through uncertainty: nonconsequential reasoning and choice. Cognitive Psychology 24:449-74. [DJN, aEMP]

Shafir, E. \& Tversky, A. (2004) Thinking through uncertainty: Nonconsequential reasoning and choice. In: Preference, belief, and similarity: Selected writings by Amos Tversky, ed. E. Shafir, pp. 703-27. MIT Press. [CB]

Shafir, E. B., Smith, E. E. \& Osherson, D. N. (1990) Typicality and reasoning fallacies. Memory \& Cognition 18:229-39. [rEMP]

Shanteau, J. C. (1970) An additive model for sequential decision making. Journal of Experimental Psychology 85:181-191. [aEMP]

Shepard, R. N. (1987) Toward a universal law of generalization for psychological science. Science 237(4820):1317-23. [TJP]

Shepard, R. N. (1994) Perceptual-cognitive universals as reflections of the world. Psychonomic Bulletin \& Review 1:2-28. [RCG]

Sher, S. \& McKenzie, C. R. M. (2008) Framing effects and rationality. In: The probabilistic mind: Prospects for Bayesian cognitive science, ed. N. Chater \& M Oaksford, pp. 79-96. Oxford University Press. [aEMP]

Shin, L. M. \& Liberzon, I. (2010) The neurocircuitry of fear, stress, and anxiety disorders. Neuropsychopharmacology 35:169-91. [HRN]

Shultz, T. R. (2007) The Bayesian revolution approaches psychological development Developmental Science 10:357-64. [AK]

Sides, A., Osherson, D., Bonini, N. \& Viale, R. (2002) On the reality of the conjunction fallacy. Memory and Cognition 30:191-98. [aEMP]

Simon, H. A. (1955) A behavioral model of rational choice. The Quarterly Journal of Economics 69:99-118. [aEMP]

Simon, H. A. (1956) Rational choice and the structure of the environment. Psychological Review 63:129-38. [JNM]

Sloman, S. A. (1993) Feature-based induction. Cognitive Psychology 25:231-80. [aEMP]

Sloman, S. A. (1996) The empirical case for two systems of reasoning. Psychological Bulletin 119:3-22. [JNM]

Slovic, P. (1995) The construction of preferences. American Psychologist 50:36471. $[\mathrm{PK}]$

Smith, J. D., Ashby, F. G., Berg, M. E., Murphy, M. S., Spiering, B., Cook, R. G. \& Grace, R. C. (2011) Pigeons' categorization may be exclusively nonanalytic. Psychonomic Bulletin \& Review 18:422-28. [RCG]

Smith, J. D., Beran, M. J., Crossley, M. J., Boomer, J. \& Ashby, F. G. (2010) Implicit and explicit category learning by macaques (Macaca mulatta) and humans (Homo sapiens). Journal of Experimental Psychology: Animal Behavior Processes 36:54-65. [RCG]

Smith, J. D., Berg, M. E., Cook, R. G., Murphy, M. S., Crossley, M. J., Boomer, J., Spiering, B., Beran, M. J., Church, B. A., Ashby, F. G. \& Grace, R. C. (2012) Implicit and explicit categorization: A tale of four species. Neuroscience and Biobehavioral Reviews 36:2355-69. [RCG]

Smolensky, P. (1990) Tensor product variable binding and the representation of symbolic structures in connectionist networks. Artificial Intelligence 46:159216. [aEMP]

Smolensky, P., Goldrick, M. \& Mathis, D. (in press) Optimization and quantization in gradient symbol systems: a framework for integrating the continuous and the discrete in cognition. Cognitive Science. [rEMP]

Stapp, H. P. (1975) Bell's theorem and world process. Nuovo Cimento B 29:27076. [END]

Stephens, D. W. \& Krebs, J. R. (1986) Foraging theory. Princeton University Press. [RCG]

Sternberg, S. (1969) The discovery of processing stages: Extensions of Donders' method. In: Attention and Performance II. Acta Psychologica, ed. W. G. Koster, 30:276-315. [END]

Sternberg, S. (2011) Modular processes in mind and brain. Cognitive Neuropsychology 28(3-4):156-208. [AB] 
References/Pothos \& Busemeyer: Can quantum probability provide a new direction for cognitive modeling?

Stewart, T. C., Bekolay T. \& Eliasmith C. (2011) Neural representations of compositional structures: Representing and manipulating vector spaces with spiking neurons. Connection Science 22(3):145-53. [TCS]

Stewart, T. C., Choo F.-X. \& Eliasmith, C. (2010) Dynamic behaviour of a spiking model of action selection in the basal ganglia. In: Proceedings of the 10th International Conference on Cognitive Modeling, ed. D. D. Salvucci \& G. Gunzelmann, pp. 235-40. Drexel University. [TCS]

Stewart, T. C. \& Eliasmith C. (2011) Neural cognitive modelling: A biologically constrained spiking neuron model of the Tower of Hanoi task. In: Proceedings of the 33rd Annual Conference of the Cognitive Science Society, ed. L. Carlson, C. Hölscher \& T.F. Shipley, pp. 656-61. Cognitive Science Society. [TCS]

Stojanovic, I. (2012) Situation semantics. In: Identity, language, and mind: An introduction to the philosophy of John Perry, ed. A Newen \& R. Van Riel. CSLI Publications. [MO]

Stolarz-Fantino, S., Fantino, E., Zizzo, D. J. \&Wen, J. (2003) The conjunction effect: New evidence for robustness. American Journal of Psychology 116(1):15-34. [aEMP]

Storms, G., De Boeck, P., van Mechelen, I. \& Ruts, W. (2005) Not guppies, nor goldfish, but tumble dryers, Noriega, Jesse Jackson, panties, car crashes, bird books, and Stevie Wonder. Memory \& Cognition 26:143-45. [JAH]

Streater, R. F. (2000) Classical and quantum probability. Journal of Mathematical and Physics 41:3556-603. [AIH]

Tegmark, M. (2000) The importance of quantum decoherence in brain processes. Physical Review E 61:4194-206. [SRH, DM]

Tenenbaum, J. B. \& Griffiths, T. L. (2001) The rational basis of representativeness. In: Proceedings of the 23rd Annual Conference of the Cognitive Science Society, pp. 1036-41. [aEMP]

Tenenbaum, J. B., Kemp, C., Griffiths, T. L. \& Goodman, N. (2011) How to grow a mind: Statistics, structure, and abstraction. Science 331:1279-85. [aEMP]

Tentori, K., Bonini, N. \& Osherson, D. (2004) The conjunction fallacy: A misunderstanding about conjunction? Cognitive Science 28:467-77. [KT]

Tentori, K. \& Crupi, V. (2012) On the conjunction fallacy and the meaning of and, yet again: A reply to Hertwig, Benz, and Krauss (2008). Cognition 122:123-34. [aEMP]

Tentori, K., Crupi, V. \& Russo, S. (2013) On the determinants of the conjunction fallacy: Confirmation versus probability. Journal of Experimental Psychology: General 142: 235-55. [KT]

Todd, P. M., Gigerenzer, G. \& the ABC Research Group (2012) Ecological rationality: Intelligence in the world. Oxford University Press. [JNM]

Todd, R. J. (1999) Back to our beginnings: Information utilization, Bertram Brookes and the fundamental equation of information science. Information Processing and Management 35(6):851-70. [AC]

Tononi, G., Sporns, O. \& Edelman, G. M. (1999) Measures of degeneracy and redundancy in biological networks. Proceedings of the National Academy of Sciences of the United States America 96(6):3257-62. [AB]

Tourangeau, R., Rips, L. J. \& Rasinski, K. A. (2000) The psychology of survey response. Cambridge University Press. [aEMP]

Townsend, J. T. (1984) Uncovering mental processes with factorial experiments. Journal of Mathematical Psychology 28:363-400. [END]

Townsend, J. T. \& Schweickert, R. (1989) Toward the trichotomy method of reaction times: Laying the foundation of stochastic mental networks. Journal of Mathematical Psychology 33:309-27. [END]

Townsend, J. T., Silva, K. M., Spencer-Smith, J. \& Wenger, M. (2000) Exploring the relations between categorization and decision making with regard to realistic face stimuli. Pragmatics and Cognition 8:83-105. [aEMP]

Treisman, M. (2002) Is signal detection theory fundamentally flawed? A response to Balakrishnan (1998a, Balakrishnan1998b, 1999). Psychonomic Bulletin d Review 9:845-57. [MVCB]

Trueblood, J. S. \& Busemeyer, J. R. (2011) A comparison of the belief-adjustment model and the quantum inference model as explanations of order effects in human inference. Cognitive Science 35(8):1518-52. [RB, arEMP]

Tversky, A. (1972) Elimination by aspects: A theory of choice. Psychological Review 79:281-99. [TR]

Tversky, A. (1977) Features of similarity. Psychological Review 84(4):327-52. $[\mathrm{TJP}, \mathrm{aEMP}]$

Tversky, A. \& Kahneman, D. (1973) Availability: A heuristic for judging frequency and probability. Cognitive Psychology 5:207-32. [aEMP]
Tversky, A. \& Kahneman, D. (1974) Judgment under uncertainty: Heuristics and biases. Science 185:1124-31. [aEMP]

Tversky, A. \& Kahneman, D. (1980) Causal schemata in judgments under uncertainty. In: Progress in social psychology, Vol. 1, ed. M. Fishbein, pp. 49-72. Erlbaum. [RCG]

Tversky, A. \& Kahneman, D. (1982) Judgments of and by representativeness. In: Judgment under uncertainty: Heuristics and biases, ed. D. Kahneman, P. Slovic \& A. Tversky, pp. 84-98). Cambridge University Press. [KT]

Tversky, A. \& Kahneman, D. (1983) Extensional versus intuitive reasoning: The conjunction fallacy in probability judgment. Psychological Review 90(4): 293-315. [JAH, PK, TJP, arEMP]

Tversky, A. \& Kahneman, D. (1992) Advances in prospect theory: Cumulative representation of uncertainty. Journal of Risk and Uncertainty 5:297-323. $[\mathrm{PK}]$

Tversky, A. \& Kahneman, D. (2004) Extensional versus intuitive reasoning: The conjunction fallacy in probability judgment. In: Preference, belief, and similarity: Selected writings by Amos Tversky, ed. E. Shafir, pp. 221-56. MIT Press. [CB]

Tversky, A. \& Koehler, D. J. (1994) Support theory: A nonextensional representation of subjective probability. Psychological Review 101:547-67. [PK, aEMP]

Tversky, A. \& Shafir, E. (1992) The disjunction effect in choice under uncertainty. Psychological Science 3:305-309. [PK, aEMP]

van Benthem, J. (2011) Logical dynamics of information and interaction. Cambridge University Press. [RB]

van Rijsbergen, C. (2004) The geometry of information retrieval. Cambridge University Press. [CB]

von Neumann, J. \& Morgenstern, O. (1947) Theory of games and economic behavior (2nd ed.). Princeton University Press. [PK]

Vitiello, G. (1995) Dissipation and memory capacity in the quantum brain model. International Journal of Modern Physics B9:973-89. [AC, aEMP]

Volz, K. G., Schooler, L. J. \& von Cramon, D. Y. (2010) It just felt right: The neural correlates of the fluency heuristic. Consciousness and Cognition 19:829-37. [JNM]

von Neumann, J. \& Morgenstern, O. (1947) Theory of games and economic behavior (2nd ed.). Princeton University. [PK]

Wakker, P. P. (2010) Prospect theory for risk and ambiguity. Cambridge University Press. [aEMP]

Walker, L., Thibaut, J. \& Andreoli, V. (1972) Order of presentation at trial. Yale Law Journal 82:216-26. [aEMP]

Wang, Z. \& Busemeyer, J. R. (in press) A quantum question order model supported by empirical tests of an a priori and precise prediction. Topics in Cognitive Science. [arEMP]

Wang, Z. J., Busemeyer, J. R., Atmanspacher, H. \& Pothos, E. M. (in press) The potential for using quantum theory to build models of cognition. Topics in Cognitive Science. [aEMP]

Wason, P. C. (1960) On the failure to eliminate hypotheses in a conceptual task. Quarterly Journal of Experimental Psychology 12:129-40. [aEMP]

Wedell, D. H. \& Moro, R. (2008) Testing boundary conditions for the conjunction fallacy: Effects of response mode, conceptual focus, and problem type. Cognition 107:105-36. [aEMP]

Weiss, D. J., Edwards, W. \& Weiss, J. W. (2009) The clinical significance decision. In: A science of decision making: The legacy of Ward Edwards, ed. J. W. Weiss \& D. J. Weiss, pp. 256-61. Oxford University Press. [JS]

Werner, R. F. \& Wolf, M. M. (2001a) All multipartite Bell correlation inequalities for two dichotomic observables per site. arXiv:quant-ph/0102024. [END]

Werner, R. F. \& Wolf, M. M. (2001b) Bell inequalities and entanglement. arXiv: quant-ph/0107093. [END]

Widdows, D. (2004) Geometry and meaning. CSLI Publications. [CB]

Wills, A. J. \& Pothos, E. M. (2012) On the adequacy of current empirical evaluations of formal models of categorization. Psychological Bulletin 138:102-25. [aEMP]

Young, F. W. (1996, June) New directions in psychometrics. Paper presented at the meeting of the Psychometric Society, Banff, -Canada. [JS]

Yukalov, V. \& Sornette, D. (2010) Decision theory with prospect interference and entanglement. Theory and Decision 70:283-328. [aEMP] 